\title{
Chemotherapy-induced peripheral neuropathy
}

Citation for published version (APA):

Peters - Beijers, T. (2016). Chemotherapy-induced peripheral neuropathy: an underestimated side effect with major impact on quality of life. [Doctoral Thesis, Maastricht University]. Ipskamp drukkers. https://doi.org/10.26481/dis.20161213tp

Document status and date:

Published: 01/01/2016

DOI:

10.26481/dis.20161213tp

Document Version:

Publisher's PDF, also known as Version of record

\section{Please check the document version of this publication:}

- A submitted manuscript is the version of the article upon submission and before peer-review. There can be important differences between the submitted version and the official published version of record.

People interested in the research are advised to contact the author for the final version of the publication, or visit the DOI to the publisher's website.

- The final author version and the galley proof are versions of the publication after peer review.

- The final published version features the final layout of the paper including the volume, issue and page numbers.

Link to publication

\footnotetext{
General rights rights.

- You may freely distribute the URL identifying the publication in the public portal. please follow below link for the End User Agreement:

www.umlib.nl/taverne-license

Take down policy

If you believe that this document breaches copyright please contact us at:

repository@maastrichtuniversity.nl

providing details and we will investigate your claim.
}

Copyright and moral rights for the publications made accessible in the public portal are retained by the authors and/or other copyright owners and it is a condition of accessing publications that users recognise and abide by the legal requirements associated with these

- Users may download and print one copy of any publication from the public portal for the purpose of private study or research.

- You may not further distribute the material or use it for any profit-making activity or commercial gain

If the publication is distributed under the terms of Article $25 \mathrm{fa}$ of the Dutch Copyright Act, indicated by the "Taverne" license above, 


\section{Chemotherapy-induced peripheral neuropathy:}

An underestimated side effect with major impact on quality of life 
Copyright (c) 2016 by A.J.M. Beijers.

All rights reserved. No parts of this book may be reproduced in any form or by means without the written permission of the author, or when appropriate, by the publisher of the publications.

Cover illustrations: Hans Peters

Thesis lay out: Tiny Wouters

Printed by: Ipskamp Drukkers, Enschede

ISBN: $\quad$ 978-94-028-0386-0

Reproduction of this thesis was kindly and unconditionally supported by the Netherlands Comprehensive Cancer Organisation (Integraal Kankercentrum Nederland); Máxima Medical Center Academy; Máxima Center of Oncology, Department of Research, which is gratefully acknowledged. 


\section{Chemotherapy-induced peripheral neuropathy:}

\section{An underestimated side effect with major impact on quality of life}

\section{Proefschrift}

ter verkrijging van de graad van doctor aan de Universiteit Maastricht, op gezag van de Rector Magnificus, Prof dr. Rianne M. Letschert volgens het besluit van het College van Decanen,

in het openbaar te verdedigen op dinsdag 13 december om 16.00 uur

door

Antoinetta Josephina Maria Beijers

Geboren op 4 februari 1987 te Nijmegen 


\section{Promotores:}

Prof. dr. V.C.G. Tjan-Heijnen

Prof. dr. L.V. van de Poll-Franse, Tilburg University

\section{Copromotores:}

Dr. G. Vreugdenhil

Dr. F. Mols, Tilburg University

\section{Beoordelingscommissie:}

Prof. Dr. R.P. Koopmans (voorzitter)

Dr. J.L.M. Jongen, Erasmus MC Rotterdam

Dr. R.I. Lalisang

Prof. Dr. C.C.D. van der Rijt, Erasmus MC Rotterdam

Prof. Dr. H.C. Schouten 


\section{Contents}

$\begin{array}{lll}\text { Chapter } 1 & \text { General introduction and outline } & 7\end{array}$

Part I Chemotherapy-induced peripheral neuropathy and quality of life

Chapter 2 Chemotherapy-induced peripheral neuropathy and impact on

quality of life six months after treatment with taxanes and

platinum derivatives.

J Community Support Oncol. 2014 Nov;12(11):401-6.

Chapter 3 Chemotherapy-induced neuropathy and its association with

quality of life among 2- to 11-year colorectal cancer survivors:

Results from the population-based PROFILES registry.

J Clin Oncol. $2013 \mathrm{Jul} ; 31(21): 2699-707$.

Chapter 4 Chemotherapy-induced neuropathy in multiple myeloma:

influence on quality of life and development of a

questionnaire to compose Common Toxicity Criteria-grading

for use in daily clinical practice.

Support Care Cancer. 2016 Jun;24(6):2411-20.

Part II Chemotherapy-induced peripheral neuropathy and the influence of chemotherapy administration

Chapter 5 A systematic review on chronic oxaliplatin-induced peripheral

neuropathy and the relation with oxaliplatin administration.

Support Care Cancer. 2014 Jul;22(7):1999-2007.

Chapter 6 Peripheral neuropathy in colorectal cancer survivors:

The influence of oxaliplatin administration. Results from the population-based PROFILES registry.

Acta Oncol. 2015 Apr;54(4):463-9.

Chapter 7 The magnitude of neurotoxicity in patients with multiple myeloma and the impact of dose modifications: Results from the

population-based PROFILES study.

Submitted

Chapter 8 Summary and general discussion

Samenvatting (summary in Dutch)

Valorisation

Dankwoord (acknowledgements)

About the author 

Chapter 1

General introduction 
Chapter 1 


\section{General introduction}

In the Netherlands about 100,000 patients are confronted with the diagnosis of cancer each year. ${ }^{1}$ Besides surgery and radiotherapy, systemic therapy - amongst others chemotherapy - is an important cornerstone in the treatment of many cancers, which has led to improved survival rates. However, treatment with cytostatics may cause dose limiting side effects like nausea, trombocytopenia or neutropenia and peripheral neuropathy, which, as a result of dose modifications, delay and withdrawal, may lead to decreased chemotherapy efficacy and survival..$^{2-8}$ In addition, these side effects may affect patients' quality of life (QOL) during and long after the cessation of treatment.

Because of improved supportive treatment options for some side effects, such as chemotherapy-induced nausea and myelosuppression by the application of better anti-emetics and hematopoietic colony stimulating agents, chemotherapy-induced peripheral neuropathy (CIPN) more often becomes the major dose limiting side effect of commonly used chemotherapeutic agents. CIPN is a severe, unpredictable, and often irreversible adverse event that may affect patients daily functioning. Usually it presents as a tingling or prickling sensation, numbness, arching or burning pain and/or an unpleasant sense of touch in the toes and fingers which may spread to the legs and arms. ${ }^{3,9}$ In addition, muscle weakness and autonomic dysfunction may occur. ${ }^{3}$ Despite multiple studies, there is no consensus on the assessment, prevention and treatment of CIPN. ${ }^{10}$ Due to the growing prevalence of cancer and the increasing use of chemotherapeutic agents causing CIPN, more patients therefore are confronted with the short and long-term consequences of this adverse event, and consequently it is likely that CIPN will become a major survivorship issue. ${ }^{9}$

\section{Chemotherapy-induced peripheral neuropathy}

"It started with mild symptoms such as tingling and numbness in my hands and feet progressing to the feeling of wearing socks, even when I wasn't. When I dropped my first cup of coffee I thought I was being clumsy, but then I started dropping things regularly. It became hard to button a shirt and to do things with my hands that required precision. A couple of times I fell flat on my face, because I didn't know where my feet were. Often it felt like the soles of my feet were like rubber and rounded, causing me to lose balance. At night it felt like I was being stabbed with a knife with pain emanating from the bottom of my feet that would shoot up my legs." (Quote from a colorectal cancer (CRC) patient) 


\section{The peripheral nervous system and neurotoxic chemotherapeutic agents}

Chemotherapeutic agents aim to stop the cell division (mitosis) of rapidly dividing cells including malignant cells. This is achieved mainly by damaging DNA or cell mechanisms needed for the mitosis. However, due to the inability of chemotherapeutic agents to differentiate between malignant and healthy cells, side effects like nerve damage with CIPN may as a result occur. ${ }^{5}$ The peripheral nervous system, in contrast to the central nervous system, is particularly vulnerable to the neurotoxic damage of chemotherapy as it is not protected by the blood-nerve barrier. $^{3,11,12}$ The peripheral nervous system can be divided into the autonomic and the somatic nervous system that includes both the sensory (afferent) and motor (efferent) nerves. ${ }^{13,14}$ The peripheral nerves are formed by a gathering of neuronal cell bodies with their axons. Axons transmit afferent or efferent signals through electrochemical impulses from the site of the stimuli to the target cell. ${ }^{13}$ The axons can be divided into fast myelinated (A-fibres) or slow unmyelinated (C-fibres) fibres, wherein myeline, a fatty isolating layer, enables rapid electric impulse transmission. ${ }^{3}$ The motor nerves comprise large A-fibres subdivided into $\alpha, \beta$ and $\gamma$-fibres, whereas the sensory nerve fibres mainly consist out of small myelinated $A \delta$-fibres, and thinly unmyelinated C-fibres. ${ }^{13}$ (Figure 1.1).
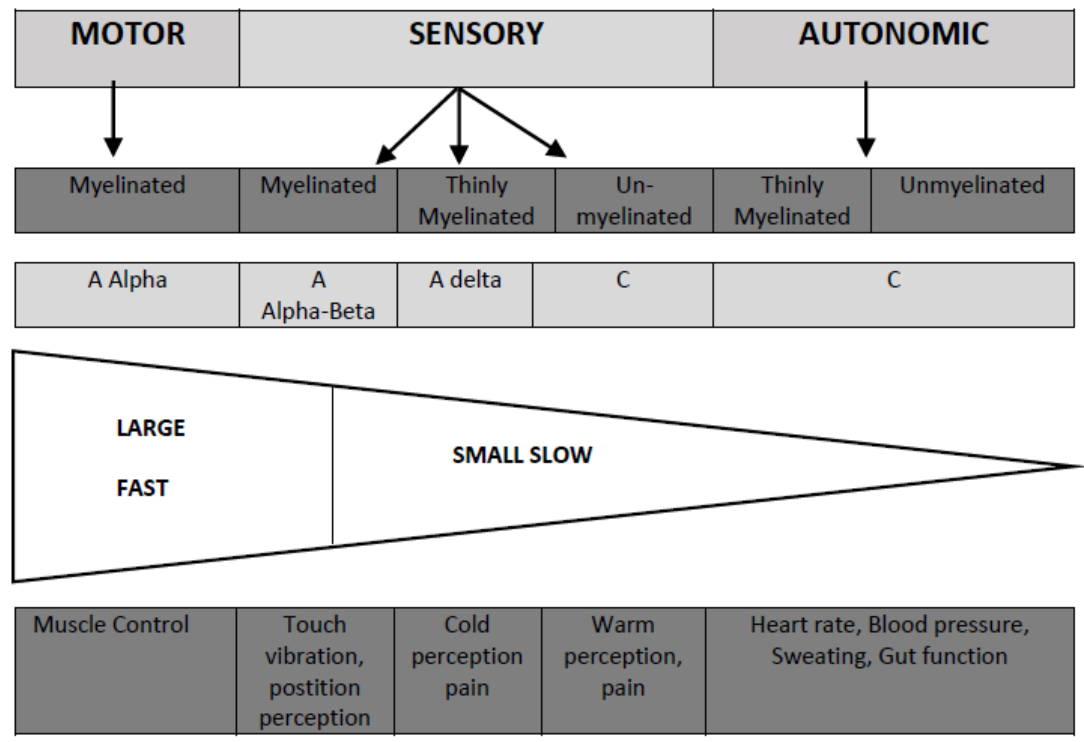

Figure 1.1 The peripheral nervous system. Adapted from Gutiérrez-Gutiérrez et al. ${ }^{3}$ 
Frequently used chemotherapeutic agents associated with CIPN include platinum compounds (oxaliplatin mainly used in the treatment of gastro-intestinal cancers e.g. CRC and cisplatin used in lung, ovary, bladder, head and neck, cervical and testicular cancer), taxanes (used in e.g. breast-, ovarian- and prostate cancer), vinca alkaloids (used in e.g. hematological malignancies), thalidomide, lenalidomide and the newer agents such as bortezomib (all used in hematological malignancies like multiple myeloma). ${ }^{3,47}$ The precise mechanism of CIPN remains unclear. However putative targets of these chemotherapeutic agents are the neuronal bodies (including mainly the dorsal root ganglion in sensory nerves), the myeline sheet or axonal components (including microtubules, ion channels or mitrochondria of the nerve fibres) ${ }^{11}$ (Figure 1.2).

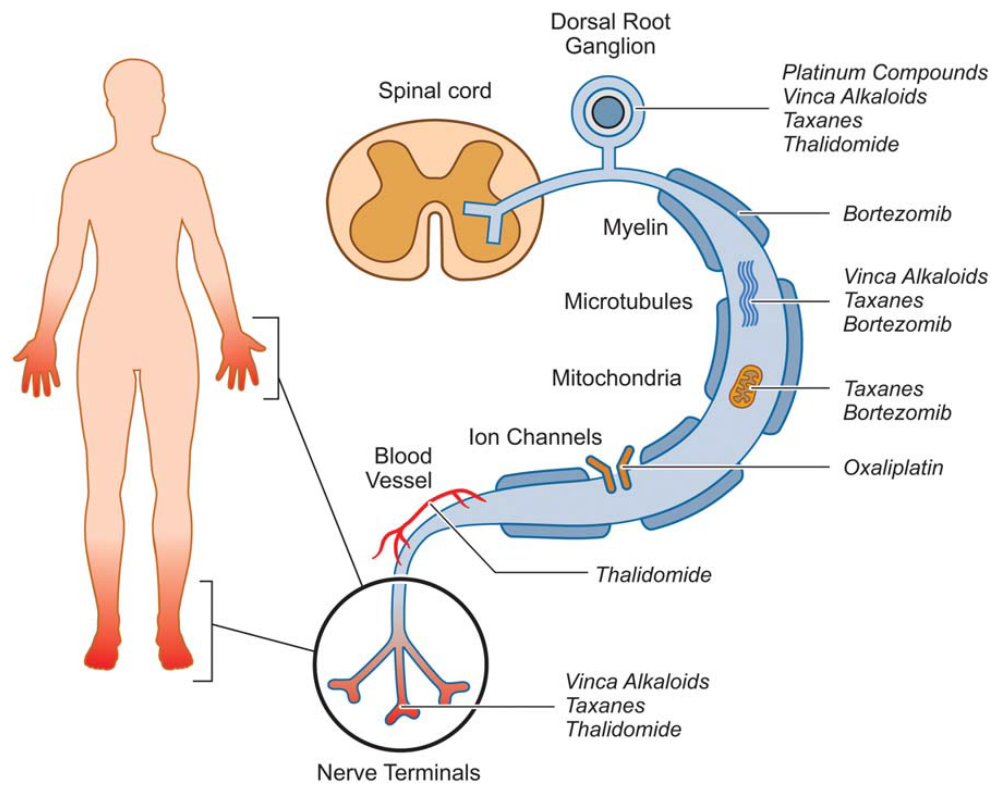

Figure 1.2 Stocking-and-glove distribution of CIPN toxicity with putative targets of the chemotherapeutic agents in the sensory nerve fibre; the neuronal cell body (dorsal root ganglion), myeline sheet or axonal components, including the microtubules, ion channels, mitochondria, and vascular network. Adapted from Park et al. ${ }^{12}$

\section{Prevalence and clinical manifestation of CIPN}

The incidence of CIPN varies between studies from 30 to $90 \%$ of patients receiving neurotoxic chemotherapy, including platinum compounds, taxanes, vinca alkaloids, 
immune-modulatory drugs and bortezomib..$^{2-4,6-8}$ The incidence and severity of CIPN is known to be dependent on the used chemotherapeutic agent, pre-existing neuropathy, cumulative dose, dose intensity and duration of exposure of the chemotherapeutic drug. ${ }^{4,7,8}$

The symptoms are predominantly sensory. ${ }^{8,11}$ In addition, the damage is mainly axonal and length-dependent resulting in a peripheral neuropathy with a symmetrical 'stocking-and-glove' distribution in the legs and arms. Symptoms present as paresthesias (tingling or prickling sensation), numbness, arching or burning pain and/or an unpleasant sense of touch in the toes and fingers. ${ }^{3,9}$ Impaired fine motor skills, e.g. buttoning a blouse, holding a pen or managing money are therefore likely to occur due to the sensory neuropathy. Motor neuropathy manifests as weakness, with difficulties in opening a bottle, walking stairs or standing, muscle wasting or cramps. ${ }^{3}$ Occasionally, dysfunction of the autonomic nervous system with symptoms as orthostatic dysfunction, impotence or dizziness occur. As symptoms of CIPN are often only partly reversible or even irreversible, they can result in impaired physical functioning or disability. ${ }^{3,4,6-8}$ In Table 1.1 an overview of the clinical manifestations of CIPN according to different types of neurotoxic chemotherapeutic agents are presented.

\section{Diagnosis of CIPN}

Many measurements to assess CIPN have been considered. ${ }^{15}$ These different assessment tools comprise clinical grading scales, subjective patient-reported outcome (PRO) measurements and objective assessment tools, e.g. nerve conduction studies.

The most commonly used measurements to grade CIPN in clinical trials and daily practice include common toxicity scales as the National Institute Common Terminology Criteria ( $\mathrm{NCl}-\mathrm{CTC}$ ), Eastern Cooperative Oncology (ECOG), World Health Organization (WHO) and Ajani criteria. ${ }^{15,16}$ These grading scales incorporate the severity of the sensory, motor and autonomic symptoms and the influence of these symptoms on daily functioning. The grading scales range from 0 , meaning asymptomatic, to 4 or 5 which refers to disabling.

The most frequently used patient-reported CIPN measurements incorporate both the assessment of QOL as well as CIPN, divided in questions about sensory, motor and autonomic dysfunction, and include the European Organisation for Research and Treatment of Cancer (EORTC) Quality of Life Questionnaire(QLQ)-C30 in combination with the CIPN20 ${ }^{17}$ and the Functional Assessment of Cancer Therapy/Gynaecologic Oncology Group (GOG)-neurotoxicity (FACT/GOG-Ntx). ${ }^{18}$ 


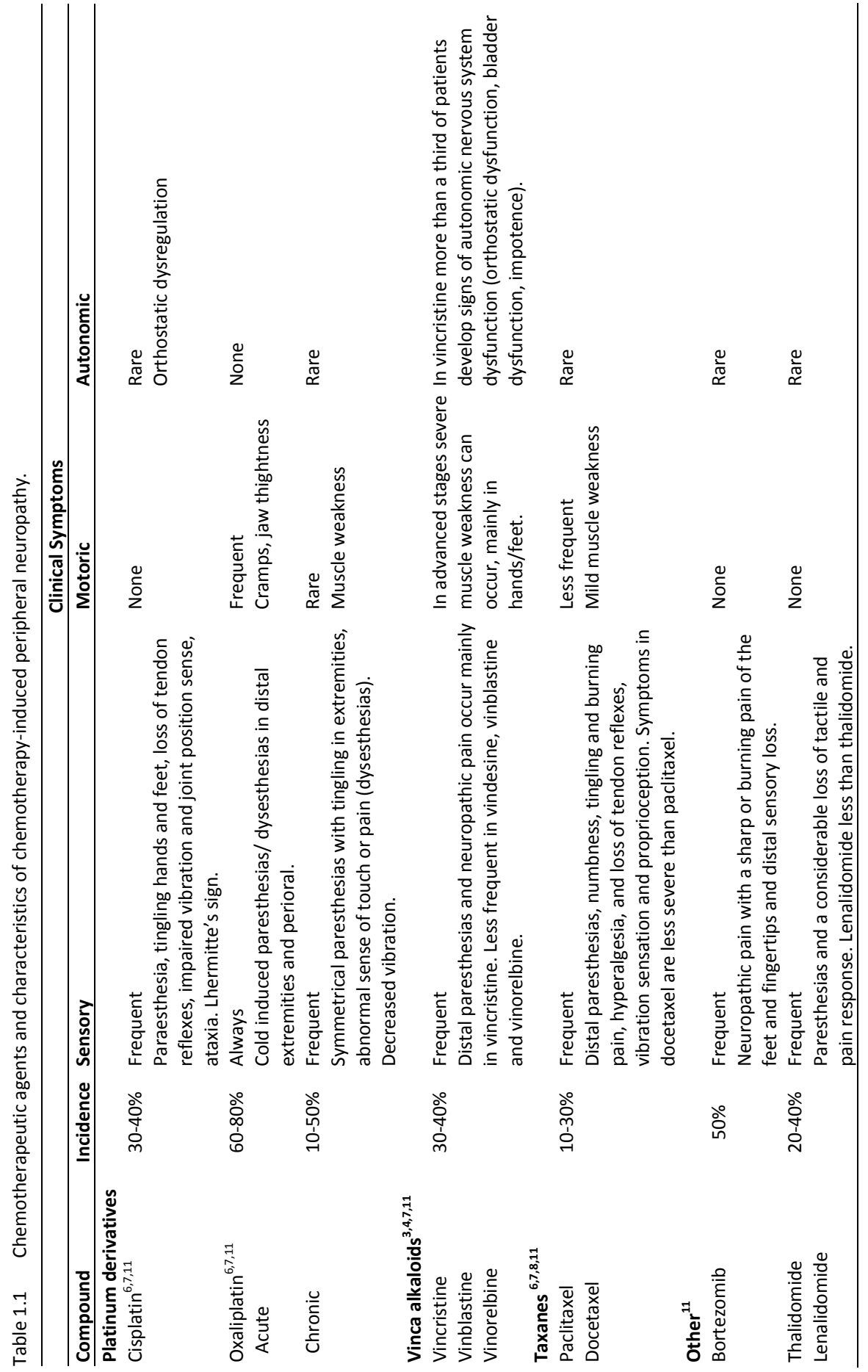


Furthermore, composite scales include both subjective and objective measurements (e.g. the Total Neuropathy Score (TNS)), and have mainly been developed by neurologists. ${ }^{19}$ Nevertheless, the currently available tools are not satisfactory for the assessment of CIPN in cancer patients, and are not developed for daily clinical practice. Therefore, it is of importance that a valid, reproducible and patient-friendly assessment tool, which can be used in daily practice, will be developed to measure CIPN.

\section{Prevention and treatment of CIPN}

Despite multiple studies there is still no consensus on how to prevent or treat CIPN. ${ }^{9}$ Several neuroprotective strategies, including $\mathrm{Ca} / \mathrm{Mg}$ infusion, amifostine, glutathione, glutamine, acetyl-L-carnitine and erythropoietin have been investigated. ${ }^{20-38}$ However, clinical evidence for standard use is insufficient and studies are described to be of low quality. ${ }^{9,39}$ Dutch guidelines on neuropathic pain in cancer patients recommend treatment with the opioid methadone, the antiepileptic agents gabapentin, pregabalin, or tricyclic antidepressants (TCAs). ${ }^{40}$ However, these recommendations are usually based on studies concerning "benign" neuropathic pain, such as postherpetic neuralgia, painful diabetic neuropathy, and trigeminal neuralgia instead of studies investigating the treatment of CIPN. ${ }^{41}$ These guidelines will be updated in the near future and an in advance performed Dutch review concerning pharmacological treatment of pain in cancer currently recommends treatment with duloxetine, gabapentin, or pregabalin for chemotherapy-induced neuropathic pain. ${ }^{42}$ Nevertheless, these recommendations were also acknowledged to be weak and based on low quality evidence. In addition, the recently developed ASCO guideline also did not strongly recommend any agent for the prevention or management of $\mathrm{CIPN}$. ${ }^{9}$ Only a moderate recommendation in favor of duloxetine was given. Therefore dose modification and alternative regimens remain necessary to limit the amount of damage associated with neurotoxic chemotherapy.

\section{CIPN and quality of life}

"The neuropathy has been quite underestimated by me. In particular, the neuropathy symptoms to the feet and legs, which provide a considerable violation on my quality of life six months after treatment. I think that more attention to this side effect is very important." (Quote from a 50 year old CRC patient) 
Since the progression free and overall survival of cancer is increasing, the side effects of treatment and QOL of cancer survivors are recognized to be more important. The World Health Organization (WHO) has defined QOL as "an individual's perception of their position in life in the context of the culture and value systems in which they live and in relation to their goals, expectations, standards and concerns" ${ }^{43}$ Symptoms of CIPN may have a negative impact on patients' individual goals, expectations and functioning and therefore are important for an individuals' QOL. For example, a piano teacher who has received chemotherapy for the treatment of CRC has numbness and tingling in the hands/fingers as a side effect of the treatment. Due to these symptoms she is not able to perform her occupation and hobby anymore. As expected this will influence her individual goals, expectations and functioning with a negative influence on her QOL as a consequence. In spite of this hypothesized major impact on patients' QOL little is known about the occurrence (short and/or long-term) of CIPN and the actual impact on patients' daily functioning. Due to the growing prevalence of cancer and the fact that treatment of CIPN remains challenging, more patients are confronted with the consequences of this adverse event. Therefore research concerning the influence of CIPN on patients' QOL is of major importance.

\section{Objectives of this thesis}

The main objectives of this thesis were to gain knowledge about the severity and prevalence of CIPN, the influence of CIPN on patients' quality of life (QOL) and the influence of chemotherapy administration on the development of CIPN. This thesis is subdivided into two parts. Within Table 1.2 an overview is given about current knowledge and the unresolved questions concerning CIPN and contents included within this thesis. Part one addresses the occurrence and severity of CIPN symptoms and the impact of those symptoms on the QOL among different cancer patients, at different time points after diagnosis. Part two addresses the influence of treatment with neurotoxic agents on the development of persistent CIPN, with a focus on oxaliplatin-induced neuropathy in CRC survivors and CIPN due to multiple myeloma (MM) treatment. 


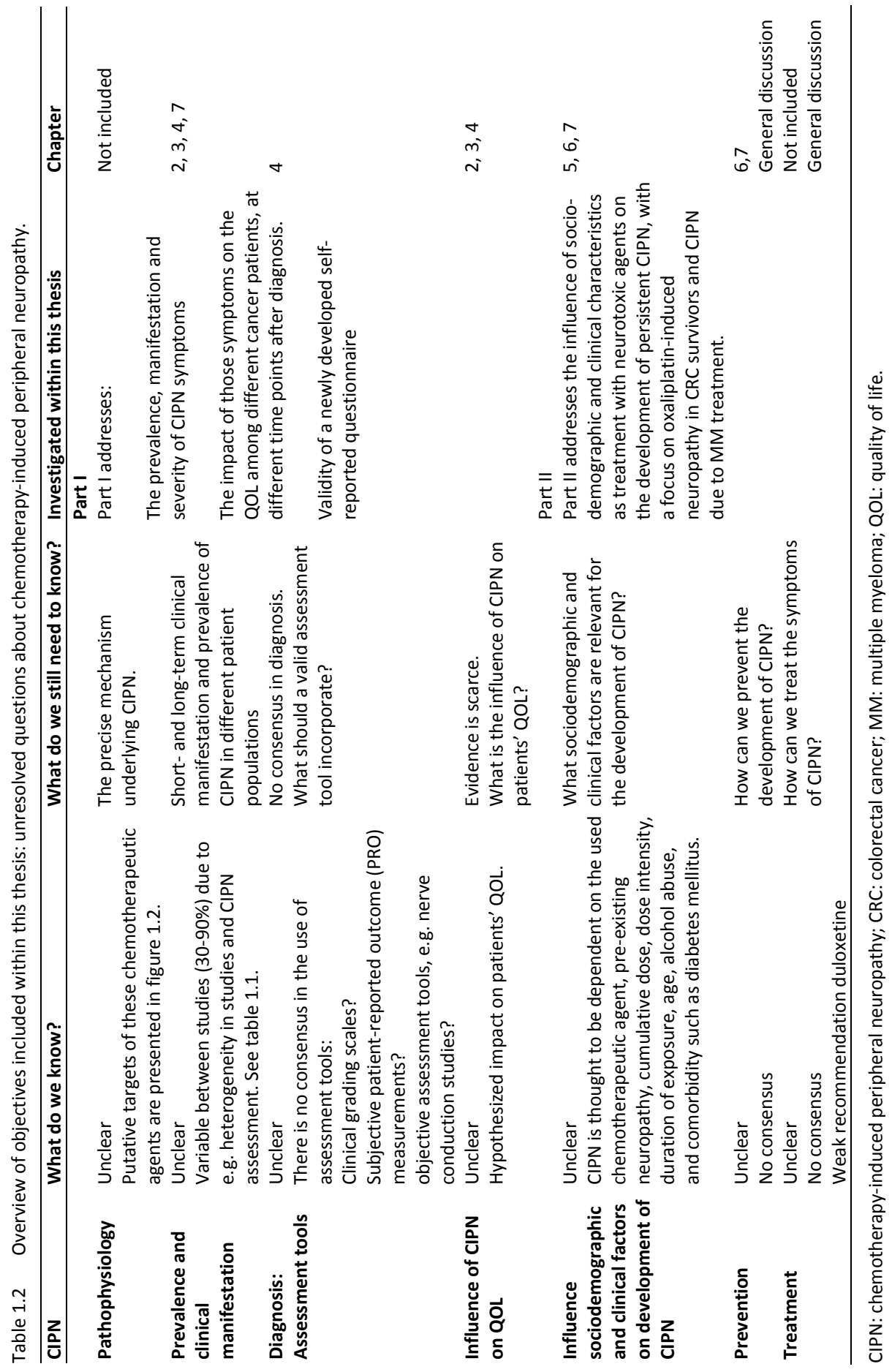




\section{Outline of this thesis}

\section{Part I - Chemotherapy-induced peripheral neuropathy and quality of life}

CIPN is a common dose limiting side effect of neurotoxic chemotherapeutics. Despite multiple studies, there is still no consensus on the prevention or treatment of CIPN and patients with remaining complaints of neuropathy after six months are at risk of having irreversible CIPN with difficulties in daily functioning. In chapter $\mathbf{2}$ we investigated the occurrence and severity of CIPN symptoms induced by platinum derivatives and taxanes and the impact of these symptoms on daily functioning and QOL in a sample of cancer patients visiting the outpatient clinical ward of the Máxima Medical Center median six months after cessation of treatment.

Oxaliplatin, first successfully used in the management of advanced CRC, is nowadays the regimen of choice for adjuvant treatment of patients with curatively resected node-positive CRC with improved survival rates as a consequence. Therefore the use of oxaliplatin has increased and more patients are living with its long-term side effects. In chapter 3 we investigated the long-term (2-11 years) prevalence of CIPN, and the burden on QOL in a population-based cohort of CRC survivors.

Another group of patients in whom CIPN is a common dose limiting side effect, are the more vulnerable patients with $\mathrm{MM}$. Those patients receive different neurotoxic agents (e.g. thalidomide, bortezomib, lenalidomide and vincristine) than CRC patients. Many assessment tools for monitoring CIPN symptoms have been investigated, however the currently available tools are not satisfactory in measuring CIPN, and are not developed for use in clinical practice. Therefore, in chapter 4 the validity of a newly developed self-reported neuropathy questionnaire, which has been developed for use in daily clinical practice, was performed. Also the prevalence and severity of CIPN due to these neurotoxic agents and the impact of CIPN on QOL in MM patients was investigated.

\section{Part II - CIPN and the influence of chemotherapy administration}

In the literature, the severity of persistent CIPN is described to be dependent on cumulative dose, duration of administration and dose intensity. Especially since there is no proven management for CIPN, currently dose modification or discontinuation are assumed to be the only way to minimize long-lasting CIPN. In addition, it is difficult to identify patients at risk of developing CIPN. In part II the influence of chemotherapy administration and dose modifications on the development of CIPN was investigated. The influence of oxaliplatin administration is mainly investigated in relation to the development of acute neurotoxicity during treatment instead of persistent CIPN. Therefore, in chapter $\mathbf{5}$ a systematic review of the literature that reports about the 
influence of oxaliplatin administration (e.g. cumulative dose, dose intensity, number of cycles and combination regimen) on the long-term prevalence and course of oxaliplatin-induced peripheral neuropathy was performed.

In chapter 6 we investigated the influence of oxaliplatin administration on the longterm severity and prevalence of CIPN among a population-based cohort of CRC survivors.

The association between sociodemographic and clinical characteristics, including treatment with thalidomide, lenalidomide, bortezomib and vincristine, and the prevalence and severity of CIPN in a population-based cohort of patients with MM is reported in chapter 7 .

Finally, in chapter $\mathbf{8}$ the main findings of this thesis are discussed and implications for future research and clinical practice are given.

\section{Setting and background}

To perform the studies included in this thesis, patient-reported outcomes and detailed clinical and treatment information was gathered from the included patients. In chapter 2 disease-specific CIPN measurements and treatment information was collected from cancer patients receiving different types of neurotoxic agents visiting the outpatient clinical ward from the Máxima Medical Center. Furthermore, clinical data from the population-based cohort of CRC (chapters 3 and 6) and patients with $\mathrm{MM}$ (chapters 4 and 7) registered within the Netherlands Cancer Registry (NCR), were collected from the NCR and medical files. Patient-reported outcomes in these studies were collected by means of the PROFILES registry. ${ }^{44}$ In addition, for the MM patients additional treatment data was obtained from the PHAROS registry.

The population-based studies were conducted in cooperation with the Netherlands Comprehensive Cancer Organisation (IKNL).

\section{Netherlands Cancer Registry, Southeastern region}

The Southeastern region of the Netherlands Cancer Registry, previously the Eindhoven Cancer Registry (ECR), started to collect data on all newly diagnosed cancer patients directly from pathology reports and medical records since 1955. Since 1989, the population-based ECR is part of the Netherlands Cancer Registry (NCR), and since 2008 data is collected in a national online database. The southeastern region of the NCR now comprises 2.4 million inhabitants, whom are referred to 10 general hospitals at 16 locations. The region is served by 6 regional pathology laboratories, and 2 large radiotherapy institutes. The NCR routinely collects sociodemographic characteristics 
of patients, including gender, date of birth, and clinical data like date of diagnosis, tumour stage, primary treatment and number of co morbidities.

\section{PROFILES}

The Patient Reported Outcomes/Quality of Life data collection in the populationbased studies (e.g. chapter 3, 4, 6 and 7), was done in PROFILES (Patient Reported Outcomes Following Initial Treatment and Long Term Evaluation of Survivorship), which is a registry for the study of the physical and psychosocial impact of cancer and its treatment from a growing population-based cohort of short and long-term cancer survivors. ${ }^{44}$ PROFILES is a tool that enables data collection management; from inviting patients to participation in studies, to collecting patient-reported outcome data via web-based or mailed questionnaires, and linking these data to clinical data from the cancer registry.

\section{PHAROS}

The Population based Hematology Registry for Observation Studies (PHAROS) is an extension of the Netherlands Cancer Registry in collaboration with the HOVON (Dutch Cooperative Group on Hemato-Oncology) and iMTA (institute for Medical Technology Assessment). It has the goal to provide insight into the current variance, quality and efficiency of hemato-oncological care in the Netherlands. PHAROS contains data about received treatments, side effects and treatment outcomes in more detail and therefore complements the data obtained from the NCR. PHAROS data is extracted from the medical files by trained NCR data managers. PHAROS data was used in chapter 4 and 7. 


\section{References}

1. http://www.cijfersoverkanker.nl/incidentie-sterfte-50.html (Accessed on 15 July, 2016).

2. Farquhar-Smith P. Chemotherapy-induced neuropathic pain. Curr Opin Support Palliat Care. 2011; 5(1):1-7.

3. Gutiérrez-Gutiérrez G, Sereno M, Miralles A, Casado-Sáenz E, Gutiérrez-Rivas E. Chemotherapyinduced peripheral neuropathy: clinical features, diagnosis, prevention and treatment strategies. Clin Transl Oncol. 2010;12(2):81-91.

4. Kannarkat G, Lasher E, Schiff D. Neurologic complications of chemotherapy agents. Curr Opin Neurol. 2007;20(6):719-725

5. Links $M$, Lewis C. Chemoprotectants: a review of their clinical pharmacology and therapeutic efficacy. Drugs. 1999;57(3):293-308.

6. Ocean A, Vahdat L. Chemotherapy-induced peripheral neuropathy: pathogenesis and emerging therapies. Support Care Cancer. 2004 Sep;12(9):619-625.

7. Quasthoff S, Hartung H. Chemotherapy-induced peripheral neuropathy. J Neurol. 2002;249(1):9-17.

8. Wolf S, Barton D, Kottschade L, Grothey A, Loprinzi C. Chemotherapy-induced peripheral neuropathy: prevention and treatment strategies. Eur J Cancer. 2008;44(11):1507-1515.

9. Hershman DL, Lacchetti C, Dworkin RH, Lavoie Smith EM, Bleeker J, Cavaletti G, et al. Prevention and management of chemotherapy-induced peripheral neuropathy in survivors of adult cancers: american society of clinical oncology clinical practice guideline. J Clin Oncol. 2014;32(18):1941-1967

10. Hershman DL, Lacchetti C, Dworkin RH, Lavoie Smith EM, Bleeker J, Cavaletti G, et al. Prevention and management of chemotherapy-induced peripheral neuropathy in survivors of adult cancers: American Society of Clinical Oncology clinical practice guideline. J Clin Oncol. 2014;32(18):1941-67.

11. Argyriou AA, Bruna J, Marmiroli P, Cavaletti G. Chemotherapy-induced peripheral neurotoxicity (CIPN): an update. Crit Rev Oncol Hematol. 2012;82(1):51-77.

12. Park SB, Goldstein D, Krishnan AV, Lin CS, Friedlander ML, Cassidy J, et al. Chemotherapy-induced peripheral neurotoxicity: A critical analysis. CA Cancer J Clin. 2013;63(6):419-437.

13. Guyton AC, Hall JE. Textbook of Medical Physiology. Pennsylvania: Elsevier Saunders; 2006.

14. Hijdra A, Koudstaal PJ, Roos RAC. Neurologie. Maarssen: Elsevier 2003.

15. Cavaletti G, Frigeni B, Lanzani F, Mattavelli L, Susani E, Alberti P, et al. Chemotherapy-Induced Peripheral Neurotoxicity assessment: a critical revision of the currently available tools. Eur J Cancer. 2010;46(3):479-494.

16. Griffith KA, Merkies IS, Hill EE, Cornblath DR. Measures of chemotherapy-induced peripheral neuropathy: a systematic review of psychometric properties. J Peripher Nerv Syst. 2010;1:314-325.

17. Postma TJ, Aaronson NK, Heimans JJ, Muller MJ, Hildebrand JG, Delattre JY, et al. The development of an EORTC quality of life questionnaire to assess chemotherapy-induced peripheral neuropathy: the QLQ-CIPN20. Eur J Cancer. 2005;41(8):1135-1139.

18. Huang HQ, Brady MF, Cella D, Fleming G. Validation and reduction of FACT/GOG-Ntx subscale for platinum/paclitaxel-induced neurologic symptoms: a gynecologic oncology group study. Int J Gynecol Cancer. 2007;17(2):387-393.

19. Smith EM, Beck SL, Cohen J. The total neuropathy score: a tool for measuring chemotherapy-induced peripheral neuropathy. Oncol Nurs Forum. 2008;35(1):96-102.

20. Bianchi R, Brines M, Lauria G, Savino C, Gilardini A, Nicolini G, et al. Protective effect of erythropoietin and its carbamylated derivative in experimental Cisplatin peripheral neurotoxicity. Clin Cancer Res. 2006;12(8):2607-2612.

21. Cervellini I, Bello E, Frapolli R, Porretta-Serapiglia C, Oggioni N, Canta A, et al. The neuroprotective effect of erythropoietin in docetaxel-induced peripheral neuropathy causes no reduction of antitumor activity in 13762 adenocarcinoma-bearing rats. Neurotox Res. 2010;18(2):151-160.

22. Kassem LA, Yassin NA. Role of erythropoeitin in prevention of chemotherapy-induced peripheral neuropathy. Pak J Biol Sci. 2010;13(12):577-587.

23. Keswani SC, Buldanlioglu U, Fischer A, Reed N, Polley $M$, Liang $H$, et al. A novel endogenous erythropoietin mediated pathway prevents axonal degeneration. Ann Neurol. 2004;56(6):815-826. 
24. Keswani SC, Leitz GJ, Hoke A. Erythropoietin is neuroprotective in models of HIV sensory neuropathy. Neurosci Lett. 2004;371(2-3):102-105.

25. Flatters SJ, Xiao WH, Bennett GJ. Acetyl-L-carnitine prevents and reduces paclitaxel-induced painful peripheral neuropathy. Neurosci Lett. 2006;397(3):219-223.

26. Bianchi G, Vitali G, Caraceni A, Ravaglia S, Capri G, Cundari S, et al. Symptomatic and neurophysiological responses of paclitaxel- or cisplatin-induced neuropathy to oral acetyl-L-carnitine. Eur J Cancer. 2005;41(12):1746-1750.

27. Ghirardi O, Lo Giudice P, Pisano C, Vertechy M, Bellucci A, Vesci L, et al. Acetyl-L-Carnitine prevents and reverts experimental chronic neurotoxicity induced by oxaliplatin, without altering its antitumor properties. Anticancer Res. 2005;25(4):2681-2687.

28. De Grandis D. Acetyl-L-carnitine for the treatment of chemotherapy-induced peripheral neuropathy: a short review. CNS Drugs. 2007; 21 Suppl 1:39-43; discussion 5-6.

29. Bianchi R, Gilardini A, Rodriguez-Menendez V, Oggioni N, Canta A, Colombo T, et al. Cisplatin-induced peripheral neuropathy: neuroprotection by erythropoietin without affecting tumour growth. Eur J Cancer. 2007;43(4):710-717.

30. Keswani SC, Bosch-Marce M, Reed N, Fischer A, Semenza GL, Hoke A. Nitric oxide prevents axonal degeneration by inducing HIF-1-dependent expression of erythropoietin. Proc Natl Acad Sci U S A. 2011;108(12):4986-4990.

31. Cascinu S, Catalano V, Cordella L, Labianca R, Giordani P, Baldelli AM, et al. Neuroprotective effect of reduced glutathione on oxaliplatin-based chemotherapy in advanced colorectal cancer: a randomized, double-blind, placebo-controlled trial. J Clin Oncol. 2002;20(16):3478-3483.

32. Milla P, Airoldi M, Weber G, Drescher A, Jaehde U, Cattel L. Administration of reduced glutathione in FOLFOX4 adjuvant treatment for colorectal cancer: effect on oxaliplatin pharmacokinetics, Pt-DNA adduct formation, and neurotoxicity. Anticancer Drugs. 2009;20(5):396-402.

33. Smyth JF, Bowman A, Perren T, Wilkinson P, Prescott RJ, Quinn KJ, et al. Glutathione reduces the toxicity and improves quality of life of women diagnosed with ovarian cancer treated with cisplatin: results of a double-blind, randomised trial. Ann Oncol. 1997;8(6):569-573.

34. De Vos FY, Bos AM, Schaapveld M, de Swart CA, de Graaf H, van der Zee AG, et al. A randomized phase II study of paclitaxel with carboplatin +/- amifostine as first line treatment in advanced ovarian carcinoma. Gynecol Oncol. 2005;97(1):60-67.

35. Hilpert F, Stahle A, Tome O, Burges A, Rossner D, Spathe K, et al. Neuroprotection with amifostine in the first-line treatment of advanced ovarian cancer with carboplatin/paclitaxel-based chemotherapy-a double-blind, placebo-controlled, randomized phase II study from the Arbeitsgemeinschaft Gynakologische Onkologoie (AGO) Ovarian Cancer Study Group. Support Care Cancer. 2005;13(10): 797-805.

36. Kemp G, Rose P, Lurain J, Berman M, Manetta A, Roullet B, et al. Amifostine pretreatment for protection against cyclophosphamide-induced and cisplatin-induced toxicities: results of a randomized control trial in patients with advanced ovarian cancer. J Clin Oncol. 1996;14:2101-2112.

37. Lorusso D, Ferrandina G, Greggi S, Gadducci A, Pignata S, Tateo S, et al. Phase III multicenter randomized trial of amifostine as cytoprotectant in first-line chemotherapy in ovarian cancer patients. Ann Oncol. 2003;14(7):1086-1093.

38. Openshaw H, Beamon K, Synold TW, Longmate J, Slatkin NE, Doroshow JH, et al. Neurophysiological study of peripheral neuropathy after high-dose Paclitaxel: lack of neuroprotective effect of amifostine. Clin Cancer Res. 2004;10(2):461-467.

39. A.J.M. Beijers, J.L.M. Jongen, G. Vreugdenhil. Chemotherapy-induced neurotoxicity: the value of neuroprotective strategies. Neth J Med. 2012;70(1):18-25.

40. Vissers KCP, Besse TC, Groot CM, Raats CJI, Rosenbrand CJGM, Vonk-Okhuijsen SY, et al. Landelijke richtlijnwerkgroep Pijn bij kanker 2008 01-10-2008; 1.1.

41. Finnerup NB, Sindrup SH, Jensen TS. The evidence for pharmacological treatment of neuropathic pain. Pain. 2010;150(3):573-581.

42. van den Beuken-van Everdingen MH, de Graeff A, Jongen JL, Dijkstra D, Mostovaya I, Vissers KC; national guideline working group "Diagnosis treatment of cancer pain". Pharmacological treatment of pain in cancer patients: The role of adjuvant analgesics, a systematic review. Pain Pract. 2016 May 21. 
43. The World Health Organization Quality of Life Assessment (WHOQOL): development and general psychometric properties. Soc Sci Med. 1998;46(12):1569-1585.

44. van de Poll-Franse LV, Horevoorts N, van Eenbergen M, Denollet J, Roukema JA, Aaronson NK, et al. The Patient Reported Outcomes Following Initial treatment and Long term Evaluation of Survivorship registry: scope, rationale and design of an infrastructure for the study of physical and psychosocial outcomes in cancer survivorship cohorts. Eur J Cancer. 2011;47(14):2188-94. 
Chemotherapy-induced peripheral neuropathy and quality of life 


\section{Chapter 2}

Chemotherapy-induced peripheral neuropathy and impact on quality of life 6 months after treatment with chemotherapy

Beijers A, Mols F, Dercksen W, Driessen C, Vreugdenhil G J Community Support Oncol 2014;12:401-406 


\title{
Abstract
}

\author{
Background \\ Chemotherapy-induced peripheral neuropathy (CIPN) is a major dose-limiting toxicity of \\ cytostatics. With improved survival among cancer patients, CIPN may have a major impact on \\ quality of life (QoL) of cancer survivors.
}

\section{Objective}

To determine the occurrence of CIPN induced by oxaliplatin and taxanes and its impact on QoL median 6 months after chemotherapy.

\section{Methods}

All patients who received their last treatment with oxaliplatin or taxanes in 2 consecutive years in the Máxima Medical Centre, the Netherlands, were eligible for the study. Neurotoxicity and its effect on QoL was assessed with the recently developed Chemotherapy Induced Neurotoxicity Questionnaire (CINQ) and the Functional Assessment of Cancer Therapy/Gynecologic Oncology Group-Neurotoxicity (FACT/GOG-Ntx) median 6 months after cessation of therapy.

\section{Results}

Of the 58 eligible patients, 43 (74.1\%) completed the questionnaire. After a median follow-up of 6.5 months after cessation of therapy, most of the patients experienced neurotoxicity in the upper and lower extremities $(78.8 \%$ and $89.7 \%$, respectively). Overall, the most-reported complaints included numbness and tingling in hands as well as feet, suffering from cold feet, and trouble distinguishing objects in the hands. Housekeeping difficulties were reported in $12.8 \%$ of patients, and $20.5 \%$ of patients became more dependent on others because of the neurotoxicity. Overall, QoL was negatively affected by the impact of CIPN in $48.6 \%$ of patients.

\section{Limitations}

Due to the small sample size selection bias cannot be ruled out and no data about CIPN during treatment were available.

\section{Conclusions}

After a median follow-up of 6.5 months after cessation of therapy with oxaliplatin or taxanes, CIPN is common and leads to impairment in patient QoL. More research is needed to assess the impact of neurotoxicity on QoL. 


\section{Introduction}

In cancer treatment, cytostatics are often accompanied by side-effects that may have a major impact on the quality of life (QoL) of patients. ${ }^{1}$ One of those side-effects is chemotherapy-induced peripheral neuropathy (CIPN), which is a major dose-limiting toxicity in the frequently used cytostatics, such as taxanes, to treat breast, ovarian, and prostate cancers, and the platinum derivative oxaliplatin, which are mainly used in colorectal cancer. ${ }^{2-5}$ CIPN symptoms consist mainly of sensory impairment, but may also affect motor functions and the autonomic nervous system. ${ }^{5-7}$ Patients with CIPN can experience symptoms in hands and feet, such as tingling, numbness, cramps, and aching or burning pain which can cause problems with regular daily activities such as buttoning clothes, holding a pen, opening a jar or bottle, or problems with standing, walking, or climbing stairs. The severity of these symptoms depends on the type of drug used, duration of administration, cumulative dose, and pre-existing peripheral neuropathy..$^{2-8}$ After 4-6 months, the neuropathy resolves in most patients, but it is most often irreversible in the patients (about $60 \%$ ) who still have complaints after 6 months. ${ }^{8,9}$ Despite multiple studies that have yielded promising results, there is still no consensus on how to prevent CIPN. $^{7}$ In addition, CIPN treatment also remains difficult and there is no well-accepted proven therapy. ${ }^{10,11}$ Because the use of chemotherapy is increasing in the treatment of cancer, CIPN is becoming a major survivorship issue that may have a negative impact on QoL.

Although many questionnaires have been developed to assess neuropathy and QoL, ${ }^{1,12-14}$ only a few studies have investigated the impact of CIPN on QoL as demonstrated by a recent systematic review. ${ }^{15}$ Accordingly, only 8 studies have reported that most patients had symptoms of CIPN and worse QoL because of CIPN, ${ }^{1,16-22}$ and 3 studies did not find a relation between CIPN and QoL. ${ }^{12,23,24}$ In addition, other studies have demonstrated the impact of CIPN on daily functioning, but did not assess QoL directly. ${ }^{25,26}$ The studies that directly assessed the influence of CIPN on QoL differed notably in applied study design, included patient population, and way of CIPN and QoL assessment, which makes comparison of these studies difficult. $^{1,12,16-18,20-22,24}$ In addition, CIPN and QoL assessments were completed over a range of follow-up periods, while patients with remaining complaints after 6 months are at risk of having irreversible CIPN. Therefore, recognition of CIPN and its influence on QoL is needed because of the importance of informing and guiding these patients. Consequently, more studies are required to investigate the direct impact of CIPN on QoL 6 months after therapy. Therefore, we aimed to study the occurrence of CIPN symptoms induced by oxaliplatin or taxanes and the impact of these symptoms on QoL median 6 months after cessation of treatment. 


\section{Methods}

\section{Patients}

Patients who received their last course with oxaliplatin or taxanes (paclitaxel or docetaxel) during 2 consecutive years in Máxima Medical Centre in the Netherlands were eligible for this study. The indications for treatment with chemotherapy were neoadjuvant, adjuvant, or palliative. Patients received a questionnaire median 6 months after their last chemotherapy course, and nonrespondents received 1 reminder telephone call. All of the respondents gave written informed consent. A local Medical Ethics Committee approved this study.

\section{Chemotherapy and cumulative dose}

Patients treated with oxaliplatin received a dose of $150-300 \mathrm{mg} / \mathrm{m}^{2}$ intravenously once in 3 weeks (cumulative doses ranging from $690-2,340 \mathrm{mg} / \mathrm{m}^{2}$ ). Paclitaxel was given in a dose of $80 \mathrm{mg} / \mathrm{m}^{2}$ weekly or $175 \mathrm{mg} / \mathrm{m}^{2}$ once in 3 weeks (cumulative doses ranging from $560-1,960 \mathrm{mg} / \mathrm{m}^{2}$ ). All patients who were treated with docetaxel received doses of $75 \mathrm{mg} / \mathrm{m}^{2}$ (cumulative doses ranging from $720-1,120 \mathrm{mg} / \mathrm{m}^{2}$ ). The median number of chemotherapy courses was 6 and the median duration of administration was 15 weeks. Dose reduction was applied in accordance to national guidelines.

\section{Measures}

Patients' sociodemographic and clinical characteristics, such as age, sex, type of malignancy and occurrence of metastatic disease, information on comorbid conditions and type and indication of chemotherapy, cumulative dose and duration of administration, were extracted from their medical files.

The recently developed Chemotherapy Induced Neurotoxicity Questionnaire (CINQ) ${ }^{1}$ and the validated Functional Assessment of Cancer Therapy/Gynaecologic Oncology Group-Neurotoxicity (FACT/GOG-Ntx) questionnaires were used to evaluate the symptoms of CIPN and the impact of CIPN on patients' QoL. ${ }^{27}$ The CINQ complements the FACT/GOG-Ntx because it asks patients about more and different symptoms and the relation of those symptoms to QoL. Patients were asked to answer the questions about symptoms they experienced in the previous 7 days.

The CINQ consists of common symptoms in the following the upper extremities, lower extremities, and head and neck regions; and it asks about other possible symptoms not already mentioned in the questionnaire. Symptom intensity is indicated on a Likert scale $(0=$ not at all to $5=$ very much). A higher score indicates more severe CIPN. The maximum achievable score was 520. The last question of the CINQ assesses the impact of CIPN on QoL on a scale of 1 (not at all) to 5 (very much). 
The FACT/GOG-Ntx has 2 parts: the FACT-GOG subscale comprised of 4 areas concerning QoL well-being - physical, social/familial, emotional, and functional; and the Ntx subscale containing 11 items that evaluate the amount of common neuropathy symptoms. Symptoms are indicated on a scale ranging from 0 (not at all) to 4 (very much). A higher score indicates more severe neuropathy. Maximum achievable score is 44 .

\section{Statistical analysis}

Sociodemographic and clinical characteristics of the included patients were shown in absolute numbers and percentages. The occurrence of neuropathy symptoms experienced in the previous week were shown in percentages and reflect the answers quite a bit, much, and very much of the CINQ combined, and somewhat, quite a bit, and very much combined for the FACT/GOG-Ntx. Furthermore, mean total scores on the Ntx-subscale and CINQ, and QoL questions were shown.

The impact of CIPN on QoL was assessed by a comparison of mean total scores of the FACT/GOG-Ntx and CINQ between the combined answers not at all, a little, and somewhat vs quite a bit or very much on the FACT-G item I am content with my QoL right now; and with the CINQ item How much do the complaints of hand, feet, and face reduce the QoL. Analysis of covariance (ANCOVA) were done with adjusted for indication of treatment and time of questionnaire. In addition, a comparison of mean FACT/GOG subscale scores were compared between low and high (eg, top $25 \%$ of patients) neuropathy symptom scores on FACT/GOG-Ntx with ANCOVA. Analyses were adjusted for indication of treatment, age, and time of questionnaire. The correlation between the CINQ and FACT/GOG-Ntx was computed with Pearson correlation. All $P$ values $<.05$ were considered to be significant. All analyses were conducted using SPSS version 19.0.

\section{Results}

\section{Demographics}

In all, 68 patients were considered eligible for our study. Ten patients died between the time of inclusion and sending of the questionnaire. Of the remaining 58 patients, 15 did not return the questionnaire despite having received a reminder telephone call. Thus, 43 patients completed the questionnaire (response rate, 74.1\%). The patients' sociodemographic and clinical characteristics are described in Table 2.1. 
Table 2.1 Sociodemographic and clinical characteristics of included patients.

\begin{tabular}{|c|c|}
\hline Patients & $\begin{array}{l}\text { Unless otherwise indicated } \\
\qquad \begin{array}{c}(\mathrm{N}=43) \\
\mathrm{n}(\%)\end{array}\end{array}$ \\
\hline \multicolumn{2}{|l|}{ Sex } \\
\hline Male & $17(40)$ \\
\hline Female & $26(61)$ \\
\hline Mean age, y (SD) & $60.7(11)$ \\
\hline \multicolumn{2}{|l|}{ Type malignancy } \\
\hline Colon carcinoma & $23(54)$ \\
\hline Rectal carcinoma & $5(12)$ \\
\hline Mamma carcinoma & $10(23)$ \\
\hline Prostate carcinoma & $1(2)$ \\
\hline Ovarian carcinoma & $3(7)$ \\
\hline Unknown & $1(2)$ \\
\hline \multicolumn{2}{|l|}{ Chemotherapy } \\
\hline Oxaliplatin & $29(67)$ \\
\hline Paclitaxel & $8(19)$ \\
\hline Docetaxel & $6(14)$ \\
\hline \multicolumn{2}{|l|}{ Metastatic disease } \\
\hline Yes & $23(54)$ \\
\hline No & $18(42)$ \\
\hline \multicolumn{2}{|l|}{ Indication } \\
\hline Neoadjuvant & $3(7)$ \\
\hline Adjuvant & $18(42)$ \\
\hline Palliative & $21(49)$ \\
\hline Unknown & $1(2)$ \\
\hline \multicolumn{2}{|l|}{ Mean cumulative dose, $\mathrm{mg} / \mathrm{m}^{2}$ (SD) } \\
\hline Oxaliplatin & $1,379(452)$ \\
\hline Paclitaxel & $1,120(508)$ \\
\hline Docetaxel & $893(205)$ \\
\hline Median no. of chemotherapy courses (range) & $6.0(3-10)$ \\
\hline Median duration of administration, weeks (range) & $15.5(3-27)$ \\
\hline Median months follow-up (range) & $6.5(3-14)$ \\
\hline Previous chemotherapy & $6(14)$ \\
\hline \multicolumn{2}{|l|}{ Comorbidities } \\
\hline Diabetes mellitus & $4(9)$ \\
\hline Multiple sclerosis & 0 \\
\hline Pre-existing neuropathy & 0 \\
\hline Hyperthyroidism & 0 \\
\hline Cerebrovascular accident & $2(5)$ \\
\hline Rheumatoid arthritis & 0 \\
\hline
\end{tabular}

${ }^{a}$ Percentages can exceed 100 because of rounding.

\section{Neuropathy}

After a median follow-up of 6.5 months after the cessation of therapy most of the patients had symptoms of CIPN in upper and lower extremities $(78.8 \%$ and $89.7 \%$, respectively). Overall, according to the FACT/GOG-Ntx-subscale, the symptoms that 
were reported by the patients during the previous week were mainly sensory and consisted of numbness or tingling $(63.3 \%$ and $60.9 \%)$ and discomfort of hands and feet (48.8\% and 56.1\%; Table 2.2). Table 2.3 shows the most frequently reported neuropathy symptoms, divided into sensory, motor, or autonomic symptoms according to the CINQ. Answers of the CINQ corresponded well to answers of the Ntx, and the most frequently reported complaints according to the CINQ included numbness in the fingers or feet $(48.7 \%$ and $51.2 \%)$, suffering from cold feet $(51.2 \%)$, tingling fingers or feet (39.5\% and $46.9 \%)$, trouble distinguishing objects in the hands (39.5\%), and loss of strength in the legs (38.6\%). Patients were limited in daily activities with major limitations being unbuttoning a blouse (39.5\%), picking up things from the floor $(27.0 \%)$, and opening a jar or bottle due to loss of strength in the hands (27.0\%). In addition, $12.8 \%$ of patients reported housekeeping difficulties, and $20.5 \%$ of patients stated that they became more dependent on others because of the CIPN. Mean total score on the Ntx-subscale was 13.4 (SD, 8.7; range 0-32) and the mean total score for the CINQ was 82.6 (SD, 64.5; range 1-230). In addition, total scores on the Ntx-subscale were significantly correlated to the total scores of the CINQ $(r=0.8$; $\mathrm{p}<.001)$.

Table 2.2 Patient responses to the most often reported neuropathy symptoms according to the FACT/GOG-Ntx $(n=41)$.

\begin{tabular}{|c|c|c|c|c|c|}
\hline Sensory symptom or problem & $\begin{array}{c}\text { Not at all } \\
\mathrm{n}(\%)\end{array}$ & $\begin{array}{c}\text { A little } \\
\text { n (\%) }\end{array}$ & $\begin{array}{c}\text { Somewhat } \\
n(\%)\end{array}$ & $\begin{array}{c}\text { Quite a bit } \\
\text { n (\%) }\end{array}$ & $\begin{array}{c}\text { Very much } \\
\mathrm{n}(\%)\end{array}$ \\
\hline Numbness or tingling in hands? & $10(24.4)$ & $8(19.5)$ & $6(14.6)$ & $14(34.1)$ & $6(14.6)$ \\
\hline Numbness or tingling in feet? & $11(26.8)$ & $5(12.2)$ & $5(12.2)$ & $14(34.1)$ & $6(14.6)$ \\
\hline Feeling of discomfort in hands? & $16(39.0)$ & $5(12.2)$ & $7(17.1)$ & $12(29.3)$ & $1(2.4)$ \\
\hline Feeling of discomfort in feet? & $14(34.1)$ & $4(9.8)$ & $5(12.2)$ & $13(31.7)$ & $5(12.2)$ \\
\hline Trouble buttoning a blouse? & $16(39.0)$ & $8(19.5)$ & $6(14.6)$ & $9(22.0)$ & $2(4.9)$ \\
\hline Trouble distinguishing objects in the hands & $20(48.8)$ & $4(9.8)$ & $7(17.1)$ & $8(19.5)$ & $2(4.9)$ \\
\hline
\end{tabular}

FACT/GOG-Ntx, Functional Assessment of Cancer therapy/ Gynaecologic Oncology Group-Neurotoxicity

\section{Impact neuropathy on quality of life}

On the FACT-G item I am content with my QoL right now, $48.8 \%$ of patients report to be quite a bit or very much content with their QoL. Patients who were less content with their QoL had worse total neuropathy scores than patients who were content with their QoL, as assessed with Ntx (15.7 vs. 11.0, respectively; $\mathrm{p}=.034$ ) and CINQ (93.8 vs. 70.0; $p=.267$ ). The last question of the CINQ How much do the complaints of hand, feet, and face reduce the QoL? showed that in $48.6 \%$ of patients (mean 1.86; SD, 1.1), QoL was negatively influenced because of CIPN (answers a little to very much combined). A significant correlation ( $r=0.37 ; p=.02)$ was found between scores on emotional well-being (FACT-G; range, 0-24; mean, 6.9; SD, 3.6) and neuropathy symptoms (Ntx-subscale; range, 0-32; mean, 13.4; SD, 8.7). Patients who reported 
many neuropathy symptoms on the FACT/GOG-Ntx (eg, top $25 \%$ of patients with highest scores) reported significantly worse emotional well-being (9.4 vs. 5.8; $p=.002$ ) than the patients with less neuropathy symptoms (eg, lower 75\%; Table 2.4). Other FACT-G subscales did not differ between the patients with lower and upper neuropathy scores.

Table 2.3 Patient responses to the most frequently reported neuropathy symptoms. ${ }^{\text {ab }}$

\begin{tabular}{|c|c|c|c|c|c|c|}
\hline Symptom or problem & $\begin{array}{l}\text { Not at all } \\
\mathrm{n}(\%)\end{array}$ & $\begin{array}{l}\text { Barely } \\
\mathrm{n}(\%)\end{array}$ & $\begin{array}{l}\text { A little } \\
\text { n (\%) }\end{array}$ & $\begin{array}{l}\text { Quite a bit } \\
\text { n (\%) }\end{array}$ & $\begin{array}{l}\text { Much } \\
\mathrm{n}(\%)\end{array}$ & $\begin{array}{l}\text { Very much } \\
\text { n (\%) }\end{array}$ \\
\hline Corresponding score & 0 & 1 & 2 & 3 & 4 & 5 \\
\hline \multicolumn{7}{|l|}{ Sensory } \\
\hline Tingling fingers or hands & $14(36.8)$ & $2(5.3)$ & $7(18.4)$ & $9(23.7)$ & $6(15.8)$ & 0 \\
\hline Tingling toes or feet & $15(38.5)$ & $1(2.6)$ & $4(10.3)$ & $8(20.5)$ & $9(23.1)$ & $2(5.1)$ \\
\hline Numbness fingers & $14(36.8)$ & $1(2.6)$ & $9(23.7)$ & $5(13.2)$ & $9(23.7)$ & 0 \\
\hline Numbness toes or feet & $16(40.1)$ & 0 & $3(7.7)$ & 7 (17.9) & $11(28.2)$ & $2(5.1)$ \\
\hline $\begin{array}{l}\text { Aching/burning pain in } \\
\text { fingers/hands with coldness }\end{array}$ & $21(55.3)$ & $3(7.9)$ & $1(2.6)$ & $7(18.4)$ & $3(7.9)$ & $3(7.9)$ \\
\hline $\begin{array}{l}\text { Aching/burning pain in } \\
\text { toes/feet }\end{array}$ & $28(71.8)$ & 0 & $2(5.1)$ & $4(10.3)$ & $5(12.8)$ & 0 \\
\hline $\begin{array}{l}\text { Trouble distinguishing } \\
\text { objects in hands }\end{array}$ & $17(44.7)$ & $2(5.3)$ & $4(10.5)$ & $5(13.2)$ & $4(10.5)$ & $6(15.8)$ \\
\hline Suffering from cold feet & $16(41.0)$ & $1(2.6)$ & $2(5.1)$ & $10(25.6)$ & $5(12.8)$ & $5(12.8)$ \\
\hline \multicolumn{7}{|l|}{ Motor } \\
\hline $\begin{array}{l}\text { Trouble unbuttoning a } \\
\text { blouse? }\end{array}$ & $15(40.5)$ & $2(5.4)$ & $9(24.3)$ & $5(13.5)$ & $3(8.1)$ & $3(8.1)$ \\
\hline $\begin{array}{l}\text { Trouble opening a jar or } \\
\text { bottle due to loss of } \\
\text { strength in hands? }\end{array}$ & $14(37.8)$ & $3(8.1)$ & $10(27.0)$ & $4(10.8)$ & $4(10.8)$ & $2(5.4)$ \\
\hline $\begin{array}{l}\text { Difficulty picking up little } \\
\text { things? }\end{array}$ & $15(40.5)$ & $4(10.8)$ & $8(21.6)$ & $4(10.8)$ & $3(8.1)$ & $3(8.1)$ \\
\hline $\begin{array}{l}\text { Difficulty pushing tablets } \\
\text { out of a strip? }\end{array}$ & $23(62.2)$ & $1(2.7)$ & $3(8.1)$ & $3(8.1)$ & $5(13.5)$ & $2(5.4)$ \\
\hline Less strength in legs? & $22(56.4)$ & $1(2.6)$ & $1(2.6)$ & $8(20.5)$ & $6(15.4)$ & $1(2.6)$ \\
\hline \multicolumn{7}{|l|}{ Autonomic } \\
\hline $\begin{array}{l}\text { Trouble with bladder } \\
\text { control? }\end{array}$ & $24(61.5)$ & $2(5.1)$ & $4(10.3)$ & $6(15.4)$ & $2(5.1)$ & $1(2.6)$ \\
\hline $\begin{array}{l}\text { Erectile difficulties } \\
\text { (only for men, } n=15 \text { ) }\end{array}$ & $9(60.0)$ & $1(6.7)$ & $1(6.7)$ & $1(6.7)$ & $3(20.0)$ & $1(6.7)$ \\
\hline $\begin{array}{l}\text { Dry vagina } \\
\text { (only for women, } n=24 \text { ) }\end{array}$ & $12(50)$ & $1(4.2)$ & $3(12.5)$ & $4(16.7)$ & $1(4.2)$ & $3(12.5)$ \\
\hline
\end{tabular}

${ }^{a}$ Based on questions of the Chemotherapy Induced Neurotoxicity Questionnaire. ${ }^{b} \mathrm{~N}=38$ because not all patients completed the questionnaire. 
Table 2.4 Comparison of FACT/GOG well-being subscales between patients with low or high neuropathy symptoms on the FACT/GOG-Ntx.

\begin{tabular}{|c|c|c|c|}
\hline \multirow[b]{2}{*}{ Well-being subscale } & \multicolumn{3}{|c|}{ Mean score (SD) } \\
\hline & Low $^{a}$ & High $^{b}$ & $p$ value \\
\hline Physical & $23.0(4.6)$ & $21.6(5.0)$ & .972 \\
\hline Social/familial & $19.5(3.8)$ & $20.6(2.7)$ & .398 \\
\hline Emotional & $6.2(2.8)$ & $8.9(3.3)$ & .002 \\
\hline Functional & $17.8(6.5)$ & $15.5(5.5)$ & .636 \\
\hline
\end{tabular}

FACT/GOG-Ntx, Functional Assessment of Cancer Therapy/Gynecologic Oncology Group-Neurotoxicity.

${ }^{\mathrm{a}}$ Low scores $=75 \%$ of patients with lowest scores on FACT/GOG-Ntx subscale. ${ }^{b}$ High scores $=$ upper $25 \%$ of patients with the highest scores on FACT/GOG-Ntx subscale indicating worse neuropathy symptoms.

\section{Discussion}

This study indicates the occurrence of CIPN and a decrease in QoL because of CIPN in $48.6 \%$ of patients after a median follow up of 6.5 months after cessation of chemotherapy. Most of the patients reported symptoms of CIPN in the upper and lower extremities (78.8\% and $89.7 \%$, respectively). Overall, most reported complaints included numbness and tingling in hands and feet, suffering from cold feet, and difficulty distinguishing objects in the hands. Patients were limited in their daily activities because of these symptoms, and a number of them stated that they became more dependent on others because of the neurotoxicity. Patients who reported many neuropathy symptoms reported significantly worse emotional well-being than did the patients with fewer neuropathy symptoms.

With the increase in the use of chemotherapy in treating cancer, CIPN is becoming a major survivorship issue that can have a negative impact on QoL. However, the few studies that have directly investigated the effects of this side-effect on QoL were highly diverse and used different assessment tools. ${ }^{15}$ In addition, a comparison of studies that used the same assessment tools for CIPN and QoL still presents problems because they include different patient populations, have different follow-up times, and the results were presented in different ways. For instance, a study of 100 patients that used the FACT/GOG-Ntx questionnaire reported any degree of neurotoxicity in $80 \%$ of the breast cancer patients at a median follow-up of 12 months (range, 6-24 months) and a higher neurotoxicity score was likely to be associated with lower physical well-being on the FACT-G. ${ }^{22}$ Another study also showed that neuropathy symptoms were significantly associated with worse QoL as demonstrated by significantly worse FACT-G subscale scores in 230 patients with non-small-cell lung cancer 6 and 12 weeks after cessation of chemotherapy. ${ }^{16}$ Two studies, which included cancer patients with different types of malignancies and chemotherapy treatments, also showed worse QoL in patients with symptoms of CIPN after treatment, ${ }^{1,21}$ whereas another study with 99 ovarian cancer patients did not find a 
relation between CIPN and global QoL. ${ }^{12}$ We demonstrated the occurrence and severity of CIPN and the impact of CIPN on global QoL and emotional well-being median 6 months after cessation of chemotherapy. Our results confirm those of previous studies that also showed a mainly negative impact of CIPN symptoms on QoL. However, comparison with these studies is complicated for aforementioned reasons. Patients with remaining complaints after 6 months are at risk of having irreversible CIPN and recognition of symptoms and impact on QoL in these patients is therefore necessary.

This study has some limitations that should be mentioned. We used the relatively new developed and informative CINQ, which reports about many neuropathy symptoms, including the sensory, motor, and autonomic nervous system symptoms, and evaluates the influence of CIPN on QoL. ${ }^{1}$ However, the CINQ requires further validation and therefore we used the validated FACT/GOG-Ntx for concomitant evaluation of CIPN and QoL, which showed the same common symptoms and interference of CIPN on QoL after median 6.5 months after cessation of therapy. We have combined the answers somewhat, quite a bit, and very much of the FACT/GOG$\mathrm{Ntx}$ to demonstrate the occurrence of CIPN, as a patient who states to have somewhat complaints of CIPN has relevant symptoms. In addition, because of central tendency bias, ${ }^{28}$ only a few patients will respond to the questions with very much. In our study with its relatively small sample size, selection bias cannot be ruled out because it is not known whether nonrespondents, for example, declined to fill out our questionnaire because they were prevented from doing so because of neuropathy symptoms in their hands or because of the lack of neuropathy and bias from unanswered questions (5\%-10\%) might have influenced the outcomes. Questionnaires were obtained after median 6.5 months only, so information about symptoms during the chemotherapy lacks. Objective measures as neurological examination or nerve conduction studies were not carried out, as there is a lack of consensus on the value of these objective measures in daily practice ${ }^{28-30}$ and they do not always correspond to subjective complaints and influence on QoL.

In summary, we believe that our results may contribute to the limited data available on the impact of CIPN on QoL after 6 months. The assessment of this impact not only with the FACT/GOG-Ntx, but also with the CINQ may be of additional value, although the CINQ needs further validation. In other studies however, investigators used different questionnaires that were completed during longer follow-up periods. Recognition of CIPN in patients after 6 months is needed because of the importance of these patients being informed and guided in regard to their condition. CIPN is becoming a major survivorship issue, especially because its prevention and treatment remains difficult. Therefore, more research concerning the prevention and treatment of CIPN is required. In addition, it is important that consensus will be reached in the assessment of CIPN and QoL in cancer patients and large studies are needed to assess the impact of CIPN on QoL, both during and after treatment. 


\section{References}

1. Driessen CM, de Kleine-Bolt KM, Vingerhoets AJ, Mols F, Vreugdenhil G. Assessing the impact of chemotherapy-induced peripheral neurotoxicity on the quality of life of cancer patients: the introduction of a new measure. Support Care Cancer. 2012;20:877-881.

2. Gutiérrez-Gutiérrez G, Sereno M, Miralles A, Casado-Sáenz E, Gutiérrez-Rivas E. Chemotherapyinduced peripheral neuropathy: clinical features, diagnosis, prevention and treatment strategies. Clin Transl Oncol. 2010;12:81-91.

3. Kannarkat G, Lasher E, Schiff D. Neurologic complications of chemotherapy agents. Curr Opin Neurol. 2007;20:719-725.

4. Ocean A, Vahdat L. Chemotherapy-induced peripheral neuropathy: pathogenesis and emerging therapies. Support Care Cancer. 2004;12:619-625.

5. Quasthoff S, Hartung H. Chemotherapy-induced peripheral neuropathy. J Neurol. 2002;249:9-17.

6. Farquhar-Smith P. Chemotherapy-induced neuropathic pain. Curr Opin Support Palliat Care. 2011;5: 1-7.

7. Beijers AJ, Jongen JL, Vreugdenhil G. Chemotherapy-induced neurotoxicity: the value of neuroprotective strategies. Neth J Med. 2012;70:18-25.

8. Wolf S, Barton D, Kottschade L, Grothey A, Loprinzi C. Chemotherapy-induced peripheral neuropathy: prevention and treatment strategies. Eur J Cancer. 2008;44:1507-1515.

9. Extra JM, Marty M, Brienza S, Misset JL. Pharmacokinetics and safety profile of oxaliplatin. Semin Oncol. 1998;25(2 Suppl 5):13-22.

10. Pachman DR, Barton DL, Watson JC, Loprinzi CL. Chemotherapy-induced peripheral neuropathy: prevention and treatment. Clin Pharmacol Ther. 2011;90:377-387.

11. Finnerup NB, Sindrup SH, Jensen TS. The evidence for pharmacological treatment of neuropathic pain. Pain. 2010;150:573-581.

12. Calhoun EA, Welshman EE, Chang $\mathrm{CH}$, et al. Psychometric evaluation of the Functional Assessment of Cancer Therapy/Gynecologic Oncology Group-Neurotoxicity (Fact/GOG-Ntx) questionnaire for patients receiving systemic chemotherapy. Int J Gynecol Cancer. 2003;13:741-748.

13. Cella DF, Tulsky DS, Gray G, et al. The Functional Assessment of Cancer Therapy scale: development and validation of the general measure. J Clin Oncol. 1993;11:570-579.

14. Postma TJ, Aaronson NK, Heimans JJ, et al. The development of an EORTC quality of life questionnaire to assess chemotherapy-induced peripheral neuropathy: the QLQ-CIPN20. Eur J Cancer. May 2005;41:1135-1139.

15. Mols F, Beijers AJM, Vreugdenhil G, Van de Poll-Franse LV. Chemotherapy-induced peripheral neuropathy and its association with quality of life: A systematic review. Support Care Cancer. 2014;22:2261-2269.

16. Cella D, Peterman A, Hudgens S, Webster K, Socinski MA. Measuring the side effects of taxane therapy in oncology: the functional assesment of cancer therapy-taxane (FACT-taxane). Cancer. 2003;98:822-831.

17. Kim BJ, Park HR, Roh HJ, et al. Chemotherapy-related polyneuropathy may deteriorate quality of life in patients with B-cell lymphoma. Qual Life Res. 2010;19:1097-1103.

18. Ostchega $\mathrm{Y}$, Donohue M, Fox N. High-dose cisplatin-related peripheral neuropathy. Cancer Nurs. 1988;11:23-32.

19. Sorbe B, Graflund M, Nygren L, et al. A phase II study of docetaxel weekly in combination with carboplatin every three weeks as first line chemotherapy in stage IIB-IV epithelial ovarian cancer: neurological toxicity and quality-of-life evaluation. Int J Oncol. 2012;40:773-781.

20. Mols F, Beijers T, Lemmens V, van den Hurk CJ, Vreugdenhil G, van de Poll-Franse LV. Chemotherapyinduced neuropathy and its association with quality of life among 2- to 11-year colorectal cancer survivors: results from the population-based PROFILES registry. J Clin Oncol. 2013;31:2699-2707.

21. Griffith KA, Couture DJ, Zhu S, et al. Evaluation of chemotherapy-induced peripheral neuropathy using current perception threshold and clinical evaluations. Support Care Cancer. 2014;22:1161-1169. 
22. Hershman DL, Weimer LH, Wang A, et al. Association between patient reported outcomes and quantitative sensory tests for measuring long-term neurotoxicity in breast cancer survivors treated with adjuvant paclitaxel chemotherapy. Breast Cancer Res Treat. 2011;125:767-774.

23. Morita S, Kobayashi K, Eguchi K, et al. Influence of clinical parameters on quality of life during chemotherapy in patients with advanced non-small cell lung cancer: application of a general linear model. Jpn J Clin Oncol. 2003;33:470-476.

24. Ramchandren S, Leonard M, Mody RJ, et al. Peripheral neuropathy in survivors of childhood acute lymphoblastic leukemia. J Peripher Nerv Syst. 2009;14:184-189.

25. Park SB, Lin CS, Krishnan AV, Goldstein D, Friedlander ML, Kiernan MC. Long-term neuropathy after oxaliplatin treatment: challenging the dictum of reversibility. Oncologist. 2011;16:708-716.

26. Tofthagen C. Patient perceptions associated with chemotherapy-induced peripheral neuropathy. Clin J Oncol Nurs. 2010;14:E22-28.

27. Huang HQ, Brady MF, Cella D, Fleming G. Validation and reduction of FACT/GOG-Ntx subscale for platinum/paclitaxel-induced neurologic symptoms: a gynecologic oncology group study. Int J Gynecol Cancer. 2007;17:387-393.

28. Cavaletti G, Frigeni B, Lanzani F, et al. Chemotherapy-Induced Peripheral Neurotoxicity assessment: a critical revision of the currently available tools. Eur J Cancer. 2010;46:479-494.

29. Postma TJ, Heimans JJ, Muller MJ, Ossenkoppele GJ, Vermorken JB, Aaronson NK. Pitfalls in grading severity of chemotherapy-induced peripheral neuropathy. Annal Oncol. 1998;9:739-744.

30. Cavaletti G, Cornblath DR, Merkies IS, et al. The chemotherapy-induced peripheral neuropathy outcome measures standardization study: from consensus to the first validity and reliability findings. Annal Oncol. 2013;24:454-462. 


\section{Chapter 3}

Chemotherapy-induced neuropathy and its association with quality of life among 2- to 11-year colorectal cancer survivors: Results from the population-based PROFILES registry

Mols F, Beijers T, Lemmens V, van den Hurk CJ, Vreugdenhil G, van de Poll-Franse LV 


\section{Abstract}

\section{Purpose}

To gain insight into the prevalence and severity of chemotherapy-induced neuropathy and its influence on health-related quality of life (HRQOL) in a population-based sample of colorectal cancer (CRC) survivors 2 to 11 years after diagnosis.

\section{Methods}

All alive individuals diagnosed with CRC between 2000 and 2009 as registered by the Dutch population-based Eindhoven Cancer Registry were eligible for participation. Eighty-three percent $(n=1,643)$ of patients filled out the European Organisation for Research and Treatment of Cancer (EORTC) Quality of Life Questionnaire (QLQ) C30 and the EORTC QLQ ChemotherapyInduced Peripheral Neuropathy 20.

\section{Results}

The five neuropathy-subscale related symptoms that bothered patients with CRC the most during the past week were erectile problems ( $42 \%$ of men), trouble hearing $(11 \%)$, trouble opening jars or bottles (11\%), tingling toes/feet (10\%), and trouble walking stairs or standing up (9\%). Additionally, patients who received oxaliplatin more often reported tingling ( $29 \%$ vs. $8 \%$; $p=0.001)$, numbness ( $17 \%$ vs. $5 \% ; p=0.005)$, and aching or burning pain $(13 \%$ vs. $6 \% ; p=0.03)$ in toes/feet compared to those not treated with chemotherapy. They also more often reported tingling toes/feet ( $29 \%$ vs. $14 \% ; p=0.0127$ ) compared with those treated with chemotherapy without oxaliplatin. Those with many neuropathy symptoms (e.g. upper 10\%) reported statistically significant and clinically relevant worse HRQOL scores on all EORTC QLQ-C3O subscales (all $p<0.01$ ).

\section{Conclusion}

Two to 11 years after diagnosis of CRC, neuropathy-related symptoms are still reported, especially sensory symptoms in the lower extremities among those treated with oxaliplatin. Because neuropathy symptoms have a negative influence on HRQOL, these should be screened for and alleviated. Future studies should focus on prevention and relief of chemotherapyinduced neuropathy. 


\section{Introduction}

Colorectal cancer (CRC) is the third most common cause of cancer among men and women in the Western world. ${ }^{1}$ In 2007, 12,000 patients were diagnosed with CRC in the Netherlands, and this number is expected to increase to 17,000 in $2020 .^{2}$ Simultaneously, the 10 -year prevalence will increase with $56 \%$, from 59,000 to $92,000,{ }^{2}$ mainly because of the aging of the population and improved survival rates. The current 5-year survival rate for CRC in the Netherlands is $59 \%{ }^{3}$

As a result of the increasing prevalence, more patients are living with the long-term adverse effects of CRC and its treatment. The development of neuropathy, a common adverse effect of the platinum agent oxaliplatin, is especially a major concern that may negatively influence a patients' health-related quality of life (HRQOL). ${ }^{4}$ Oxaliplatin, in combination with fluorouracil or its oral analogs, is now the regimen of choice for adjuvant treatment of patients with node-positive CRC., ${ }^{5,6}$ Treatment and

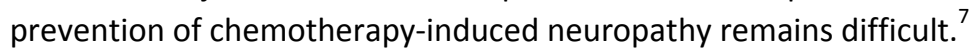

Acute neuropathy, which is often cold-triggered with distal paresthesias, dysesthesias and mild muscle contractions of hands, feet and perioral region, occurs most frequently. ${ }^{7}$ This reverses in up to $90 \%$ of cases after oxaliplatin is discontinued. ${ }^{5}$ However, a significant proportion of patients experience chronic neuropathy, which is mainly sensory. ${ }^{5,8,9}$ An Australian study reported that neuropathy persisted in $79 \%$ of patients after a median follow-up of 29 months. ${ }^{10}$ Furthermore, a European trial showed that $26 \%$ of patients with grade 3 neurotoxicity had persistent symptoms after a median of 28 months of follow-up. ${ }^{8}$ Additionally, more than $10 \%$ of patients in the National Surgical Adjuvant Breast and Bowel Project C-07 trial had persistent neuropathy 2 years after completing therapy, ${ }^{9}$ whereas the MOSAIC (Multicenter International Study of Oxaliplatin/Fluorouracil/Leucovorin in the Adjuvant Treatment of Colon Cancer) trial reported a grade 1 to 3 neuropathy prevalence of $30 \% 1$ year after treatment and $24 \%$ after 18 months. ${ }^{5}$ The previously mentioned studies show that the prevalence of chronic neuropathy varies significantly between studies which is most likely caused by the limitations of current grading scales and the absence of consensus on the gold standard for symptom assessment. ${ }^{11-13}$ Because of the increasing prevalence of CRC and the increased use of oxaliplatin, it is likely that neuropathy will become a major cancer survivorship issue.

Chemotherapy-induced peripheral neuropathy can be severe, may result in serious limitations in daily functioning, and might therefore have a negative impact on HRQOL. Nonetheless, the literature on the association between neuropathy and $\mathrm{HRQOL}$ is scarce. Besides, the existing studies are small, and the use of validated HRQOL questionnaires is low. More importantly, most studies have focussed on acute instead of chronic neuropathy, whereas a large proportion of patients with CRC will become long-term survivors and neuropathy therefore might be a chronic problem. 
Therefore, our aim was to gain insight into the prevalence, severity, and symptoms of chemotherapy-induced neuropathy among a population-based sample of CRC survivors, up to 11 years after diagnosis. Chemotherapy-induced neuropathy was assessed with a self-report instrument to determine its burden on daily life. In addition, the impact of neuropathy on HRQOL was studied.

\section{Methods}

\section{Setting and participants}

Data collection was performed within PROFILES (Patient Reported Outcomes Following Initial Treatment and Long Term Evaluation of Survivorship), which is a registry for the physical and psychosocial impact of cancer and its treatment from a dynamic, growing population-based cohort of cancer survivors. ${ }^{14}$ PROFILES contains a large web-based component and is linked directly to the Eindhoven Cancer Registry (ECR), which compiles data of all individuals newly diagnosed with cancer in the southern part of the Netherlands. ${ }^{15}$ Data from the PROFILES registry will be available for noncommercial scientific research, subject to study question, privacy and confidentiality restrictions, and registration (www.profilesregistry.nl).

Our survey was set up in December 2010. All individuals diagnosed with CRC between 2000 and 2009 as registered in the ECR were eligible for participation (except those already included in a 2009 PROFILES survey ${ }^{16,17}$ ). Patients who had cognitive impairment, had died prior to start of the study (according to the ECR; the Central Bureau for Genealogy which collects information on all deceased Dutch citizens via the civil municipal registries; and hospital records), or had unverifiable addresses were excluded. One year later, the second data collection wave took place, which included a questionnaire on neuropathy. The data presented in this article are based upon this second wave. This study was approved by a certified medical ethics committee. All patients signed informed consent.

\section{Data collection}

Survivors were informed of the study via a letter from their (ex-)attending specialist. Nonrespondents were sent a reminder within 2 months.

\section{Sociodemographic and clinical characteristics}

Survivors' sociodemographic and clinical information was available from the ECR, which routinely collects data such as date of diagnosis, tumor grade, ${ }^{18}$ clinical stage, ${ }^{18}$ and treatment. Comorbidity at time of the study was assessed with the adapted Self- 
administered Comorbidity Questionnaire. ${ }^{19}$ Socioeconomic status was determined by an indicator developed by Statistics Netherlands. ${ }^{20}$ Questions on marital status, educational level, and current occupation were added to the questionnaire.

\section{Health-related quality of life}

The European Organisation for Research and Treatment of Cancer (EORTC) Quality of Life Questionnaire (QLQ) C30 (Version 3.0) was used to assess HRQOL. ${ }^{21}$ It contains five functional scales on physical, role, cognitive, emotional and social functioning; a global health status/QOL scale; three symptoms scales on fatigue, nausea and vomiting, and pain; and six single items assessing dyspnea, insomnia, loss of appetite, constipation, diarrhea, and financial impact. Each item is scored from 1 (not at all) to 4 (very much), except for the global QOL scale, which ranges from 1 (very poor) to 7 (excellent). Scores were linearly transformed to a 0 to 100 scale $^{22}$; a higher score on the functional scales and global QOL means better functioning and QOL, whereas a higher score on the symptom scales mean more complaints.

\section{Chemotherapy-induced neuropathy}

Chemotherapy-induced neuropathy was assessed with the EORTC QLQ Chemotherapy-Induced Peripheral Neuropathy 20 (CIPN20), ${ }^{23}$ which contains three subscales assessing sensory, motor, and autonomic symptoms. Each item is measured on a Likert scale ranging from 1 (not at all) to 4 (very much). Scores were linearly transformed to a 0 to 100 scale, with higher scores representing more complaints.

\section{Statistical analyses}

ECR data on demographic and clinical characteristics enabled us to compare the group of respondents, nonrespondents, and patients with unverifiable addresses, using t-tests for continuous and chi-square analyses for categorical variables. Differences in sociodemographic and clinical characteristics between patients who did or did not receive chemotherapy treatment were analyzed in a similar way.

For the total group, responses to all questions of the EORTC QLQ-CIPN20 were shown in percentages. Because oxaliplatin has been used since 2007, logistic regression analyses were performed on this selected group of CRC patients to detect differences regarding experienced neuropathy problems in the past week (EORTC QLQ-CIPN20; answer categories "quite a bit" or "very much" combined) between the different treatments. Analyses were adjusted for stage, diabetes, osteoarthritis, and rheumatoid arthritis which are comorbid conditions that are known to be associated with neuropathy-like symptoms.

Mean scores on the EORTC QLQ-CIPN20 subscales, stratified by treatment, were compared with analyses of covariance for patients with colon and rectal cancer 
diagnosed since 2007. Confounding background variables included for adjustment in these analyses were determined a priori $^{24}$ and chosen to be stage, diabetes, osteoarthritis and rheumatoid arthritis. Clinically meaningful differences were determined with Norman's rule of thumb, whereby a difference of $\approx 0.5$ SD indicates a threshold of discriminant change. ${ }^{25}$ In addition, multivariate linear regression models were used to investigate the association between sociodemographic and clinical characteristics with the EORTC QLQ-CIPN20 subscales and the most important single questions of the sensory scale.

Finally, among the total group of CRC survivors, a comparison of EORTC QLQ-C30 subscale scores between those who reported low or high (eg, top 10\% of patients) sensory symptoms was made with analysis of covariance. Confounding background variables included for adjustment were determined a priori ${ }^{24}$ and chosen to be age at time of questionnaire, years since diagnosis, marital status, stage, number of comorbid conditions, diabetes, osteoarthritis, and rheumatoid arthritis. Clinically important differences were determined on EORTC QLQ-C30 guidelines. ${ }^{26}$

All test were two-sided, considered significant if $p<0.05$, and were performed using SAS (version 9.2 for Windows, SAS institute Inc., Cary NC).

\section{Results}

\section{Sociodemographic and clinical characteristics}

Data collected during the second data wave of a study among CRC survivors was used, which had a response rate of $83 \%(n=1,643)$. There were no significant differences between respondents, nonrespondents and those with nonverified addresses with respect to sociodemographic and clinical characteristics (Table 3.1). Of the total group of respondents, 500 patients (31\%) were treated with chemotherapy and they were significantly younger, diagnosed more recently, and more often diagnosed with colon instead of rectal cancer compared to those not treated with chemotherapy (Table 3.2). Furthermore, they had a higher disease stage and grade at diagnosis, reported a higher body mass index, and were more often employed at the time of survey. 
Table 3.1 Demographic and clinical characteristics of selected sample by response status.

\begin{tabular}{|c|c|c|c|c|}
\hline Characteristics & $\begin{array}{c}\text { Respondents } \\
(n=1,643,83 \%) \\
n(\%)\end{array}$ & $\begin{array}{c}\text { Non-respondents } \\
(n=322,16 \%) \\
n(\%)\end{array}$ & $\begin{array}{l}\text { Non-verified } \\
\text { addresses } \\
(n=5,0.3 \%) \\
n(\%)\end{array}$ & p-value \\
\hline Mean age at time of survey $( \pm S D)$ & $69.4(9.4)$ & $69.4(9.9)$ & $75(9.7)$ & 0.3530 \\
\hline Mean years since last diagnosis $( \pm S D$ ) & $5.9(2.8)$ & $5.7(2.6)$ & $7.4(3.7)$ & 0.2789 \\
\hline \multicolumn{5}{|l|}{ Sex } \\
\hline Male & $935(57)$ & $183(57)$ & $3(60)$ & 0.9899 \\
\hline Female & $708(43)$ & $139(43)$ & $2(40)$ & \\
\hline Location of tumor & & & & 0.62 \\
\hline Colon & $976(59)$ & $196(61)$ & $4(80)$ & \\
\hline Rectum & $655(40)$ & $126(39)$ & $1(20)$ & \\
\hline \multicolumn{4}{|l|}{ TNM stage } & 0.2405 \\
\hline I & $481(29)$ & $97(30)$ & $0(0)$ & \\
\hline II & $571(35)$ & $124(39)$ & $3(60)$ & \\
\hline III & 484 (29) & $74(23)$ & $2(40)$ & \\
\hline \multirow{2}{*}{ Unknown } & $53(3)$ & $16(5)$ & $0(0)$ & \\
\hline & $54(4)$ & $11(3)$ & $0(0)$ & \\
\hline \multicolumn{4}{|l|}{ Tumour differentiation grade } & 0.1314 \\
\hline 1 & $153(9)$ & $18(6)$ & $0(0)$ & \\
\hline 2 & $987(61)$ & $192(60)$ & $5(100)$ & \\
\hline 3 & $177(11)$ & $38(12)$ & $0(0)$ & \\
\hline Unknown & 314 (19) & $74(23)$ & $0(0)$ & \\
\hline \multicolumn{4}{|l|}{ Primary treatment } & 0.741 \\
\hline Surgery only & $744(46)$ & $166(52)$ & $1(20)$ & \\
\hline Surgery and radiotherapy & $386(24)$ & $75(23)$ & $1(20)$ & \\
\hline Surgery and chemotherapy & $354(22)$ & $57(18)$ & $3(60)$ & \\
\hline \multirow{3}{*}{$\begin{array}{l}\text { Surgery, chemotherapy, and radiotherapy } \\
\text { Chemotherapy only } \\
\text { Radiotherapy only }\end{array}$} & 139 (9) & $19(6)$ & $0(0)$ & \\
\hline & $5(0)$ & $2(1)$ & $0(0)$ & \\
\hline & $0(0)$ & $1(0)$ & $0(0)$ & \\
\hline
\end{tabular}

SD, standard deviation.

Table 3.2 Sociodemographic and clinical characteristics of colorectal cancer survivors, stratified by chemotherapy treatment.

\begin{tabular}{lccc}
\hline Characteristics & $\begin{array}{c}\text { Chemotherapy } \\
(\mathbf{n = 5 0 0 , 3 1 \% )} \\
\mathbf{n}(\%)\end{array}$ & $\begin{array}{c}\text { No chemotherapy } \\
(\mathbf{n = 1 , 1 3 1 , 6 9 \% )} \\
\mathbf{n}(\%)\end{array}$ & p-value \\
\hline Age at time of survey (mean, SD) & $66.7(9.8)$ & $70.6(9)$ & $<0.001$ \\
Age at time of survey & $208(42)$ & $340(30)$ & $<0.001$ \\
$<60$ years & $202(40)$ & $427(38)$ & \\
60-69 years & $90(18)$ & $364(32)$ & \\
$>70$ years & $5.5(2.8)$ & $6.0(2.8)$ & 0.0018 \\
Years since diagnosis (mean, SD) & & & \\
Years since diagnosis & $280(56)$ & $525(46)$ & 0.0004 \\
1-5 years & $220(44)$ & $606(54)$ & 0.5951 \\
5-10 years & & & \\
Sex & $290(58)$ & $640(57)$ & \\
Male & $210(42)$ & $491(43)$ &
\end{tabular}


Table 3.2 (continued)

\begin{tabular}{|c|c|c|c|}
\hline Characteristics & $\begin{array}{c}\text { Chemotherapy } \\
(n=500,31 \%) \\
n(\%)\end{array}$ & $\begin{array}{c}\text { No chemotherapy } \\
(n=1,131,69 \%) \\
n(\%)\end{array}$ & p-value \\
\hline Location of tumor & & & $<0.001$ \\
\hline Colon & $336(67)$ & $640(57)$ & \\
\hline Rectal & $164(33)$ & $491(43)$ & \\
\hline TNM stage & & & $<0.001$ \\
\hline 1 & $27(5)$ & $455(40)$ & \\
\hline II & $72(14)$ & $499(44)$ & \\
\hline III & $350(70)$ & $134(12)$ & \\
\hline IV & $38(8)$ & $15(1)$ & \\
\hline Unknown & $13(3)$ & $28(3)$ & \\
\hline Tumor differentiation grade & & & 0.005 \\
\hline 1 & $46(9)$ & $107(10)$ & \\
\hline 2 & $303(61)$ & $684(61)$ & \\
\hline 3 & $72(14)$ & $105(9)$ & \\
\hline Unknown & $79(16)$ & $235(21)$ & \\
\hline No. of comorbid conditions & & & 0.1442 \\
\hline None & $164(33)$ & $330(29)$ & \\
\hline 1 & $154(31)$ & $333(29)$ & \\
\hline $2+$ & $182(36)$ & $468(41)$ & \\
\hline \multicolumn{4}{|l|}{ Most frequent conditions } \\
\hline High blood pressure & $162(32)$ & $396(35)$ & 0.3050 \\
\hline Osteoarthritis & $104(21)$ & $287(25)$ & 0.0460 \\
\hline Back pain & $104(21)$ & $266(24)$ & 0.2267 \\
\hline Heart disease & $54(11)$ & $192(17)$ & 0.0013 \\
\hline Diabetes mellitus & $68(14)$ & $150(13)$ & 0.8535 \\
\hline $\mathrm{BMI}, \mathrm{kg} / \mathrm{m}^{2}$ & & & 0.0133 \\
\hline$<18.4$ (underweight) & $3(1)$ & $10(1)$ & \\
\hline 18.5 to 24.9 (normal) & $146(29)$ & $418(38)$ & \\
\hline 25 to 29.9 (overweight) & $251(50)$ & $498(45)$ & \\
\hline$>30$ (obese) & $98(20)$ & $190(17)$ & \\
\hline Marital status & & & 0.0595 \\
\hline Married & $405(81)$ & $848(76)$ & \\
\hline Single/Divorced & $38(8)$ & $105(9)$ & \\
\hline Widow/Widower & $56(11)$ & $165(15)$ & \\
\hline Education level $^{+}$ & & & 0.4167 \\
\hline Low & $70(14)$ & $166(15)$ & \\
\hline Medium & $303(61)$ & $704(63)$ & \\
\hline High & $125(25)$ & $247(22)$ & \\
\hline Current occupation status & & & 0.0007 \\
\hline Employed & $113(23)$ & $172(16)$ & \\
\hline Not employed/retired & $378(77)$ & $907(84)$ & \\
\hline Socioeconomic status & & & 0.2878 \\
\hline Low & $82(17)$ & $223(21)$ & \\
\hline Medium & $200(42)$ & $435(40)$ & \\
\hline High & $196(41)$ & 425 (39) & \\
\hline
\end{tabular}

Some variables exceed $100 \%$ due to rounding off. BMI, body mass index; SD, standard deviation. + Education: Low (no or primary school); Medium (lower general secondary education or vocational training); High (pre-university education, high vocational training, university). 


\section{Chemotherapy-induced neuropathy}

Among the total group diagnosed 2 to 11 years ago, the five neuropathy-subscale related symptoms that bothered them the most during the past week were trouble getting or maintaining an erection (42\% of men), trouble hearing (11\%), trouble opening a jar or bottle because of loss of strength in hands (11\%), tingling toes or feet $(10 \%)$, and trouble walking stairs or standing up from a chair due to weakness in legs (9\%; Table 3.3).

Logistic regression analyses among patients with CRC diagnosed from 2007 onward (e.g. after the introduction of oxaliplatin) showed that those treated with oxaliplatin more often reported tingling ( $29 \%$ vs. $8 \%$; $p=0.001)$, numbness ( $17 \%$ vs. $5 \%$; $\mathrm{p}=0.0047)$, and aching or burning pain $(13 \%$ vs. $6 \%$; $=0.0293)$ in toes or feet compared with those not treated with chemotherapy (Table 3.4). In addition, those treated with oxaliplatin more often reported tingling toes or feet ( $29 \%$ vs. $14 \%$; $p=0.0127$ ) compared with those treated with chemotherapy without oxaliplatin.

Analyses among patients with colon cancer diagnosed since 2007 showed that patients treated with oxaliplatin reported significantly higher scores on the EORTC QLQ-CIPN20 sensory scale problems compared with those not treated with chemotherapy (Figure 3.1). Furthermore, patients treated with chemotherapy without oxaliplatin scored significantly higher than those not treated with chemotherapy. These differences were clinically relevant ${ }^{25,26}$. In contrast, no differences between treatments were found on the motor and autonomic scales. Similar analyses among patients with rectal cancer showed that those treated with oxaliplatin reported significantly higher scores on both the sensory and motor scale compared with those treated with chemotherapy without oxaliplatin. These differences were however not clinically relevant.

Multivariate linear regression analyses evaluating the association of sociodemographic (sex, age) and clinical (years since diagnosis, tumor type, treatment, stage, body mass index, diabetes, osteoarthritis and rheumatoid arthritis) characteristics of CRC survivors with the EORTC QLQ-CIPN20 subscales showed that oxaliplatin was positively associated with the sensory scale (standardized $\beta=0.15$; $\mathrm{p}<0.001$ ). Similarly, these analyses were done on the most important single questions of the sensory scale, which showed that oxaliplatin was positively associated with tingling $(\beta=0.20 ; p<0.001)$, numbness $(\beta=0.18 ; p<0.001)$ and aching or burning pain $(\beta=0.10 ; p<0.001)$ in toes or feet. 


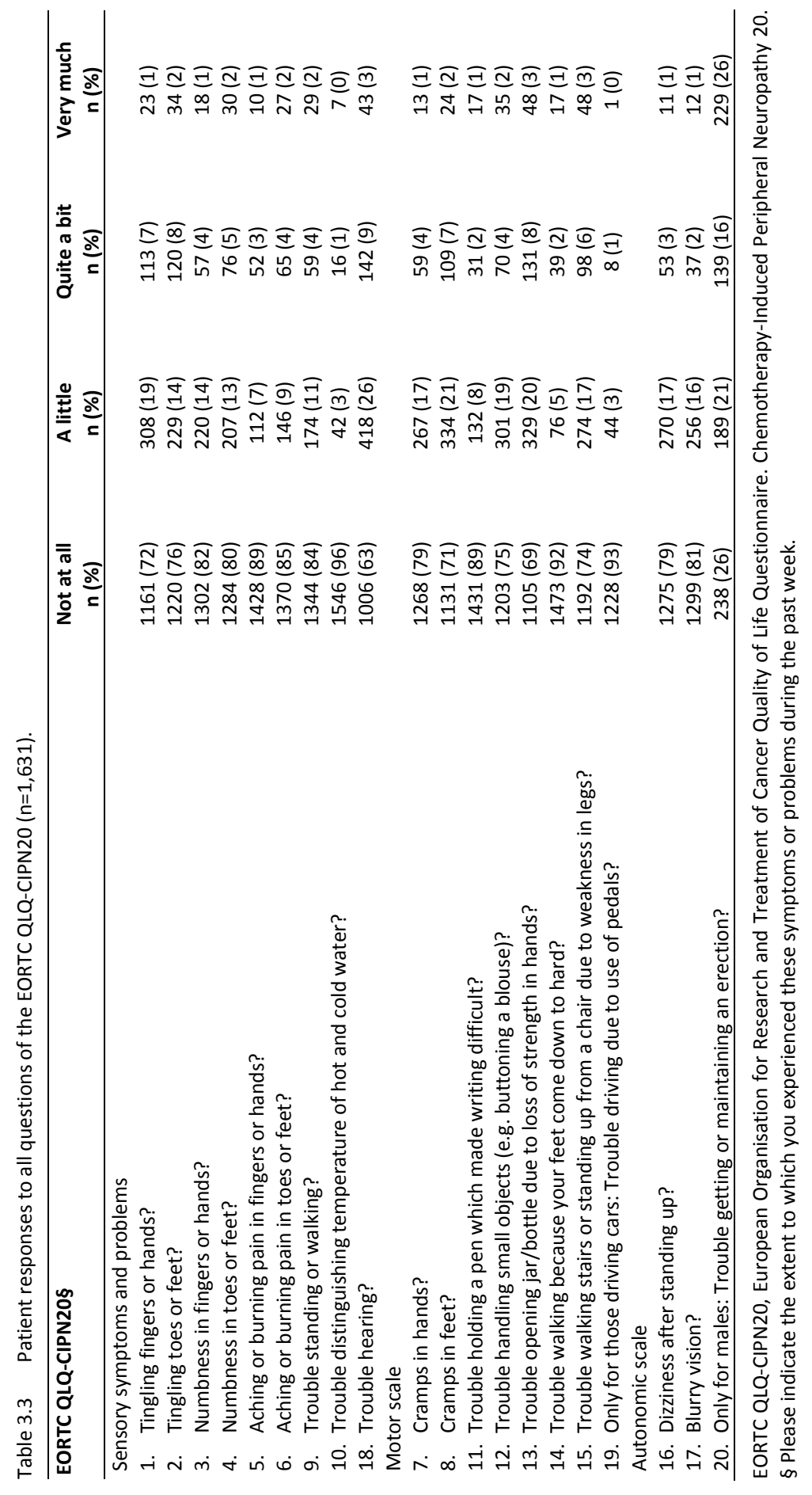




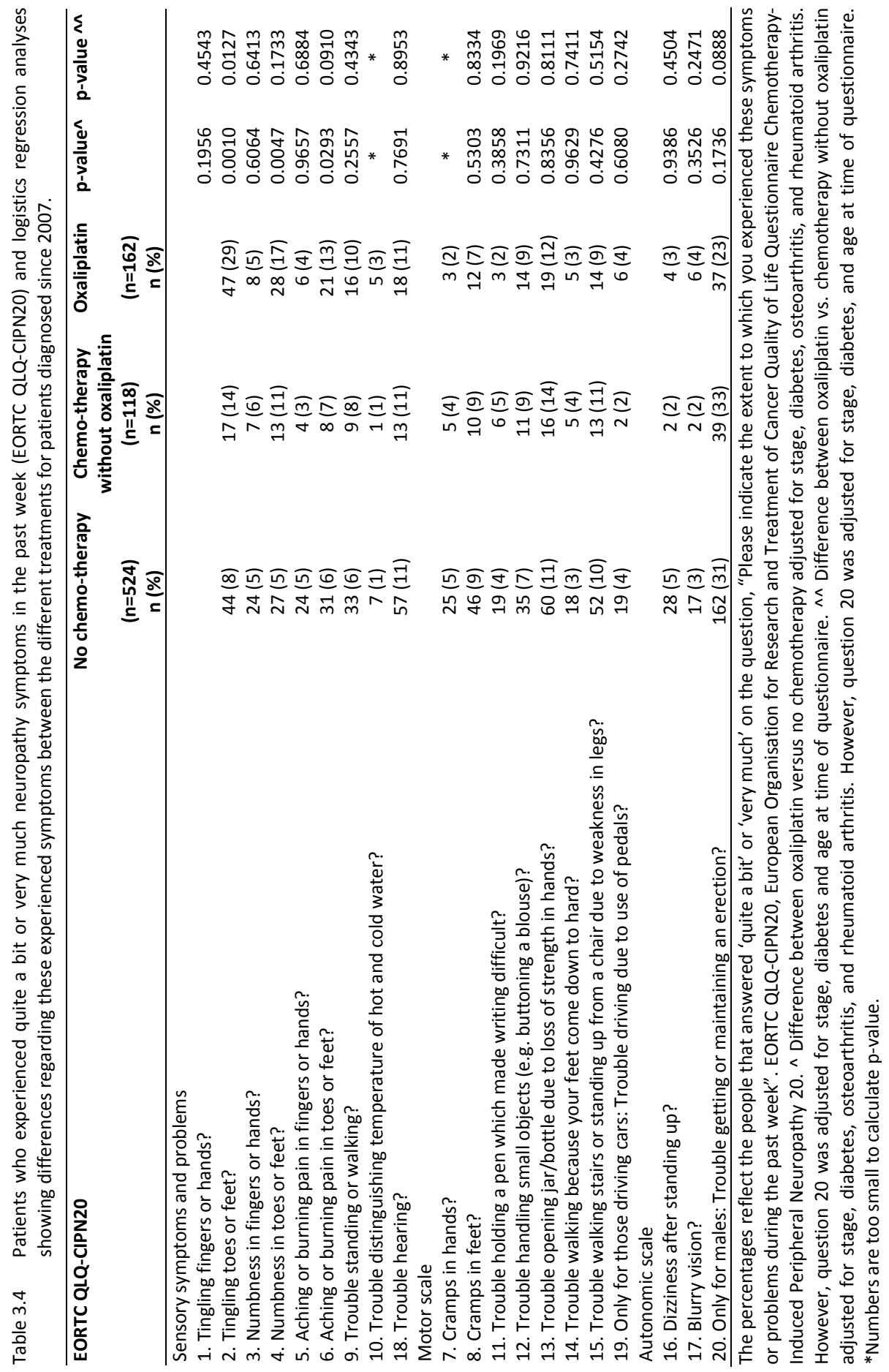




\section{Colon cancer survivors}

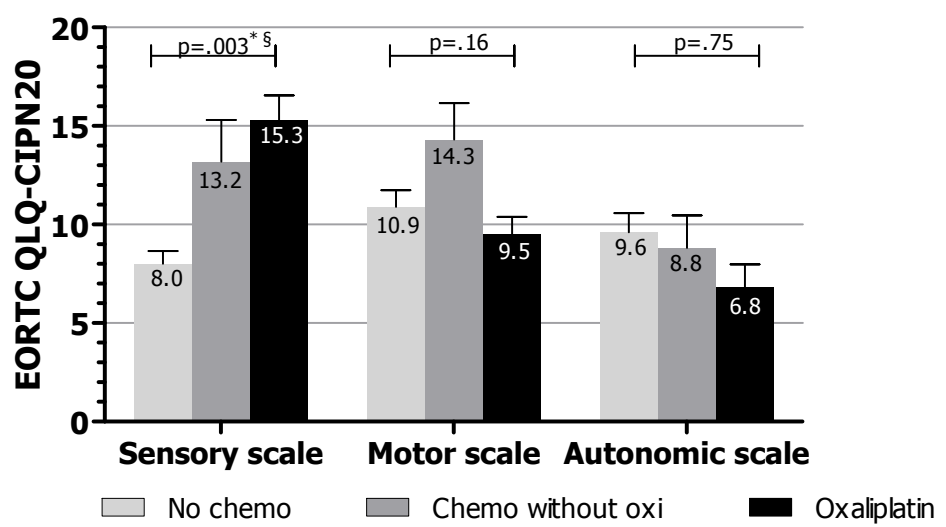

\section{Rectal cancer survivors}

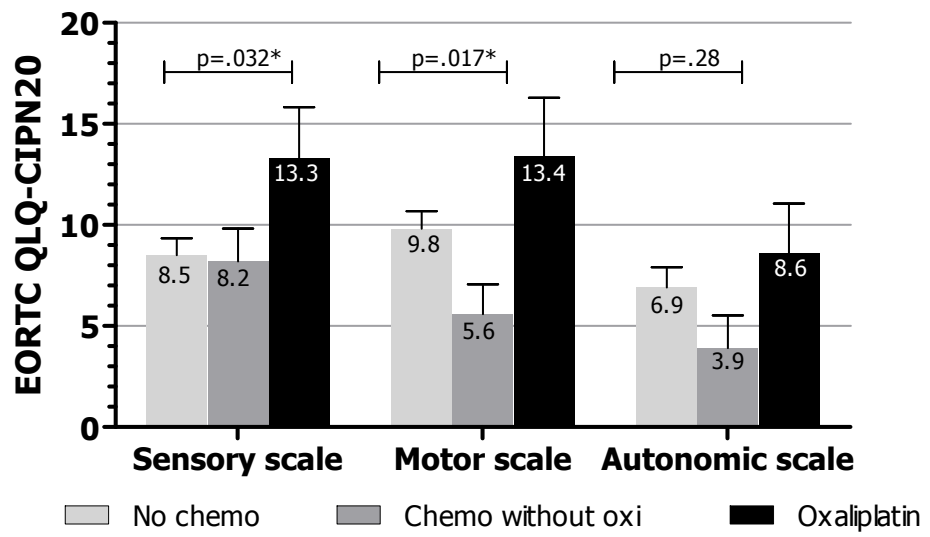

Figure 3.1 Mean scores on the EORTC QLQ-CIPN20 of patients with colon $(n=493)$ and rectal $(n=291)$ cancer, stratified by chemotherapy (since 2007). Confounding background variables included for adjustment in these analyses were determined a priori ${ }^{24}$ and chosen to be diabetes, osteoarthritis, and rheumatoid arthritis. NOTE. No significant differences between chemotherapy without oxaliplatin and oxaliplatin were found. * Significant difference between no chemotherapy and chemotherapy without oxaliplatin, and between no chemotherapy and oxaliplatin. § Clinically relevant difference based on Norman's rule of thumb of half a standard deviation. Standard deviations for colon cancer survivors; sensory scale: $11.3,17.6,13.9$, respectively; motor scale 14.6, 15.5, 9.9, respectively; autonomic scale: $16.9,13.9,13.1$, respectively. Standard deviations for rectal cancer survivors; sensory scale: $12.2,10.8,15.4$, respectively; motor scale: $12.6,9.7,17.6$, respectively; autonomic scale: 14.7 , $10.8,15.0$, respectively. 


\section{Health-related quality of life}

No significant differences were found regarding the EORTC QLQ-C30 subscale scores between CRC survivors diagnosed since 2007 who were not treated with chemotherapy, and those treated with chemotherapy with or without oxaliplatin (data not shown). However, for the total group of CRC patients, EORTC QLQ-C30 subscale scores between those who reported many sensory neuropathy symptoms (eg, upper $10 \%$ of scores) and those who reported less symptoms (eg, the other $90 \%$ ) were compared (Table 3.5). Results showed that those with many neuropathy symptoms reported significantly lower and thus worse scores on all functioning scales and the global health status scale. In addition, these patients reported higher and thus worse scores on all symptoms scales. These results were all clinically relevant, ranging from a small to large clinically important difference.

Table 3.5 Comparison of EORTC QLQ-C30 scores among CRC survivors with low or high sensory symptoms on the EORTC QLQ-CIPN20.

\begin{tabular}{|c|c|c|c|c|}
\hline EORTC QLQ-C30 ${ }^{\&}$ & $\begin{array}{c}\text { Low score on } \\
\text { sensory scale^^ } \\
(n=1,401) \\
\text { Mean (SD) }\end{array}$ & $\begin{array}{c}\text { High score on } \\
\text { sensory scale } \\
(n=153) \\
\text { Mean (SD) } \\
\end{array}$ & p-value ${ }^{\#}$ & CID* \\
\hline Physical functioning & $84(18)$ & $66(25)$ & $<0.0001$ & Large \\
\hline Role functioning & $85(23)$ & $60(32)$ & $<0.0001$ & Medium \\
\hline Emotional functioning & $89(16)$ & $76(26)$ & $<0.0001$ & Small \\
\hline Cognitive function & $87(17)$ & $73(27)$ & $<0.0001$ & Medium \\
\hline Social functioning & $90(19)$ & $74(28)$ & $<0.0001$ & Large \\
\hline Global health status/QoL & $79(17)$ & $64(23)$ & $<0.0001$ & Medium \\
\hline Fatigue & $18(21)$ & $41(29)$ & $<0.0001$ & Large \\
\hline Nausea and vomiting & $3(10)$ & $10(18)$ & $<0.0001$ & Small \\
\hline Pain & $13(21)$ & $38(33)$ & $<0.0001$ & Large \\
\hline Dyspnoea & $12(22)$ & $28(33)$ & $<0.0001$ & Large \\
\hline Insomnia & $18(25)$ & $34(33)$ & $<0.0001$ & Medium \\
\hline Loss of appetite & $4(14)$ & $14(26)$ & $<0.0001$ & Small \\
\hline Constipation & $8(18)$ & $16(25)$ & $<0.0001$ & Small \\
\hline Diarrhea & $11(21)$ & $18(25)$ & $<0.01$ & Small \\
\hline Financial impact & $5(16)$ & $16(26)$ & $<0.0001$ & Medium \\
\hline
\end{tabular}

CID, clinically important difference; EORTC QLQ-CIPN20, European Organisation for Research and Treatment of Cancer Quality of Life Questionnaire Chemotherapy-Induced Peripheral Neuropathy 20; QOL, quality of life; SD, standard deviation. ${ }^{\wedge}{ }^{\wedge}$ Low score; $90 \%$ of patients with the lowest scores. ${ }^{\wedge}$ High score; upper $10 \%$ of patients with the highest scores. ${ }^{~ C o n f o u n d i n g ~ b a c k g r o u n d ~ v a r i a b l e s ~ i n c l u d e d ~ f o r ~ a d j u s t m e n t ~}$ were determined a priori and chosen to be age at time of questionnaire, years since diagnosis, marital status, stage, number of comorbid conditions, diabetes, osteoarthritis, and rheumatoid arthritis. ${ }^{*} \mathrm{CID}$; Clinically important difference. This is based upon recently published guidelines for the EORTC QLQ-C30. ${ }^{26}$ ${ }^{\&} \mathrm{~A}$ higher score on the functional scales and global QOL means better functioning and QOL, whereas a higher score on the symptom scales means more complaints. 


\section{Discussion}

The five neuropathy subscale-related symptoms that bothered patients with CRC the most during the past week were erectile problems (men), trouble hearing, trouble opening a jar or bottle owing to loss of strength, tingling toes or feet, and trouble walking stairs or standing up. The majority of these symptoms are not necessarily related to cancer or its treatment but could also be age-related. Comparison with other studies is rather difficult because the EORTC QLQ-CIPN20 has hardly been used. However, a study did report more general symptoms such as pain or discomfort and loss of sensation in the upper and lower extremities as assessed with a semistructured interview. ${ }^{27}$ In addition, injuries secondary to numbness, muscle weakness, and loss of balance were reported.

Oxaliplatin was positively associated with the sensory scale and with tingling, numbness, and aching or burning pain in toes or feet. A recent cross-sectional multicenter study showed that sensory symptoms, as assessed with the EORTC QLQCIPN20, were more severe than motor and autonomic symptoms, and this was supported with clinical results. ${ }^{28}$ Furthermore, a study using the sensory subscale of the EORTC QLQ-CIPN20 reported more problems (eg, numbness, tingling, and shooting or burning pain) in the lower versus upper extremities as well. ${ }^{29}$ In addition, two small studies among patients with CRC showed that numbness in the hands and feet were the most constant symptoms. ${ }^{27,30}$

Because patients with rectal cancer receive a lower cumulative dose of chemotherapy compared with patients with colon cancer, differences in neuropathy-related symptoms could exist between patients with colon and rectal cancer treated with or without chemotherapy. Our results indeed showed that patients with colon cancer treated with chemotherapy demonstrated statistically significant worse sensory scores than nonchemotherapy-treated patients with colon cancer, whereas in patients with rectal cancer, these differences were found for both sensory and motor subscales. However, the differences among patients with rectal cancer were not clinically relevant, whereas they were for patients with colon cancer.

Numbness and aching/burning pain in toes and feet were significantly worse in those treated with oxaliplatin compared with patients not treated with chemotherapy. These symptoms were not different between those treated with oxaliplatin and those treated with other chemotherapy regimens. The fact that this difference was not statistically significant could be explained by the low number of patients included in the analyses (162 oxaliplatin, 118 other chemotherapy). Also, besides oxaliplatin, other chemotherapeutic agents such as vinca alkaloids, platinum compounds, and taxanes are known to cause neuropathy. ${ }^{31}$ The degree of neurotoxicity depends on the type of drug used, the duration of administration, and the cumulative dose applied. ${ }^{32,33}$ The use of combinations of chemotherapeutic agents can even strengthen neurotoxicity. ${ }^{32}$ 
Results of our study showed that those with many neuropathy symptoms reported statistically significant and clinically relevant worse scores on HRQOL. Although an increasing number of studies focus on HRQOL among patients with CRC in general, ${ }^{16,17,34,35}$ studies on the influence of neuropathy on this matter are scarce. A case report on a colon cancer survivor showed a decreased QOL owing to chronic oxaliplatin-induced neuropathy. ${ }^{36}$ Furthermore, a study among 33 patients showed that acute neuropathic symptoms most often interfered with "enjoyment of life" from the 14 -item interference scale. ${ }^{30}$ Besides, a study on 14 patients showed that neuropathic symptoms interfered with many aspects of daily life. ${ }^{27}$ Participants voiced feelings of frustration, depression, and loss of purpose as a result of having to give up enjoyable activities. Additionally, a Dutch study showed a negative impact of neuropathy on daily activities and QOL in patients with cancer treated with chemotherapy. ${ }^{37}$ Moreover, a prospective study among 32 patients with lymphoma concluded that those with polyneuropathy showed worse QOL in domains mainly associated with physical health status. ${ }^{38}$ Finally, a descriptive study among 28 patients with cancer treated with a variety of neurotoxic agents showed that having neuropathy symptoms resulted in diverse symptom patterns and degrees of physical symptoms from mild to severe, emotional distress, alterations in functional ability, and social role impairment. ${ }^{39}$

Although we had information on the demographic and clinical characteristics of nonrespondents and patients with nonverifiable addresses, it remains unknown whether nonrespondents declined to fill out our questionnaire because of neuropathy symptoms in their hands. In addition, we do not have information on the presence of neuropathy symptoms (eg, idiopathic, entrapment, diabetic, alcoholic or age-related sensory neuropathy) before cancer treatment. However, we were able to control for some conditions that might lead to neuropathy-like symptoms such as diabetes, osteoarthritis, and rheumatoid arthritis. Unfortunately, we were not able to control for multiple sclerosis and fibromyalgia, which could also cause neuropathy-like symptoms. However, the incidence of these conditions is rather low. ${ }^{40,41}$ Furthermore, because many neuropathy symptoms are subjective in nature, assessing them with a self-reported questionnaire is necessary. However, the lack of a clinical-based diagnosis of neuropathy is a limitation of this study. It also is a limitation that information on the exact chemotherapy dosage and the number of cycles given to patients is lacking since dosage seems to influence the severity of neurotoxicity. ${ }^{4}$ Moreover, neuropathy is a dose-limiting adverse effect of oxaliplatin. Therefore, a dose reduction could have taken place in those with acute neuropathy complaints during chemotherapy, which could have had an influence on whether chronic neuropathy occurs later on. Finally, the cross-sectional nature of this study limits the determination of causal associations, for instance, between chemotherapy-induced neuropathy and HRQOL. 
Despite these limitations, the present study provides an important contribution to the limited data available on self-reported neuropathy and its influence on HRQOL. Our results show that, 2 to 11 years after diagnosis, neuropathy symptoms are still reported by a subgroup of patients with CRC. Furthermore, oxaliplatin was associated with sensory symptoms in toes or feet. Finally, this study is one of the first to show that those with many neuropathy symptoms report a lower HRQOL compared to those with less neuropathy symptoms. Because our results are based on a large population-based study with a high response rate, extrapolating these results to the larger population of CRC survivors seems justified.

These results call for further studies on neuropathy and its influence on HRQOL. Preferably, they would be prospective in nature, assess neuropathy objectively and subjectively, and take the dose of oxaliplatin in every cycle and the duration of therapy (cumulative dose) into account. Although many therapies for the prevention of chemotherapy-induced peripheral neuropathy have been investigated, there is no well-accepted proven therapy. ${ }^{42}$ Future studies should therefore focus on possible ways to prevent or alleviate these symptoms, preferably without dose reduction or early cessation of the treatment. With respect to alleviating existing symptoms, results of a randomized placebo-controlled phase III trial showed that duloxetine is an effective treatment for taxane- or platinum-related chemotherapy-induced peripheral neuropathy. ${ }^{43}$ However, if a pharmaceutical approach is of limited benefit, physical therapy and gait and balance training might be helpful in improving physical performance. ${ }^{44}$ 


\section{References}

1. Spiegel R ND, Jemal A.: Cancer statistics 2012. CA Cancer J Clin 2012;62:10-29.

2. kanker S: kanker in Nederland tot 2020; trends en prognoses, 2011

3. Lemmens $\mathrm{V}$, van Steenbergen $\mathrm{L}$, Janssen-Heijnen $\mathrm{M}$, et al: Trends in colorectal cancer in the south of the Netherlands 1975-2007: rectal cancer survival levels with colon cancer survival. Acta Oncologica 2010;49:784-96.

4. Cersosimo RJ: Oxaliplatin-associated neuropathy: a review. Ann Pharmacother 2005;39:128-35.

5. Andre T, Boni C, Mounedji-Boudiaf L, et al: Oxaliplatin, fluorouracil, and leucovorin as adjuvant treatment for colon cancer. N Engl J Med 2004;350:2343-51.

6. Andre T, Boni $\mathrm{C}$, Navarro $\mathrm{M}$, et al: Improved overall survival with oxaliplatin, fluorouracil, and leucovorin as adjuvant treatment in stage II or III colon cancer in the MOSAIC trial. J Clin Oncol 2009;27:3109-16.

7. Beijers AJ, Jongen JL, Vreugdenhil G: Chemotherapy-induced neurotoxicity: the value of neuroprotective strategies. Neth J Med 2012;70:18-25.

8. de Gramont A, Figer A, Seymour M, et al: Leucovorin and fluorouracil with or without oxaliplatin as first-line treatment in advanced colorectal cancer. J Clin Oncol 2000;18:2938-47.

9. Land SR, Kopec JA, Cecchini RS, et al: Neurotoxicity from oxaliplatin combined with weekly bolus fluorouracil and leucovorin as surgical adjuvant chemotherapy for stage II and III colon cancer: NSABP C-07. J Clin Oncol 2007;25:2205-11.

10. Park SB, Lin CS, Krishnan AV, et al: Long-term neuropathy after oxaliplatin treatment: challenging the dictum of reversibility. Oncologist 2011;16:708-16.

11. Hausheer FH, Schilsky RL, Bain S, et al: Diagnosis, management, and evaluation of chemotherapyinduced peripheral neuropathy. Semin Oncol 2006;33:15-49.

12. Postma TJ, Heimans JJ, Muller MJ, et al: Pitfalls in grading severity of chemotherapy-induced peripheral neuropathy. Ann Oncol 1998;9:739-44.

13. Cavaletti G, Frigeni B, Lanzani F, et al: Chemotherapy-Induced Peripheral Neurotoxicity assessment: a critical revision of the currently available tools. Eur J Cancer 2010;46:479-94.

14. van de Poll-Franse LV, Horevoorts N, van Eenbergen M, et al: The Patient Reported Outcomes Following Initial treatment and Long term Evaluation of Survivorship registry: scope, rationale and design of an infrastructure for the study of physical and psychosocial outcomes in cancer survivorship cohorts. Eur J Cancer 2011;47:2188-94.

15. Janssen-Heijnen MLG, Louwman WJ, Van de Poll-Franse LV, et al: Results of 50 years cancer registry in the South of the Netherlands: 1955-2004 (in Dutch). Eindhoven, Eindhoven Cancer Registry, 2005

16. Thong MS, Mols F, Lemmens VE, et al: Impact of chemotherapy on health status and symptom burden of colon cancer survivors: a population-based study. Eur J Cancer 2011;47:1798-807.

17. Thong MS, Mols F, Lemmens VE, et al: Impact of preoperative radiotherapy on general and diseasespecific health status of rectal cancer survivors: a population-based study. Int J Radiat Oncol Biol Phys 2011;81:e49-58.

18. UICC: TNM Atlas Illustrated Guide to the TNM/pTNM Classification of Malignant Tumors, 4th edn, 2nd Revision ed. Berlin, Springer-Verlag, 1992:141-4.

19. Sangha O, Stucki G, Liang $\mathrm{MH}$, et al: The self-administered comorbidity questionnaire: A new method to assess comorbidity for clinical and health services research. Arthritis Rheum 2003;49:156-63.

20. van Duijn C, Keij I: Sociaal-economische status indicator op postcode niveau. Maandstatistiek van de bevolking 2002;50:32-5.

21. Niezgoda HE, Pater JL: A validation study of the domains of the core EORTC quality of life questionnaire. Qual Life Res 1993;2:319-25.

22. Fayers P, Aaronson NK, Bjordal K, et al: The EORTC QLQ-C30 scoring manual (ed 3rd). Brussels, EORTC, 2001

23. Postma TJ, Aaronson NK, Heimans JJ, et al: The development of an EORTC quality of life questionnaire to assess chemotherapy-induced peripheral neuropathy: the QLQ-CIPN20. Eur J Cancer 2005;41: 1135-9. 
24. Babyak MA: What you see may not be what you get: a brief, nontechnical introduction to overfitting in regression-type models. Psychosom Med 2004;66:411-21.

25. Norman GR, Sloan JA, Wyrwich KW: Interpretation of changes in health-related quality of life: the remarkable universality of half a standard deviation. Med Care 2003;41:582-92.

26. Cocks K, King MT, Velikova G, et al: Evidence-based guidelines for determination of sample size and interpretation of the European Organisation for the Research and Treatment of Cancer Quality of Life Questionnaire Core 30. J Clin Oncol 2011;29:89-96.

27. Tofthagen C: Patient perceptions associated with chemotherapy-induced peripheral neuropathy. Clin J Oncol Nurs 2010;14:E22-8.

28. Cavaletti G, Cornblath DR, Merkies IS, et al: The chemotherapy-induced peripheral neuropathy outcome measures standardization study: from consensus to the first validity and reliability findings. Ann Oncol 2013;24(2):454-62.

29. Wolf SL, Barton DL, Qin R, et al: The relationship between numbness, tingling, and shooting/burning pain in patients with chemotherapy-induced peripheral neuropathy (CIPN) as measured by the EORTC QLQ-CIPN20 instrument, N06CA. Support Care Cancer 2012;20:625-32.

30. Tofthagen C, McAllister RD, McMillan SC: Peripheral neuropathy in patients with colorectal cancer receiving oxaliplatin. Clin J Oncol Nurs 2011;15:182-8.

31. Verstappen CC, Heimans JJ, Hoekman K, et al: Neurotoxic complications of chemotherapy in patients with cancer: clinical signs and optimal management. Drugs 2003;63:1549-63.

32. Quasthoff S, Hartung HP: Chemotherapy-induced peripheral neuropathy. J Neurol 2002;249:9-17.

33. Visovsky C: Chemotherapy-induced peripheral neuropathy. Cancer Invest 2003;21:439-51.

34. Soerjomataram I, Thong MS, Ezzati M, et al: Most colorectal cancer survivors live a large proportion of their remaining life in good health. Cancer Causes Control 2012;23:1421-8.

35. Jansen L, Herrmann A, Stegmaier C, et al: Health-related quality of life during the 10 years after diagnosis of colorectal cancer: a population-based study. Journal of clinical oncology : official journal of the American Society of Clinical Oncology 2011;29:3263-9.

36. Tofthagen C: Surviving chemotherapy for colon cancer and living with the consequences. J Palliat Med 2010;13:1389-91.

37. Driessen CM, de Kleine-Bolt KM, Vingerhoets AJ, et al: Assessing the impact of chemotherapy-induced peripheral neurotoxicity on the quality of life of cancer patients : The introduction of a new measure. Support Care Cancer 2012;20:877-81.

38. Kim BJ, Park HR, Roh HJ, et al: Chemotherapy-related polyneuropathy may deteriorate quality of life in patients with B-cell lymphoma. Qual Life Res 2010;19:1097-103.

39. Bakitas MA: Background noise: the experience of chemotherapy-induced peripheral neuropathy. Nurs Res 2007;56:323-31.

40. Kramer MA, van der Maas NA, van Soest EM, et al: Incidence of multiple sclerosis in the general population in the Netherlands, 1996-2008. Neuroepidemiology 2012;39:96-102.

41. Vincent A, Lahr BD, Wolfe F, et al: Prevalence of fibromyalgia: A population-based study in Olmsted County, Minnesota, utilizing the Rochester Epidemiology project. Arthritis Care Res (Hoboken). 2013;65(5):786-92.

42. Pachman DR, Barton DL, Watson JC, et al: Chemotherapy-induced peripheral neuropathy: prevention and treatment. Clin Pharmacol Ther 2011;90:377-87.

43. Smith EML, Pang H, Cirrincione C, et al: CALGB 170601: a phase III double blind trial of duloxetine to treat painful chemotherapyinduced peripheral neuropathy (CPIN). J Clin Oncol (Meeting Abstracts) 2012;30.

44. Wickham R: Chemotherapy-induced peripheral neuropathy: a review and implications for oncology nursing practice. Clin J Oncol Nurs 2007;11:361-76. 


\section{Chapter 4}

Chemotherapy-induced neuropathy in multiple myeloma: influence on quality of life and development of a questionnaire to compose

Common Toxicity Criteria-grading for use in daily clinical practice

Beijers AJ, Vreugdenhil G, Oerlemans S, Eurelings M, Minnema MC, Eeltink CM, van de Poll-Franse LV, Mols F 


\section{Abstract}

Purpose

Chemotherapy-induced peripheral neuropathy (CIPN) may negatively influence multiple myeloma (MM) patients' health-related quality of life (HRQOL). Dose modification is the only way to minimize CIPN. To measure CIPN in daily practice, the Indication for Common Toxicity Criteria (CTC) Grading of Peripheral Neuropathy Questionnaire (ICPNQ) was developed which can be completed within five minutes by the patient. The aim of this study was to (1) perform a psychometric evaluation of the ICPNQ and (2) examine the prevalence of CIPN and its influence on $\mathrm{HRQOL}$ in population-based MM patients.

\section{Methods}

156 MM patients, diagnosed between 2000 and 2014, completed the ICPNQ, EORTC QLQCIPN20 and EORTC QLQ-C30 (65\% response).

\section{Results}

The psychometric analyses showed a Cronbach's alpha of $0.84,0.74$ and 0.61 for respectively the sensory, motoric and autonomic subscale of the ICPNQ. Test-retest reliability and construct validity were good for all subscales. Overall, $65 \%$ of patients reported grade 2-3 neuropathy according to the ICPNQ. Patients with the highest CTC-grades (grade 2 with neuropathic pain and grade $3(38 \%))$ according to the ICPNQ reported significantly worse scores on all EORTC QLQ-CIPN20 subscales compared to patients with lower CTC-grades ( $p \leq 0.002)$. In addition, they reported statistically significant and clinically relevant worse HRQOL scores on almost all EORTC QLQ-C30 subscales.

\section{Conclusions}

CIPN is a common side effect in MM patients, which has a negative impact on HRQOL. The ICPNQ is a valid instrument to distinguish the highest CIPN CTC-grades from the lower CTC-grades necessary to decide on dose modifications of chemotherapy in daily clinical practice. 


\section{Introduction}

Due to improved treatment and increased aging of the population, the prevalence of multiple myeloma ( $\mathrm{MM}$ ) is increasing and more patients are confronted with the side effects of its treatment, which may have a negative influence on patients' healthrelated quality of life (HRQOL). ${ }^{1,2}$ Peripheral neuropathy is a major dose limiting and potentially disabling adverse event of commonly used therapeutic drugs, including immunomodulatory drugs (IMID) thalidomide and lenalidomide, the proteasome inhibitor bortezomib, and vinca alkaloids used in the management of $\mathrm{MM}^{3,4}$ Although those drugs are not all chemotherapy, peripheral neuropathy caused by neurotoxic drugs is often referred to as chemotherapy-induced peripheral neuropathy (CIPN). Since there is no proven management for $\mathrm{CIPN}^{5}$ currently dose modification or discontinuation in case of moderate symptoms of CIPN is the only way to prevent severe CIPN. Unfortunately, dose modifications can influence treatment outcome and should only be applied with caution.

Assessment tools for monitoring CIPN symptoms have been investigated, however none of them were developed for use in clinical practice. ${ }^{6,7}$ Objective CIPN measurements like electromyography and nerve conduction studies are not always patient friendly, time-consuming and expensive as trained personnel is needed. Moreover, it is often not sensitive enough to detect beginning or mild CIPN. ${ }^{6}$ Patientreported questionnaires are recommended for assessing sensory neuropathy. ${ }^{5}$ Nonetheless, guidelines for dose modifications are based on the National Cancer Institute-Common Toxicity Criteria for adverse events (NCI-CTCAE), and existing questionnaires are not developed for this purpose in daily clinical practice. ${ }^{8}$ It remains difficult for the oncologist to interpret which score on a questionnaire responds to which CTC-grade of neuropathy. However, the use of questionnaires would be valuable since the inter- and intra-observer agreement of the $\mathrm{NCl}$-CTC-grading scales have shown to be inadequate, and distinction between grades of CIPN remains difficult for clinicians. ${ }^{9-11}$ Therefore, a valid, quick, and standardized self-reported questionnaire to monitor the CTC-grades of CIPN in patients with MM before and during treatment for use in daily clinical practice is much needed.

A Dutch systematic review concluded that CIPN can have a negative impact on cancer patients' HRQOL. ${ }^{12}$ Nonetheless, no studies among patients with MM were included. A recent German study reported that MM patients commonly experienced sensory neuropathy and that treatment related adverse events and the severity of these symptoms are important determinants of HRQOL among MM patients. ${ }^{1}$ However, the impact of CIPN on the HRQOL of MM patients was not reported.

The objectives of this study are (1) to perform a psychometric evaluation of the selfreported Indication for CTC-Grading of Peripheral Neuropathy questionnaire (ICPNQ) developed for use in daily clinical practice to give a consistent, quick, and accurate 
indication regarding the CTC-grade and (2) to examine the prevalence of CIPN and its influence on $\mathrm{HRQOL}$ in a population-based cohort of MM patients.

\section{Methods}

\section{Participants and data collection}

All patients diagnosed with MM between 2000 and 2014 as registered in the population-based Netherlands Cancer Registry (NCR), location Eindhoven, were eligible for participation. Data collection, which included neuropathy questionnaires and a HRQOL questionnaire, was performed within the PROFILES (Patient Reported Outcomes Following Initial treatment and Long-term Evaluation of Survivorship) registry. ${ }^{13}$ Patients were excluded if they were younger than 18 years of age, had cognitive impairment, had died prior to the study, or had an unverified address. In addition, patients were excluded if they were included in the 2012 PROFILES survey ${ }^{2}$ after which they reported that they did not longer want to participate in the PROFILES survey. A detailed description of the data collection was published earlier. ${ }^{2}$ The participants were asked to complete the questionnaires again within 1 month to establish test-retest reliability.

This study was approved by the certified Medical Ethics Committee of the Máxima Medical Center. All patients gave written informed consent.

\section{Sociodemographic and clinical characteristics}

Sociodemographic and clinical characteristics were available from the NCR and the Population-based HAematological Registry for Observational Studies (PHAROS), ${ }^{14}$ which is an expansion of the NCR. PHAROS aims to provide insight into the current variation, quality and effectiveness of hemato-oncological care in the Netherlands. Information on date of diagnosis, tumour stage and treatment with stem-cell transplantation and/or cytostatics, including type of chemotherapy were collected in PHAROS by trained personnel.

\section{Measurements}

\section{Chemotherapy-induced peripheral neuropathy}

CIPN was assessed with the self-reported ICPNQ (Appendix 4.1), which has been developed by professionals working in hematology and neurology. First, they proposed a list of frequently reported symptoms and the impairments in function they cause. Additionally, existing PRO questionnaires (eg, EORTC QLQ-CIPN20, ${ }^{15}$ Functional 
Assessment of Cancer Therapy/Gynaecology-oncology-Group-neurotoxicity, ${ }^{16}$ Total Neuropathy Score, ${ }^{17}$ and Patient Neurotoxicity Questionnaire ${ }^{18}$ ) were reviewed to select the most important items related to those symptoms and the NCI-CTCAE. By combining these findings the ICPNQ was developed. It contains three hypothesized subscales assessing sensory, autonomic and motor symptoms.

The sensory scale includes five items concerning experienced change in touch, numbness, tingling, change in temperature sensation, and pain. These items can be reported in six different places; toes, feet, lower legs, fingers, hands and lower arms. Patients were instructed to leave the section blank if they had no symptoms. Severity of sensory neuropathy was measured according to the sum of places were symptoms were experienced per item (range 0 to 6). Furthermore, it contains an item 'do you experience the pain intermediately or continuously'.

The autonomic scale contains nine items about experiencing changes in autonomic functions that could be reported by the patient with 'yes'. Patients were instructed to leave the section blank if they had no symptoms. The motoric scale was divided into three items. The first item measured loss of strength, ranging from 'loss of strength in arms or legs' (1) to 'loss of strength in both arms and legs' (2). The subsequent item measured impairment in five instrumental activities, and the third item assessed impairment in six self-care activities. Severity of impairment was measured according to the sum of impairment in instrumental activities (range 0 to 5), and sum of impairments in self-care activities (range 0 to 6 ). If no loss of strength, instrumental or self-care activities were reported, patients were instructed to leave the section blank. Finally, a separate item asked if medical devices were used when walking (no or yes). All subscale sum scores were transformed to a 0-100 scale with higher scores representing more complaints.

After the patient has completed the ICPNQ, an indication regarding the CTCAE-grade can be easily deducted leading to Grade 0: asymptomatic; Grade 1: the presence of sensory symptoms in the absence of motoric and autonomic symptoms; Grade 2: the presence of autonomic symptoms and/or loss of strength, and/or experienced problems in instrumental activities accompanied by sensory symptoms and/or pain; Grade 3: the presence of problems in self-care activities accompanied by sensory symptoms and/or pain and/or loss of strength. Grade 1 and 2 neuropathy can be further divided into categories with or without associated neuropathic pain.

For measuring convergent validity of the ICPNQ, CIPN was also measured with the validated disease-specific EORTC QLQ-CIPN20. ${ }^{15}$ It contains three subscales assessing sensory, motor, and autonomic symptoms. Items are rated on a Likert scale ranging from "not at all" (1) to "very much" (4). Scores were transformed to a 0-100 scale with higher scores representing more complaints. 


\section{Health-related quality of life}

To assess HRQOL, patients completed the Dutch EORTC QLQ-C30 (version 3.0), which has proven to be reliable in $\mathrm{MM}$ patients. ${ }^{19}$ It contains five functional scales on physical, role, cognitive, emotional and social functioning; a global health status/QOL scale; three symptom scales on fatigue, nausea/vomiting and pain; and six single items assessing dyspnea, insomnia, loss of appetite, constipation, diarrhea, and financial impact. Each item is measured on a Likert scale ranging from "not at all" (1) to "very much" (4), except the global health status/QOL scale which ranged from "very poor" (1) to "excellent" (7). Scores were transformed to a 0-100 scale with higher scores on the functioning and global health/QOL scale representing better functioning and QOL, whereas higher scores on the symptom scales represent more complaints.

\section{Hospital anxiety and depression scale and fatigue assessment scale}

For measuring divergent validity of the ICPNQ, the Hospital Anxiety and Depression Scale (HADS) ${ }^{20}$ and the Fatigue assessment scale (FAS) ${ }^{21}$ were used. The HADS is a 14-item scale, dived into two subscales measuring symptoms of anxiety and depression respectively. Items were measured on a 4-point response scale (ranging from 0 , absence of symptoms, to 3, maximum symptomatology). Higher scores represent more symptoms of anxiety and/or depression respectively. Fatigue was assessed with the FAS. It contains ten items. Each item was measured on a 5-point Likert scale ranging from "never" (1) to "always" (5). ${ }^{21}$

\section{Statistical analyses}

All tests were two-sided, considered statistically significant if $p<0.05$, and were performed using IBM SPSS (version 22.0). Sociodemographic and clinical characteristics were compared between patients who completed the test-retest questionnaire and those who did not, using t-test for continuous data and $\mathrm{X}^{2}$ analysis for categorical data. Likewise, sociodemographic characteristics (i.e., sex, age) were compared between respondents and non-respondents.

\section{Psychometric evaluation}

Internal consistency of the ICPNQ subscales was assessed using the Cronbach's alpha coefficient. A value of 0.7 or greater was considered to be adequate. ${ }^{22}$ Test-retest reliability was measured by the intraclass correlation coefficient (ICC), derived from a two-way mixed effect model. Non-parametric Spearman's Rho correlations were assessed for the item-to-own and item-to-other scale reliability. The item-to-own subscale correlation should be higher than the correlation with the other subscales. In 
order to measure convergent validity, Spearman's Rho correlations of the ICPNQ subscales and the related EORTC QLQ-CIPN20 were calculated. EORTC QLQ-CIPN20 subscales were also compared between the CIPN CTC-grades, according to the ICPNQ, used in the dose modification guidelines: grade 0 ; grade 1 without neuropathic pain; grade 1 with neuropathic pain and grade 2 without neuropathic pain combined; and grade 2 with neuropathic pain and grade 3 combined. ${ }^{8,23}$ Subscales were compared between the highest (grade 2 with pain and grade 3 combined) and lower CTC-grades (grade 0 to grade 2 without pain combined), and between the four categories separately with analysis of covariance (ANCOVA). Confounding background variables were determined a priori ${ }^{24}$ and included time since last course of chemotherapy, number of received neurotoxic agents, diabetes mellitus and osteoarthritis. Divergent validity was assessed by calculating correlations between the ICPNQ subscales and the $\mathrm{HADS}^{20}$ and the FAS. ${ }^{21}$ Criteria for quantitative significance of correlations were based on the recommendations of Burnand et al. $^{25}$ : $<0.30$ negligible; $0.30-0.45$ moderate; $0.45-0.60$ substantial; and $>0.60$ high. Known-group validity was examined by comparing the reported ICPNQ CTC-grades according to the modification guidelines in different sociodemographic and clinical subgroups of patients (gender, age, comorbidity, ISS stage, and history of treatment with chemotherapy including number of received neurotoxic drugs and time since last chemotherapy) with $\mathrm{X}^{2}$ analysis.

\section{CIPN and HRQOL among MM patients}

CIPN prevalence was reported as the percentage of patients who reported grades 2 or 3 neuropathy on the ICPNQ. Neuropathy symptoms according to the EORTC QLQCIPN20 that bothered patients the most during the past week were reported with answer categories "quite a bit" and "very much" combined.

To investigate the influence of CIPN on HRQOL, the EORTC QLQ-C30 subscales were compared between patients with the highest (grade 2 with pain and grade 3 combined) and lower CTC-grades (grade 0 to grade 2 without pain combined), using ANCOVA. Confounding background variables were determined a priori ${ }^{24}$ and were chosen to be age, years after since diagnosis, educational level, stage, diabetes mellitus and osteoarthritis. Similar analyses were performed for the patients who reported high ( $4^{\text {th }}$ quartile) vs. low sensory symptoms $\left(1^{\text {st }}-3^{\text {rd }}\right.$ quartile) on the EORTC QLQ-CIPN20. The EORTC QLQ-C30 guidelines were used to determine clinically important difference (CID). ${ }^{26}$ 


\section{Results}

\section{Patient characteristics}

In total, $156 \mathrm{MM}$ patients were included with a response rate of $65 \%$ on the inquiry. Respondents were significantly younger at time of diagnosis (64.0 vs. $67.7, p=0.004$ ), and were more often male $(71 \%$ vs $47 \%, p<0.001)$ compared to the non-respondents. Of the total group of respondents, patients were more often male $(n=110,71 \%)$, on average 67.5 years of age at time of the survey, and on average 3.3 years (SD 2.4, range 1-13 years) since diagnosis (Table 4.1). Patients were most often diagnosed with ISS stage I or II disease (35\% and $27 \%$ respectively), and the majority was treated with chemotherapy (92\%), mostly with a regimen including bortezomib or thalidomide. Stem cell transplantation was received by 64 patients (41\%). Patients who completed the test-retest questionnaire $(n=117,75 \%)$ were diagnosed less recently compared to patients who did not complete the test-retest $(n=39)$ (Table 4.1).

Table 4.1 Baseline sociodemographic and clinical characteristics of multiple myeloma patients ( $N=156)$, and differences between patients who completed the test-retest $(\mathrm{N}=117)$ and who did not complete test-retest ( $\mathrm{N}=39$ ).

\begin{tabular}{|c|c|c|c|c|}
\hline & $\begin{array}{l}\text { All } \mathrm{MM} \text { patients } \\
\mathrm{N}=156\end{array}$ & $\begin{array}{c}\text { Test-retest } \\
\mathrm{N}=117 \\
\end{array}$ & $\begin{array}{c}\text { No Test-retest } \\
\qquad \mathrm{N}=39 \\
\end{array}$ & p-value* \\
\hline \multicolumn{5}{|c|}{ Age at time of survey, years } \\
\hline Mean (SD) & $67.5(9.3)$ & $67.7(9.0)$ & $67.0(10.3)$ & 0.66 \\
\hline \multicolumn{5}{|c|}{ Years since diagnosis } \\
\hline Mean (SD) & $3.3(2.4)$ & $3.5(2.6)$ & $2.6(1.4)$ & 0.005 \\
\hline \multicolumn{5}{|c|}{ Age at time of diagnosis, years $(\mathrm{N}, \%)$} \\
\hline$<65$ years & $84(54)$ & $64(55)$ & $20(51)$ & 0.71 \\
\hline$>65$ years & $72(46)$ & $53(45)$ & $19(49)$ & \\
\hline \multicolumn{5}{|l|}{ Gender } \\
\hline Male & $110(71)$ & $84(71)$ & $26(67)$ & 0.54 \\
\hline Female & $46(30)$ & $33(28)$ & $13(33)$ & \\
\hline \multicolumn{5}{|c|}{ Educational level (N, \%) } \\
\hline Low & 29 (19) & $11(9)$ & $9(23)$ & 0.06 \\
\hline Medium & $92(59)$ & $73(62)$ & $24(62)$ & \\
\hline High & $32(21)$ & $31(26)$ & $6(15)$ & \\
\hline \multicolumn{5}{|c|}{ Clinical Data at cancer diagnosis } \\
\hline \multicolumn{5}{|c|}{ Stage ISS (N, \%) } \\
\hline 1 & $54(35)$ & $45(38)$ & $9(23)$ & 0.38 \\
\hline II & $42(27)$ & $30(26)$ & $12(31)$ & \\
\hline III & 27 (17) & $19(16)$ & $8(21)$ & \\
\hline Unknown & $33(21)$ & $23(20)$ & $10(26)$ & \\
\hline
\end{tabular}


Table $4.1 \quad$ (continued)

\begin{tabular}{|c|c|c|c|c|}
\hline & $\begin{array}{l}\text { All MM patients } \\
\qquad \mathrm{N}=\mathbf{1 5 6}\end{array}$ & $\begin{array}{c}\text { Test-retest } \\
\mathrm{N}=117\end{array}$ & $\begin{array}{l}\text { No Test-retest } \\
\qquad \mathrm{N}=39\end{array}$ & p-value* \\
\hline \multicolumn{5}{|c|}{$\begin{array}{l}\text { Physical performance at completion } \\
\text { questionnaire** }\end{array}$} \\
\hline High & $32(21)$ & $28(24)$ & $4(10)$ & 0.13 \\
\hline Medium & $92(59)$ & $69(59)$ & $23(59)$ & \\
\hline Low & 29 (19) & $19(16)$ & $10(26)$ & \\
\hline Unknown & $3(2)$ & $1(1)$ & $2(5)$ & \\
\hline \multicolumn{5}{|c|}{$\begin{array}{l}\text { Treatment received during whole } \\
\text { disease trajectory }\end{array}$} \\
\hline \multicolumn{5}{|c|}{ Stem cell transplant $(\mathrm{N}, \%)$} \\
\hline Yes & $64(41)$ & $49(42)$ & $15(38)$ & 0.68 \\
\hline No & $91(58)$ & $67(57)$ & $24(61)$ & \\
\hline Unknown & $1(1)$ & $1(1)$ & $0(0)$ & \\
\hline \multicolumn{5}{|c|}{ Chemotherapy treatment $(\mathrm{N}, \%)$} \\
\hline Yes & $143(92)$ & $108(92)$ & $35(90)$ & 0.50 \\
\hline No & $12(8)$ & $8(7)$ & $4(10)$ & \\
\hline Unknown & $1(1)$ & $1(1)$ & $0(0)$ & \\
\hline \multicolumn{5}{|c|}{ Received neurotoxic drug ( $\mathrm{N}, \%)$} \\
\hline Thalidomide & $73(47)$ & $52(44)$ & $21(54)$ & 0.33 \\
\hline Bortezomib & $80(51)$ & $58(50)$ & $22(56)$ & 0.49 \\
\hline Lenalidomide & $60(39)$ & $48(41)$ & $12(31)$ & 0.24 \\
\hline Vincristine & $9(6)$ & $9(8)$ & $0(0)$ & 0.07 \\
\hline \multicolumn{5}{|c|}{$\begin{array}{l}\text { No. of comorbid conditions at time of } \\
\text { survey }(N, \%)\end{array}$} \\
\hline None & $26(17)$ & $20(17)$ & $6(15)$ & 0.79 \\
\hline 1 & $33(21)$ & $25(21)$ & $8(21)$ & \\
\hline $2+$ & $59(38)$ & $48(41)$ & $11(28)$ & \\
\hline Unknown & $38(24)$ & $24(21)$ & $14(35)$ & \\
\hline \multicolumn{5}{|c|}{ Most frequent conditions ( $\mathrm{N}, \%)$} \\
\hline Back pain & $65(42)$ & $54(46)$ & $11(28)$ & 0.23 \\
\hline Osteoartritis & $49(31)$ & $38(32)$ & $11(28)$ & 0.99 \\
\hline Hypertension & $41(26)$ & $33(28)$ & $8(21)$ & 0.77 \\
\hline Anemia & $28(18)$ & $20(17)$ & $8(21)$ & 0.44 \\
\hline Heart disease & 29 (19) & $22(19)$ & $7(18)$ & 0.66 \\
\hline Pulmonary disease & $16(10)$ & $13(11)$ & $3(8)$ & 0.73 \\
\hline Diabetes Mellitus & $11(7)$ & $6(5)$ & $5(13)$ & 0.06 \\
\hline
\end{tabular}

Due to rounding off percentages can exceed $100 \%$. $^{*}$ P-value represents differences in sociodemographic and clinical characteristics between patients who completed the test-retest $(n=117)$ and patients who did not complete the test-retest $(n=39)$. ${ }^{* *}$ Physical Performance: physical performance scale of the EORTC QLQ-C30 divided into 3 categories based on average and standard deviations derived from van de Poel et al., $2014^{31}: 1$ SD under average physical functioning of MM patients is considered low physical functioning, whereas 1 SD above average MM patient physical functioning is considered high physical functioning.

\section{Psychometric evaluation}

The internal consistencies of the ICPNQ sensory and motoric subscales were adequate with a Cronbach's alpha of 0.84 and 0.70 , respectively (Table 4.2). After deletion of 
the question 'do you use medical devices when walking' from the motoric subscale, a higher Cronbach's alpha was observed, 0.74 . The Cronbach's alpha of the autonomic scale was moderate with 0.61 . All items correlated best with their own subscale. Testretest reliability of the subscales was good with ICC of 0.83 (Cl 95\% 0.76-0.89), 0.83 (Cl 95\% 0.75-0.89) and $0.77(\mathrm{Cl} 95 \% 0.67-0.84)$ for the sensory, motoric and autonomic scales, respectively. Test-retest for the defined ICPNQ grades was 0.67 (Cl 95\% 0.53-0.77).

Table 4.2 Internal consistency; item-to-own and item-to-other scale correlations*; test-retest reliability with intraclass correlation coefficient $\left(\right.$ ICC**) $^{* *}$ of the ICPNQ.

\begin{tabular}{lccccc}
\hline $\begin{array}{l}\text { Questionnaire } \\
\text { subscales }\end{array}$ & $\begin{array}{c}\text { Number of } \\
\text { Items }\end{array}$ & $\begin{array}{c}\text { Cronbach's } \\
\text { Alpha }\end{array}$ & $\begin{array}{c}\text { Item-to-own- } \\
\text { scale }\end{array}$ & $\begin{array}{c}\text { Item-to-other } \\
\text { scale }\end{array}$ & $\begin{array}{c}\text { Test-retest ICC** } \\
\mathbf{( 9 5 \% ~ C l )}\end{array}$ \\
\hline Sensory & 6 & 0.84 & $0.55-0.68$ & $0.19-0.48$ & $0.83(0.76-0.89)$ \\
Motor & 3 & 0.74 & $0.77-0.78$ & $0.20-0.44$ & $0.83(0.75-0.89)$ \\
Autonomic & 9 & 0.61 & $0.17-0.55$ & $0.01-0.28$ & $0.77(0.67-0.84)$ \\
\hline
\end{tabular}

ICC: intraclass correlation coefficient; ICPNQ: Indication for Common Toxicity Criteria grading of Peripheral Neuropathy Questionnaire. * Correlations calculated with Spearman's Rho. ** Test-retest intraclass correlation coefficient, calculated with two-way mixed model.

All subscales correlated best with their related EORTC QLQ-CIPN20 subscales with a substantial to high correlation, indicating good convergent validity (Table 4.3). Divergent validity showed that depression and fatigue, as assessed with the HADS and FAS, was substantially correlated with motoric functioning $(r=0.49$ and $r=0.47$, $p<0.001)$. Sensory and autonomic functions were moderately correlated with depression $(r=0.35$ and $r=0.33, p<0.001)$ and fatigue $(r=0.35$ and $r=0.32, p<0.001)$ respectively.

Table 4.3 Convergent and divergent validity of the ICPNQ with the EORTC QLQ-CIPN20, and the HADS and FAS, respectively.

\begin{tabular}{lcccccc}
\hline ICPNQ & \multicolumn{3}{c}{ Convergent validity } & \multicolumn{2}{c}{ Divergent validity } \\
\cline { 2 - 7 } & \multicolumn{3}{c}{ EORTC QLQ-CIPN20 } & \multicolumn{2}{c}{ HADS } & FAS \\
\cline { 2 - 6 } & Sensory & Motor & Autonomic & Anxiety & Depression & Total \\
\hline Sensory & $0.72^{*}$ & $0.51^{*}$ & $0.23^{*}$ & $0.23^{*}$ & $0.35^{*}$ & $0.35^{*}$ \\
Motor & $0.42^{*}$ & $0.58^{*}$ & $0.40^{*}$ & $0.36^{*}$ & $0.49^{*}$ & $0.47^{*}$ \\
Autonomic & $0.29^{*}$ & $0.31^{*}$ & $0.40^{*}$ & $0.27^{*}$ & $0.33^{*}$ & $0.32^{*}$ \\
\hline
\end{tabular}

EORTC QLQ-CIPN20; European Organisation for Research and Treatment of Cancer Quality of Life Questionnaire Chemotherapy-Induced Peripheral Neuropathy 20; FAS: Fatigue Assessment Scale; HADS: Hospital Anxiety and Depression Scale; ICPNQ: Indication for Common Toxicity Criteria grading of Peripheral Neuropathy Questionnaire. * correlation ( $r$ ) calculated with Spearman's Rho with $p$ value $<0.001$. 
Patients with the highest CTCAE-grades $(n=59)$, according to the ICPNQ, reported significantly worse mean scores on all the EORTC QLQ-CIPN20 subscales compared to patients with lower CTCAE-grades ( $n=97$ ) (all $p \leq 0.002$ ). In addition, they reported significantly worse mean scores on the sensory and motoric EORTC QLQ-CIPN20 subscales than patients with grade 1 with and grade 2 without neuropathic pain combined $(n=53)$ (all $p \leq 0.01$ ) (Figure 4.1). Within the categories of patients with lower CTCAE-grades, the average scores of patients with grade 0 neuropathy on sensory (average 2.1 vs. 16.0, $\mathrm{p}=0.405$ ) and motoric (4.4 vs. 7.5, $\mathrm{p}=1.0$ ) EORTC QLQ-CIPN20 subscales were lower compared to patients with grade 1 without pain, although not statistically significant. In addition, the average scores on sensory (2.1 vs. 13.7, $p=0.398$ ) and motoric ( 4.4 vs. $13.2 p=0.347$ ) subscales were also lower compared to those with grade 1 with pain and grade 2 without pain combined.

Known-group validity showed that the ICPNQ was able to distinguish patients who received treatment with chemotherapy and who had a lower physical performance status (Supplementary Table S4.1). No differences between CTC-grades were observed for other subgroups.

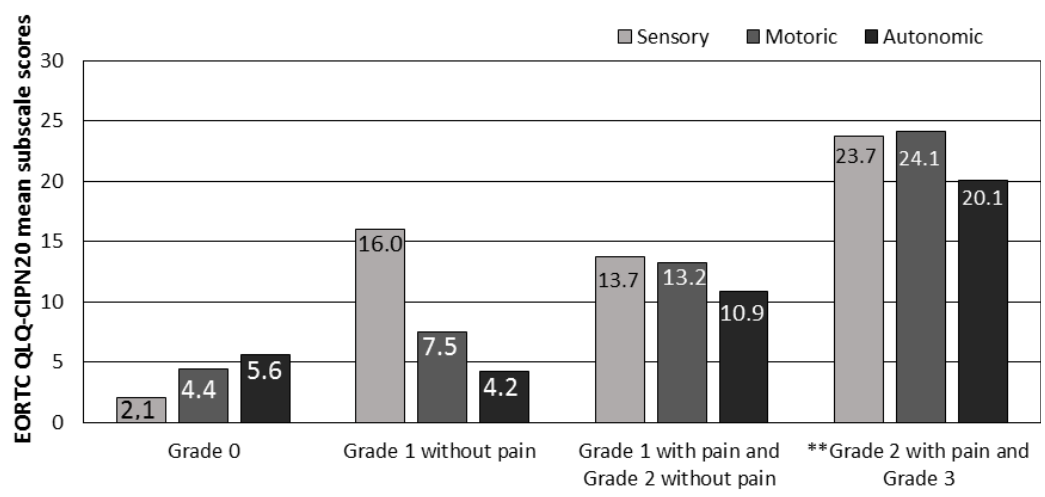

*ICPNQ CTC-categories according to dose modification guidelines

Figure 4.1 Distribution of EORTC QLQ-CIPN20 mean subscale scores divided by categories of Common Toxicity Criteria Grade according to the ICPNQ.

CIPN: Chemotherapy-induced peripheral neuropathy; CTC: Common Toxicity Criteria; EORTC QLQ-CIPN20: European Organisation for Research and Treatment of Cancer Quality of Life Questionnaire Chemotherapy-Induced Peripheral Neuropathy 20; ICPNQ: Indication for Common Toxicity Criteria grading of Peripheral Neuropathy Questionnaire. * Categories of CTC-grade corresponding to the dose modification guidelines ${ }^{8}$ : grade 0 ; grade 1 without pain; grade 1 with pain and grade 2 without pain combined; grade 2 with pain and grade 3 combined. ${ }^{* *} \mathrm{P} \leq 0.001$ : Patients with the highest CTC-grades (grade 2 with pain and grade 3 ) according to the ICPNQ reported significantly worse mean scores on all EORTC QLQ-CIPN20 subscales compared to patients with lower CTC-grades combined. Standard deviations: Sensory scale: 2.8, 10.3, 14.5, 19.4 respectively; Motoric scale: 8.0, 7.4, 15.2, 20.9 respectively; Autonomic scale: $10.3,7.5,16.1,22.2$ respectively. 


\section{CIPN and HRQOL among MM patients}

Overall, $65 \%$ of patients reported CTCAE-grades 2 or 3 neuropathy according to the ICPNQ (Figure 4.2). The reported neuropathy symptoms were mainly sensory and consisted of tingling in lower and/or upper extremities (37\%), pain (burning, stabbing, stinging or cramping) (37\%), and numbness in lower and/or upper extremities (27\%). Loss of strength in upper and/or lower extremities (22\%), and the autonomic symptoms problem achieving an erection in male/ trouble with lubrication in women (28\%) and more sweating (25\%) were often reported (Supplementary Table S4.2). According to the EORTC QLQ-CIPN20, 53\% of patients reported at least one symptom that bothered them "quite a bit" or "very much", and they reported similar symptoms as the ICPNQ (data not shown).

\section{ICPNQ: CTC-grades in percentage}

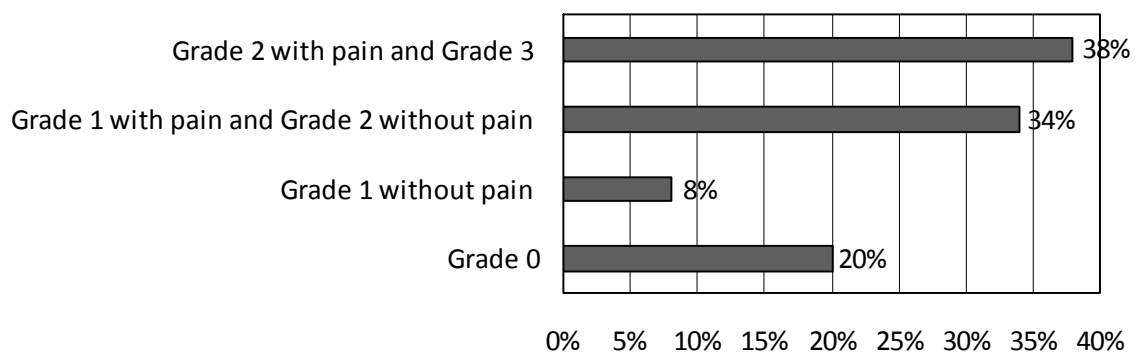

Figure 4.2 Percentage of patients with $\mathrm{MM}$ that reported different categories of Common Toxicity Criteria Grade according to the ICPNQ ( $N=156)$. CTC: Common Toxicity Criteria; ICPNQ: Indication for Common Toxicity Criteria grading of Peripheral Neuropathy Questionnaire; MM: Multiple Myeloma.

Patients who reported the highest CTC-grades according to the ICPNQ reported statistically significant and clinically relevant worse scores on all of the functioning scales and global health status/QOL scale of the EORTC QLQ-C30 compared to patients with lower CTC-grades. They also reported significantly worse scores on the symptom scales fatigue, pain, dyspnea, insomnia, and loss of appetite. These differences were also clinically important to patients, varying from large to small clinical relevance (Table 4.4). Comparison of the EORTC QLQ-C30 subscales between patients who reported the highest ( $4^{\text {th }}$ quartile) sensory subscale according to the EORTC QLQCIPN20 and patient with a lower $\left(1^{\text {st }}-3^{\text {rd }}\right.$ quartile $)$ sensory subscale showed similar results (data not shown). 
Table 4.4 Comparison of the EORTC QLQ-C30 subscale scores among MM patients with highest grades of neuropathy vs. lowest grades of neuropathy according to the ICPNQ ( $N=156)$.

\begin{tabular}{|c|c|c|c|c|}
\hline \multirow[t]{2}{*}{ Subscales } & \multicolumn{2}{|c|}{ ICPNQ } & \multirow[t]{2}{*}{ P-value $^{c}$} & \multirow[t]{2}{*}{$\operatorname{CID}^{d}$} \\
\hline & $\begin{array}{c}\text { Lower grades }^{\mathrm{a}} \\
\text { Mean (SD) } \\
\end{array}$ & $\begin{array}{c}\text { Highest grades }^{\mathrm{b}} \\
\text { Mean (SD) }\end{array}$ & & \\
\hline \multicolumn{5}{|l|}{ EORTC QLQ-C30 } \\
\hline Global health status/QOL & $72.8(19.6)$ & $57.2(23.5)$ & $<0.001$ & Large \\
\hline Physical functioning & $76.9(17.5)$ & $61.0(21.2)$ & $<0.001$ & Medium \\
\hline Role Functioning & $74.8(25.3)$ & $54.2(30.6)$ & $<0.001$ & Medium \\
\hline Emotional functioning & $82.6(22.8)$ & $72.7(22.5)$ & 0.009 & Small \\
\hline Cognitive functioning & $85.6(18.1)$ & $68.7(26.7)$ & $<0.001$ & Large \\
\hline Social functioning & $83.2(23.0)$ & $67.3(28.5)$ & $<0.001$ & Large \\
\hline Fatigue & $25.8(22.4)$ & $47.7(24.6)$ & $<0.001$ & Large \\
\hline Nausea and vomiting & $4.4(12.0)$ & $7.3(14.6)$ & 0.22 & - \\
\hline Pain & $22.0(26.3)$ & $43.5(27.7)$ & $<0.001$ & Large \\
\hline Dyspnea & $15.8(24.4)$ & $33.3(28.4)$ & $<0.001$ & Large \\
\hline Insomnia & $19.9(26.9)$ & $33.3(31.6)$ & 0.04 & Medium \\
\hline Loss of appetite & $6.5(17.2)$ & $17.5(25.8)$ & $<0.001$ & Small \\
\hline Constipation & $8.2(16.8)$ & $17.5(27.5)$ & 0.07 & - \\
\hline Diarrhea & 7.5 (16.4) & $12.3(19.6)$ & 0.07 & - \\
\hline Financial impact & $12.2(22.9)$ & $21.6(34.2)$ & 0.12 & - \\
\hline
\end{tabular}

EORTC QLQ-C30: European Organisation for Research and Treatment of Cancer Quality of Life Questionnaire; ICPNQ: Indication for Common Toxicity Criteria grading of Peripheral Neuropathy Questionnaire. ${ }^{a}$ Lowest grades; patients with grade 0 to 2 without pain according to the ICPNQ. b Highest

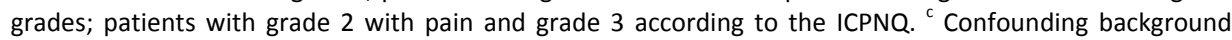
variables included for adjustment were chosen a priori and were age at time of questionnaire, years since diagnosis, educational level, stage, diabetes mellitus, osteoarthritis. ${ }^{d}$ CID: clinically important difference, based on guidelines of EORTC QLQ-C30. ${ }^{26}$

\section{Discussion}

This study showed that the self-reported ICPNQ is a valid instrument to distinguish the higher CIPN CTC-grades from the lower CTC-grades in a population-based cohort of MM patients. After instruction, most patients are able to complete the questionnaire within five minutes. Therefore, this self-reported questionnaire may help to give an indication of the neuropathy CTCAE-grade in a uniform way and may prove to be of value in guiding the dose modifications during treatment in daily clinical practice. Since clinicians tend to underreport subjective toxicities, the use of patient-reported outcome measurements is highly recommended in the assessment of adverse events. ${ }^{9,11}$ Dose modification guidelines are based on the $\mathrm{NCl}-\mathrm{CTCAE}^{8}$ and the current patient-reported CIPN questionnaires, not based on NCI-CTCAE nor developed for the use in individual patients, are difficult to use in daily clinical practice. Guidelines recommend a dose reduction of bortezomib in MM patients with either grade 1 with 
or grade 2 without neuropathic pain, and cessation of bortezomib in the case of grade 2 with neuropathic pain or grade $3 \mathrm{CIPN}^{8,23}$ However, distinction between those grades of CIPN remains difficult for clinicians. ${ }^{10}$ The current study confirms that the ICPNQ is able to distinguish these two groups of CTCAE-grades. Therefore, the selfreported ICPNQ provides in the need to give a consistent indication regarding the CTCAE-grades of CIPN, overcomes the issue of underreporting CIPN by clinicians, and may be incorporated easily in clinical practice in the decision on applying dose modifications. To evaluate the ability to monitor the development of CIPN over time, and to compare the self-reported CTC-grades with physician reported CTC-grades, and its use in decisions regarding dose reductions, further prospective evaluation is needed.

CIPN was reported in more than half of MM patients up to 13 years after diagnosis and it has a negative influence on patients' HRQOL. Since overall treatment outcome among MM patients has been improved, toxicity and HRQOL during and after MM treatment is becoming more important and should be incorporated more often in clinical decision-making. Only few studies investigated the influence of CIPN on HRQOL by use of a self-reported CIPN measurement in MM patients. They also reported that the severity of side effects, including CIPN, was related to worse HRQOL. ${ }^{27-29}$ Therefore, prevention of CIPN is warranted and a self-reported measurement instrument which is useful in the decision on applying an early dose modification in the case of moderate CIPN symptoms is therefore much needed.

The strengths of this study are a population-based sample of MM patients with a good response rate to the inquiry. Therefore, it seems justified to extrapolate these results to the overall MM population. However, the study also has some limitations. Although the response rate was good, it remains unknown whether non-respondents did not fill in the questionnaire because of neuropathy symptoms (to the hands), poor health, or absence of symptoms. In addition, respondents were more often male which may have influenced the results. We did not have information on pre-existing neuropathy before chemotherapy treatment, and therefore, a causal relation between received treatment and neuropathy cannot be given. We were able to adjust for osteoarthritis and diabetes mellitus, which causes neuropathy-like symptoms. Patients were instructed to leave the section blank if they had no symptoms. Therefore patients who did not report any neuropathy symptoms on the ICPNQ were assumed to be asymptomatic instead of missing. This seemed justified, as they also scored significantly lower on the EORTC QLQ-CIPN20 compared to patients who did report symptoms on the ICPNQ. In addition, somewhat more patients answered the question on whether the pain was 'continuous or intermittently present' compared to patients who reported a positive answer on the question about 'pain'. Therefore, further research on acceptability of this part of the question has to be done. Furthermore, convergent and construct validity of the ICPNQ were good for the sensory and the motoric subscales. The autonomic subscale had an internal consistency of 0.61 , which 
is below the cut-off value of 0.7 indicating adequate internal consistency. Autonomic dysfunction is difficult to measure, and the autonomic items of the EORTC QLQCIPN20 also showed to have low item-item correlation. ${ }^{30}$ Yet, the autonomic subscale of the ICPNQ correlated substantially with the EORTC QLQ-CIPN20 autonomic subscale. Moreover, patients were up to 13 years after diagnosis, although some patients were still actively treated for MM. This may have influenced CIPN and HRQOL, but we were able to control our analysis for time since last course of chemotherapy. Furthermore, the cross-sectional design of our study impedes drawing firm conclusions.

In conclusion, CIPN is a common side effect, which is reported in more than half of patients with MM. Since there is no proven treatment for CIPN, dose modification in case of moderate symptoms is the only way to prevent severe CIPN. The self-reported ICPNQ is a valid instrument to distinguish the highest CIPN CTC-grades from the lower CTC-grades necessary for the decision on applying dose modifications of chemotherapy during treatment in patients with MM. Therefore, the ICPNQ provides in the need to give a standardized indication regarding the CTC-grades of CIPN, overcomes the issue of underreporting CIPN by clinicians, and can be incorporated easily in clinical practice. Patients with grade 2 with pain and grade 3 neuropathy combined reported statistically significant and clinically relevant worse HRQOL compared to patients who had neuropathy CTC-grades between 0 to 2 without pain. This indicates that early dose modification based on a more reliable tool for CIPN measurements is needed. Further prospective evaluation of the ICPNQ is needed before it can be used in daily practice. This prospective evaluation should incorporate pre-existing neuropathy and may prove to be of value in monitoring progression of CIPN and guiding dose modification decisions. It may show whether the assessment is leading to both the ability to keep patients on treatment and to reduce the symptoms over time in patients with MM.

\section{Acknowledgement}

The study was supported by Janssen-Cilag pharmaceutical companies of JohnsonJohnson. 


\section{References}

1. Jordan K, Proskorovsky I, Lewis P, Ishak J, Payne K, Lordan N et al. Effect of general symptom level, specific adverse events, treatment patterns, and patient characteristics on health-related quality of life in patients with multiple myeloma: results of a European, multicenter cohort study. Support Care Cancer 2014;22:417-26.

2. Mols F, Oerlemans S, Vos AH, Koster A, Verelst S, Sonneveld P et al. Health-related quality of life and disease-specific complaints among multiple myeloma patients up to $10 \mathrm{yr}$ after diagnosis: results from a population-based study using the PROFILES registry. Eur J Haematol 2012;89:311-9.

3. Delforge M, Blade J, Dimopoulos MA, Facon T, Kropff M, Ludwig $\mathrm{H}$ et al. Treatment-related peripheral neuropathy in multiple myeloma: the challenge continues. Lancet Oncol 2010;11:1086-95.

4. Jongen JL, Broijl A, Sonneveld P. Chemotherapy-induced peripheral neuropathies in hematological malignancies. J Neurooncol 2015;121:229-37.

5. Hershman DL, Lacchetti C, Dworkin RH, Lavoie Smith EM, Bleeker J, Cavaletti G et al. Prevention and Management of Chemotherapy-Induced Peripheral Neuropathy in Survivors of Adult Cancers: American Society of Clinical Oncology Clinical Practice Guideline. J Clin Oncol 2014;32:1941-67.

6. Cavaletti G, Frigeni B, Lanzani F, Mattavelli L, Susani E, Alberti P et al. Chemotherapy-Induced Peripheral Neurotoxicity assessment: a critical revision of the currently available tools. Eur J Cancer 2010;46:479-94.

7. Cavaletti G, Cornblath DR, Merkies IS, Postma TJ, Rossi E, Frigeni B et al. The chemotherapy-induced peripheral neuropathy outcome measures standardization study: from consensus to the first validity and reliability findings. Ann Oncol 2013;24:454-62.

8. Zweegman S, Lokhorst HM, Levin MD, Waal de E, Bos GMJ, Kersten MJ et al. Guidelines for the treatment of multiple myeloma 2012. The Dutch Journal of Hematology 2012;8:300-20.

9. Di Maio M, Gallo C, Leighl NB, Piccirillo MC, Daniele G, Nuzzo F et al. Symptomatic toxicities experienced during anticancer treatment: agreement between patient and physician reporting in three randomized trials. J Clin Oncol 2015;33:910-5.

10. Postma TJ, Heimans JJ, Muller MJ, Ossenkoppele GJ, Vermorken JB, Aaronson NK. Pitfalls in grading severity of chemotherapy-induced peripheral neuropathy. Ann Oncol 1998;9:739-44.

11. Basch E, lasonos A, McDonough T, Barz A, Culkin A, Kris MG et al. Patient versus clinician symptom reporting using the National Cancer Institute Common Terminology Criteria for Adverse Events: results of a questionnaire-based study. Lancet Oncol 2006;7:903-9.

12. Mols F, Beijers T, Vreugdenhil G, van de Poll-Franse L. Chemotherapy-induced peripheral neuropathy and its association with quality of life: a systematic review. Support Care Cancer 2014;22:2261-9.

13. van de Poll-Franse LV, Horevoorts N, van Eenbergen M, Denollet J, Roukema JA, Aaronson NK et al. The Patient Reported Outcomes Following Initial treatment and Long term Evaluation of Survivorship registry: scope, rationale and design of an infrastructure for the study of physical and psychosocial outcomes in cancer survivorship cohorts. Eur J Cancer 2011;47:2188-94.

14. PHAROS. Population-based Haematological Registry for Observational Studies (PHAROS), http://www.pharosregistry.nl/. Accessed 1 June 2015

15. Postma TJ, Aaronson NK, Heimans JJ, Muller MJ, Hildebrand JG, Delattre JY et al. The development of an EORTC quality of life questionnaire to assess chemotherapy-induced peripheral neuropathy: the QLQ-CIPN20. Eur J Cancer 2005;41:1135-9.

16. Calhoun EA, Welshman EE, Chang CH, Lurain JR, Fishman DA, Hunt TL et al. Psychometric evaluation of the Functional Assessment of Cancer Therapy/Gynecologic Oncology Group-Neurotoxicity (Fact/GOG-Ntx) questionnaire for patients receiving systemic chemotherapy. Int J Gynecol Cancer 2003;13:741-8.

17. Cavaletti G, Bogliun G, Marzorati L, Zincone A, Piatti M, Colombo N et al. Grading of chemotherapyinduced peripheral neurotoxicity using the Total Neuropathy Scale. Neurology 2003;61:1297-300.

18. Hausheer FH, Schilsky RL, Bain S, Berghorn EJ, Lieberman F. Diagnosis, management, and evaluation of chemotherapy-induced peripheral neuropathy. Semin Oncol 2006;33:15-49. 
19. Wisloff F, Eika S, Hippe E, Hjorth M, Holmberg E, Kaasa S et al. Measurement of health-related quality of life in multiple myeloma. Nordic Myeloma Study Group. Br J Haematol 1996;92:604-13.

20. Zigmond AS, Snaith RP. The hospital anxiety and depression scale. Acta Psychiatr Scand 1983;67: 361-70.

21. Michielsen HJ, De Vries J, Van Heck GL. Psychometric qualities of a brief self-rated fatigue measure: The Fatigue Assessment Scale. J Psychosom Res 2003;54:345-52.

22. Nunnally JC. Psychometric theory. New York: McGraw-Hill 1978.

23. Richardson PG, Delforge M, Beksac M, Wen $P$, Jongen JL, Sezer $O$ et al. Management of treatmentemergent peripheral neuropathy in multiple myeloma. Leukemia 2012;26:595-608.

24. Babyak MA. What you see may not be what you get: a brief, nontechnical introduction to overfitting in regression-type models. Psychosom Med 2004;66:411-21.

25. Burnand B, Kernan WN, Feinstein AR. Indexes and boundaries for "quantitative significance" in statistical decisions. J Clin Epidemiol 1990;43:1273-84.

26. Cocks K, King MT, Velikova G, Martyn St-James M, Fayers PM, Brown JM. Evidence-based guidelines for determination of sample size and interpretation of the European Organisation for the Research and Treatment of Cancer Quality of Life Questionnaire Core 30. J Clin Oncol 2011;29:89-96.

27. Lee SJ, Richardson PG, Sonneveld P, Schuster MW, Irwin D, San Miguel JF et al. Bortezomib is associated with better health-related quality of life than high-dose dexamethasone in patients with relapsed multiple myeloma: results from the APEX study. Br J Haematol 2008;143:511-9.

28. Dubois D, Dhawan R, van de Velde H, Esseltine D, Gupta S, Viala M et al. Descriptive and prognostic value of patient-reported outcomes: the bortezomib experience in relapsed and refractory multiple myeloma. J Clin Oncol 2006;24:976-82.

29. Boland E, Eiser C, Ezaydi Y, Greenfield DM, Ahmedzai SH, Snowden JA. Living with advanced but stable multiple myeloma: a study of the symptom burden and cumulative effects of disease and intensive (hematopoietic stem cell transplant-based) treatment on health-related quality of life. J Pain Symptom Manage 2013;46:671-80.

30. Lavoie Smith EM, Barton DL, Qin R, Steen PD, Aaronson NK, Loprinzi CL. Assessing patient-reported peripheral neuropathy: the reliability and validity of the European Organization for Research and Treatment of Cancer QLQ-CIPN20 Questionnaire. Qual Life Res 2013;22:2787-99.

31. van der Poel MW, Oerlemans S, Schouten HC, van de Poll-Franse LV. Elderly multiple myeloma patients experience less deterioration in health-related quality of life than younger patients compared to a normative population: a study from the population-based PROFILES registry. Ann Hematol 2015;94:651-61. 


\section{Appendix 4.1}

Table S4.1 Know-group validity of the ICPNQ*, $\mathrm{n}=156$.

\begin{tabular}{|c|c|c|c|c|c|}
\hline & $\begin{array}{l}n=31 \\
n(\%)\end{array}$ & $\begin{array}{c}\text { grade } 1 \\
\text { without pain }\end{array}$ & $\begin{array}{c}\text { grade } 1 \text { with pain } \\
\text { and grade } 2 \\
\text { without pain } \\
\text { combined } \\
n=53 \\
n(\%)\end{array}$ & $\begin{array}{l}\text { grade } 2 \text { with pain } \\
\text { and grade } 3 \\
\text { combined } \\
\\
n=59 \\
n(\%)\end{array}$ & p-value** \\
\hline \multicolumn{6}{|l|}{ Gender } \\
\hline Male & $23(74)$ & $9(69)$ & $40(76)$ & $38(64)$ & 0.59 \\
\hline Female & $8(26)$ & $4(31)$ & $13(25)$ & $21(36)$ & \\
\hline \multicolumn{6}{|l|}{ Age at time of } \\
\hline$<65$ years & $11(36)$ & $6(46)$ & $24(45)$ & $18(31)$ & \\
\hline$>65$ years & $20(65)$ & $7(54)$ & $29(55)$ & $41(70)$ & \\
\hline \multicolumn{6}{|c|}{ Physical functioning } \\
\hline High & $11(9)$ & $5(39)$ & $10(19)$ & $6(10)$ & 0.001 \\
\hline Medium & $14(50)$ & $7(54)$ & $38(56)$ & $33(56)$ & \\
\hline Low & $3(11)$ & $1(8)$ & $5(9)$ & $20(34)$ & \\
\hline \multicolumn{6}{|l|}{ Co-morbidity } \\
\hline No & $8(40)$ & $3(30)$ & $10(22)$ & $5(12)$ & 0.08 \\
\hline Yes & $12(60)$ & $7(70)$ & $35(78)$ & $38(88)$ & \\
\hline \multicolumn{6}{|l|}{ Diabetes } \\
\hline No & $22(92)$ & $11(92)$ & $44(96)$ & $44(88)$ & 0.61 \\
\hline Yes & $2(8)$ & $1(8)$ & $2(4)$ & $6(12)$ & \\
\hline \multicolumn{6}{|l|}{ Osteoarthritis } \\
\hline No & $17(74)$ & $9(75)$ & $32(67)$ & $27(53)$ & 0.22 \\
\hline Yes & $6(26)$ & $3(25)$ & $16(33)$ & $24(47)$ & \\
\hline \multicolumn{6}{|l|}{ ISS stage (ISS) } \\
\hline 1 & $11(36)$ & $3(23)$ & $20(38)$ & $20(34)$ & 0.60 \\
\hline ॥ & $7(23)$ & $5(39)$ & $11(21)$ & $19(32)$ & \\
\hline III & $5(16)$ & $3(23)$ & $7(13)$ & $12(20)$ & \\
\hline unknown & $8(26)$ & $2(15)$ & $15(28)$ & $8(14)$ & \\
\hline Chemotherapy & & & & & 0.006 \\
\hline CT- & $7(23)$ & $0(0)$ & $2(4)$ & $3(5)$ & \\
\hline $\mathrm{CT}+$ & $24(77)$ & $13(100)$ & $50(96)$ & $56(95)$ & \\
\hline \multicolumn{5}{|c|}{ Number of neurotoxic } & 0.04 \\
\hline 0 & $8(26)$ & $0(0)$ & $4(8)$ & $3(5)$ & \\
\hline 1 & $10(32)$ & $7(54)$ & $27(52)$ & $29(49)$ & \\
\hline $2+$ & $13(42)$ & $6(46)$ & $21(40)$ & $27(46)$ & \\
\hline \multicolumn{6}{|c|}{ Time since last received } \\
\hline $0-3$ months & $10(42)$ & $4(31)$ & $17(35)$ & $22(42)$ & \\
\hline 4-12 months & $3(13)$ & $3(23)$ & $11(22)$ & $10(19)$ & \\
\hline$>12$ months & $11(46)$ & $6(46)$ & $21(43)$ & $21(40)$ & \\
\hline
\end{tabular}

ICPNQ: Indication for Common Toxicity Criteria grading of Peripheral Neuropathy Questionnaire. Due to rounding off percentages can exceed $100 \%$. * Ability of the ICPNQ to distinct subgroups of patients, calculated with $\mathrm{X}^{2}$ analysis. ${ }^{* *} \mathrm{P}$-value calculated with $\mathrm{X}^{2}$ analysis. 

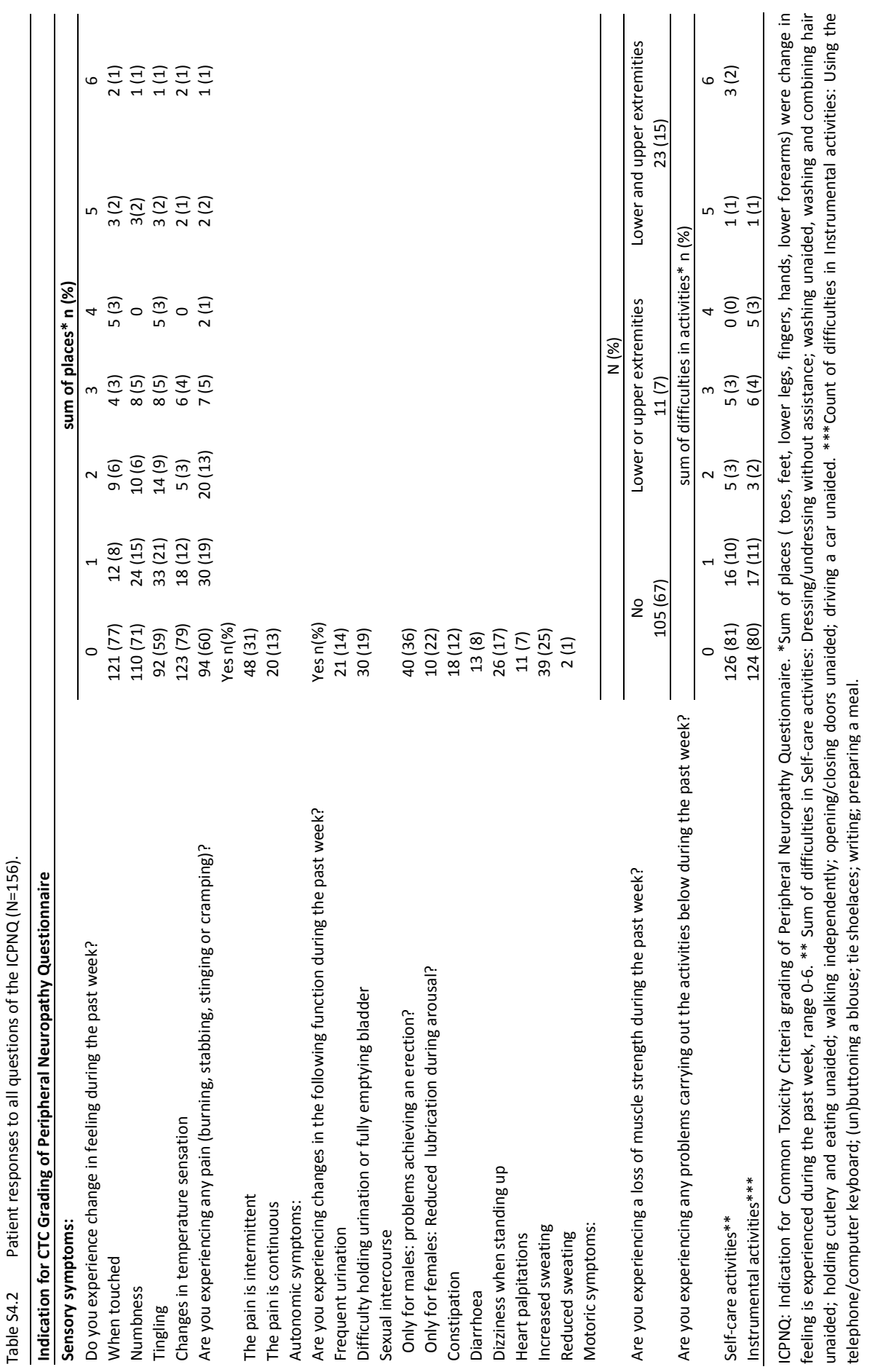


\section{PART II}

Chemotherapy-induced peripheral neuropathy and the influence of chemotherapy administration 


\section{Chapter 5}

\section{A systematic review on chronic oxaliplatin-induced peripheral neuropathy and the relation with}

oxaliplatin administration 


\section{Abstract}

\section{Purpose}

The aim of this study was to systematically review the literature on the influence of oxaliplatin administration (e.g. cumulative dose, dose intensity, number of cycles and combination regimen) on the long-term prevalence of oxaliplatin-induced peripheral neuropathy (O-IPN) at least 12 months after termination of chemotherapy.

\section{Methods}

A computerized search of literature on databases Pubmed and Cochrane was performed. Published original articles were included if they reported about long-term O-IPN and gave concomitant information about oxaliplatin therapy given to the patients. All articles were assessed for quality.

\section{Results}

We included 14 articles ( $n=3,869$ patients), and the majority of these studies were of high quality. All included patients were treated for colorectal cancer, mainly with oxaliplatin in combination with 5-fluorouracil/leucovorin. Median cumulative doses and dose intensity varied between $676 \mathrm{mg} / \mathrm{m}^{2}$ and $1,449 \mathrm{mg} / \mathrm{m}^{2}$ and 30.8 and $42.6 \mathrm{mg} / \mathrm{m}^{2} /$ week, respectively. Neuropathy assessment differed between studies, and the National Cancer Institute-Common Terminology Criteria (NCl-CTC) was used most often. The degree of neuropathy ranged from grade 0 to 3 . Only six studies directly assessed the relationship between oxaliplatin administration and neuropathy. Of these studies five did find a relation between neuropathy and higher cumulative dose, while one study did not find a relation.

\section{Conclusions}

O-IPN is still present in a great amount of patients in $\geq 12$ months after termination of therapy. A higher cumulative dose is likely to have an influence on the development of long-term O-IPN. More studies are needed that assess long-term neuropathy and oxaliplatin administration by means of validated neuropathy assessments. 


\section{Introduction}

The platinum compound oxaliplatin is a widely used cytostatic agent which has proven to be effective in various solid tumours, mainly among colorectal cancer (CRC). Oxaliplatin, first successfully used for the management of advanced CRC, is nowadays also the regimen of choice for adjuvant treatment of patients with curative resected node-positive colon cancer. ${ }^{1-5}$ Unlike cisplatin, it causes no nephrotoxicity and only mild haematological toxicity, but oxaliplatin-induced peripheral neuropathy (O-IPN) on the contrary is the most common dose-limiting side effect of oxaliplatin. ${ }^{1,2,5-8}$ The mechanism by which neuropathy is induced is unclear. Several trials have suggested that oxaliplatin accumulates in the dorsal root ganglia and produces axonal hyperexcitability and repetitive discharges due to changes in voltage-dependent $\mathrm{Na}^{+}$-channels. ${ }^{8-11}$

Oxaliplatin is known to cause two different types of neuropathy: acute and chronic neuropathy. Acute neuropathy (e.g. distal paresthesias, dysesthesias, and mild muscle contractions of hands, feet, and perioral region) is mainly cold triggered, occurs in approximately $90 \%$ of patients and reverses characteristically within a week. ${ }^{12,13}$ In addition, chronic cumulative O-IPN persists between and after treatment, and severe O-IPN resolves in approximately 13 weeks after treatment in the majority of patients. ${ }^{13}$ However, a significant proportion of patients still experience chronic neuropathy after more than a year which may have a negative influence on patients' quality of life (QOL). ${ }^{6,14-17}$ For instance, a population-based study among CRC survivors $(n=1,643)$ showed that long-term chemotherapy-induced peripheral neuropathy (CIPN) was negatively associated with QOL 2-11 years after diagnosis. ${ }^{16}$ Furthermore, a well-conducted recent study also demonstrated that O-IPN impacts emotional and physical well-being and QOL years after treatment. ${ }^{17}$ In addition, a recently conducted systematic review concerning CIPN and QOL states that CIPN may negatively influence QOL.

It is acknowledged that the degree of O-IPN is dependent on cumulative dose, duration of administration and dose intensity. ${ }^{6,8,18,19}$ Nonetheless, this knowledge is mainly based on studies concerning the development of acute neuropathy ${ }^{1,2,5}$ instead of studies reporting about chronic neuropathy more than a year after treatment. Therefore, the influence of oxaliplatin administration on the development of chronic neuropathy remains unclear. Although a recent study by Vatandoust et al. advocated that persistent grades 2 and 3 O-IPN was more common in patients who received a cumulative dose of more than $900 \mathrm{mg} / \mathrm{m}^{2}$, suggesting influence of oxaliplatin administration on long-term O-IPN. ${ }^{20}$ This interesting study correctly states that there is an increasing need to understand this long-term side effect.

Oxaliplatin is commonly regarded as the standard therapy in adjuvant and palliative chemotherapy regimens, and since the survival of CRC increases, the management of long-term side effects is becoming more important. Because there is no well-accepted 
proven therapy or neuroprotective strategy for O-IPN, it is very important to determine the influence of oxaliplatin administration on the development of this chronic neuropathy as it may have consequences in clinical decision making. Therefore, our aim is to review studies that report about the influence of oxaliplatin administration (e.g. cumulative dose, dose intensity, number of cycles and combination regimen) on the prevalence and course of O-IPN at least 12 months after cessation of oxaliplatin.

\section{Methods}

\section{Search strategy}

A computerized search of the literature through the search engines Pubmed and Cochrane was performed, using the terms 'oxaliplatin' AND 'chemotherapy' AND 'neuropathy' OR 'neurotoxicity' OR 'oxaliplatin-induced neuropathy' AND 'long term' OR 'prospective' OR 'retrospective' OR 'follow up'. References of all identified articles were checked for other relevant publications, which had not been identified through the computerized search.

\section{Selection criteria}

Studies that met the following criteria were included: (1) if O-IPN was assessed among cancer patients treated with oxaliplatin after a follow-up time of at least 12 months, (2) if information about oxaliplatin administration was available (e.g. treatment schedule, total cumulative dose and dose intensity), (3) if the publication was an original study (e.g. no review, poster abstracts, editorials, letters to the editor, etc.), (4) if they were published in peer-reviewed journals and (5) if they were written in English. Studies were excluded for the following reason: (1) if they investigated a therapeutic option or preventive strategy for O-IPN.

The described inclusion and exclusion criteria were applied to our initial 244 hits. These studies were conducted between 2003 and 2012. After careful review, 14 articles fulfilled our selection criteria and were included in this review. $3,6,7,21-31$ A flowchart of this selection procedure is shown in Figure 5.1. 


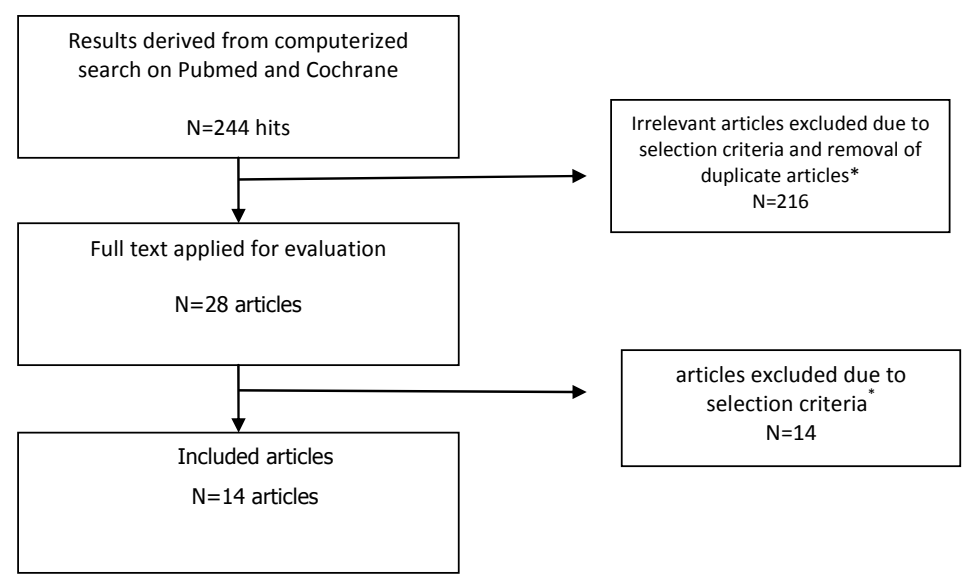

Figure 5.1 Flowchart of the selection procedure. ${ }^{*}$ indicates that the selection criteria are described in the "Methods" section.

\section{Quality assessment}

All included articles were assessed for methodological quality with a 12-item checklist of predefined criteria by two investigators (TB and FM). The checklist was based on items of recognized criteria lists for systematic reviews ${ }^{32,33}$ and on items which were considered to be relevant for the aim of our study. The checklist is presented in Table 5.1.

Each item of a selected study that matched our criteria received one point. If an item did not correspond to our criteria or was mentioned insufficiently, no points were assigned. Studies of 'high quality' were arbitrarily considered to score $75 \%$ or more of the maximum achievable score $(\geq 9)$. Studies of 'adequate quality' were considered to achieve a score between 50 and $75 \%$ (six to eight points), and studies with a score of less than six points were classified as 'low quality'. Therefore, the highest achievable score was 12 points. Disagreements between the two reviewers were solved through discussion in a consensus meeting. 
Table 5.1 List of criteria for assessing the methodological quality of studies concerning the prevalence of long-term oxaliplatin-induced peripheral neuropathy and relation to oxaliplatin administration.

Positive if with respect to

Outcomes

1. Oxaliplatin-induced neuropathy is assessed according to the $\mathrm{NCl}-\mathrm{CTC}$ criteria or by a neuropathy questionnaire

2. A neuropathy exam (e.g. neurophysiological assessment) is performed (at time of follow-up $>12$ months)

3. A description of at least two variables of oxaliplatin administration is given (e.g. oxaliplatin regime, cumulative doses, dose intensity, median number of cycles)

Study population

4. A description is included of at least two baseline variables (e.g. cancer, stage, age, sex) of participants

5. Inclusion and/or exclusion criteria are described

6. Insight in time of CIPN measurement and number of patients assessed at those time points.

7. Information is given about the degree of selection of sample (e.g. information is given about the ratio respondents versus non-respondents after at least 12 months)

Study design

8. The study size consists of at least 50 participants (arbitrarily chosen)

9. The data is prospectively gathered

10. The process of data collection is described (no points were assigned if it was only report that $\mathrm{NCl}$ CTC was recorded without mentioning by whom and how).

Results

11. The results are compared between two groups or more (e.g. different chemotherapy treatment, different cumulative doses, different time of assessment etc.)

12. Relationship between long-term neuropathy and oxaliplatin administration is described.

CIPN: chemotherapy-induced peripheral neuropathy; NCl-CTC: National Cancer Institute- Common Terminology Criteria.

\section{Results}

\section{Study characteristics}

The 14 studies included were conducted between 2003 and 2012 (Table 5.2). The included number of oxaliplatin-treated patients $(n=3,869)$ varied from 16 patients $^{6}$ to 2,710 patients. ${ }^{21}$ Patients in all studies were treated with oxaliplatin for stage II or III, $31,23-25,27,28,30,31$ or stage IV $^{6,7,25,26,29,31}$ CRC. However, an Italian study also included 15 patients with gastric cancer. ${ }^{30}$ Generally, oxaliplatin was administered in combination with 5-fluorouracil/leucovorin (5-FU/LV). ${ }^{3,67,21,23-30}$ Furthermore, combination therapy with capecitabine was given in two studies, ${ }^{25,31}$ oxaliplatin monotherapy was administered in one study ${ }^{26}$, and the exact oxaliplatin regimen was not clear in one study. ${ }^{22}$ Available median cumulative doses varied between 
$676 \mathrm{mg} / \mathrm{m}^{223,24,27}$ and $1,449 \mathrm{mg} / \mathrm{m}^{26}$, and dose intensity varied between $30.8 \mathrm{mg} / \mathrm{m}^{2} /$ week $^{23,24,27}$ and $42.6 \mathrm{mg} / \mathrm{m}^{2} /$ week. $^{30}$ Predominantly, neuropathy was assessed according to the National Cancer Institute Common Terminology Criteria (NCl-CTC), $3,6,21,24,25,27-31$ but it was also assessed with the Functional Assessment of Cancer Therapy/Gynecologic Oncology Group-Oxaliplatin Specific Neurotoxicity questionnaire (FACT/GOG-Ntx), ${ }^{23,24}$ a neuropathic symptom questionnaire, ${ }^{22}$ or neurophysiological examination (e.g. nerve conduction studies). ${ }^{6,7,22,25}$ Time of neuropathy assessment varied between 12 months $s^{6,21,28,31}$ and 8 years. ${ }^{27}$

\section{Quality assessment}

The quality scores ranged from 5 to 11 points (Table 5.2). Ten studies were of high quality, three studies were of adequate quality and one study was considered to be of low quality according the checklist.

\section{Long-term neurotoxicity and oxaliplatin administration in phase III trials}

Two phase III trials, the Multicenter International Study of Oxaliplatin/ 5-Fluorouracil/Leucovorin in the Adjuvant Treatment of Colon Cancer (MOSAIC) and the National Surgical Adjuvant Breast and Bowel Project (NSABP) C-07 trial, comparing 5-FU/LV with or without oxaliplatin (FOLFOX) in patients with CRC stage II or III, reported about the development of chronic O-IPN and oxaliplatin doses and dose intensity. The high-quality MOSAIC trial ( $n=976$ oxaliplatin-treated patients) showed neuropathy symptoms of any grade among $24.1 \%$ and grade 3 neuropathy symptoms in $0.7 \%$ of the oxaliplatin-treated patients after 18 months. ${ }^{3}$ In addition, after 48 months, grade 1, 2 and 3 neuropathy was observed in 11.9, 2.8 and $0.7 \%$, respectively. The median cumulative dose received in this trial was $810 \mathrm{mg} / \mathrm{m}^{2}$ (9.5 cycles), and dose intensity was $34.2 \mathrm{mg} / \mathrm{m}^{2} /$ week. Furthermore, the NSABP C-07 trial, in which oxaliplatin-treated patients $(n=1,200)$ received a median cumulative dose of $676 \mathrm{mg} / \mathrm{m}^{2}$ (dose intensity $30.8 \mathrm{mg} / \mathrm{m}^{2} /$ week), ${ }^{34}$ showed that 8 years after oxaliplatin treatment, overall $30.4 \%$ of the patients experienced $\geq$ grade 2 neurosensory toxicity. However, the time of O-IPN assessment was unclear, and the study was considered to be of low quality according to our checklist. ${ }^{27}$ Furthermore, a high quality sub-study of the NSABP C-07 trial ${ }^{24}$ ( $n=189$ oxaliplatin-treated patients) showed a mean clinical difference with worse scores on the FACT/GOG-Ntx in $31 \%$ of the patients $(p=0.016)$, and $13.9 \%$ of oxaliplatin-treated patients still experienced numbness and tingling in their feet after 18 months. Besides, $10 \%$ of the oxaliplatintreated patients still had unresolved neurotoxicity at 27 months of follow-up. 


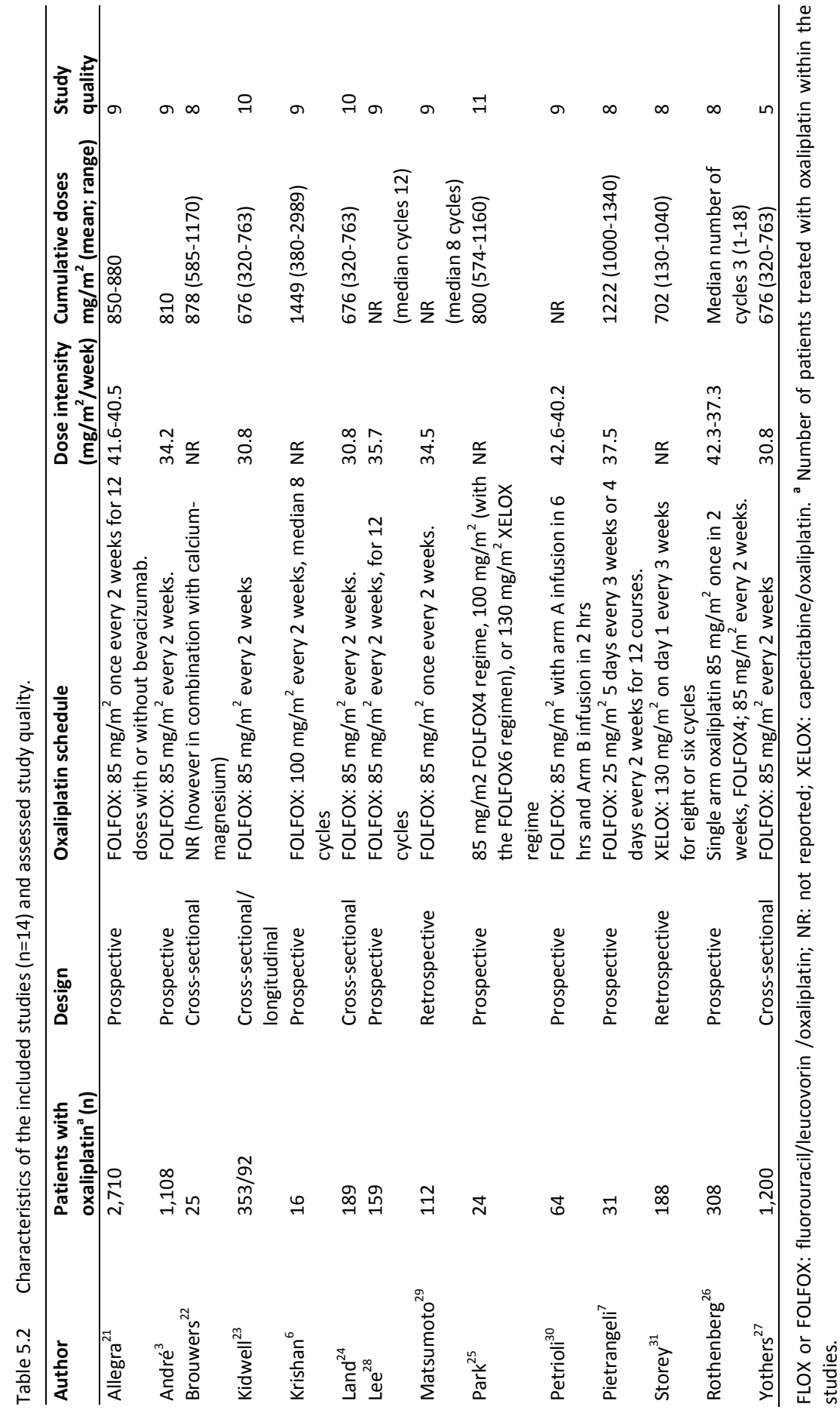


Moreover, a high-quality long-term survivorship study (LTS-01), designed by the NSABP, reported that oxaliplatin-treated patients $(n=92)$ did not have significant greater total neurotoxicity scores than 5-FU/LV-treated patients after 7 years. ${ }^{23}$ Another high-quality NSABP C-08 trial $(n=2,710)$, FOLFOX with or without bevacizumab in stage II or III CRC patients with a median cumulative dose of oxaliplatin of $850-880 \mathrm{mg} / \mathrm{m}^{2}$, demonstrated grade $\geq 2$ sensory neuropathy in $26.1 \%$ vs. $32.4 \%(p<0.01)$ in the FOLFOX and FOLFOX/bevacizumab arm, respectively, after 12 months. ${ }^{21}$ Moreover, interim 5-year data from an American trial of adequate quality showed grades 3 and 4 cumulative peripheral neuropathy in 2 and $3 \%$ of metastatic CRC patients treated with oxaliplatin monotherapy $(n=153$, dose intensity of $99.6 \%)$ or oxaliplatin in combination with 5 -FU/LV ( $n=150$, dose intensity of $87.8 \%)$. However, it is not clear when this cumulative neuropathy was assessed. ${ }^{26}$

\section{Long-term neurotoxicity and oxaliplatin administration in other studies}

Although the other eight studies were not based on phase III trials, they described long-term O-IPN in combination with information about oxaliplatin administration. Two were retrospective, five of them had a prospective design, and one had a crosssectional design. Of these studies, six ${ }^{6,7,22,25,29,31}$ did directly assess the association of oxaliplatin administration on O-IPN (Table 5.3).

The largest non-phase III studies which directly assessed the influence of oxaliplatin administration on the development of O-IPN were of retrospective design. Firstly, a retrospective high-quality Asian study showed persistent neuropathy after 12, 15 and 18 months among patients with advanced CRC ( $n=112)$. Also, grade 2 neuropathy was more often experienced in patients who had received more than 10 cycles of FOLFOX (cumulative dose $>850 \mathrm{mg} / \mathrm{m}^{2}$ ) without a break in comparison with cumulative dose of $>850 \mathrm{mg} / \mathrm{m}^{2}$ with a break between cycles. ${ }^{29}$ The other retrospective study of adequate quality $^{31}(\mathrm{n}=188)$ showed that $28 \%$ of patients had persistent neuropathy with impaired function in 7\% of patients after 12 months. Besides, neuropathy was associated with a higher median cumulative doses of $834 \mathrm{mg} / \mathrm{m}^{2}$ in symptomatic versus $702 \mathrm{mg} / \mathrm{m}^{2}$ in the asymptomatic patients ${ }^{31}$

Two prospective studies of high quality and one prospective study of adequate quality reported the association between oxaliplatin administration and long-term O-IPN. An Australian prospective study reported persistent neuropathic symptoms in $79.2 \%$ of oxaliplatin-treated patients $(n=24)$ and loss of sensory amplitude in both upper and lower limps at a median follow-up of 25 months after treatment with median cumulative oxaliplatin doses of $800 \mathrm{mg} / \mathrm{m}^{2}{ }^{25}$ Cumulative dose was a significant predictor of the development of neuropathy. ${ }^{25}$ Another Australian high-quality prospective study, with a small sample size $(n=16)$, showed that eight of the 16 patients developed chronic neuropathy after cessation of oxaliplatin and that all 
symptomatic patients received a cumulative dose of more than $1,200 \mathrm{mg} / \mathrm{m}^{2}{ }^{6}$ Only two patients developed symptomatic grades 1 and 2 neuropathy after 12 months. Additionally, a small prospective study $(n=31)$ of adequate quality demonstrated persistent neuropathy with alteration in neurophysiological tests after receiving a cumulative dose of more than $1,000 \mathrm{mg} / \mathrm{m}^{2}$. Finally, a Dutch cross-sectional study $(n=25)$ assessed neuropathy using questionnaires, neurological tests and vibration threshold measurements after a median follow-up of 18 months and reported persistent neuropathy in the majority of patients. However, no relationship between oxaliplatin dose and neuropathy could be observed. ${ }^{22}$

Table 5.3 Studies investigating the association between oxaliplatin-induced peripheral neuropathy and cumulative doses.

\begin{tabular}{|c|c|c|c|}
\hline Study & $\begin{array}{l}\text { Cumulative dose } \\
\text { in symptomatic } \\
\text { patients }\left(\mathrm{mg} / \mathrm{m}^{2}\right)\end{array}$ & Neuropathy and cumulative dose $\left(\mathrm{mg} / \mathrm{m}^{2}\right)$ & p-value \\
\hline Matsumoto $^{29}$ & $>850$ & $\begin{array}{l}\text { Grade } 2 \text { neuropathy was more often experienced in } \\
\text { patients who had received more than } 10 \text { cycles of } \\
\text { FOLFOX (cumulative dose }>850 \mathrm{mg} / \mathrm{m} 2 \text { ) without a } \\
\text { break in comparison with cumulative dose of }>850 \\
\mathrm{mg} / \mathrm{m} 2 \text { with a break between cycles }\end{array}$ & NR \\
\hline Storey $^{31}$ & $\geq 834$ & $\begin{array}{l}\text { Symptomatic patients received } 834 \mathrm{mg} / \mathrm{m}^{2} \\
\text { Asymptomatic patients received } 702 \mathrm{mg} / \mathrm{m}^{2}\end{array}$ & 0.001 \\
\hline \multirow[t]{6}{*}{ Park $^{25}$} & NR & $\begin{array}{l}\text { Cumulative dose was an important predictor of the } \\
\text { development of neuropathy, and symptom severity } \\
\text { scores were significantly correlated with cumulative } \\
\text { oxaliplatin dose at follow-up-correlation }\end{array}$ & \\
\hline & & coefficients: & 0.001 \\
\hline & & TNSc, 0.658 & 0.0005 \\
\hline & & TNSr, 0.704 & 0.002 \\
\hline & & $\mathrm{NCl}$ grade, 0.603 & 0.03 \\
\hline & & NSS, 0.453 & \\
\hline Pietrangeli $^{7}$ & $\geq 1000$ & $\begin{array}{l}\text { All } 5 \text { patients with a long follow-up and alteration in } \\
\text { neurophysiological tests receiving a cumulative dose } \\
\geq 1000 \mathrm{mg} / \mathrm{m}^{2}\end{array}$ & NR \\
\hline Krishan $^{6}$ & $1,787 \pm 219(\mathrm{mg})$ & $\begin{array}{l}\text { Symptomatic patients }(1,787 \pm 219 \mathrm{mg}) \\
\text { Asymptomatic patient group }(1,110 \pm 446 \mathrm{mg}) \text {. } \\
\text { A stronger inverse correlation was noted between } \\
\text { cumulative dose and radial SNAP amplitude } \\
\text { compared with sural SNAP amplitude (radial: } r=0.51 \text {; } \\
\text { sural: } r=0.45) \text {. }\end{array}$ & 0.03 \\
\hline Brouwers $^{22}$ & NA & $\begin{array}{l}\text { No relationship between oxaliplatin dose and } \\
\text { neuropathy could be observed }\end{array}$ & NR \\
\hline
\end{tabular}

NA: not applicable; NR: Not reported; NSS: Neuropathy Symptom Score; TNS $^{-}$: Total neuropathy scale clinical version; $\mathrm{TNS}_{\mathrm{r}}$ : Total neuropathy scale reduced version with neurophysiological measures; SNAP: Sensory nerve action potential. 
The remaining two, non-phase III, studies did not directly address the influence of oxaliplatin administration on long-term O-IPN. A study with 159 patients receiving 12 cycles with oxaliplatin (median dose intensity of $35.7 \mathrm{mg} / \mathrm{m}^{2} /$ week) reported that no patients experienced grade $\geq 3$ neuropathy, and only one patient had complaints of long-term neuropathy after 12 months. ${ }^{28}$ Another high-quality study with 64 patients, who were randomized between FOLFOX infusion in 2 or $6 \mathrm{~h}$ with dose intensity of 42.6 and 40.2, respectively, showed that only one patient still experienced grade 2 neuropathy after 12 months. ${ }^{30}$

\section{Influence of follow-up, setting and combination therapy on the development of O-IPN}

Not only oxaliplatin administration (e.g. cumulative doses and dose intensity) is considered important for the development of neuropathy. The time of neuropathy assessment, indication of therapy and type of combination therapy might also influence the degree of neuropathy. The time of neuropathy assessment varied between 12 months and 8 years in the studies. The degree of neuropathy after 12 months or longer, in studies using the $\mathrm{NCl}-\mathrm{CTC}$ for neuropathy assessment, was compared in Table 5.4. After 12 months, incidence of O-IPN grades 1 and 2 neuropathy varies between $0.6 \%{ }^{28}$ to $46 \%{ }^{29}$ of patients, and after 15 months or longer, the overall experienced neuropathy differ between $13 \%{ }^{29}$ and $79.2 \%{ }^{25}$ of patients. Furthermore, oxaliplatin has been given as adjuvant and palliative treatment. However, only one study compared O-IPN between patients with adjuvant vs. palliative treatment which are very different populations with different symptom burdens. ${ }^{31}$ They reported that adjuvant-treated patients were more affected with O-IPN (35\%) than palliative-treated patients (16\%) after 12 months. ${ }^{31}$ In addition, the majority of the adjuvant-treated patients received 7 or 8 cycles of oxaliplatin vs. 6 cycles in palliative treatment. Moreover, comparison between the other studies including stage II and III vs. stage IV CRC patients receiving adjuvant or palliative treatment, respectively, does not show differences in degree of O-IPN. However comparison is difficult as time, oxaliplatin administration and way of assessment are different. Therefore, no conclusions concerning different prevalence of O-IPN in patients receiving adjuvant vs. palliative treatment can be drawn. Further, oxaliplatin was administered in the majority of the studies in combination with 5-FU/LV, 3,6,7,21,23-30 combination therapy with capecitabine was only given in the majority of patients in one study, ${ }^{31}$ and oxaliplatin monotherapy was administered in one study of adequate quality. ${ }^{26}$ In addition, one study also included few (4\%) patients treated with capecitabine and oxaliplatin; however no comparison on neuropathy was made between combination therapies with 5 -FU/LV and capecitabine. ${ }^{25}$ The Australian study, including patients treated with oxaliplatin and capecitabine, reports that O-IPN still occurred in $28 \%$ of patients after 12 months. ${ }^{31}$ On the other hand, the largest 
study with 5-FU/LV and oxaliplatin treated patients reports grade $\geq 2$ sensory neuropathy in $26.1 \%{ }^{21}$

Table 5.4 Comparison of the degree of oxaliplatin-induced peripheral neuropathy between studies using the CTC-NCl after $\geq 12$ months of follow up

\begin{tabular}{|c|c|c|}
\hline Author & Time of O-IPN assessment & Neuropathy incidence (Grade according the $\mathrm{NCl}-\mathrm{CTC}$ ) \\
\hline \multicolumn{3}{|c|}{ Studies with O-IPN assessment after 12 months of follow-up } \\
\hline Allegra $^{21}$ & 12 months & $\begin{array}{l}\text { Grade } \geq 2 \text { sensory neuropathy in } 26.1 \% \text { vs. } 32.4 \% \text { in the } \\
\text { FOLFOX and FOLFOX/ bevacizumab arm respectively. }\end{array}$ \\
\hline Krishan $^{6}$ & 12 months & Grade 1-2: $2 / 16(12.5 \%)$ patients \\
\hline \multirow[t]{3}{*}{ Land $^{24}$} & 12 months & Grade $0-1: 95 \%$ \\
\hline & & Grade $2: 4.7 \%$ \\
\hline & & Grade 3: $0.5 \%$ \\
\hline \multirow[t]{3}{*}{$\operatorname{Lee}^{28}$} & 12 months & Grade $0: 99.4 \%$ of patients \\
\hline & & Grade $\leq 3$ : One patient $(0.6 \%)$ \\
\hline & & Grade $\geq 3: 0 \%$ of patients \\
\hline Matsumoto ${ }^{29}$ & 12 months & Grade 1-2: 51/112 (46\%) patients \\
\hline \multirow[t]{2}{*}{ Petrioli ${ }^{30}$} & 12 months & Grade $2: 1 / 64(1.6 \%)$ patients \\
\hline & & Grade 3: $0 \%$ \\
\hline \multirow[t]{5}{*}{ Storey $^{31}$} & 12 months & Overall: $28 / 101$ patients (28\%) had persistent O-IPN \\
\hline & & Grade not clear: $18 / 101(18 \%)$ patients \\
\hline & & Grade $1 ; 3 / 101(3 \%)$ patients \\
\hline & & Grade $2 ; 2 / 101(2 \%)$ patients \\
\hline & & Grade $3 ; 5 / 101(5 \%)$ patients \\
\hline \multicolumn{3}{|c|}{ Studies with O-IPN assessment after $>12$ months of follow-up } \\
\hline \multirow[t]{6}{*}{ André $^{3}$} & 18 months & Grade 1: $195 / 976$ (20\%) patients \\
\hline & & Grade 2: $34 / 976(3.5 \%)$ patients \\
\hline & & Grade $3: 7 / 976(0.7 \%)$ patients \\
\hline & 48 months & Grade 1: $11.9 \%$ \\
\hline & & Grade $2: 2.8 \%$ \\
\hline & & Grade 3: $0.7 \%$ \\
\hline \multirow[t]{3}{*}{ Matsumoto $^{29}$} & 15 months & Grade 1-2: $27 / 112$ (24\%) patients \\
\hline & 18 months & Grade $1-2: 15 / 112(13 \%)$ patients \\
\hline & 24 months & Grade $1-2: 1 / 112(0.8 \%)$ patients \\
\hline \multirow[t]{4}{*}{ Park $^{25}$} & 25 months & Any grade: $19 / 24(79.2 \%)$ patients \\
\hline & & Grade $1: 9 / 24(37.5 \%)$ patients \\
\hline & & Grade $2: 7 / 24(29.2 \%)$ patients \\
\hline & & Grade 3: $3 / 24(12.5 \%)$ patients \\
\hline Yothers $^{27}$ & 8 years & $\begin{array}{l}\text { Grade } 2+: 30.4 \% \text { of patients. } \\
\text { Time of assessment not quite clear }\end{array}$ \\
\hline
\end{tabular}

${ }^{\mathrm{a}} \mathrm{NCl}-\mathrm{CTC}$ : National Cancer Institute- Common Terminology Criteria. 


\section{Discussion}

In the 14 articles included, any degree ranging from grade 1 to 3 of O-IPN is still present in a large number of patients after at least 12 months of follow-up. However, in the majority of patients, symptoms are mild to moderate (grade 1 or 2). Although all studies reported about the oxaliplatin regimen (e.g. cumulative doses and/or dose intensity), only six studies, ${ }^{6,7,22,25,29,31}$ of which four have a rather small sample size ${ }^{6,22,24,25}$ directly assessed the influence of cumulative dose on the development of long-term O-IPN. Of these six studies, five did find an association between cumulative dose and the development of chronic O-IPN. Two retrospective studies of high and adequate quality found that neuropathy was associated with higher median cumulative doses $\left(\geq 834\right.$ and $\left.850 \mathrm{mg} / \mathrm{m}^{2}\right),{ }^{29,31}$ and three small high-quality prospective studies found similar results. ${ }^{6,14,25}$ However, another small prospective study from the Netherlands did not find a relationship between oxaliplatin dose and observed neuropathy. ${ }^{22}$ The other eight studies reported long-term O-IPN and information about oxaliplatin administration separately.

Although time of follow-up, indication of therapy and type of combination regimen might also influence the degree of neuropathy, only few studies investigated these associations. Only one adequate-quality study reported about the difference in O-IPN in patients treated in adjuvant vs. palliative setting. ${ }^{31}$ Furthermore, oxaliplatin given in combination regimen with 5-FU/LV or with capecitabine might influence the degree of O-IPN. ${ }^{5,35,36}$ The only study with oxaliplatin- and capecitabine-treated patients reports that O-IPN still occurred in $28 \%$ of patients after 12 months, ${ }^{31}$ compared to $26.1 \%$ of patients in the largest study with treatment with $5-\mathrm{FU} / \mathrm{LV} .{ }^{21}$ However comparison between studies remains difficult.

Studies resemble in the fact that they all included patients treated for stages II-IV CRC, and oxaliplatin regimen was mainly given in a combination of oxaliplatin with 5-FU/LV. However, the studies differed enormously in neuropathy assessment and presentation of results, study design, follow-up time and the included sample size. For example, the time of the neuropathy assessment differed between 12 months and 8 years after cessation of treatment with oxaliplatin and sample size varied between 16 and 2,710 patients. Furthermore, O-IPN was assessed both in patients treated in the adjuvant and palliative setting. In addition, O-IPN was mainly assessed according to the $\mathrm{NCl}-\mathrm{CTC}$; however, other questionnaires and nerve conduction examinations were also used. Different questionnaires were used, and a recent study in supportive care in cancer correctly reported that a lack of standardized questionnaires for O-IPN make it difficult to compare results between studies. ${ }^{20}$ Nonetheless, besides that the studies used different assessment tools of O-IPN, even comparison of studies using the $\mathrm{NCl}$-CTC is difficult as interobserver agreement has shown to be inadequate. ${ }^{37}$ In addition, there is no consensus on which factors (e.g. subjective or objective measurements) are most important in determining clinical severity of neuropathy. 
In spite of the fact that the methodological quality of the majority of the 14 studies was high and all these differences and lack of consensus on determining the severity of O-IPN, it remains difficult to forecast the development of O-IPN in patients.

As there is no well-accepted proven therapy for O-IPN, it is important to identify patients at risk of developing O-IPN. In our study, only two studies, ${ }^{6,22}$ reported the influence of pre-existing risk factors, e.g. pre-existing neuropathy, alcoholism and diabetes mellitus, which are supposed to be risk factors for O-IPN, and three studies excluded patients with pre-existing neuropathy or diabetes mellitus. ${ }^{25,28,30}$ As the majority of studies did not mention these risk factors, this is also a limitation of the studies included in the review. Furthermore, it is suggested that certain single nucleotide polymorphisms of voltage-gated sodium channels genes are involved in the development of oxaliplatin-induced neurotoxicity. ${ }^{10,11,38}$ As certain patients might be more at risk of developing O-IPN, it is important to identify the role of oxaliplatin administration on the development of severe O-IPN. In that way, the risk of developing severe O-IPN can be minimized by applying dose modification on time or by restraining oxaliplatin therapy in certain patients.

In summary, no firm conclusions can be drawn regarding oxaliplatin administration and the emergence of long-term O-IPN. However, a higher cumulative dose is likely to be a predicting factor for the development of long-term O-IPN. Given the heterogeneous definitions and tools utilized in the studies of O-IPN, we believe a formal framework for the definition, classification and measurements of O-IPN is necessary. Moreover, as there is no currently well-accepted proven therapy for O-IPN, it is very important to try to prevent O-IPN and to identify patients who are prone to develop severe forms of this side effect as it has a negative influence on patients' quality of life. Therefore, more and larger prospective studies are needed to assess the development of long-term O-IPN in relation to these risk factors and oxaliplatin administration. Consequently in the future, cancer treatment might become more personalized as clinicians might decide to restrain oxaliplatin treatment for certain patients who are at risk of developing long-term severe neuropathy. 


\section{References}

1. de Gramont A, Figer A, Seymour $M$, Homerin $M$, Hmissi A, Cassidy J, Boni C, Cortes-Funes $H$, Cervantes A, Freyer G, Papamichael D, Le Bail N, Louvet C, Hendler D, de Braud F, Wilson C, Morvan F, Bonetti A. Leucovorin and fluorouracil with or without oxaliplatin as first-line treatment in advanced colorectal cancer. J Clin Oncol 2000;18:2938-2947

2. Andre T, Boni C, Mounedji-Boudiaf L, Navarro M, Tabernero J, Hickish T, Topham C, Zaninelli M, Clingan P, Bridgewater J, Tabah-Fisch I, de Gramont A, Multicenter International Study of Oxaliplatin/5-Fluorouracil/Leucovorin in the Adjuvant Treatment of Colon Cancer I. Oxaliplatin, fluorouracil, and leucovorin as adjuvant treatment for colon cancer. N Engl J Med 2004;350: 2343-2351.

3. Andre T, Boni C, Navarro M, Tabernero J, Hickish T, Topham C, Bonetti A, Clingan P, Bridgewater J, Rivera F, de Gramont A. Improved overall survival with oxaliplatin, fluorouracil, and leucovorin as adjuvant treatment in stage II or III colon cancer in the MOSAIC trial. J Clin Oncol 2009;27:3109-3116.

4. Haller DG, Tabernero J, Maroun J, de Braud F, Price T, Van Cutsem E, Hill M, Gilberg F, Rittweger K, Schmoll HJ. Capecitabine plus oxaliplatin compared with fluorouracil and folinic acid as adjuvant therapy for stage III colon cancer. J Clin Oncol 2011;29:1465-1471.

5. Ducreux M, Bennouna J, Hebbar M, Ychou M, Lledo G, Conroy T, Adenis A, Faroux R, Rebischung C, Bergougnoux L, Kockler L, Douillard JY, Centers GIGotFA-C. Capecitabine plus oxaliplatin (XELOX) versus 5-fluorouracil/leucovorin plus oxaliplatin (FOLFOX-6) as first-line treatment for metastatic colorectal cancer. Int J Cancer 2011;128:682-690.

6. Krishnan AV, Goldstein D, Friedlander M, Kiernan MC. Oxaliplatin-induced neurotoxicity and the development of neuropathy. Muscle Nerve 2005;32:51-60.

7. Pietrangeli A, Leandri M, Terzoli E, Jandolo B, Garufi C. Persistence of high-dose oxaliplatin-induced neuropathy at long-term follow-up. Eur Neurol 2006;56:13-16.

8. Pasetto LM, D'Andrea MR, Rossi E, Monfardini S. Oxaliplatin-related neurotoxicity: how and why? Criti Rev Oncol Hematol 2006;59:159-168.

9. Park SB, Lin CS, Krishnan AV, Goldstein D, Friedlander ML, Kiernan MC. Dose effects of oxaliplatin on persistent and transient $\mathrm{Na}+$ conductances and the development of neurotoxicity. PloS One 2011;6:e18469.

10. Faber CG, Hoeijmakers JG, Ahn HS, Cheng X, Han C, Choi JS, Estacion M, Lauria G, Vanhoutte EK, Gerrits MM, Dib-Hajj S, Drenth JP, Waxman SG, Merkies IS. Gain of function Nanu1.7 mutations in idiopathic small fiber neuropathy. Ann Neurol 2012;71:26-39.

11. Faber CG, Lauria G, Merkies IS, Cheng X, Han C, Ahn HS, Persson AK, Hoeijmakers JG, Gerrits MM, Pierro T, Lombardi R, Kapetis D, Dib-Hajj SD, Waxman SG. Gain-of-function Nav1.8 mutations in painful neuropathy. Proc Natl Acad Sci U S A 2012;109:19444-19449.

12. Beijers AJ, Jongen JL, Vreugdenhil G. Chemotherapy-induced neurotoxicity: the value of neuroprotective strategies. Neth J Med 2012;70:18-25.

13. Grothey A (2003) Oxaliplatin-safety profile: neurotoxicity. Semin Oncol 2003;30(4 Suppl 15):5-13.

14. Ocean A, Vahdat L. Chemotherapy-induced peripheral neuropathy: pathogenesis and emerging therapies. Support Care Cancer 2004;12:619-625.

15. Quasthoff S, Hartung H. Chemotherapy-induced peripheral neuropathy. J Neurol 2002;249:9-17.

16. Mols F, Beijers T, Lemmens V, van den Hurk CJ, Vreugdenhil G, van de Poll-Franse LV. Chemotherapyinduced neuropathy and its association with quality of life among 2- to 11-year colorectal cancer survivors: Results from the population-based PROFILES registry. J Clin Oncol 2013;31:2699-707.

17. Tofthagen C, Donovan KA, Morgan MA, Shibata D, Yeh Y. Oxaliplatin-induced peripheral neuropathy's effects on health-related quality of life of colorectal cancer survivors. Support Care Cancer 2013;21:3307-3313

18. Cavaletti G, Tredici G, Petruccioli MG, Donde E, Tredici P, Marmiroli P, Minoia C, Ronchi A, Bayssas M, Etienne GG. Effects of different schedules of oxaliplatin treatment on the peripheral nervous system of the rat. Eur J Cancer 2001; 37 (18):2457-2463 
19. Velasco R, Bruna J, Briani C, Argyriou AA, Cavaletti G, Alberti P, Frigeni B, Cacciavillani M, Lonardi S, Cortinovis D, Cazzaniga M, Santos C, Kalofonos HP. Early predictors of oxaliplatin-induced cumulative neuropathy in colorectal cancer patients. J Neurol Neurosurg Psyciatry 2013;85(4):392-398.

20. Vatandoust S, Joshi R, Pittman KB, Esterman A, Broadbridge V, Adams J, Singhal N, Yeend S, Price TJ. A descriptive study of persistent oxaliplatin-induced peripheral neuropathy in patients with colorectal cancer. Support Care Cancer 2013;22(2):513-518.

21. Allegra CJ, Yothers G, O'Connell MJ, Sharif S, Colangelo LH, Lopa SH, Petrelli NJ, Goldberg RM, Atkins JN, Seay TE, Fehrenbacher L, O'Reilly S, Chu L, Azar CA, Wolmark N (2009) Initial safety report of NSABP C-08: A randomized phase III study of modified FOLFOX6 with or without bevacizumab for the adjuvant treatment of patients with stage II or III colon cancer. J Clin Oncol 2009;27(20):3385-3390.

22. Brouwers EE, Huitema AD, Boogerd W, Beijnen JH, Schellens JH. Persistent neuropathy after treatment with cisplatin and oxaliplatin. Acta Oncol 2009; 48 (6):832-841.

23. Kidwell KM, Yothers G, Ganz PA, Land SR, Ko CY, Cecchini RS, Kopec JA, Wolmark N (2012) Long-term neurotoxicity effects of oxaliplatin added to fluorouracil and leucovorin as adjuvant therapy for colon cancer: results from National Surgical Adjuvant Breast and Bowel Project trials C-07 and LTS-01. Cancer 118 (22):5614-5622.

24. Land SR, Kopec JA, Cecchini RS, Ganz PA, Wieand HS, Colangelo LH, Murphy K, Kuebler JP, Seay TE, Needles BM, Bearden JD, 3rd, Colman LK, Lanier KS, Pajon ER, Jr., Cella D, Smith RE, O'Connell MJ, Costantino JP, Wolmark N. Neurotoxicity from oxaliplatin combined with weekly bolus fluorouracil and leucovorin as surgical adjuvant chemotherapy for stage II and III colon cancer: NSABP C-07. J Clin Oncol 2007; 25 (16):2205-2211.

25. Park SB, Lin CS, Krishnan AV, Goldstein D, Friedlander ML, Kiernan MC. Long-term neuropathy after oxaliplatin treatment: challenging the dictum of reversibility. Oncologist 2011;16 (5):708-716.

26. Rothenberg ML, Oza AM, Bigelow RH, Berlin JD, Marshall JL, Ramanathan RK, Hart LL, Gupta S, Garay CA, Burger BG, Le Bail N, Haller DG. Superiority of oxaliplatin and fluorouracil-leucovorin compared with either therapy alone in patients with progressive colorectal cancer after irinotecan and fluorouracil-leucovorin: interim results of a phase III trial. J Clin Oncol 2003;21 (11):2059-2069.

27. Yothers G, O'Connell MJ, Allegra CJ, Kuebler JP, Colangelo LH, Petrelli NJ, Wolmark N. Oxaliplatin as adjuvant therapy for colon cancer: updated results of NSABP C-07 trial, including survival and subset analyses. . J Clin Oncol 2011; 29 (28):3768-3774.

28. Lee P, Park YS, Ji JF, Fu YT, Ratanatharathorn V. Safety and tolerability of FOLFOX4 in the adjuvant treatment of colon cancer in Asian patients: The MASCOT study. Asia-Pac J Clin Oncol 2009; 5: 101-110

29. Matsumoto S, Nishimura T, Kanai M, Mori Y, Nagayama S, Kawamura J, Nomura A, Miyamoto S, Kitano T, Ishiguro H, Yanagihara K, Teramukai S, Sakai Y, Chiba T, Fukushima M. Safety and efficacy of modified FOLFOX6 for treatment of metastatic or locally advanced colorectal cancer. A singleinstitution outcome study. Chemotherapy 2008; 54 (5):395-403.

30. Petrioli R, Pascucci A, Francini E, Marsili S, Sciandivasci A, Tassi R, Civitelli S, Tanzini G, Lorenzi M, Francini G. Neurotoxicity of FOLFOX-4 as adjuvant treatment for patients with colon and gastric cancer: a randomized study of two different schedules of oxaliplatin. Cancer Chemother Pharmacol 2008; 61 (1):105-111.

31. Storey DJ, Sakala M, McLean CM, Phillips HA, Dawson LK, Wall LR, Fallon MT, Clive S. Capecitabine combined with oxaliplatin (CapOx) in clinical practice: how significant is peripheral neuropathy? Ann Oncol 2010; 21 (8):1657-1661.

32. Mols F, Vingerhoets AJ, Coebergh JW, van de Poll-Franse LV. Quality of life among long-term breast cancer survivors: a systematic review. Eur J Cancer 2005; 41 (17):2613-2619.

33. Vandenbroucke JP, von Elm E, Altman DG, Gotzsche PC, Mulrow CD, Pocock SJ, Poole C, Schlesselman JJ, Egger M, Initiative S. Strengthening the Reporting of Observational Studies in Epidemiology (STROBE): explanation and elaboration. Epidemiology 2007; 18 (6):805-835. 
34. Kuebler JP, Wieand HS, O'Connell MJ, Smith RE, Colangelo LH, Yothers G, Petrelli NJ, Findlay MP, Seay TE, Atkins JN, Zapas JL, Goodwin JW, Fehrenbacher L, Ramanathan RK, Conley BA, Flynn PJ, Soori G, Colman LK, Levine EA, Lanier KS, Wolmark N. Oxaliplatin combined with weekly bolus fluorouracil and leucovorin as surgical adjuvant chemotherapy for stage II and III colon cancer: results from NSABP C07. J Clin Oncol 2007; 25 (16):2198-2204.

35. Hoff PM, Saad ED, Costa F, Coutinho AK, Caponero R, Prolla G, Gansl RC. Literature review and practical aspects on the management of oxaliplatin-associated toxicity. Clin Colorectal Cancer 2012; $11(2): 93-100$.

36. Argyriou AA, Velasco R, Briani C, Cavaletti G, Bruna J, Alberti P, Cacciavillani M, Lonardi S, Santos C, Cortinovis D, Cazzaniga M, Kalofonos HP. Peripheral neurotoxicity of oxaliplatin in combination with 5-fluorouracil (FOLFOX) or capecitabine (XELOX): a prospective evaluation of 150 colorectal cancer patients. Ann Oncol 2012; 23 (12):3116-3122.

37. Postma TJ, Heimans JJ, Muller MJ, Ossenkoppele GJ, Vermorken JB, Aaronson NK. Pitfalls in grading severity of chemotherapy-induced peripheral neuropathy. Ann Oncol 1998; 9 (7):739-744

38. Argyriou AA, Cavaletti G, Antonacopoulou A, Genazzani AA, Briani C, Bruna J, Terrazzino S, Velasco R, Alberti P, Campagnolo M, Lonardi S, Cortinovis D, Cazzaniga M, Santos C, Psaromyalou A, Angelopoulou A, Kalofonos HP. Voltage-gated sodium channel polymorphisms play a pivotal role in the development of oxaliplatin-induced peripheral neurotoxicity: Results from a prospective multicenter study. Cancer; 2013: 119(19):3570-3577. 


\section{Chapter 6}

Peripheral neuropathy in colorectal cancer survivors:

The influence of oxaliplatin administration. Results from the population-based PROFILES registry

Beijers AJ, Mols F, Tjan-Heijnen VC, Faber CG, van de Poll-Franse LV, Vreugdenhil G Acta Oncol 2015;54:463-469 


\section{Abstract}

\section{Background}

Chemotherapy-induced peripheral neuropathy (CIPN) is a dose-limiting side effect of oxaliplatin which can negatively influence quality of life. We aimed to study the influence of cumulative dose, dose schedule and dose reductions of adjuvant oxaliplatin on long-term severity and prevalence of CIPN among colorectal cancer (CRC) survivors.

\section{Materials and methods}

In total 207 patients, diagnosed with CRC between 2000 and 2009 who underwent adjuvant treatment with oxaliplatin, were included. They completed the EORTC QLQ-CIPN20 2-11 years after diagnosis. Data on oxaliplatin administration and acute neuropathy during treatment were extracted from the medical files. Subscales were analyzed with analysis of covariance and neuropathy symptoms with logistic regression analysis.

\section{Results}

Patients who received cumulative oxaliplatin dose of $\geq 842 \mathrm{mg} / \mathrm{m}^{2}$ had a significantly worse EORTC QLQ-CIPN20 sensory score compared to those who received a low cumulative dose of $<421 \mathrm{mg} / \mathrm{m}^{2}$ (mean 19 vs. $8 ; \mathrm{p}=0.02$ ). They more often reported tingling toes/feet ( $13 \% \mathrm{vs.} 2 \%$ respectively; $p=0.01$ ). Dose intensity and time delay did not influence the occurrence of CIPN. Patients receiving a dose reduction because of neuropathy $(n=50)$ reported a significantly worse sensory score at very similar cumulative doses, than those who did not receive a dose reduction because of neuropathy ( $n=96)$ (mean 21 vs. $15 ; p=0.01$ ).

\section{Conclusion}

Cumulative dose of oxaliplatin is associated with long-term CIPN. The risk of developing longterm CIPN could only be reduced by decreasing the cumulative dose, whereas delay probably is not beneficial. Patients receiving a dose reduction because of acute neuropathy, are still at risk of developing long-term CIPN. Future studies should focus on identifying patients who are at risk of developing CIPN. 


\section{Introduction}

Treatment of colorectal cancer (CRC) has considerably changed over the past decades. Oxaliplatin first successfully used in the management of advanced CRC, is nowadays the regimen of choice for adjuvant treatment of patients with curatively resected node-positive CRC with improved survival rates as a consequence. ${ }^{1}$ Therefore the use of oxaliplatin has been increased and more patients are living with its long-term side effects. Especially chemotherapy-induced peripheral neuropathy (CIPN), as the most common dose-limiting side effect of oxaliplatin, is of major concern and can have a negative influence on patients' quality of life., ${ }^{2,3}$

Axonal hyperexcitability and repetitive discharges due to changes in voltage-gated sodium and/or potassium channels and accumulation of oxaliplatin in the dorsal root ganglia with neuronal damage seem to cause the symptoms of acute and chronic oxaliplatin-induced neuropathy, respectively. ${ }^{4-7}$ The symptoms of acute neuropathy, which occur in approximately $90 \%$ of patients, reverse characteristically within a week. ${ }^{1}$ Chronic neuropathy, however, persists in $60 \%$ of patients a year or longer after the cessation of chemotherapy. ${ }^{2,8,9}$

The severity of CIPN is dependent on cumulative dose, duration of administration and dose intensity. ${ }^{9-11}$ Previously, we reported that neuropathy symptoms were still seen two to eleven years after oxaliplatin treatment in CRC patients. ${ }^{2}$ However, it is difficult to identify patients at risk for severe CIPN and information about cumulative doses, dose intensity and dose schedule was not available in our previous study. Therefore, we next aimed to study the influence of cumulative dose, dose schedule and dose reductions on long-term severity and prevalence of CIPN among CRC survivors.

\section{Materials and methods}

\section{Setting and participants}

Our prospective yearly survey was set up in December 2010 with the goal to evaluate various patient-reported outcomes, including neuropathy, among CRC survivors. All individuals diagnosed with CRC between 2000 and 2009 as registered in the Eindhoven Cancer Registry (ECR) in the Netherlands received the second yearly questionnaire in December 2011. The data presented in this paper are thus based upon the second data collection wave which included a CIPN questionnaire. A detailed description of the data collection was published earlier. ${ }^{2}$ Data collection was performed within PROFILES. ${ }^{12}$ Of the respondents, 506 (31\%) patients were treated with chemotherapy, including 207 (41\%) patients who underwent adjuvant treatment with oxaliplatin. After permission from the attending oncologists, we additionally collected information on the actually delivered oxaliplatin treatment for those 
adjuvantly treated according to the ECR $(\mathrm{N}=207)$, and these data are presented in this paper. This study was approved by a certified Medical Ethics Committee. All patients gave written informed consent.

\section{Data collection}

\section{Socio-demographic and clinical characteristics}

Survivors' socio-demographic and clinical information were available from the ECR which routinely collects data like date of diagnosis, tumor stage and treatment. Comorbidity at time of the study was assessed with the adapted Self-administered Comorbidity Questionnaire.

\section{Treatment with oxaliplatin}

Complementary information about the oxaliplatin administration, such as combination regimen, number of received chemotherapy cycles, dose of oxaliplatin per cycle, and timing and reasons for dose reduction were collected from patients' medical files.

\section{Chemotherapy-induced neuropathy}

Long-term CIPN was assessed with the European Organization for Research and Treatment of Cancer (EORTC) Quality of Life Questionnaire (QLQ)-CIPN20 $0^{13}$ which contains three subscales assessing sensory, motor, and autonomic symptoms. Each item is measured on a Likert scale ranging from 'not at all' (1) to 'very much' (4). Scores were transformed to a 0-100 scale with higher scores representing more complaints.

In addition, data about acute neuropathy complaints shortly after treatment with oxaliplatin were collected from the patients' medical files. It was defined as having any kind of sensory, motor or autonomic neuropathy as documented by the oncologist during outpatient clinical contact and/or reported according to the National Cancer Institute (NCl)-Common Toxicity Criteria (CTC) after treatment with oxaliplatin.

\section{Statistical analyses}

Patients were divided into categories of received cumulative dose; low $<421 \mathrm{mg} / \mathrm{m}^{2}$; moderate $421-842 \mathrm{mg} / \mathrm{m}^{2}$; high $\geq 842 \mathrm{mg} / \mathrm{m}^{2}, 14-16$ which correspond approximately to 1-3, 3-6 and 7 or more cycles of oxaliplatin in combination with capecitabine (CAPOX) and 1-5, 5-10 or $\geq 10$ cycles of oxaliplatin in combination with 5-fluorouracil/leucovorin (5-FU/LV) (FOLFOX). Clinical characteristics were compared between categories of cumulative dose using ANOVA for continuous data and $X^{2}$ analyses for categorical 
variables. Neuropathy symptoms that bothered patients the most were reported according the EORTC QLQ-CIPN20 answer categories 'quite a bit' or 'very much' combined. Logistic regression analyses were performed to detect differences regarding experienced neuropathy problems in the past week between the different categories of cumulative doses, treatment regimens and between patients with and without documented acute neuropathy and applied dose reduction. Mean total scores on the EORTC QLQ-CIPN20 were compared between categories of cumulative dose with analysis of covariance (ANCOVA). In addition, subgroup analysis of the EORTC QLQ-CIPN20 subscales and separate questions in patients treated with CAPOX or FOLFOX were done. Confounding background variables that might interfere with general nervous system or physical functioning were determined a priori and chosen to be diabetes mellitus (DM), osteoarthritis and rheumatoid arthritis. Furthermore, analyses were adjusted for time since last course with chemotherapy treatment as this influences the evolution of CIPN. Logistic regression analyses between regimens of chemotherapy and those for applied dose reduction were also adjusted for received cumulative dose, but not for rheumatoid arthritis. All test were two-sided, considered significant if $p<0.05$, and were performed using SPSS (version 19.0).

\section{Results}

\section{Socio-demographic and clinical characteristics}

In total, 1643 CRC patients were included in the initial study with a response rate of $83 \%$. There were no differences in socio-demographic and clinical characteristics between respondents, non-respondents and those with unverified addresses. ${ }^{2}$ Patients who were treated with chemotherapy $(n=506)$ were significantly younger, diagnosed more recently, and more often with colon cancer compared with those treated without chemotherapy. ${ }^{2}$ Patients, who underwent treatment with oxaliplatin $(n=207)$, were in $83 \%$ diagnosed with colon cancer, with a mean 4.0 years (SD 1.2) follow-up since diagnosis and $84 \%$ had stage III disease. Patients who received a higher cumulative dose of oxaliplatin were more often male ( $66 \%$ vs. $36 \%, p=0.01$ ) and were significantly younger than patients who received a lower cumulative dose (63 vs. 69 years, $p=0.01$ ) (Table 6.1).

\section{Treatment with oxaliplatin}

Oxaliplatin was mainly given in combination with capecitabine (CAPOX) ( $n=173,84 \%$ ) in a mean cumulative dose of $695 \mathrm{mg} / \mathrm{m}^{2}$ (SD 273) (Table 6.2). Any dose reduction had taken place in 144 patients (70\%) and was applied because of neurotoxicity in $38 \%$ of patients. 
Table 6.1 Sociodemographic and clinical characteristics of colorectal cancer survivors ${ }^{\mathrm{a}}$ stratified by categories of cumulative dose of oxaliplatin ${ }^{\mathrm{b}}$.

\begin{tabular}{|c|c|c|c|c|}
\hline & Categories & f received cumulat & e dose of oxalipl & $\operatorname{tin}^{b}$ \\
\hline & $\begin{array}{c}\text { Low } \\
\left(<421 \mathrm{mg} / \mathrm{m}^{2}\right) \\
n=36\end{array}$ & $\begin{array}{c}\text { Moderate } \\
\left(421-842 \mathrm{mg} / \mathrm{m}^{2}\right) \\
n=96\end{array}$ & $\begin{array}{c}\text { High } \\
\left(\geq 842 \mathrm{mg} / \mathrm{m}^{2}\right) \\
n=68\end{array}$ & p-value \\
\hline Mean age at time of survey ( \pm SD) & $68.9(7.9)$ & $65.4(9.4)$ & $63.0(9.4)$ & 0.01 \\
\hline Age at time of survey (\%) & & & & 0.02 \\
\hline$<60$ years & $4(11)$ & 18 (19) & $21(31)$ & \\
\hline $60-69$ years & $15(42)$ & $47(49)$ & $34(50)$ & \\
\hline$>70$ years & $17(47)$ & $318(32)$ & $13(19)$ & \\
\hline Mean years since diagnosis $( \pm S D)$ & $4.1(1.4)$ & $3.9(1.2)$ & $4.2(1.3)$ & 0.27 \\
\hline Years since diagnosis (\%) & & & & 0.60 \\
\hline $1-5$ years & $27(75)$ & $79(82)$ & $53(78)$ & \\
\hline $5-10$ years & $9(25)$ & $17(18)$ & $15(22)$ & \\
\hline Gender (\%) & & & & 0.01 \\
\hline Male & $13(36)$ & $57(59)$ & $45(66)$ & \\
\hline Female & $23(64)$ & $39(41)$ & $23(34)$ & \\
\hline Location of tumor (\%) & & & & 0.78 \\
\hline Colon & $31(86)$ & $78(82)$ & $57(84)$ & \\
\hline Rectal & $5(14)$ & 18 (19) & $11(16)$ & \\
\hline TNM stage (\%) & & & & 0.73 \\
\hline $\mathrm{I}$ & $0(0)$ & $3(3)$ & $0(0)$ & \\
\hline II & $3(8)$ & $9(9)$ & $7(10)$ & \\
\hline III & $31(86)$ & $81(84)$ & $59(87)$ & \\
\hline IV & $1(3)$ & $2(2)$ & $1(1)$ & \\
\hline Unknown & $1(3)$ & $0(0)$ & $1(2)$ & \\
\hline Number of comorbid conditions (\%) & & & & 0.63 \\
\hline None & $9(25)$ & $21(22)$ & $21(31)$ & \\
\hline 1 & $11(31)$ & $35(37)$ & $18(27)$ & \\
\hline $2+$ & $12(33)$ & $37(39)$ & $27(40)$ & \\
\hline Unknown & $4(1)$ & $3(3)$ & $2(3)$ & \\
\hline Most frequent conditions (\%) & & & & \\
\hline High blood pressure & $12(33)$ & $36(36)$ & $22(32)$ & 0.78 \\
\hline Osteoarthritis & $9(25)$ & $20(21)$ & $12(18)$ & 0.53 \\
\hline Back pain & $6(17)$ & $22(24)$ & $13(19)$ & 0.77 \\
\hline Heart disease & $7(19)$ & $12(13)$ & $7(10)$ & 0.30 \\
\hline Diabetes mellitus & $4(11)$ & $12(13)$ & $15(22)$ & 0.21 \\
\hline $\mathrm{BMI}\left(\mathrm{kg} / \mathrm{m}^{2}\right)(\%)$ & & & & 0.61 \\
\hline$<18.4$ (underweight) & $0(0)$ & $1(1)$ & $0(0)$ & \\
\hline 18.5 to 22.9 (normal) & $6(17)$ & $11(11)$ & $4(6)$ & \\
\hline 23.0 to 27.5 (normal) & $16(44)$ & $46(48)$ & 40 (59) & \\
\hline 27.5 to 30 (overweight) & $6(17)$ & $19(20)$ & $12(18)$ & \\
\hline$>30$ (obese) & $8(22)$ & $19(20)$ & $12(18)$ & \\
\hline Current occupation status (\%) & & & & 0.13 \\
\hline Employed & $4(11)$ & $20(21)$ & $19(28)$ & \\
\hline Not employed/retired & $307(83)$ & $76(79)$ & $47(68)$ & \\
\hline
\end{tabular}

Some variables exceed $100 \%$ due to rounding off. ${ }^{a} n=200:$ Cumulative dose was missing in 7 patients who had been treated with adjuvant oxaliplatin. ${ }^{b}$ Categories of received cumulative dose of oxaliplatin; Low $<421 \mathrm{mg} / \mathrm{m}^{2}$, Moderate $421 \mathrm{mg} / \mathrm{m}^{2}-842 \mathrm{mg} / \mathrm{m}^{2}$, High $\geq 842 \mathrm{mg} / \mathrm{m}^{2}$. 
Table 6.2 Clinical characteristics of oxaliplatin administration, $n=207$.

\begin{tabular}{|c|c|}
\hline & $n=\mathbf{2 0 7}$ \\
\hline \multicolumn{2}{|l|}{ Treatment regimen $(n, \%)$} \\
\hline CAPOX & $173(84)$ \\
\hline FOLFOX & $27(13)$ \\
\hline Capecitabine/oxaliplatin/bevacizumab & $7(3)$ \\
\hline \multicolumn{2}{|l|}{ Total received cycles (median, range) } \\
\hline Median & 6 \\
\hline Range & $1-12$ \\
\hline \multicolumn{2}{|l|}{ Duration of treatment with oxaliplatin, weeks ${ }^{a}$} \\
\hline Mean & 15 \\
\hline Range & $0-32$ \\
\hline \multicolumn{2}{|l|}{ Cumulative dose, $\mathrm{mg} / \mathrm{m}^{2}$} \\
\hline Mean & 695 \\
\hline SD & 273 \\
\hline \multicolumn{2}{|l|}{ Total cumulative dose in categories ( $n, \%)$} \\
\hline $0-421 \mathrm{mg} / \mathrm{m}^{2}$ & $36(17)$ \\
\hline $421-842 \mathrm{mg} / \mathrm{m}^{2}$ & $96(46)$ \\
\hline$>842 \mathrm{mg} / \mathrm{m}^{2}$ & $68(33)$ \\
\hline unknown & $7(3)$ \\
\hline \multicolumn{2}{|l|}{ Dose intensity, $\mathrm{mg} / \mathrm{m}^{2} /$ week (median) } \\
\hline CAPOX & 41.6 \\
\hline FOLFOX & 37.0 \\
\hline \multicolumn{2}{|l|}{ Dose reduction applied ( $n, \%)$} \\
\hline Yes & $144(70)$ \\
\hline No & $56(27)$ \\
\hline Unknown & $7(3)$ \\
\hline \multicolumn{2}{|l|}{ Course number of first dose reduction } \\
\hline Median & 4 \\
\hline SD & 2.2 \\
\hline \multicolumn{2}{|l|}{ Type of dose reduction ( $n, \%)$} \\
\hline Discontinuation of oxaliplatin & $51(35)$ \\
\hline Dose rate reduction & $20(10)$ \\
\hline Discontinuation after dose rate reduction & $23(11)$ \\
\hline Interval prolongation & $20(10)$ \\
\hline \multicolumn{2}{|l|}{ Dose reduction because of neurotoxicity ( $n, \%)$} \\
\hline Yes & $54(38)$ \\
\hline No & $90(62)$ \\
\hline
\end{tabular}

CAPOX: oxaliplatin $\left(130 \mathrm{mg} / \mathrm{m}^{2}\right)$ every 3 weeks in combination with capecitabine $\left(1000 \mathrm{mg} / \mathrm{m}^{2}\right)$ twice every day during 2 weeks ; FOLFOX: oxaliplatin $\left(85 \mathrm{mg} / \mathrm{m}^{2}\right)$ in combination with 5-FU/LV every 2 weeks. ${ }^{\mathrm{a}}$ Duration of administration: weeks between date first gift of oxaliplatin and date of last gift of oxaliplatin.

\section{Chemotherapy-induced neuropathy}

The neuropathy subscale-related symptoms that bothered patients the most during the past week were mainly sensory and consisted of tingling toes or feet $(30 \%)$, numbness in toes or feet $(19 \%)$, tingling hands or fingers $(15 \%)$, burning or shooting pain in the toes or feet $(13 \%)$, trouble hearing $(13 \%)$ and the autonomic symptom trouble getting or maintaining an erection ( $36 \%$ of male patients). 
Analysis among categories of cumulative doses of oxaliplatin showed that patients who received a cumulative oxaliplatin dose of $\geq 842 \mathrm{mg} / \mathrm{m}^{2}$ had significantly worse EORTC QLQ-CIPN20 sensory scores than those who received a low cumulative dose of $<421 \mathrm{mg} / \mathrm{m}^{2}$ (mean 19 vs. 8; $\mathrm{p}=0.02$ ) (Figure 6.1). No differences in the other EORTC QLQ-CIPN20 subscales were observed.

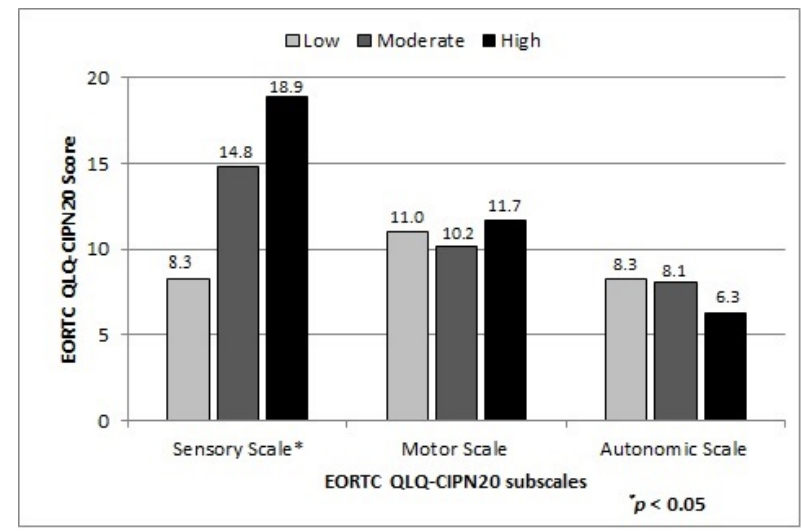

Figure 6.1 Comparison of EORTC QLQ-CIPN20 subscales according to categories of received cumulative dose of oxaliplatin ${ }^{\text {}}$. Difference of mean EORTC QLQ-CIPN subscale scores between categories of cumulative dose, analyzed with analysis of covariance adjusted for diabetes mellitus, osteoarthritis, rheumatoid arthritis and time since last course of chemotherapy. ${ }^{\text {a }}$ Categories of cumulative dose: Low $<421 \mathrm{mg} / \mathrm{m}^{2}$, Moderate $421 \mathrm{mg} / \mathrm{m}^{2}-842 \mathrm{mg} / \mathrm{m}^{2}$, High $\geq 842 \mathrm{mg} / \mathrm{m}^{2}$. $* p$-value<0.05: Significant difference between high and low cumulative dose of oxaliplatin. Standard deviations according to categories of cumulative dose; Sensory Scale: 7.1, 14.1, 19.3 respectively; Motor Scale: 13.8, 11.3, 16.9 respectively; Autonomic Scale: 14.3, 13.6, 10.9 respectively.

Logistic regression analysis of the individual EORTC QLQ-CIPN20 questions showed that tingling toes or feet were more often reported by patients who received a higher cumulative dose in comparison with low cumulative dose respectively (13 vs. $2 \%$; $\mathrm{p}=0.01$ ) (Supplementary Table $\mathrm{S6.1}$, to be found online at http://informahealthcare.com/doi/abs/10.3109/0284186X.2014.980912). In addition, trouble with standing or walking was significantly more often observed in patients treated with a high vs. moderate cumulative dose ( 6 vs. $3 \% ; p=0.03$ ).

The EORTC QLQ-CIPN20 subscales and questions did not differ between number of received cycles after adjustment for time between first and last cycle of oxaliplatin and cumulative dose. Moreover, dose intensity did not contribute to the EORTC QLQCIPN20 subscales and questions (data not shown). In addition, there was no difference in mean sensory scale score between patients who did $(n=144)$ or did not $(n=56)$ receive a dose reduction ( 15 vs. $17 ; p=0.61$ ). However, patients who received a dose 
reduction after the sixth cycle of oxaliplatin $(n=35)$ reported more severe neuropathy symptoms than patients who received a dose reduction earlier $(n=90)$, resulting in a worse mean sensory scale (mean 25 vs. $11 ; p=0.001$ ).

There was a trend of worse mean sensory scores in patients with documented acute neuropathy ( $n=155,75 \%$ ) when comparing patients who received a higher cumulative dose $(n=55)$ to lower cumulative dose $(n=11)$ (mean 20 vs. 7, $p=0.09$ ). Moreover, patients with documented acute neuropathy during treatment who received a dose reduction because of this neuropathy $(n=50)$ still reported significantly worse scores on the sensory scale at very similar cumulative doses, than those who did not receive a dose reduction because of neuropathy ( $n=96)$ (mean 21 vs. 15; $p=0.01$ ).

There were no significant differences between those treated with CAPOX $(n=173)$ or FOLFOX ( $n=27)$. Patients who received a higher cumulative dose of oxaliplatin in combination with capecitabine had worse EORTC QLQ-CIPN20 sensory scores than those who received a low cumulative dose (mean 18 vs. 9; $p=0.06$ ). Patients treated with FOLFOX reported similar results (mean 20 vs. 7; $p=0.31$ ). No differences in the other EORTC QLQ-CIPN20 subscales were observed in the subgroup analysis. Furthermore, patients treated with CAPOX who received a higher cumulative dose reported significantly more tingling toes or feet in comparison with low cumulative dose (14 vs. $1 \% ; p=0.01$ ). FOLFOX-treated patient reported similar results in tingling toes and feet (12 vs. $4 \%$ ).

Moreover, overall there were no differences between categories of age at time of diagnosis (data not shown). In addition, recurrence of disease between patients who received a low or a high cumulative dose did not differ (data not shown).

\section{Discussion}

Median four years after diagnosis, neuropathy symptoms are still frequently reported by CRC patients who underwent adjuvant treatment with oxaliplatin. Symptoms were mainly sensory and consisted of tingling toes or feet (30\%), numbness in toes or feet (19\%), tingling hands or fingers (15\%) and burning or shooting pain in the toes or feet (13\%). The actually delivered cumulative dose of oxaliplatin was associated with CIPN, and especially sensory complaints in toes and feet were significantly more often reported in patients who received a higher cumulative dose. Dose intensity did not contribute to severity of long-term CIPN. Therefore, decreasing the total cumulative dose is supposed to reduce long-term neuropathy, whereas delaying chemotherapy cycles probably is not beneficial. Patients who received a dose reduction because of neuropathy during oxaliplatin treatment, at very similar cumulative doses, still reported more long-term neuropathy symptoms. Furthermore, dose reduction after the sixth cycle of oxaliplatin was associated with more severe neuropathy symptoms. 
Therefore, it is important to decrease cumulative dose in patients with presenting neuropathy as soon as possible.

Since the introduction of CRC screening, more patients are faced with early stage CRC. Adjuvant treatment with oxaliplatin in combination with 5-FU/LV or capecitabine, showed an absolute risk reduction of approximately $5 \%$ on disease-free and overall survival, ${ }^{1,17}$ and became standard care for stage II and III CRC. ${ }^{14}$ Accordingly, more CRC survivors are confronted with the long-term side effects of oxaliplatin, in particular CIPN, which can have a substantial impact on patient's quality of life. ${ }^{2}$ The MOSAIC and NSABP C-07 trials reported the development of CIPN after four years and 27 months respectively in $15 \%$ and $10 \%$ of patients after a mean cumulative dose of $810 \mathrm{mg} / \mathrm{m}^{2}$ and $667 \mathrm{mg} / \mathrm{m}^{2}{ }^{2}{ }^{14,15}$ However, CIPN was not a primary endpoint and no association with different cumulative doses was reported. A recent review demonstrated that only few studies directly investigated the influence of cumulative dose on persistent CIPN more than a year after treatment. ${ }^{11}$ This review reports that two retrospective studies in CRC patients found that CIPN was associated with a median cumulative dose of $\geq 834$ and $850 \mathrm{mg} / \mathrm{m}^{2}$, respectively, and four other studies found similar results. Our results are similar, as patients who received more than $842 \mathrm{mg} / \mathrm{m}^{2}$ had more severe CIPN than patients who received less oxaliplatin. Therefore the results from the International Duration Evaluation of Adjuvant Chemotherapy (IDEA) and the TOSCA non-inferiority studies, which compare diseasefree survival between standard six vs. alternative three months (planned cumulative dose of $1030 \mathrm{mg} / \mathrm{m}^{2}$ vs. $520 \mathrm{mg} / \mathrm{m}^{2}$ oxaliplatin respectively) of adjuvant treatment for CRC with oxaliplatin, are important. ${ }^{18,19}$ These studies will prospectively demonstrate the optimal number of adjuvant courses with oxaliplatin in relation to both effectiveness and toxicity and might justify treatment with only six cycles of FOLFOX or four cycles of CAPOX with lower associated cumulative doses of oxaliplatin, which is supposed to result in a reduction of persistent CIPN.

Unlike other studies, the majority of patients included in this study were treated with CAPOX instead of FOLFOX. ${ }^{11}$ Studies investigating CIPN in patients treated with CAPOX are scarce. However, type of combination therapy may influence the degree of neuropathy. One study reported that FOLFOX was associated with increased incidence of CIPN compared with CAPOX at a similar cumulative dose. ${ }^{20}$ Another study reported more grade $\geq 3$ neuropathy ( $26 \%$ vs. $11 \%$; $p<0.001$ ) in patients treated with FOLFOX vs. CAPOX. ${ }^{21}$ In our study, EORTC QLQ-CIPN20 subscales were similar between regimens. However, patients were mainly treated with CAPOX and a minority with FOLFOX, which makes drawing conclusions on CIPN due to FOLOX difficult.

Acute neuropathy is unlikely to be caused by structural damage of axons and is more likely to be caused by changes in voltage-dependent sodium and/or potassiumchannels in comparison with persistent CIPN. $^{4,7}$ However, patients with acute neuropathy and those who received a dose reduction because of acute neuropathy also had complaints of long-term CIPN. This suggests that acute neurotoxicity is 
associated with the development of persistent CIPN. A possible explanation might be that the changes in the ion-channels lead to axonal damage. This is in concordance with the hypothesis that following to changes in $\mathrm{Nat-channels,} \mathrm{calcium} \mathrm{enters} \mathrm{and}$ accumulates in the axons leading to destruction, irreversible damage and therefore persistent CIPN. ${ }^{22}$ Furthermore, recently single nucleotide polymorphisms of voltagegated $\mathrm{Nat-channels} \mathrm{genes} \mathrm{or} \mathrm{oxaliplatin} \mathrm{metabolism} \mathrm{were} \mathrm{associated} \mathrm{with} \mathrm{an}$ increased incidence of oxaliplatin-induced neurotoxicity. ${ }^{5,6,23}$ In addition, knockout of SCN9a, which encodes for the $\mathrm{Na}_{v}$ 1.7-channel, demonstrated to cause anosmia in mice $^{24}$ and loss of smelling during oxaliplatin treatment might thus predispose development of CIPN. Nonetheless, loss of smelling is not an item in the most commonly used EORTC QLQ-CIPN20 questionnaire. Discovering specific neuropathy symptoms that predispose the development of CIPN would be of great interest.

We were able to collect data regarding cumulative dose, oxaliplatin administration and acute neuropathy during treatment for most of the patients. However, the retrospective collection of this data is a limitation of the study. The $\mathrm{NCl}-\mathrm{CTC}$ was lacking in more than $50 \%$ of patients and acute neuropathy was defined as having any documented neuropathy symptoms during treatment. Moreover, we had no information about the presence of neuropathy symptoms before treatment with oxaliplatin. Furthermore, data on possible recurrence of disease with complementary chemotherapy within the years after diagnosis was lacking for patients that did not initially receive oxaliplatin. In addition, we have adjusted the analysis for the presence of DM and other co-morbidities. However, selection bias could have occurred because patients with DM are less often treated with chemotherapy compared to patients without DM. ${ }^{25}$

We believe that our study contributes to the knowledge of persistent CIPN after adjuvant treatment with oxaliplatin, with the majority of patients treated with CAPOX, especially since there is no proven therapy to treat CIPN. Our study demonstrates that a higher cumulative dose is associated with the development of long-term CIPN. Monitoring symptoms of neuropathy during treatment is important as the risk of developing persistent CIPN may only be reduced by decreasing the cumulative dose of oxaliplatin on time, whereas delaying chemotherapy cycles probably is not beneficial. In addition, clinicians should be aware that acute neuropathy seems to be associated with long-term CIPN and that patients, in spite of receiving a dose reduction because of acute neuropathy, are still at risk of developing long-term CIPN. Patients should be informed on this matter. Future studies should focus on identifying patients with an increased risk of developing CIPN. In that way, cancer treatment might become more tailored as physicians might decide to restrain oxaliplatin treatment for certain patients. 


\section{References}

1. Andre T, Boni C, Mounedji-Boudiaf L, Navarro M, Tabernero J, Hickish T et al. Oxaliplatin, fluorouracil, and leucovorin as adjuvant treatment for colon cancer. N Engl J Med 2004;350:2343-2351.

2. Mols F, Beijers T, Lemmens V, van den Hurk CJ, Vreugdenhil G, van de Poll-Franse LV. Chemotherapyinduced neuropathy and its association with quality of life among 2- to 11-year colorectal cancer survivors: results from the population-based PROFILES registry. J Clin Oncol 2013;31:2699-707.

3. Mols F, Beijers T, Vreugdenhil G, van de Poll-Franse L. Chemotherapy-induced peripheral neuropathy and its association with quality of life: a systematic review. Support Care Cancer 2014;22:2261-2269.

4. Park SB, Lin CS, Krishnan AV, Goldstein D, Friedlander ML, Kiernan MC. Dose effects of oxaliplatin on persistent and transient $\mathrm{Na}+$ conductances and the development of neurotoxicity. PLoS One 2011;6: e18469.

5. Faber CG, Hoeijmakers JG, Ahn HS, Cheng X, Han C, Choi JS et al. Gain of function Nanu1.7 mutations in idiopathic small fiber neuropathy. Ann Neurol 2012;71:26-39.

6. Faber CG, Lauria G, Merkies IS, Cheng X, Han C, Ahn HS et al. Gain-of-function Nav1.8 mutations in painful neuropathy. Proc Natl Acad Sci U S A 2012;109:19444-19449.

7. Adelsberger H, Quasthoff S, Grosskreutz J, Lepier A, Eckel F, Lersch C. The chemotherapeutic oxaliplatin alters voltage-gated $\mathrm{Na}(+)$ channel kinetics on rat sensory neurons. Eur J Pharmacol 2000; 406:25-32.

8. Matsumoto S, Nishimura T, Kanai M, Mori Y, Nagayama S, Kawamura J et al. Safety and efficacy of modified FOLFOX6 for treatment of metastatic or locally advanced colorectal cancer. A singleinstitution outcome study. Chemotherapy 2008;54:395-403.

9. Park SB, Lin CS, Krishnan AV, Goldstein D, Friedlander ML, Kiernan MC. Long-term neuropathy after oxaliplatin treatment: challenging the dictum of reversibility. Oncologist 2011;16:708-716.

10. Cavaletti G, Tredici G, Petruccioli MG, Donde E, Tredici P, Marmiroli P et al. Effects of different schedules of oxaliplatin treatment on the peripheral nervous system of the rat. Eur J Cancer 2001;37: 2457-2563.

11. Beijers AJ, Mols F, Vreugdenhil G. A systematic review on chronic oxaliplatin-induced peripheral neuropathy and the relation with oxaliplatin administration. Support Care Cancer 2014;22: 1999-2007.

12. van de Poll-Franse LV, Horevoorts N, van Eenbergen M, Denollet J, Roukema JA, Aaronson NK et al. The Patient Reported Outcomes Following Initial treatment and Long term Evaluation of Survivorship registry: scope, rationale and design of an infrastructure for the study of physical and psychosocial outcomes in cancer survivorship cohorts. Eur J Cancer 2011;47:2188-2194.

13. Postma TJ, Aaronson NK, Heimans JJ, Muller MJ, Hildebrand JG, Delattre JY et al. The development of an EORTC quality of life questionnaire to assess chemotherapy-induced peripheral neuropathy: the QLQ-CIPN20. Eur J Cancer 2005;41:1135-1139.

14. Andre T, Boni C, Navarro M, Tabernero J, Hickish T, Topham C et al. Improved overall survival with oxaliplatin, fluorouracil, and leucovorin as adjuvant treatment in stage II or III colon cancer in the MOSAIC trial. J Clin Oncol 2009;27:3109-3116.

15. Land SR, Kopec JA, Cecchini RS, Ganz PA, Wieand HS, Colangelo LH et al. Neurotoxicity from oxaliplatin combined with weekly bolus fluorouracil and leucovorin as surgical adjuvant chemotherapy for stage II and III colon cancer: NSABP C-07. J Clin Oncol 2007;25:2205-2211.

16. Storey DJ, Sakala M, McLean CM, Phillips HA, Dawson LK, Wall LR et al. Capecitabine combined with oxaliplatin (CapOx) in clinical practice: how significant is peripheral neuropathy? Ann Oncol 2010;21: 1657-1661.

17. Schmoll HJ, Cartwright T, Tabernero J, Nowacki MP, Figer A, Maroun J et al. Phase III trial of capecitabine plus oxaliplatin as adjuvant therapy for stage III colon cancer: a planned safety analysis in 1,864 patients. J Clin Oncol 2007;25:102-109. 
18. Andre T, Iveson T, Labianca R, Meyerhardt JA, Souglakos I, Yoshino T et al. The IDEA (International Duration Evaluation of Adjuvant Chemotherapy) Collaboration: Prospective Combined Analysis of Phase III Trials Investigating Duration of Adjuvant Therapy with the FOLFOX (FOLFOX4 or Modified FOLFOX6) or XELOX (3 versus 6 months) Regimen for Patients with Stage III Colon Cancer: Trial Design and Current Status. Curr Colorectal Cancer Rep 2013;9:261-269.

19. Labianca R. FOLFOX-4 3months Versus 6 Months and Bevacizumab as Adjuvant Therapy for Patients With Stage II/III Colon Cancer (TOSCA). In. Gruppo Italiano per lo studio dei Carcinomi dell'Apparato Digerente 2014. (updated: September 3, 2013; cited July 22, 2014). Available from: http://www.clinicaltrials.gov/show/NCT00646607

20. Argyriou AA, Velasco R, Briani C, Cavaletti G, Bruna J, Alberti P et al. Peripheral neurotoxicity of oxaliplatin in combination with 5-fluorouracil (FOLFOX) or capecitabine (XELOX): a prospective evaluation of 150 colorectal cancer patients. Ann Oncol 2012;23:3116-3122.

21. Ducreux M, Bennouna J, Hebbar M, Ychou M, Lledo G, Conroy T et al. Capecitabine plus oxaliplatin (XELOX) versus 5-fluorouracil/leucovorin plus oxaliplatin (FOLFOX-6) as first-line treatment for metastatic colorectal cancer. Int J Cancer 2011;128:682-690.

22. LoPachin RM, Lehning EJ. Mechanism of calcium entry during axon injury and degeneration. Toxicol Appl Pharmacol 1997;143:233-244.

23. Argyriou AA, Cavaletti G, Antonacopoulou A, Genazzani AA, Briani C, Bruna J et al. Voltage-gated sodium channel polymorphisms play a pivotal role in the development of oxaliplatin-induced peripheral neurotoxicity: results from a prospective multicenter study. Cancer 2013;119:3570-3577.

24. Dib-Hajj SD, Yang Y, Black JA, Waxman SG. The Na(V)1.7 sodium channel: from molecule to man. Nat Rev Neurosci 2013;14:49-62.

25. Zanders MM, van Steenbergen LN, Haak HR, Rutten HJ, Pruijt JF, Poortmans PM et al. Diminishing differences in treatment between patients with colorectal cancer with and without diabetes: a population-based study. Diabet Med 2013;30:1181-1188. 


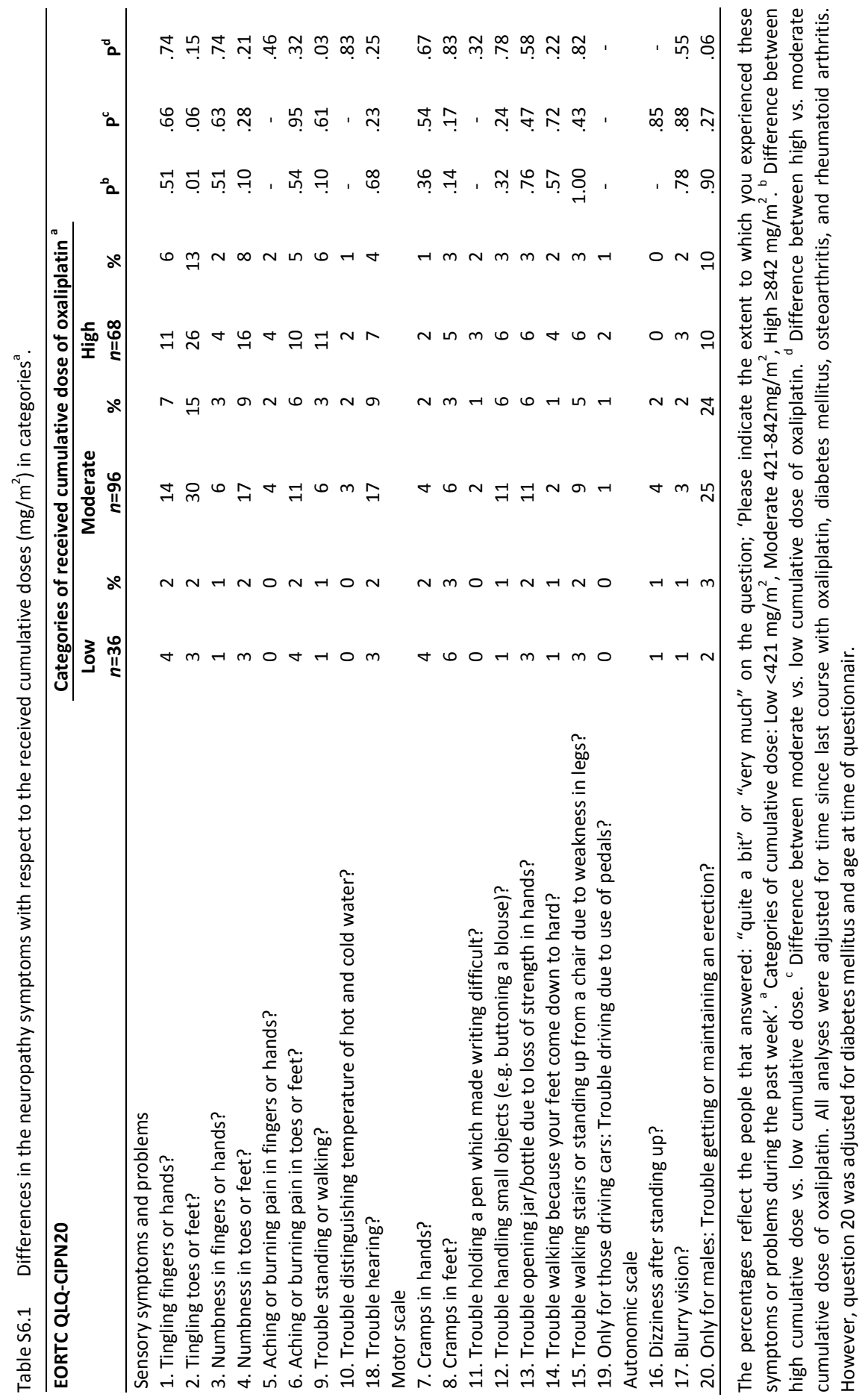




\section{Chapter}

The magnitude of neurotoxicity in patients with multiple myeloma and the impact of dose modifications: Results from the population-based PROFILES study

Beijers AJ, Oerlemans S, Mols F, Eurelings M, Minnema MC, Vreugdenhil G, van de Poll-Franse LV 


\section{Abstract}

Purpose

To assess (1) self-reported chemotherapy-induced peripheral neuropathy (CIPN) symptoms; (2) its association with sociodemographic and clinical characteristics; and (3) treatment dose modifications and its influence on the magnitude of neurotoxicity in a population-based cohort of patients with multiple myeloma (MM).

\section{Methods}

MM patients ( $n=156)$, diagnosed between 2000 and 2014, filled-out the EORTC QLQ-CIPN20 (65\% response). Data on treatment, outcomes, and dose modifications were extracted from the medical files.

\section{Results}

Fifty-three percent of patients reported at least one and on average three neuropathy symptoms that bothered them the most during the past week, with tingling toes/feet as most reported. In multivariate analysis, thalidomide, especially higher cumulative dose, was associated with neuropathy $(\beta=0.26, \mathrm{Cl} 95 \% 0.27-15.34, \mathrm{p}=0.04)$ and $\mathrm{CIPN}$ was not associated with age, sex, time since last course of therapy, number of prior therapies, osteoarthritis or diabetes. Dose modifications were often required (65\%). Although not statistically significant, a trend towards higher sensory ( 22 vs. 15 vs. $12, p=0.22$ ) and motoric neuropathy scores ( 21 vs. 15 vs. $11, p=0.36$ ) was observed among patients receiving dose modification because of CIPN (31\%) compared to those receiving a dose modification for another reason or no dose modification, without altering treatment response.

\section{Conclusion}

CIPN is a common dose limiting side effect in patients with MM. Severity of CIPN was mainly affected by treatment with thalidomide. In spite of dose modifications, patients still reported somewhat higher neuropathy scores without altered response rates. Early dose modification based on a more reliable tool for CIPN measurements may prove value. 


\section{Introduction}

The introduction of the immuno-modulatory drugs thalidomide and lenalidomide, and the proteasome inhibitor bortezomib, improved overall survival of patients with multiple myeloma (MM) in the past decade. ${ }^{1-3}$ Unfortunately, to achieve this improvement patients need treatment with several lines of therapy during the whole course of their disease. This treatment is associated with dose limiting neurotoxicity which is often referred to as chemotherapy-induced peripheral neuropathy (CIPN). ${ }^{2-4}$ Due to the increasing use of these neurotoxic agents, and since the treatment of CIPN remains unproven, ${ }^{5}$ more patients are confronted with CIPN, which has a negative influence on their quality of life. ${ }^{6}$

The neuropathy arises through neurotoxic agents that cause damage to nerve fibers, including the axon and/or dorsal root ganglion and presents predominantly with sensory symptoms and neuropathic pain, and sometimes leads to functional impairment or disability. ${ }^{2,7}$ It is assumed that most patients experience resolution or improvement of neurotoxicity after discontinuation or dose modification of the neurotoxic agents. However, this recovery may take months, and up to $25 \%$ of patients will experience persistent neurotoxicity. ${ }^{2}$ Nonetheless, as patients are often treated with several different neurotoxic agents during the whole course of their disease, resolution of symptoms remains uncertain, and little is known about the influence of sociodemographic and clinical characteristics on the magnitude of CIPN and the impact of dose modifications on CIPN. ${ }^{8}$ Recently we performed a populationbased cohort study in patients with $\mathrm{MM}^{6}{ }^{6}$ which reported a negative influence of CIPN on MM patients' quality of life. In that study, the development of a self-reported CIPN questionnaire was described which may prove to be of value in guiding dose modifications in daily practice. To get more insight into the influence of sociodemographic and clinical characteristics and the impact of dose modifications on CIPN we performed a secondary analysis.

The aims of this secondary analysis were (1) to assess the most frequently reported CIPN symptoms by patients themselves; (2) to determine the association between CIPN and sociodemographic and clinical characteristics, including treatment with different neurotoxic agents; and (3) to assess the amount of dose modifications and the influence of the dose modifications on the magnitude of CIPN in a populationbased cohort of patients with MM. 


\section{Methods}

\section{Setting and Participants}

A prospective population-based survey among patients with $\mathrm{MM}$ was conducted within the Netherlands Cancer Registry (NCR) with the goal to assess quality of life and disease specific complaints of patient with $\mathrm{MM}^{9}{ }^{9}$ The data presented in this secondary analysis are based upon the data collection wave of $2014^{6}$ which included a patient-reported CIPN questionnaire. The NCR was used to select all patients diagnosed with MM between 2000 and 2014 and registered in the Southeastern region of the NCR, which includes 10 hospitals. Data collection was accomplished within the Patient Reported Outcomes Following Initial treatment and Long-term Evaluation of Survivorship (PROFILES) registry. ${ }^{10}$ Patients who were younger than 18 years of age, were cognitively impaired, deceased prior to the study, or had an unknown address were excluded. Additionally, those who participated in the 2012 PROFILES survey and reported that they did no longer want to participate were excluded from this study. ${ }^{9,11}$ Detailed description of the data collection has been previously published. ${ }^{6,9,11}$ The certified Medical Ethics Committee of the Maxima Medical Center approved this study. All patients provided written informed consent.

\section{Sociodemographic and clinical characteristics}

Data on sociodemographic (sex, age) and clinical characteristics (including date of diagnosis, morphology, stage) were available from the NCR. The Population-based Haematological Registry for Observational Studies (PHAROS), which is an extension of the NCR, was used to complete these data with details of the received treatment (including type and date of treatment drug, number of received cycles, dose per cycle and applied dose modifications), and treatment outcomes. The data included within the NCR and PHAROS were collected by trained personnel from the medical files. Comorbidity at time of the study was assessed with the adapted Self-administered Comorbidity Questionnaire. ${ }^{12}$

\section{Patient-reported outcome chemotherapy-induced peripheral neuropathy}

CIPN was assessed with the validated Dutch version of the patient-reported European Organization for Research and Treatment of Cancer (EORTC) Quality of Life Questionnaire (QLQ)-CIPN20. ${ }^{13}$ The questionnaire contains twenty items which are divided into three subscales assessing sensory, motor, and autonomic symptoms, as well as a total CIPN score. Each item is measured on a Likert scale ranging from "not at all" (1) to "very much" (4). The achieved scores were transformed to a scale ranging from 0-100 and a higher score represented more CIPN complaints. 


\section{Statistical analysis}

Sociodemographic characteristics (e.g. sex, age) were compared between respondents and non-respondents, using Student's t-test for continuous data and $\mathrm{X}^{2}$ analysis for categorical data.

The prevalence of CIPN was defined as the percentage of patients who reported at least one neuropathy symptom that bothered them "quite a bit" or "very much. CIPN symptoms that disturbed patients the most during the past week were reported according to the EORTC QLQ-CIPN20 answer categories "quite a bit" or "very much" combined.

Multivariate linear regression models were used to analyze the association between sociodemographic (sex, age) and clinical characteristics (type of drug received, categories of time since the last cycle of received treatment and comorbidities osteoarthritis and diabetes mellitus that might interfere with general nervous system) with the EORTC QLQ-CIPN20 total score and subscales and the most important single questions. Type of treatment was defined as treatment with thalidomide 'yes' or ' $\mathrm{no}$ ', bortezomib 'yes' or 'no', lenalidomide 'yes' or 'no' and/or vincristine 'yes' or 'no'. Categories of time since last cycle of treatment were defined as last systemic treatment within the past 3 months, 3 to 12 months ago, and more than 12 months ago. Moreover, to determine the association of total number of received neurotoxic agents with CIPN, similar analysis were also done including total number of received neurotoxic agents $(0,1, \geq 2)$ instead of type of treatment. Variables were included into the model in separate steps. Demographic variables were added first, followed by clinical variables. In addition to the multivariate regression model, univariate analyses of the included characteristics were also reported.

Prevalence of patients who received a dose modification and the number of patients who received a dose modification due to neurotoxicity were presented in percentages and absolute numbers. EORTC QLQ-CIPN20 subscales were compared between patients in whom dose was modified due to another reason than CIPN, due to CIPN or did not receive a dose modification with analysis of covariance (ANCOVA). Confounding background variables were determined a priori ${ }^{14}$ and included time since last cycle of treatment, number of received neurotoxic agents, diabetes mellitus and osteoarthritis. Response rates (complete response; partial response/no change; relapsed/progressive disease combined) were compared between patients who received a dose modification or not with $\mathrm{X}^{2}$ analysis for categorical data.

All test were two-sided, considered significant if $p<0.05$, and were performed using SPSS version 22.0 (SPSS Inc. Chicago, IL, USA). 


\section{Results}

\section{Sociodemographic and clinical characteristics}

The questionnaire was completed by 156 patients with $\mathrm{MM}$ which entails a response rate of $65 \%$ ( 156 out of 241 eligible patients). Patients were mostly men ( $n=110,71 \%$ ) and on average 67.5 years of age at the time of the study. They were diagnosed with MM on average 3.3 years (SD 2.4) ago and most often had International Staging System (ISS) stage I or II disease (35\% and $27 \%$ respectively) (Table 7.1 ). Comorbidity was often reported, with the top three most mentioned comorbidities being back pain, osteoarthritis and hypertension. Comparison of respondents and nonrespondents showed that respondents were more often male ( $71 \%$ vs $47 \%, p<0.001$ ) and were significantly younger at the time of diagnosis (64.0 vs. 67.7 years old, $\mathrm{p}=0.004)$.

The majority of patients (92\%) were treated with systemic treatment, which was given in one $(50 \%)$ or several lines (36\%) of treatment (range $2-5)$. Median time since last course of systemic treatment was 10 months (range 0-158 months). Patients received treatment with one $(47 \%)$ or more $(43 \%)$ of the following neurotoxic drugs; bortezomib (51\%), thalidomide (47\%), lenalidomide (39\%) and/or vincristine (6\%) (Table 7.1). Patients who were only treated with one neurotoxic agent received mainly thalidomide (51\%; $n=37)$, followed by bortezomib $(32 \% ; n=23)$, lenalidomide $(12 \% ; n=9)$ and vincristine $(6 \% ; n=4)$.

\section{Prevalence and severity of CIPN}

In total, $92 \%$ of the responding patients reported at least one neuropathy symptom on the EORTC QLQ-CIPN20, and 53\% reported at least one, and on average three, neuropathy symptoms that bothered them "quite a bit" or "very much" in the past week. On a 100-point scale the total score on the EORTC QLQ-CIPN20 ranged from 0 to 74, with an averages score of 14.5 (SD 14.3). Subscales ranged from 0 to 95 and mean scores were 15.3 (SD 16.7, range 0-89), 15.0 (SD 17.7, range 0-95) and 13.0 (SD 18.4, range 0-83) for the sensory, motoric and autonomic scale, respectively. Neuropathy symptoms that were most often reported during the past week were mainly sensory complaints, i.e. tingling toes or feet (30\%), numbness in toes or feet (19\%) and tingling fingers or hands (17\%). The motoric symptom trouble opening jar/bottle due to loss of strength in hands (17\%), and the autonomic symptom trouble getting an erection (40\% of male patients) were also reported frequently (Figure 7.1). 
Table 7.1 Baseline sociodemographic and clinical characteristics of MM patients ( $n=156)$.

\begin{tabular}{|c|c|}
\hline & $\begin{array}{c}\text { MM patients } \\
n=156\end{array}$ \\
\hline \multicolumn{2}{|l|}{ Gender } \\
\hline Male & $110(71)$ \\
\hline Female & $46(30)$ \\
\hline Mean age at time of the survey in years (SD) & $67.5(9.3)$ \\
\hline \multicolumn{2}{|l|}{ Age at time of diagnosis, years $(n, \%)$} \\
\hline$<65$ years & $84(54)$ \\
\hline$>65$ years & $72(46)$ \\
\hline Mean years since diagnosis (SD) & $3.3(2.4)$ \\
\hline Years since diagnosis, (Range) & $0-13$ \\
\hline \multicolumn{2}{|l|}{ ISS stage at diagnosis, (n, \%) } \\
\hline 1 & $54(35)$ \\
\hline II & $42(27)$ \\
\hline III & $27(17)$ \\
\hline Unknown & $33(21)$ \\
\hline \multicolumn{2}{|l|}{ Physical performance status* (n, \%) } \\
\hline High & $32(21)$ \\
\hline Medium & $92(59)$ \\
\hline Low & $29(19)$ \\
\hline Unknown & $3(2)$ \\
\hline \multicolumn{2}{|l|}{ No. of comorbid conditions (n, \%) } \\
\hline None & $26(17)$ \\
\hline 1 & $33(21)$ \\
\hline $2+$ & $59(38)$ \\
\hline Unknown & $38(24)$ \\
\hline \multicolumn{2}{|l|}{ Most frequent conditions ( $n, \%)$} \\
\hline Back pain & $65(42)$ \\
\hline Osteoartritis & $49(31)$ \\
\hline Hypertension & $41(26)$ \\
\hline Anemia & $28(18)$ \\
\hline Heart disease & $29(19)$ \\
\hline Pulmonary disease & $16(10)$ \\
\hline Diabetes Mellitus & $11(7)$ \\
\hline \multicolumn{2}{|l|}{ Treatment received } \\
\hline \multicolumn{2}{|l|}{ Stem-cell transplant (n, \%) } \\
\hline Yes & $64(41)$ \\
\hline \multicolumn{2}{|l|}{ Systemic treatment (n, \%) } \\
\hline Yes & $143(92)$ \\
\hline No & $12(8)$ \\
\hline Unknown & $1(1)$ \\
\hline \multicolumn{2}{|l|}{ Total lines of treatment received $(n, \%)$} \\
\hline 0 & $12(8)$ \\
\hline 1 & $78(50)$ \\
\hline$\geq 2$ & $56(36)$ \\
\hline Unknown & $10(6)$ \\
\hline \multicolumn{2}{|l|}{ Received neurotoxic drug (n, \%) } \\
\hline Bortezomib & $80(51)$ \\
\hline Thalidomide & $73(47)$ \\
\hline Lenalidomide & $60(39)$ \\
\hline Vincristine & $9(6)$ \\
\hline
\end{tabular}


Table 7.1 (continued)

\begin{tabular}{lc}
\hline & $\begin{array}{c}\text { MM patients } \\
\mathbf{n = 1 5 6}\end{array}$ \\
\hline Cumulative dose received mg (mean, range) & $28997(200-88200)$ \\
Thalidomide & $49(9-146)$ \\
Bortezomib & $3940(250-13850)$ \\
Lenalidomide & $85-24)$ \\
Vincristine & \\
Amount of neurotoxic drugs received & $15(10)$ \\
0 & $73(47)$ \\
1 & $67(43)$ \\
$\geq 2$ & $1(1)$ \\
Unknown & $10(0-158)$ \\
Time since last therapy cycle (months) & \\
Median, (range) & $54(35)$ \\
Dose modification applied (n, \%) & $53(34)$ \\
No dose modification & $49(31)$ \\
Dose modification due to another reason than CIPN & \\
Dose modification because of CIPN & \\
\hline
\end{tabular}

CIPN: Chemotherapy-Induced Peripheral Neuropathy. Note: due to rounding off percentages can exceed 100\%. * Physical Performance: physical performance scale of the EORTC QLQ-C30 divided into 3 categories based on average and standard deviations derived from van de Poel et al, $2015^{11}$ : 1 SD under average physical functioning of MM patients is considered low physical functioning, whereas 1 SD above average MM patient physical functioning is considered high physical functioning.

\section{CIPN in relation to treatment}

In the univariate analysis, patients who developed more severe neuropathy were of higher age, were more recently treated with systemic therapy and received treatment with thalidomide (Table 7.2). Multivariate regression analysis showed that a higher age was associated with total neuropathy scores within block one (Table 7.2). However, after adding clinical characteristics to the model, the significance of age disappeared and treatment with thalidomide was the only independent factor which was associated with higher levels of neuropathy $(\beta=0.26, \mathrm{Cl} 95 \% 0.27-15.34, \mathrm{p}=0.04)$ (Table 7.2). Similar analysis were performed on the single questions and showed that treatment with thalidomide was mainly associated with sensory neuropathy with higher scores on numbness in toes or feet $(\beta=0.32, \mathrm{Cl} 95 \% 0.18-0.97, p=0.005)$, numbness in fingers or hands $(\beta=0.24, \mathrm{Cl} 95 \% 0.02-0.63, p=0.04)$ and higher scores on trouble holding a pen $(\beta=0.24, \mathrm{Cl} 95 \% 0.14-0.61, p=0.04)$. Within the group of patients treated with thalidomide $(n=73)$, a higher totally received cumulative dose of thalidomide was associated with more severe sensory neuropathy $(\beta=0.42, \mathrm{Cl} 95 \%$ $0.00-0.001, p=0.01$ ). In contrast, no association between treatment and motoric or autonomic neuropathy were found (data not shown). 
ஓे

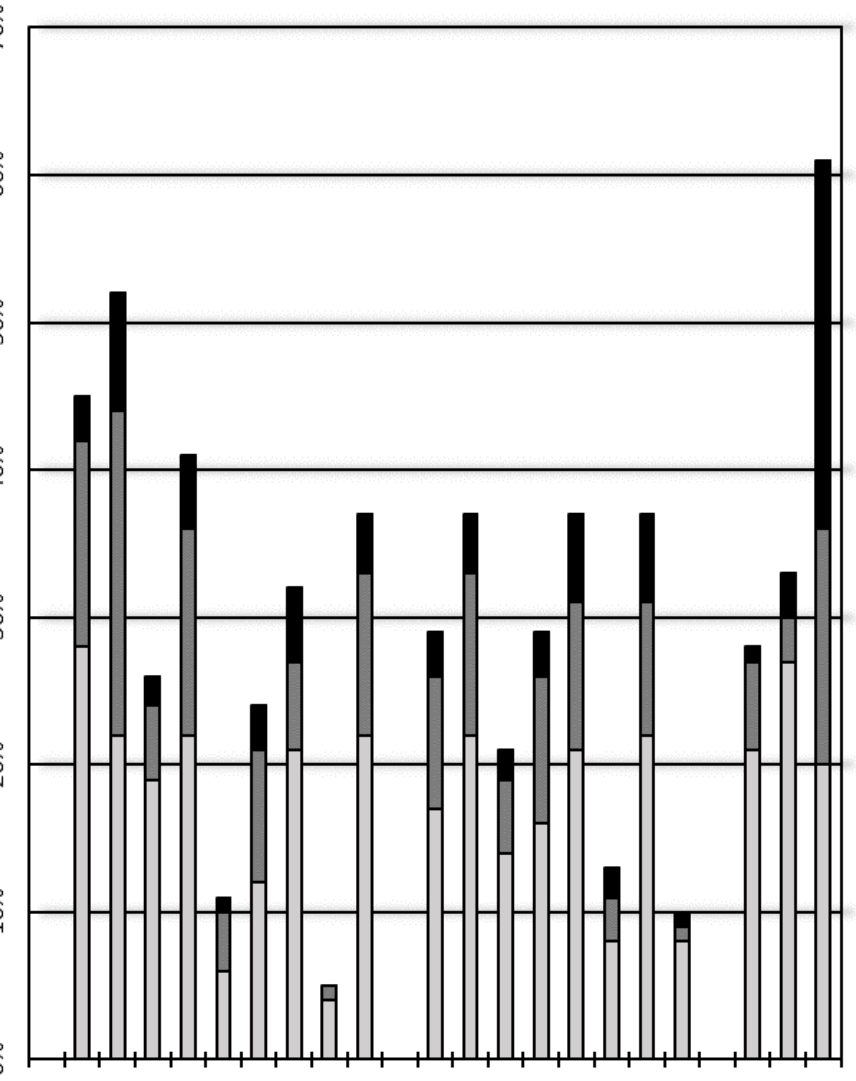

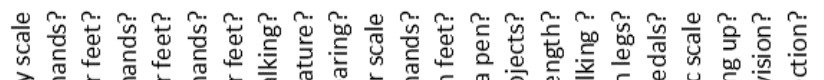

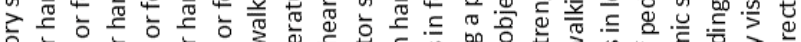

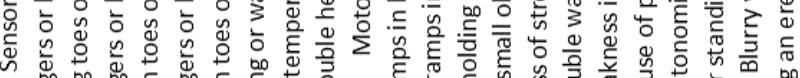

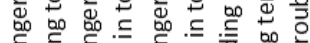

bo

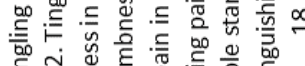

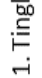

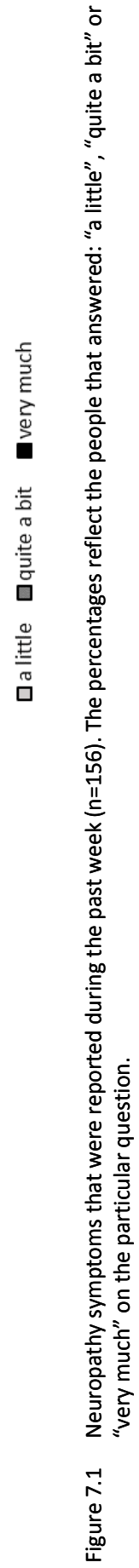


Multivariate regression analysis including the number of neurotoxic agents showed no difference in the EORTC QLQ-CIPN20 total and subscale scores between patients who received treatment with a combination of more than one neurotoxic agent $(n=73)$ compared to those treated with only one neurotoxic agent $(n=73)$ (mean score on the sensory subscale 18.3 vs. $14.9, p=0.47)$.

Table 7.2 Univariate and multivariate linear regression model of factors associated with neuropathy using the EORTC QLQ-CIPN20 total score.

\begin{tabular}{|c|c|c|c|c|c|c|}
\hline \multicolumn{7}{|l|}{ Variables } \\
\hline Demographic variables & 0.06 & $-3.36-7.42$ & 0.46 & & & \\
\hline \multicolumn{7}{|l|}{ Male vs. Female } \\
\hline Age & 0.17 & $0.01-0.51$ & 0.05 & & & \\
\hline \multicolumn{7}{|l|}{ Clinical variables } \\
\hline Osteoarthritis & 0.11 & $-2.25-8.72$ & 0.25 & & & \\
\hline Diabetes Mellitus & 0.08 & $-5.34-14.03$ & 0.38 & & & \\
\hline \multicolumn{7}{|l|}{ Time since last systemic } \\
\hline therapy & -0.12 & $-11.72-3.02$ & 0.24 & & & \\
\hline$<3$ months vs. $3-12$ months & -0.21 & $-12.12--0.46$ & 0.04 & & & \\
\hline \multicolumn{7}{|l|}{ <3months vs. $>12$ months } \\
\hline \multicolumn{7}{|l|}{ Received treatment with: } \\
\hline Thalidomide & 0.22 & $1.48-10.98$ & 0.01 & & & \\
\hline Bortezomib & 0.08 & $-2.62-7.08$ & 0.37 & & & \\
\hline Lenalidomide & 0.15 & $-0.65-9.22$ & 0.09 & & & \\
\hline Vincristine & -0.05 & $-12.63-6.90$ & 0.56 & & & \\
\hline \multirow{2}{*}{ Multivariate } & \multicolumn{3}{|c|}{ Model: Block 1} & \multicolumn{3}{|c|}{ Model: Block $1+2$} \\
\hline & $\boldsymbol{\beta}$ & $95 \% \mathrm{Cl}$ & p-value & $\beta$ & $95 \% \mathrm{Cl}$ & p-value \\
\hline \multicolumn{7}{|c|}{ Block 1: Demographic variables } \\
\hline Male vs. Female & 0.08 & $-3.83-9.52$ & 0.39 & 0.08 & $-4.70-10.42$ & 0.45 \\
\hline Age & 0.22 & $0.05-0.66$ & 0.02 & 0.08 & $-0.23-0.49$ & 0.47 \\
\hline \multicolumn{7}{|l|}{ Block 2: Clinical variables } \\
\hline Osteoarthritis & & & & 0.10 & $-4.48-10.83$ & 0.41 \\
\hline Diabetes Mellitus & & & & 0.09 & $-6.53-17.49$ & 0.37 \\
\hline \multicolumn{7}{|l|}{ Time since last systemic } \\
\hline therapy & & & & -0.01 & $-11.37-7.85$ & 0.72 \\
\hline$<3$ months vs. $3-12$ months & & & & -0.19 & $-12.86-3.58$ & 0.27 \\
\hline \multicolumn{7}{|l|}{$<3$ months vs. $>12$ months } \\
\hline \multicolumn{7}{|l|}{ Received treatment with: } \\
\hline Thalidomide & & & & 0.26 & $0.27-15.34$ & 0.04 \\
\hline Bortezomib & & & & 0.05 & $-5.84-8.78$ & 0.69 \\
\hline Lenalidomide & & & & 0.14 & $-3.25-11.95$ & 0.26 \\
\hline Vincristine & & & & -0.20 & $-12.87-3.58$ & 0.85 \\
\hline
\end{tabular}

$\mathrm{Cl}$ : confidence interval; EORTC QLQ-CIPN20: European Organisation for Research and Treatment of Cancer Quality of Life Questionnaire Chemotherapy-Induced Peripheral Neuropathy 20. 


\section{The impact of dose modifications on CIPN}

Dose was modified in $65 \%(n=102)$ of patients who received systemic treatment. In $31 \%(n=49)$ of patients, CIPN was described in the medical file to be the reason for dose modification. There were no differences in neuropathy scores between patients who received a dose modification during treatment and those who did not receive a dose modification. However, patients who received a dose modification because of CIPN ( $n=49)$ showed a trend towards worse total neuropathy (20 vs. 14 vs. 10, $\mathrm{p}=0.29$ ), sensory ( 22 vs. 15 vs. $12, \mathrm{p}=0.22$ ) and motoric neuropathy ( 21 vs. 15 vs. 11 , $\mathrm{p}=0.36)$ compared to patients who received a dose modification for another reason $(n=53)$ or no dose modification $(n=41)$ (Figure 7.2). No differences were observed for autonomic symptoms.

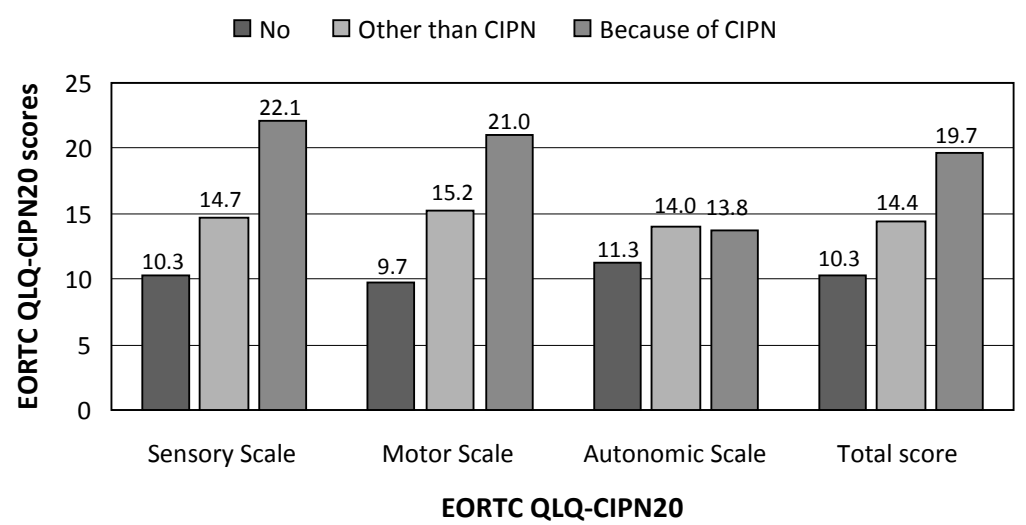

Figure 7.2 Comparison of EORTC QLQ-CIPN20 subscales according to categories of dose moditication. ${ }^{\mathrm{a}}$ CIPN: Chemotherapy-induce peripheral neuropathy; EORTC QLQ-CIPN20: European Organisation for Research and Treatment of Cancer Quality of Life Questionnaire Chemotherapy-Induced Peripheral Neuropathy 20. ${ }^{a}$ Categories of dose modification: 'no dose modification applied'; 'dose modification applied because of another reason than CIPN'; 'dose modification applied because of CIPN'. Standard deviations according to categories of applied dose modification; Sensory Scale: 11.9, 19.3, 16.5 respectively; Motor Scale: 11.9, 19.9, 19.1 respectively; Autonomic Scale: 17.3, 17.3, 20.9; Total Score 10.3, 17.0, 13.6 respectively.

In spite of the often applied dose modifications, patients were still treated with neurotoxic drugs during almost one third (28\%) of the time between diagnosis and the survey. This period of active treatment was received in several lines of treatment in more than a third (36\%) of the patients. Treatment response rates were available in $81 \%(n=126 / 156)$ of patients. Comparison of distribution of response rates complete response vs. partial response or no change vs. relapsed/progressive disease were not significantly different between patients who received a dose modification compared 
to those who did not receive a dose modification. Therefore applied dose modification to the treatment did not appear to adversely affect response rates (Figure 7.3).

$\mathrm{p}=0.632$

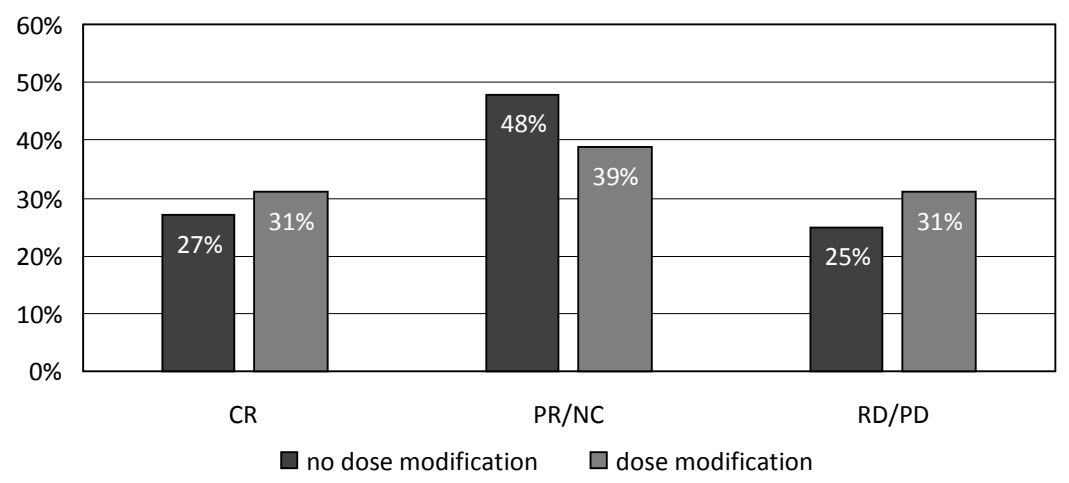

Figure 7.3 Comparison in distribution of treatment response rates ${ }^{\mathrm{a}}(\%)$ within patients who did not or did receive a dose modification. Response rates ${ }^{\mathrm{a}}: \mathrm{CR}=$ complete response; $\mathrm{PR} / \mathrm{NC}=$ partial response/no change combined; $R D / P D=$ relapsed/progressive disease combined. Note: due to rounding off percentages can exceed $100 \%$.

\section{Discussion}

This population-based study showed that considerable neuropathy was reported by more than half of MM patients, 0-13 years after diagnosis. Dose modifications were required in 7 out of 10 patients, whereas in almost one third of the patients CIPN was the main motive to decide on dose modification. Unfortunately, in spite of the dose alterations patients reported a trend towards worse total, sensory and motoric neuropathy compared to those who did not require a dose modification due to CIPN. The reported symptoms were mainly sensory and consisted of tingling and/or numbness in the feet and hands or loss of strength in the hands. It might be expected that these symptoms are age- or comorbidity related, caused by the disease itself ${ }^{15,16}$ or diminish after one year; however, this study showed otherwise and only treatment with thalidomide, and especially a higher cumulative dose of thalidomide, was associated with neuropathy. Time since last course of therapy was not significantly associated with the magnitude of CIPN and therefore many patients remain confronted with this toxicity during the whole period of their disease. Since no therapy has proven to be effective in the treatment of CIPN, prevention of this toxicity is necessary. 
Dose modifications did not seem to alter response rates. Consequently, it could be argued that dose modifications could be safely applied at lower levels of reported CIPN than now is recommended as still many patients in this study are confronted with persistent CIPN. Nowadays, the National Cancer Institute-Common Toxicity Criteria for adverse events (NCI-CTCAE) are the foundation of the dose modification guidelines. However, clinicians have trouble distinguishing those criteria from each other and CIPN is often underreported by clinicians. ${ }^{17,18}$ Therefore dose modifications may mistakenly be applied at higher levels of CIPN than they should according to the guidelines. More accurate monitoring of CIPN may be helpful in this regard. We recently proposed a self-reported questionnaire which may prove to be of value in monitoring the course of CIPN and guiding dose modification decisions. ${ }^{6}$ This selfreported questionnaire was developed to give an accurate and uniform indication regarding the level of CIPN, and overcomes underreporting of CIPN by clinicians. Other potentially interesting ways of assessing CIPN in this way are the patientreported outcome (PRO)-CTCAE criteria in which PRO measurements are integrated in $\mathrm{NCl}$-CTCAE reporting. ${ }^{19,20}$ A prospective trial should be initiated to investigate whether a self-reported questionnaire is able to guide dose modifications, and if dose modifications could be safely applied at lower levels of CIPN without compromising response rates.

Furthermore, patients were actively treated with neurotoxic drugs for almost a third of the period between diagnosis and the time of survey. This period of active treatment was received in several lines of treatment in $36 \%$ of patients, implying short treatment free intervals between regimens. As those drugs are accompanied with many adverse events including CIPN, this means that patients are constantly confronted with the burden of accumulative side effects, which has a negative influence on their quality of life. ${ }^{6,21}$ Although the overall survival of patients has improved in the last decade, cure of $\mathrm{MM}$ is still very scarce. The burden of the treatment should be placed into the benefits of therapy. ${ }^{22}$

Treatment with thalidomide predicted more severe neuropathy, than treatment with other neurotoxic agents. Thalidomide in combination with melphalan/prednisone was considered standard treatment of $\mathrm{MM}$ treatment in the past decades ${ }^{23}$ and consequently was often used in the included population. However, in the last years treatment of MM has considerably changed and bortezomib nowadays has become frontline therapy and more recently lenalidomide can be used. ${ }^{24}$ Treatment with bortezomib is associated with CIPN as a frequent and dose limiting adverse event. ${ }^{25,26}$ However, it has diminished since a phase 3 study showed that subcutaneous administration is accompanied with lower levels of grade 2 or worse (24\%) neurotoxicity compared to intravenous administration (41\%) of bortezomib without impairing response rates. ${ }^{27}$ Consequently, subcutaneous administration has become first choice. Although encouraging, treatment with subcutaneous bortezomib is still accompanied by all grade neurotoxicity in $38 \%$ of patients. ${ }^{27}$ Therefore, great efforts 
have been made to investigate next generation proteasome inhibitors such as carfilzomib and ixazomib, which express lower grades of neuropathy. ${ }^{28-30}$ Since treatment of CIPN remains unproven and next generation proteasome inhibitors are still under investigation, neurotoxicity continues to be a challenging and major dose limiting adverse event of anti-myeloma treatment. Consequently, prevention and accurate monitoring of CIPN in order to diminish the burden of this side effect remains important.

Comparison with other population-based studies investigating CIPN in patients with $\mathrm{MM}$ is rather difficult since most performed studies reporting on CIPN were randomized clinical trials (RCTs) including a selected group of patients. CIPN assessment in studies who did include patients outside of clinical trials is diverse, but is mainly described according to the NCI-CTCAE or the EORTC QLQ-Myeloma Module (MY20), and more informative patient-reported measurements, as the EORTC QLQCIPN20, are not often used. For example, a small cross-sectional study $(n=32)$ reported tingling hands/feet, according to the EORTC QLQ-MY20, in 53\% of patients as the most common neuropathy symptom. ${ }^{31}$ Moreover, a German multicenter crosssectional study $(n=154)$ used a non-validated adverse event checklist and reported hypoesthesia or paresthesia (33\%), muscle cramps (31\%), and sensory neuropathy $(15 \%)$ in patients with $\mathrm{MM}^{21}$ A patient-reported outcome was used in two sub analysis of large trials ${ }^{25,26}$ investigating the association of bortezomib and CIPN. Most reported items included tingling hands/feet, numbness in hands/feet, trouble buttoning buttons, and trouble feeling the shape of small objects in the hand.

This is a population-based study that included a large population of patients with MM with a good inquiry response. Consequently, the results of this study could be extrapolated to the general population with MM. CIPN was measured with a selfreported questionnaire, which revealed more detailed information regarding experienced neuropathy symptoms in patients with MM compared to the CTC-grading scales mainly used in clinical trials. Nevertheless, the study also has some restrictions. The response rate was good, but it is unknown whether non-respondents did not complete the survey because of a lower performance status due to the disease, neuropathy symptoms to the hands or due to lack of symptoms. Selection bias may have occurred since deceased patients and patients who refused to participate after the 2012 survey were excluded. In addition, respondents were more often male, although also the disease has an unequal divided sex ratio with more males. In addition, we did not have information on the presence of neuropathy before the start of treatment. However, we did adjust for diabetes mellitus and osteoarthritis, which can cause neuropathy-like symptoms. Moreover, we did not have information about the method of bortezomib administration (intravenously of subcutaneously), which may have influenced the association between bortezomib and neuropathy. Due to our cross-sectional design, we were not able to determine if the neuropathy symptoms resolved after dose modification. Furthermore, dose modifications did not reveal to 
affect response rates. However, study design and patient numbers in each group are too small to draw definitive conclusions. In addition, we do not know why certain treatment decisions had been made which may have influenced the degree of neuropathy.

CIPN is a common dose limiting side effect reported by more than half of the overall non-selected patients with MM. Severity of CIPN was mainly affected by treatment with thalidomide, and was not associated with age, sex, time since last course of therapy, number of prior therapies and comorbidity. Especially a higher cumulative dose of thalidomide was associated with higher levels of neuropathy. Since no therapy has proven to be effective in the treatment of CIPN, prevention of this toxicity is necessary. Dose modification seemed not to alter response rates. Therefore, early dose modification based on a more reliable tool for CIPN measurements seems warranted. Future prospective trials investigating the use of self-reported questionnaires to guide dose modification, and dose modifications at lower levels of CIPN without compromising response rates should be initiated.

\section{Acknowledgement}

The study was supported by Janssen-Cilag pharmaceutical companies of JohnsonJohnson. 


\section{References}

1. Cavo M, Rajkumar SV, Palumbo A, Moreau P, Orlowski R, Blade J, Sezer O, Ludwig H, Dimopoulos MA, Attal M, Sonneveld P, Boccadoro M, Anderson KC, Richardson PG, Bensinger W, Johnsen HE, Kroeger N, Gahrton G, Bergsagel PL, Vesole DH, Einsele H, Jagannath S, Niesvizky R, Durie BG, San Miguel J, Lonial S, International Myeloma Working G. International Myeloma Working Group consensus approach to the treatment of multiple myeloma patients who are candidates for autologous stem cell transplantation. Blood 2011;117:6063-6073.

2. Delforge M, Blade J, Dimopoulos MA, Facon T, Kropff M, Ludwig H, Palumbo A, Van Damme P, SanMiguel JF, Sonneveld P. Treatment-related peripheral neuropathy in multiple myeloma: the challenge continues. Lancet Oncol 2010;11:1086-1095.

3. Kumar SK, Rajkumar SV, Dispenzieri A, Lacy MQ, Hayman SR, Buadi FK, Zeldenrust SR, Dingli D, Russell SJ, Lust JA, Greipp PR, Kyle RA, Gertz MA (2008) Improved survival in multiple myeloma and the impact of novel therapies. Blood 2008;111:2516-2520.

4. Beijers AJ, Jongen JL, Vreugdenhil G. Chemotherapy-induced neurotoxicity: the value of neuroprotective strategies. Neth J Med 2012;70:18-25

5. Hershman DL, Lacchetti C, Dworkin RH, Lavoie Smith EM, Bleeker J, Cavaletti G, Chauhan C, Gavin P, Lavino A, Lustberg MB, Paice J, Schneider B, Smith ML, Smith T, Terstriep S, Wagner-Johnston N, Bak K, Loprinzi CL (2014) Prevention and Management of Chemotherapy-Induced Peripheral Neuropathy in Survivors of Adult Cancers: American Society of Clinical Oncology Clinical Practice Guideline. J Clin Oncol 2014;32:1941-1967.

6. Beijers AJ, Vreugdenhil G, Oerlemans S, Eurelings M, Minnema MC, Eeltink CM, van de Poll-Franse LV, Mols F. Chemotherapy-induced neuropathy in multiple myeloma: influence on quality of life and development of a questionnaire to compose common toxicity criteria grading for use in daily clinical practice. Support Care Cancer 2016;24:2411-2420.

7. Jongen JL, Broijl A, Sonneveld P. Chemotherapy-induced peripheral neuropathies in hematological malignancies. J Neurooncol 2015;121:229-237.

8. Richardson PG, Delforge M, Beksac M, Wen P, Jongen JL, Sezer O, Terpos E, Munshi N, Palumbo A, Rajkumar SV, Harousseau JL, Moreau P, Avet-Loiseau H, Lee JH, Cavo M, Merlini G, Voorhees P, Chng WJ, Mazumder A, Usmani S, Einsele H, Comenzo R, Orlowski R, Vesole D, Lahuerta JJ, Niesvizky R, Siegel D, Mateos MV, Dimopoulos M, Lonial S, Jagannath S, Blade J, Miguel JS, Morgan G, Anderson KC, Durie BG, Sonneveld P. Management of treatment-emergent peripheral neuropathy in multiple myeloma. Leukemia 2012;26:595-608.

9. Mols F, Oerlemans S, Vos AH, Koster A, Verelst S, Sonneveld P, van de Poll-Franse LV. Health-related quality of life and disease-specific complaints among multiple myeloma patients up to $10 \mathrm{yr}$ after diagnosis: results from a population-based study using the PROFILES registry. Eur J Haematol 2012;89:311-319.

10. van de Poll-Franse LV, Horevoorts N, van Eenbergen M, Denollet J, Roukema JA, Aaronson NK, Vingerhoets A, Coebergh JW, de Vries J, Essink-Bot ML, Mols F, Profiles Registry G. The Patient Reported Outcomes Following Initial treatment and Long term Evaluation of Survivorship registry: scope, rationale and design of an infrastructure for the study of physical and psychosocial outcomes in cancer survivorship cohorts. Eur J Cancer 2011;47:2188-194.

11. van der Poel MW, Oerlemans S, Schouten HC, van de Poll-Franse LV. Elderly multiple myeloma patients experience less deterioration in health-related quality of life than younger patients compared to a normative population: a study from the population-based PROFILES registry. Ann Hematol 2015;94:651-661.

12. Sangha O, Stucki G, Liang MH, Fossel AH, Katz JN. The Self-Administered Comorbidity Questionnaire: a new method to assess comorbidity for clinical and health services research. Arthritis Rheum 2003;49:156-163. 
13. Postma TJ, Aaronson NK, Heimans JJ, Muller MJ, Hildebrand JG, Delattre JY, Hoang-Xuan K, LanteriMinet M, Grant R, Huddart R, Moynihan C, Maher J, Lucey R, Group EQoL. The development of an EORTC quality of life questionnaire to assess chemotherapy-induced peripheral neuropathy: the QLQCIPN20. Eur J Cancer 2005;41:1135-1139.

14. Babyak MA. What you see may not be what you get: a brief, nontechnical introduction to overfitting in regression-type models. Psychosom Med 2004;66:411-421.

15. Dimopoulos MA, Mateos MV, Richardson PG, Schlag R, Khuageva NK, Shpilberg O, Kropff M, Spicka I, Palumbo A, Wu KL, Esseltine DL, Liu K, Deraedt W, Cakana A, Van De Velde H, San Miguel JF (2011) Risk factors for, and reversibility of, peripheral neuropathy associated with bortezomib-melphalanprednisone in newly diagnosed patients with multiple myeloma: subanalysis of the phase 3 VISTA study. Eur J Haematol 2011;86 (1):23-31.

16. Park SB, Goldstein D, Krishnan AV, Lin CS, Friedlander ML, Cassidy J, Koltzenburg M, Kiernan MC. Chemotherapy-induced peripheral neurotoxicity: a critical analysis. CA Cancer J Clin 2013; 63 (6): 419-437.

17. Basch E, lasonos A, McDonough T, Barz A, Culkin A, Kris MG, Scher HI, Schrag D. Patient versus clinician symptom reporting using the National Cancer Institute Common Terminology Criteria for Adverse Events: results of a questionnaire-based study. Lancet Oncol 2006; 7 (11):903-909.

18. Di Maio M, Gallo C, Leighl NB, Piccirillo MC, Daniele G, Nuzzo F, Gridelli C, Gebbia V, Ciardiello F, De Placido S, Ceribelli A, Favaretto AG, de Matteis A, Feld R, Butts C, Bryce J, Signoriello S, Morabito A, Rocco G, Perrone F. Symptomatic toxicities experienced during anticancer treatment: Agreement between patient and physician reporting in three randomized trials. J Clin Oncol. 2015;33(8):910-915

19. Basch E, Reeve BB, Mitchell SA, Clauser SB, Minasian LM, Dueck AC, Mendoza TR, Hay J, Atkinson TM, Abernethy AP, Bruner DW, Cleeland CS, Sloan JA, Chilukuri R, Baumgartner P, Denicoff A, St Germain D, O'Mara AM, Chen A, Kelaghan J, Bennett AV, Sit L, Rogak L, Barz A, Paul DB, Schrag D. Development of the National Cancer Institute's patient-reported outcomes version of the common terminology criteria for adverse events (PRO-CTCAE). J Natl Cancer Inst 2014; 106 (9).

20. Dueck AC, Mendoza TR, Mitchell SA, Reeve BB, Castro KM, Rogak LJ, Atkinson TM, Bennett AV, Denicoff AM, O'Mara AM, Li Y, Clauser SB, Bryant DM, Bearden JD, 3rd, Gillis TA, Harness JK, Siegel RD, Paul DB, Cleeland CS, Schrag D, Sloan JA, Abernethy AP, Bruner DW, Minasian LM, Basch E, National Cancer Institute PROCSG. Validity and Reliability of the US National Cancer Institute's Patient-Reported Outcomes Version of the Common Terminology Criteria for Adverse Events (PROCTCAE). JAMA Oncol 2015; 1 (8):1051-1059.

21. Jordan K, Proskorovsky I, Lewis P, Ishak J, Payne K, Lordan N, Kyriakou C, Williams CD, Peters S, Davies FE. Effect of general symptom level, specific adverse events, treatment patterns, and patient characteristics on health-related quality of life in patients with multiple myeloma: results of a European, multicenter cohort study. Support Care Cancer 2014; 22 (2):417-426.

22. Beijers AJ, Mols F, van den Hurk CJ, Vreugdenhil A. Are chemotherapy-associated symptoms underestimated? A view beyond common toxicity criteria. Acta Oncol 2016;55 (4):516-518.

23. Fayers PM, Palumbo A, Hulin C, Waage A, Wijermans $P$, Beksac M, Bringhen $S$, Mary JY, Gimsing $P$, Termorshuizen F, Haznedar R, Caravita T, Moreau P, Turesson I, Musto P, Benboubker L, Schaafsma M, Sonneveld P, Facon T, Nordic Myeloma Study G, Italian Multiple Myeloma N, Turkish Myeloma Study G, Hemato-Oncologie voor Volwassenen N, Intergroupe Francophone du M, European Myeloma N. Thalidomide for previously untreated elderly patients with multiple myeloma: metaanalysis of 1685 individual patient data from 6 randomized clinical trials. Blood 2011; 118 (5): 1239-1247.

24. Benboubker L, Dimopoulos MA, Dispenzieri A, Catalano J, Belch AR, Cavo M, Pinto A, Weisel K, Ludwig H, Bahlis N, Banos A, Tiab M, Delforge M, Cavenagh J, Geraldes C, Lee JJ, Chen C, Oriol A, de la Rubia J, Qiu L, White DJ, Binder D, Anderson K, Fermand JP, Moreau P, Attal M, Knight R, Chen G, Van Oostendorp J, Jacques C, Ervin-Haynes A, Avet-Loiseau H, Hulin C, Facon T, Team FT. Lenalidomide and dexamethasone in transplant-ineligible patients with myeloma. N Engl J Med 2014; 371 (10): 906-917. 
25. Richardson PG, Briemberg H, Jagannath S, Wen PY, Barlogie B, Berenson J, Singhal S, Siegel DS, Irwin D, Schuster M, Srkalovic G, Alexanian R, Rajkumar SV, Limentani S, Alsina M, Orlowski RZ, Najarian K, Esseltine D, Anderson KC, Amato AA. Frequency, characteristics, and reversibility of peripheral neuropathy during treatment of advanced multiple myeloma with bortezomib. J Clin Oncol 2006;24 (19):3113-3120.

26. Richardson PG, Sonneveld P, Schuster MW, Stadtmauer EA, Facon T, Harousseau JL, Ben-Yehuda D, Lonial S, Goldschmidt H, Reece D, Blade J, Boccadoro M, Cavenagh JD, Boral AL, Esseltine DL, Wen PY, Amato AA, Anderson KC, San Miguel J. Reversibility of symptomatic peripheral neuropathy with bortezomib in the phase III APEX trial in relapsed multiple myeloma: impact of a dose-modification guideline. Br J Haematol 2009;144 (6):895-903.

27. Moreau P, Pylypenko H, Grosicki S, Karamanesht I, Leleu X, Grishunina M, Rekhtman G, Masliak Z, Robak T, Shubina A, Arnulf B, Kropff M, Cavet J, Esseltine DL, Feng H, Girgis S, van de Velde H, Deraedt W, Harousseau JL. Subcutaneous versus intravenous administration of bortezomib in patients with relapsed multiple myeloma: a randomised, phase 3, non-inferiority study. Lancet Oncol 2011;12 (5):431-440.

28. Dimopoulos MA, Moreau P, Palumbo A, Joshua D, Pour L, Hajek R, Facon T, Ludwig H, Oriol A, Goldschmidt H, Rosinol L, Straub J, Suvorov A, Araujo C, Rimashevskaya E, Pika T, Gaidano G, Weisel K, Goranova-Marinova V, Schwarer A, Minuk L, Masszi T, Karamanesht I, Offidani M, Hungria V, Spencer A, Orlowski RZ, Gillenwater HH, Mohamed N, Feng S, Chng WJ, investigators E. Carfilzomib and dexamethasone versus bortezomib and dexamethasone for patients with relapsed or refractory multiple myeloma (ENDEAVOR): a randomised, phase 3, open-label, multicentre study. Lancet Oncol 2016; 17 (1):27-38.

29. Kumar SK, Berdeja JG, Niesvizky R, Lonial S, Laubach JP, Hamadani M, Stewart AK, Hari P, Roy V, Vescio R, Kaufman JL, Berg D, Liao E, Di Bacco A, Estevam J, Gupta N, Hui AM, Rajkumar V, Richardson PG. Safety and tolerability of ixazomib, an oral proteasome inhibitor, in combination with lenalidomide and dexamethasone in patients with previously untreated multiple myeloma: an openlabel phase 1/2 study. Lancet Oncol 2014;15 (13):1503-1512.

30. Richardson PG, Moreau P, Laubach JP, Gupta N, Hui AM, Anderson KC, San Miguel JF, Kumar S. The investigational proteasome inhibitor ixazomib for the treatment of multiple myeloma. Future Oncol 2015; 11 (8):1153-1168.

31. Boland E, Eiser C, Ezaydi Y, Greenfield DM, Ahmedzai SH, Snowden JA. Living with advanced but stable multiple myeloma: a study of the symptom burden and cumulative effects of disease and intensive (hematopoietic stem cell transplant-based) treatment on health-related quality of life. J Pain Symptom Manage 2013; 46 (5):671-680. 


\section{Chapter 8}

Summary and general discussion 
Chapter 8 


\section{Summary of the results}

The main objectives of this thesis were to gain knowledge about the severity and prevalence of chemotherapy-induced peripheral neuropathy (CIPN), the influence of CIPN on patients' quality of life (QOL) and the influence of chemotherapy administration on the development of CIPN. This thesis was subdivided into two parts. Part one addressed the prevalence and severity of CIPN symptoms, and the impact of those symptoms on the QOL among different cancer patients, at different time points after diagnosis. Part two addressed the influence of chemotherapy administration on the development of persistent CIPN. We investigated the influence of oxaliplatin administration on persistent CIPN in colorectal cancer (CRC) survivors. In addition, the influence of antimyeloma treatment with immuno-modulatory drugs thalidomide and lenalidomide, and the proteasome inhibitor bortezomib on persistent CIPN in patients with multiple myeloma (MM) was reported.

\section{Part I - Chemotherapy-induced peripheral neuropathy and quality of life}

This thesis started with a study investigating the occurrence and severity of CIPN symptoms induced by the frequently used platinum derivatives and taxanes median six months after cessation of chemotherapy, as patients with remaining complaints after six months are at risk of having irreversible CIPN (Chapter 2). Furthermore, the impact of these symptoms on daily functioning and QOL was investigated. A sample of 43 cancer patients, including colorectal, breast-, ovarian and prostate cancer, visiting the outpatient clinic of the Máxima Medical Center were included. The majority of patients demonstrated the occurrence of CIPN symptoms in the upper and lower extremities. Overall, most reported complaints included numbness and tingling in hands as well as feet, suffering from cold feet, and trouble distinguishing objects in the hands. Housekeeping difficulties were reported in $13 \%$ of patients and $21 \%$ of patients became more dependent on others because of the neurotoxicity. In addition, the overall QOL was negatively affected due to the impact of CIPN in $49 \%$ of patients.

After concluding that CIPN was very common in patients with different cancer types visiting the outpatient clinic in chapter 2, we wanted to explore those symptoms in more detail in a population-based sample of CRC survivors long after diagnosis (Chapter 3). The platinum derivative oxaliplatin, first successfully used in the management of advanced CRC, is nowadays also the regimen of choice for adjuvant treatment of patients with curatively resected node-positive CRC, with improved survival rates as a consequence. Because of the increasing prevalence of CRC, the 
increased use of oxaliplatin, and because there is no therapy to treat CIPN, many patients keep being confronted with this debilitating side effect. Therefore, we investigated the prevalence and severity of CIPN, and its influence on QOL in a population-based sample of CRC survivors two to 11 years after diagnosis in chapter 3. The population-based Netherlands Cancer Registry (NCR) was used to select all CRC patients diagnosed between 2000 and 2009 in the Southeastern area of the Netherlands. Of the patients who were alive, $83 \%(n=1,643)$ of patients completed the European Organisation for Research and Treatment of Cancer Quality of Life Questionnaire (EORTC QLQ-C30) and its Chemotherapy-Induced Peripheral Neuropathy supplement (EORTC QLQ-CIPN20). Neuropathy-related symptoms were still often reported two to 11 years after diagnosis. Especially sensory symptoms in the lower extremities among those treated with oxaliplatin. Patients who received oxaliplatin more often reported tingling ( $29 \%$ vs. $8 \% ; p=0.001)$, numbness ( $17 \%$ vs. $5 \% ; p=0.005)$, and aching or burning pain ( $13 \%$ vs. $6 \% ; p=0.03)$ in toes or feet compared to those not treated with chemotherapy. They also more often reported tingling toes or feet $(29 \%$ vs. $14 \% ; p=0.01)$ compared with those treated with chemotherapy without oxaliplatin. In addition, those with many neuropathy symptoms (e.g. upper 10\%) reported statistically significant and clinically relevant worse QOL scores on all EORTC QLQ-C30 subscales (all $p<0.01$ ). In conclusion, this study showed that neuropathy symptoms are still often reported in CRC survivors two to 11 years after diagnosis, especially sensory symptoms among those treated with oxaliplatin. The neuropathy symptoms have a negative influence on patients' QOL.

Besides the effects of CIPN on the daily functioning and QOL among CRC survivors, we also studied these effects in patients with multiple myeloma (MM). Because of improved treatment and increased aging of the population, the prevalence of MM is increasing, and more patients are confronted with the side effects of its treatment. Currently dose modification or discontinuation in case of moderate symptoms of CIPN is the only way to prevent severe CIPN. Unfortunately, dose modifications can influence treatment response and therefore cautiousness is warranted. Many assessment tools for monitoring CIPN symptoms have been investigated, but they are not developed for use in clinical practice. In Chapter $\mathbf{4}$ we validated the self-reported Indication Common Toxicity Criteria (CTC)-grading Peripheral Neuropathy (ICPNQ) questionnaire, which can be used in daily clinical practice. Furthermore, we examined the prevalence of CIPN and its influence on QOL in MM patients. One-hundred fifty-six patients with $\mathrm{MM}$ were included (65\% response) on average 3.3 years after diagnosis. CIPN was often reported, and $65 \%$ of patients reported grades two or three neuropathy. The newly developed self-reported ICPNQ is a valid instrument to distinguish the highest CIPN CTC-grades from the lower CTC-grades in a populationbased cohort of MM patients. Hence, the self-reported ICPNQ provides in the need to give a standardized indication regarding the CTC-grades of CIPN, overcomes the issue 
of underreporting CIPN by clinicians, and can be incorporated easily in clinical practice. Patients with grade two with pain and grade three neuropathy combined reported statistically significant and clinically relevant worse QOL compared to patients who had neuropathy CTC-grades between zero to two without pain. This indicates that early dose modification based on a more reliable tool for CIPN measurements is needed.

\section{Part II - Chemotherapy-induced peripheral neuropathy and the influence of chemotherapy administration}

It is acknowledged that the degree of oxaliplatin-induced neuropathy (O-IPN) is dependent on cumulative dose, duration of administration and dose intensity. Nonetheless, this knowledge is mainly based on studies concerning the development of acute neuropathy instead of studies reporting about chronic neuropathy more than a year after treatment. Therefore, the influence of oxaliplatin administration on the development of chronic neuropathy remains unclear. A systematic literature review on the influence of oxaliplatin administration (e.g. cumulative dose, dose intensity, number of cycles and combination regimen) on the long-term prevalence of oxaliplatin-induced peripheral neuropathy (O-IPN) at least 12 months after termination of chemotherapy was performed in chapter 5. Fourteen articles ( $n=3,869$ patients) were included and the majority of these studies were of high quality. They reported that O-IPN was still present in a great amount of patients $\geq 12$ months after termination of therapy. Only six studies directly assessed the relationship between oxaliplatin administration and neuropathy. Of these studies, five did find a relation between neuropathy and higher cumulative dose, whereas one study did not find a relation. However, given the heterogeneous definitions and tools utilized in the studies of O-IPN, no firm conclusions could be drawn regarding oxaliplatin administration and the development of long-term O-IPN. Hence, we concluded that a higher cumulative dose is likely to be a predicting factor for the development of long-term O-IPN.

As no firm conclusions could be drawn on the influence of oxaliplatin administration on the development of long-term CIPN, additional information on the actually delivered oxaliplatin treatment for a subgroup of CRC survivors was collected (Chapter 6). In total 207 patients, diagnosed with CRC between 2000 and 2009, underwent adjuvant treatment with oxaliplatin. Those patients were on average 4.0 years since diagnosis, and were diagnosed with colon cancer stage III in $84 \%$. Analysis demonstrated that a higher cumulative dose was associated with the development of long-term CIPN. Dose intensity and time delay did not influence CIPN. Patients with documented acute neuropathy during treatment receiving a dose 
modification (reduction and/or interval prolongation) because of neuropathy ( $n=50$ ) reported significantly worse sensory scores after adjustment for cumulative doses, than those who did not receive a dose reduction because of neuropathy $(n=96)$. In conclusion, monitoring symptoms of neuropathy during treatment is important as the risk of developing persistent CIPN may only be reduced by decreasing the cumulative dose of oxaliplatin, whereas delaying chemotherapy cycles probably is not beneficial. In addition, patients receiving a dose reduction because of acute neuropathy, are still at risk of developing long-term CIPN.

Another group of patients in whom dose limiting neurotoxicity, referred to as CIPN, is a common adverse effect are the more vulnerable patients with MM. In Chapter 7 the prevalence of self-reported chemotherapy-induced peripheral neuropathy (CIPN) symptoms, its association with sociodemographic and clinical characteristics, and the amount of treatment dose modifications and its influence on CIPN in a populationbased cohort of MM patients was evaluated. In total $156 \mathrm{MM}$ patients completed the EORTC QLQ-CIPN20 on average 3.3 years after diagnosis (65\% response). Sociodemographic and clinical characteristics were available from the NCR and the Population-based Haematological Registry for Observational Studies (PHAROS). This study showed that considerable neuropathy was reported by more than half of MM patients, with $53 \%$ percent of patients reporting at least one and on average three neuropathy symptoms that bothered them 'quite a bit' or 'very much' during the past week. Tingling toes or feet were most reported. Multivariate analysis showed that thalidomide, and especially a higher received cumulative dose, was associated with the severity of neuropathy, whereas CIPN was not associated with age, sex, time since last course of therapy, number of prior therapies and comorbidity. Dose was modified in one third of the patients because of CIPN and in spite of this modified dose, patients still reported a trend towards higher neuropathy scores without altered response rates because of the dose modification. Therefore, modifying the dose at a lower level of CIPN as assessed with a more reliable assessment tool seems warranted.

\section{General discussion}

The prevalence of cancer is increasing as a result of growing incidence of cancer due to the aging population and increasing cure rates. Consequently, a paradigm shift has occurred in the field of cancer research. Whereas quantity of life was the primary goal at first, quality of life and patients' well-being are rising in importance as more patients and cancer survivors are confronted with the consequences of cancer and its treatment and, furthermore, prognosis is already improving because of screening 
programs and earlier referral with as a result a smaller absolute gain from adjuvant systemic treatments in some patients. ${ }^{1}$ One of the growing topics in the field of research is the common dose limiting side effect CIPN, especially since no preventive method or treatment is available for this potentially disabling and irreversible adverse event of commonly used chemotherapeutic agents.

\section{The burden of CIPN}

It is assumed that CIPN has a negative influence on patients' $\mathrm{QOL},{ }^{1}$ mainly because it may result in serious limitations in daily activities. ${ }^{2,3}$ For example, symptoms such as tingling, numbness, and aching or burning pain in the fingers or hands may cause problems with regular activities such as buttoning up a blouse, writing with a pen, or opening a jar or bottle. Likewise, comparable symptoms in the toes and feet may cause problems with walking, climbing stairs or driving a car. Although it is assumed that CIPN can have a negative influence on patients daily functioning and therefore QOL, little has been published on the association between CIPN and QOL. ${ }^{1,4}$ Many studies that described CIPN and QOL described them separately, and did not directly assess their relationship. ${ }^{5-18}$ However, ten studies which evaluated the association between CIPN and QOL reported that CIPN negatively influenced patients' QOL, ${ }^{19-27}$ and three other studies did not find an association. ${ }^{28-30}$

Nonetheless, it remains difficult to draw firm conclusions on this matter as the studies which evaluated the association between CIPN and QOL differed tremendously. To begin with, different approaches to assess CIPN were used whereas standardization of CIPN assessment, subjectively by patient-reported outcomes and/or objectively by physical examination or nerve conduction studies is essential for appropriate evaluation and comparison of studies. Further, the studies were also very heterogeneous in the applied study design, time since diagnosis, the included patient population and chemotherapeutic agents used, ${ }^{19-30}$ which makes comparison between studies difficult. In this thesis (Chapter 2, 3, 4), it was observed that CIPN was frequently experienced in patients treated with platinum derivatives and taxanes, in CRC survivors treated with oxalipatin, and in patients with MM treated with immunemodulatory drugs thalidomide and lenalidomide, the proteasome inhibitor bortezomib and/or vincristine, even months to years after last treatment. It was also observed that those who reported the highest and therefore worst CIPN scores reported worse QOL compared with patients who reported less severe CIPN. These and findings from others suggest that severe CIPN is likely to be negatively associated with QOL. However, because of the diversity of the studied patient populations and differences in CIPN and QOL measurements, further research is warranted. In order to do so, the assessment of CIPN and QOL should become more standardized. Preferably 
this assessment is easy to use in daily practice, and incorporate both a subjective patient-reported outcome and an objective measurement. In addition, to define a causal relationship between CIPN and QOL, these studies should preferably be prospective in nature assessing CIPN and $\mathrm{QOL}$ both before, during and after treatment.

Furthermore, besides reporting statistically significant differences in QOL and CIPN, it is also important to determine if these differences are of clinical importance. A statistically significant difference is a difference that is unlikely being caused by chance, and is purely based on mathematical considerations. ${ }^{31}$ However, in health care a significant difference, made by an intervention for example, is not always considered a clinically important difference as the change is not considered meaningful and worthwhile for the patient. ${ }^{31}$ Therefore it is important to determine which difference is, besides being statistically significant, also a clinically important difference for the patient and may lead to alteration in clinical decisions. In this thesis, the differences in QOL between patients with or without severe CIPN were, besides statistically significant, also clinically important to patients determined with Norman's 'rule of thumb', in which a difference of $\approx 0.5$ SD points to a threshold of discriminant change. ${ }^{32}$ The differences were varying from large to small clinical relevance according to the reported guidelines ${ }^{32,33}$ (Chapter 3,4 ). This was observed in both long-term CRC survivors and in the more vulnerable MM patients. Although we reported that those differences were clinically important, it remains difficult to determine if the impact of CIPN is the same in those different patient populations. The long-term CRC survivors may have other expectations of daily functioning and life, including social activities and work, compared with the frequently still actively treated patients with MM with considerably worse prognosis.

\section{The burden of CIPN underestimated by flaws in clinical trials}

QOL is not only determined by CIPN, but also by numerous other chemotherapyinduced toxicities, like fatigue, gastrointestinal and sometimes cardiovascular symptoms. ${ }^{34}$ Two studies reported that fatigue, one of the most frequently observed and often debilitating toxicities of chemotherapy, was associated with other common side effects such as CIPN, and greater levels of fatigue were related with worse symptom severity. ${ }^{35,36}$ In chapter $\mathbf{3}$ and $\mathbf{4}$ of this thesis it was also shown that patients who reported the highest CIPN symptom burden also reported higher levels of fatigue and pain. These observations might indicate that not only certain toxicities are related, but also that they might worsen one another, and all together influencing patients' QOL. Mainly grades three or four adverse events are reported in randomized 
clinical trials (RCTs), but grade one and two adverse events, which are already often underreported by clinicians, ${ }^{37}$ are therefore also undervalued. Especially if the side effects are interrelated. In addition, not only the underreporting by clinicians of these subjective toxicities in clinical trials is of concern, also the scheduling of adverse event assessment during therapy is crucial. Adverse events are most frequently assessed before the preceding therapy, however shortly after chemotherapy toxicity levels are the highest. Accordingly, the burden of the adverse events for patients is probably even more during the treatment period than reported. Therefore, it should be mentioned that the burden of chemotherapy is not only determined by the severity of the adverse events, but also by the timeframe and combination of all adverse events together. Therefore, the burden of chemotherapy should better be expressed in the total number and level (including grade I and II) of experienced toxicities multiplied by the timeframe between cycles they report those side effects. In daily care this might be difficult to define, however an assessment tool to determine this total burden would be valuable.

\section{Difficulties in the assessment of CIPN}

The assessment of CIPN is a major topic of debate. Many assessment tools for measuring CIPN symptoms have been investigated, varying from objective measurements, physician-based grading scales, to subjective patient-reported outcome measurements. $^{38}$ However, there is no consensus on which factors, subjective or objective measurements, are most important in determining clinical severity of neuropathy. ${ }^{38,39}$ Therefore, different measurements are used which makes comparison of CIPN symptom level between studies difficult. ${ }^{38,39}$ In addition, the development of standardized measurements to assess the level and severity of CIPN is required in order to investigate strategies for the prevention and treatment of CIPN in a reliable way.

Furthermore, another issue is that the currently existing measurements are not developed for use in daily clinical practice, although this is highly needed as treatment may be modified based on a reliable tool. ${ }^{38}$ Since the objective CIPN measurements are not patient friendly, time-consuming, expensive, and often not sensitive enough to detect beginning or mild CIPN, questionnaires are preferred. ${ }^{39}$ Those questionnaires provide more insight into the subjective sensory symptoms and their influence on patients' daily functioning. Questionnaires capture issues which are not confirmed by the objective measurements. ${ }^{37,38,40,41}$ However, it lacks objective confirmation and clinicians are reticent to base clinical decision making on patients experience only. Though, a recent study confirmed that clinicians tend to underreport 
toxicities, and therefore the use of patient-reported outcome measurements was highly recommended in the assessment of adverse events. ${ }^{37}$

In addition, distinction between grades of CIPN remains difficult for clinicians. ${ }^{40}$ Nonetheless, guidelines necessary to decide on dose modifications of chemotherapy in daily clinical practice are mainly based on the physician-based National Cancer Institute-Common Toxicity Criteria ( $\mathrm{NCl}-\mathrm{CTC}$ ). Therefore, CIPN questionnaires, although recommended, are difficult to use for this purpose in daily clinical practice. ${ }^{42}$ Dose modifications should only be applied with caution as they can influence treatment outcome. Therefore, it is of utmost importance that a simple, patientfriendly and reliable assessment tool, including patient-reported information, for daily clinical practice will be developed. One of this attempts are the patient-reported outcome (PRO)-CTCAE criteria in which patient-reported measurements are integrated in NCl-CTCAE criteria. ${ }^{43,44}$ In chapter $\mathbf{4}$ of this thesis, we present the selfreported Indication Common Toxicity Criteria-grading Peripheral Neuropathy Questionnaire (ICPNQ) as a valid instrument to distinguish the highest CIPN CTCgrades from the lower CTC-grades, which may be valuable for the decision to modify the dose of chemotherapy in patients with MM. Hence, this questionnaire provides in the need to give a standardized indication regarding the CTC-grades of CIPN, while it overcomes the concern of underreporting CIPN by clinicians. Therefore this is a valuable tool which can be used in daily clinical practice. To evaluate the ability to monitor the development of CIPN over time, and to compare the CTC-grades derived from this self-reported questionnaire with physician reported CTC-grades, further prospective evaluation of this instrument is needed.

\section{Towards personalized medicine, identifying patients at risk of developing CIPN}

At the moment, alternative dosing regimens and treatment modification schemes are the only way to prevent moderate CIPN to become more severe. Therefore, identifying patients at risk of developing CIPN is of major importance. In chapter $\mathbf{5}$ and 6 of this thesis it was shown that a higher cumulative dose of oxaliplatin was associated with the development of long-term CIPN in CRC survivors. Therefore, monitoring symptoms of neuropathy during treatment is important as the risk of developing persistent CIPN may only be reduced by decreasing the cumulative dose of oxaliplatin on time. Nevertheless, not all patients receiving a certain cumulative dose of a neurotoxic agent develop CIPN. Additionally, the amount of symptoms and reversibility of CIPN varies strongly between patients. This could be explained by the findings of recent studies demonstrating that certain patients, who are treated with oxaliplatin, might be more at risk of developing CIPN due to variants in encoding 
genes of ion channels in the central nervous system. ${ }^{45-49}$ In addition, a Dutch study also suggested an interaction between myeloma-related factors and the genetic background of patients with $\mathrm{MM}$ in the development of CIPN, with different molecular pathways being implicated in bortezomib-induced and vincristine-induced peripheral neuropathy. ${ }^{50}$

As the area of genomics studies is still in its infancy and not routinely used in daily clinical practice, it is important to take into account other patient characteristics when deciding on the best treatment for a particular patient with the least toxicity. For instance, pre-existing neuropathy, age, alcohol abuse, and comorbidity such as diabetes mellitus are factors related to the development or aggravation of CIPN, ${ }^{51,52}$ and the presence of those risk factors may therefore be a reason to restrain certain chemotherapies for some patients. Therefore, preferably for the start of chemotherapy patients should be screened for all those factors. Furthermore, an American trial showed that patients aged $\geq 70$ with stage III colon cancer may not benefit from adding oxaliplatin to oral fluoropyrimidines in the adjuvant setting, and therefore CIPN may also be prevented. ${ }^{53}$ Also the stage and predicted response rate of disease should be reckoned when choosing the best treatment. As mentioned above, the aim of medical treatment is not only prolongation of life, but also the preservation of its quality. For example, in chapter $\mathbf{7}$ more than half of patients with MM were confronted with considerable neuropathy due to neurotoxic treatment and in spite of dose modification patients reported a trend towards higher levels of neuropathy. Due to improving overall survival patients are therefore confronted with this side effect for a longer period. Nevertheless, the curation rate of MM remains scarce.

Given that there is still no proven therapy for CIPN and due to the burden of CIPN on the daily lives of patients, identifying the clinical characteristics and genetic variations in patients who are prone to develop CIPN is of major importance. In that way, patients may benefit from personalized medicine as patients and clinicians together can decide to decrease the cumulative chemotherapy dose on time or restrain certain types of chemotherapy with the goal to prevent or minimize CIPN. This shared decision making should be taken under the condition that the patients have received proper information about the different treatment options and about CIPN and the consequences of this potentially severe side effect. 


\section{Prevention and treatment of CIPN}

Recent practical guidelines could give no strong recommendations regarding the pharmacological prevention or treatment of CIPN. ${ }^{39,54,55}$ Only a weak recommendation for duloxetine, gabapentin, or pregabalin for chemotherapy-induced neuropathic pain could be given. This is mainly because trials tend to be small and heterogeneous in patient population, the applied neurotoxic agent, and time of CIPN assessment. ${ }^{39,54}$ In addition, the absence of a consensus in outcome measurements of CIPN, as earlier mentioned, makes comparison of studies and drawing firm conclusions on their results difficult. Consequently, recommendations on the management of neuropathic pain in cancer patients are usually based on studies concerning painful diabetic neuropathy, post-herpetic neuralgia and trigeminal neuralgia which are 'benign' neuropathic pain conditions. ${ }^{56}$ However, CIPN is different from those forms of neuropathic pain with respect to pathophysiology and symptomatology, and therefore the development of a better suitable treatment is highly needed. ${ }^{39}$

Non-pharmacologic interventions are not considered in the recent guideline from the American Society of Oncology. ${ }^{39}$ They state that a number of non-pharmacologic interventions have been investigated, but due to the paucity of RCTs the inclusion of those studies in the systematic review have been impeded. ${ }^{39}$ However, given the lack of evidence for pharmacological treatment, non-randomized trials could also provide valuable information, and give a direction to future research. ${ }^{57}$ For example, a Danish cohort study showed less CIPN in docetaxel treated patients who wore frozen gloves or socks. ${ }^{58}$ Following this study, we hypothesized that wearing frozen gloves during chemotherapy could be effective in preventing CIPN in the hands. Frozen gloves may induce vasoconstriction and decrease uptake of chemotherapy due to less biochemical activity within the neuronal cells, and therefore cause less axonal damage. Therefore, we conducted a pilot study in 30 cancer patients that showed a trend with less neuropathic pain and discomfort in a frozen glove protected hand compared with a control hand. ${ }^{59}$ We expended this pilot study to a multicenter, randomized controlled study to evaluate the efficacy of frozen gloves in the prevention of CIPN due to treatment with neurotoxic agents. Other investigated nonpharmacological ways of preventing or treating CIPN are the use of dietary supplements, scrambler therapy or rehabilitation programs. The most extensive investigated dietary supplements are the natural herbal Goshajinkigan (GJG) and antioxidant acetyl-L-carnitine (ALC). ${ }^{60-66}$ GJG showed to cause less grade III CIPN in patients using GJG during chemotherapy in four Japanese studies ${ }^{60-62}$ and results on ALC were encouraging in two studies, but less conclusive in others. ${ }^{63-66}$ Nonetheless, a previous study reported that serum ALC concentrations were significantly lower in cachectic patients when compared to healthy volunteers. ${ }^{67}$ Furthermore, fatigue among patients with cancer and with low serum-carnitine levels has reported to 
become less after treatment with ALC. ${ }^{68}$ Therefore, we suggest that this simple dietary supplement has to be investigated more thoroughly in which precise metabolic and pharmacokinetic analyses are required. Scrambler therapy, in which noninvasive cutaneous electrostimulation is used to treat neuropathic pain, showed a short-term reduction in pain in patients with CIPN in two small studies. ${ }^{69,70}$ Those studies about the use of dietary supplements and scrambler therapy report encouraging results, nevertheless all those studies suffer from small sample sizes and heterogeneity in CIPN assessment, and therefore are not recommended at the moment.

Nowadays in the Netherlands, rehabilitation programs however are offered to cancer patients in standard cancer care, as they have demonstrated physiological and psychological benefits. ${ }^{71,72}$ Nonetheless, most of the studies investigating rehabilitation programs were indeed also not RCTs, used small sample sizes and did not consider the special needs of patients with $\mathrm{CIPN}^{71,73}$ Only one small pilot study reported improved balance due to intensive rehabilitation in patients with CIPN. ${ }^{72}$ Moreover, no trials investigated the effect of rehabilitation on coordination and muscle strength in cancer patients with CIPN. A Dutch systematic review, investigating the effect of whole body vibration on coordination and muscle strength, was not able to include patients with peripheral neuropathy due to chemotherapy. ${ }^{73}$ Rehabilitation programmes performed in alternative populations, such as elderly or patients with diabetic peripheral neuropathy, have shown to be effective in diminishing the development of neuropathy. ${ }^{74}$ In a recent study we reported that meeting the physical activity guideline is associated with less severe neuropathy in CRC survivors and therefore physical activity interventions are an interesting subject for future research in treating and preventing CIPN. ${ }^{75}$ Therefore, studies investigating nonpharmacological interventions such as dietary supplements or rehabilitation modalities in patients with CIPN should be encouraged. Besides, those interventions can easily be incorporated in daily clinical care, and consequently are of particular interest.

In conclusion, prevention and treatment of CIPN remains unproven. Therefore, more studies investigating both pharmacological and non-pharmacological interventions to prevent or treat CIPN are required. Meanwhile, alternative dosing regimens, early detection and treatment modification schemes are necessary to limit CIPN. In addition, patients should be informed properly on the implications of this side effect which is illustrated by the quote of the 50 -year old CRC patient in the introduction. 


\section{Future perspectives}

In this current thesis it is shown that CIPN is a common dose limiting and potentially severe side effect of frequently used chemotherapeutic agents, which can be experienced until years after treatment with a negative influence on patients' QOL. With the increasing number of patients confronted with this side effect, and the fact that there is no proven therapy for CIPN, it is likely that more patients will be confronted with CIPN. Much is still unknown about this potentially disabling adverse event and the studies included in this thesis are just the first steps of a long journey in which still many facets need to be discovered. In Table 8.1 a summary is given of the current knowledge of CIPN, results of this thesis and the future perspectives. In summary, more research to explore CIPN is necessary.

To begin with, it is of major importance that we understand the underlying mechanisms of CIPN necessary to define potential targets for the prevention and treatment of CIPN, especially since an increasing amount of patients is confronted with the short- and long-term consequences of CIPN.

Furthermore, in order to investigate strategies for the prevention and treatment of CIPN it is vital to be able to assess the severity of CIPN in a reliable way. Future studies should therefore also focus on the development of a valid and reliable measurement to assess CIPN. This tool should, besides being a valid and reliable tool, be patientfriendly, responsive to change in CIPN, and easy to use in daily clinical practice. In addition, it should incorporate at least a patient-reported outcome measurement.

Moreover, studies investigating the influence of CIPN on patients' QOL and the burden of associated and lower grade adverse events are stimulated. Those studies should preferably determine not only statistically significant differences, but also report if these differences are clinically important to patients.

In addition, with personalized medicine becoming more integrated in future cancer care, it is important to identify risk factors for the development of CIPN. Therefore studies investigating the influence of sociodemographic and chemotherapy administration on the development of CIPN, and studies investigating genetic variations making patients more prone to develop CIPN are encouraged. Consequently in the future the best treatment for each individual patient with the least toxicity can be chosen after close consideration between patient and clinician. 


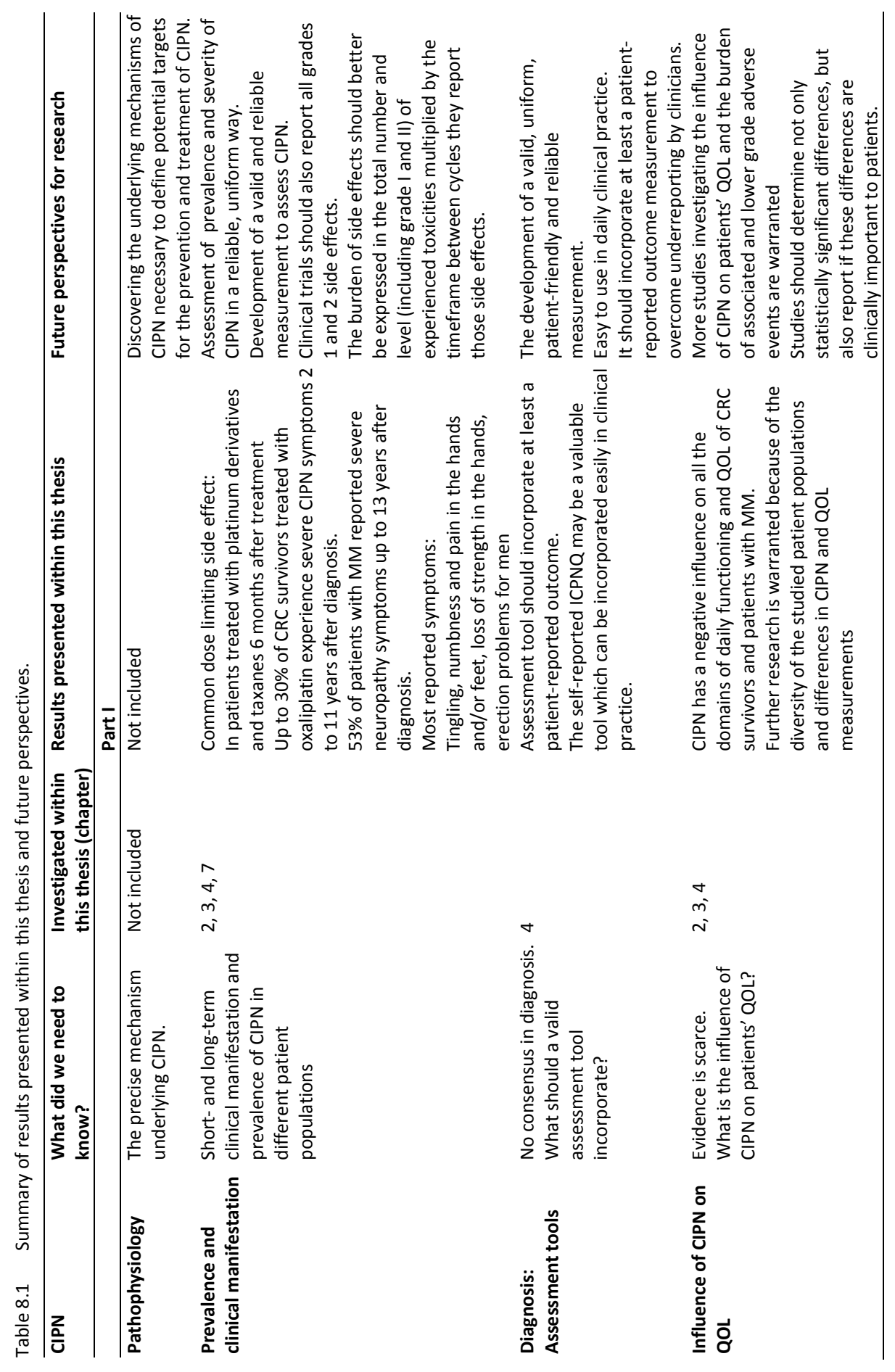




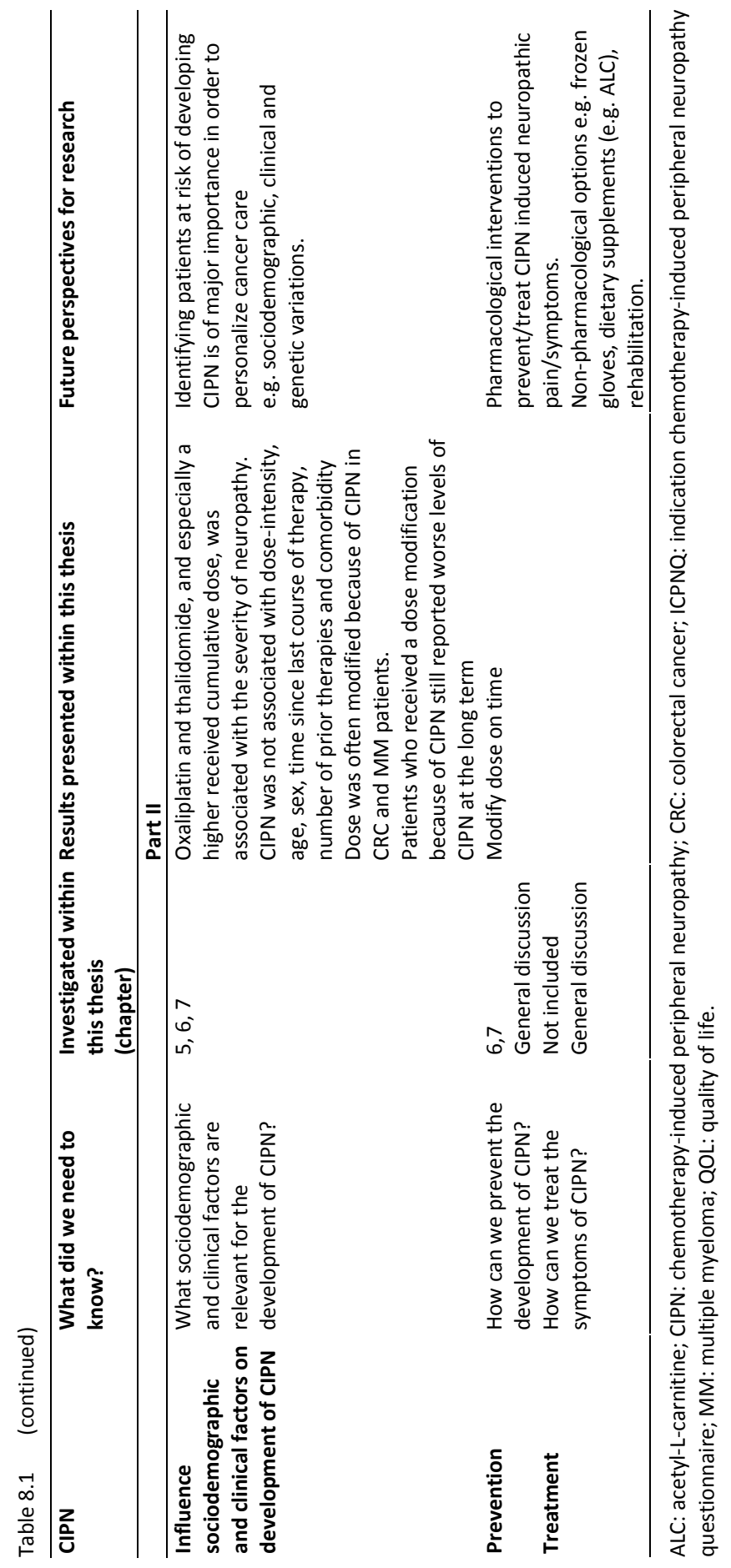




\section{References}

1. Mols F, Beijers T, Vreugdenhil G, van de Poll-Franse L. Chemotherapy-induced peripheral neuropathy and its association with quality of life: a systematic review. Support Care Cancer. 2014;22:2261-2269.

2. Tofthagen C. Surviving chemotherapy for colon cancer and living with the consequences. J Palliat Med. 2010;13(11):1389-1391.

3. Bakitas MA. Background noise: the experience of chemotherapy-induced peripheral neuropathy. Nurs Res. 2007;56(5):323-331.

4. Scheel A, Beijers AJ, Mols F, Faber CG, Vreugdenhil G. [Chemotherapy-induced peripheral neuropathy; impact on quality of life]. Ned Tijdschr Geneeskd. 2014;158:A7455.

5. Bezjak A, Tu D, Bacon M, Osoba D, Zee B, Stuart G, et al. Quality of life in ovarian cancer patients: comparison of paclitaxel plus cisplatin, with cyclophosphamide plus cisplatin in a randomized study. J Clin Oncol. 2004;22(22):4595-4603.

6. Butler L, Bacon M, Carey M, Zee B, Tu D, Bezjak A. Determining the relationship between toxicity and quality of life in an ovarian cancer chemotherapy clinical trial. J Clin Oncol. 2004;22(12):2461-2468.

7. Brundage M, Gropp M, Mefti F, Mann K, Lund B, Gebski V, et al. Health-related quality of life in recurrent platinum-sensitive ovarian cancer--results from the CALYPSO trial. Ann Oncol. 2012;23(8):2020-2027.

8. Bruner DW, Barsevick A, Tian C, Randall M, Mannel R, Cohn DE, et al. Randomized trial results of quality of life comparing whole abdominal irradiation and combination chemotherapy in advanced endometrial carcinoma: A gynecologic oncology group study. Qual Life Res. 2007;16(1):89-100.

9. Cella D, Huang HQ, Monk BJ, Wenzel L, Benda J, McMeekin DS, et al. Health-related quality of life outcomes associated with four cisplatin-based doublet chemotherapy regimens for stage IVB recurrent or persistent cervical cancer: a Gynecologic Oncology Group study. Gynecol Oncol. 2010;119(3):531-537.

10. Hershman DL, Weimer LH, Wang A, Kranwinkel G, Brafman L, Fuentes D, et al. Association between patient reported outcomes and quantitative sensory tests for measuring long-term neurotoxicity in breast cancer survivors treated with adjuvant paclitaxel chemotherapy. Breast Cancer Res Treat. 2011;125(3):767-774.

11. Shimozuma K, Ohashi Y, Takeuchi A, Aranishi T, Morita S, Kuroi K, et al. Taxane-induced peripheral neuropathy and health-related quality of life in postoperative breast cancer patients undergoing adjuvant chemotherapy: N-SAS BC 02, a randomized clinical trial. Support Care Cancer. 2012;20(12):3355-3364.

12. Tofthagen C. Patient perceptions associated with chemotherapy-induced peripheral neuropathy. Clin J Oncol Nurs. 2010;14(3):E22-28.

13. Hilpert F, Stahle A, Tome O, Burges A, Rossner D, Spathe K, et al. Neuroprotection with amifostine in the first-line treatment of advanced ovarian cancer with carboplatin/paclitaxel-based chemotherapy-a double-blind, placebo-controlled, randomized phase II study from the Arbeitsgemeinschaft Gynakologische Onkologoie (AGO) Ovarian Cancer Study Group. Support Care Cancer. 2005;13(10): 797-805.

14. Kautio AL, Haanpaa M, Saarto T, Kalso E. Amitriptyline in the treatment of chemotherapy-induced neuropathic symptoms. J Pain Symptom Manage. 2008;35(1):31-39.

15. Lee SM, Rudd R, Woll PJ, Ottensmeier C, Gilligan D, Price A, et al. Randomized double-blind placebocontrolled trial of thalidomide in combination with gemcitabine and Carboplatin in advanced nonsmall-cell lung cancer. J Clin Oncol. 2009;27(31):5248-5254.

16. Richter R, Oskay-Oezcelik G, Chekerov R, Pilger A, Hindenburg HJ, Sommer H, et al. Health-related quality of life during sequential chemotherapy with carboplatin followed by weekly paclitaxel in advanced ovarian cancer: a multicenter phase ii study of the North Eastern German Society of Gynecological Oncology. Anticancer Res. 2012;32(9):3969-3976.

17. Smith EM, Pang H, Cirrincione C, Fleishman S, Paskett ED, Ahles T, et al. Effect of duloxetine on pain, function, and quality of life among patients with chemotherapy-induced painful peripheral neuropathy: a randomized clinical trial. JAMA. 2013;309(13):1359-1367. 
18. Wenzel LB, Huang HQ, Armstrong DK, Walker JL, Cella D, Gynecologic Oncology G. Health-related quality of life during and after intraperitoneal versus intravenous chemotherapy for optimally debulked ovarian cancer: a Gynecologic Oncology Group Study. J Clin Oncol. 2007;25(4):437-443.

19. Baz R, Lin HM, Hui AM, Harvey RD, Colson K, Gallop K, et al. Development of a conceptual model to illustrate the impact of multiple myeloma and its treatment on health-related quality of life. Support Care Cancer. 2015;23(9):2789-2797.

20. Jones D, Zhao F, Brell J, Lewis MA, Loprinzi CL, Weiss M, et al. Neuropathic symptoms, quality of life, and clinician perception of patient care in medical oncology outpatients with colorectal, breast, lung, and prostate cancer. J Cancer Surviv. 2015;9(1):1-10.

21. Cella D, Peterman A, Hudgens S, Webster K, Socinski MA. Measuring the side effects of taxane therapy in oncology: the functional assesment of cancer therapy-taxane (FACT-taxane). Cancer. 2003;98(4): 822-831.

22. Driessen $\mathrm{CM}$, de Kleine-Bolt KM, Vingerhoets AJ, Mols F, Vreugdenhil G. Assessing the impact of chemotherapy-induced peripheral neurotoxicity on the quality of life of cancer patients: the introduction of a new measure. Support Care Cancer. 2012;20(4):877-881.

23. Griffith KA, Couture DJ, Zhu S, Pandya N, Johantgen ME, Cavaletti G, et al. Evaluation of chemotherapy-induced peripheral neuropathy using current perception threshold and clinical evaluations. Support Care Cancer. 2014;22(5):1161-1169.

24. Kim BJ, Park HR, Roh HJ, Jeong DS, Kim BS, Park KW, et al. Chemotherapy-related polyneuropathy may deteriorate quality of life in patients with B-cell lymphoma. Qual Life Res. 2010;19(8):1097-1103.

25. Ostchega Y, Donohue M, Fox N. High-dose cisplatin-related peripheral neuropathy. Cancer Nurs. 1988;11(1):23-32.

26. Sorbe B, Graflund M, Horvath G, Swahn M, Boman K, Bangshoj R, et al. Phase II study of docetaxel weekly in combination with carboplatin every 3 weeks as first-line chemotherapy in stage IIB to stage IV epithelial ovarian cancer. Int J Gynecol Cancer. 2012;22(1):47-53.

27. Mols F, Beijers T, Lemmens V, van den Hurk CJ, Vreugdenhil G, van de Poll-Franse LV. Chemotherapyinduced neuropathy and its association with quality of life among 2- to 11-year colorectal cancer survivors: results from the population-based PROFILES registry. J Clin Oncol. 2013;31(21):2699-2707.

28. Calhoun EA, Welshman EE, Chang CH, Lurain JR, Fishman DA, Hunt TL, et al. Psychometric evaluation of the Functional Assessment of Cancer Therapy/Gynecologic Oncology Group-Neurotoxicity (Fact/GOG-Ntx) questionnaire for patients receiving systemic chemotherapy. Int J Gynecol Cancer. 2003;13(6):741-748.

29. Morita S, Kobayashi K, Eguchi K, Matsumoto T, Shibuya M, Yamaji Y, et al. Influence of clinical parameters on quality of life during chemotherapy in patients with advanced non-small cell lung cancer: application of a general linear model. Jpn J Clin Oncol. 2003;33(9):470-476.

30. Ramchandren S, Leonard M, Mody RJ, Donohue JE, Moyer J, Hutchinson R, et al. Peripheral neuropathy in survivors of childhood acute lymphoblastic leukemia. J Peripher Nerv Syst. 2009;14(3):184-189.

31. Copay AG, Subach BR, Glassman SD, Polly DW, Jr., Schuler TC. Understanding the minimum clinically important difference: a review of concepts and methods. Spine J. 2007;7(5):541-546.

32. Norman GR, Sloan JA, Wyrwich KW. Interpretation of changes in health-related quality of life: the remarkable universality of half a standard deviation. Med Care. 2003;41(5):582-592.

33. Cocks K, King MT, Velikova G, Martyn St-James M, Fayers PM, Brown JM. Evidence-based guidelines for determination of sample size and interpretation of the European Organisation for the Research and Treatment of Cancer Quality of Life Questionnaire Core 30. J Clin Oncol. 2011;29(1):89-96.

34. Beijers AJ, Mols F, van den Hurk CJ, Vreugdenhil A. Are chemotherapy-associated symptoms underestimated? A view beyond common toxicity criteria. Acta Oncol. 2016;55(4):516-518.

35. Efficace F, Gaidano G, Breccia M, Criscuolo M, Cottone F, Caocci G, et al. Prevalence, severity and correlates of fatigue in newly diagnosed patients with myelodysplastic syndromes. $\mathrm{Br} J$ Haematol. 2015;168(3):361-370.

36. Savard J, Ivers H, Savard MH, Morin CM. Cancer treatments and their side effects are associated with aggravation of insomnia: Results of a longitudinal study. Cancer. 2015;121(10):1703-1711. 
37. Di Maio M, Gallo C, Leighl NB, Piccirillo MC, Daniele G, Nuzzo F, et al. Symptomatic toxicities experienced during anticancer treatment: Agreement between patient and physician reporting in three randomized trials. J Clin Oncol. 2015;33(8):910-915.

38. Cavaletti G, Frigeni B, Lanzani F, Mattavelli L, Susani E, Alberti P, et al. Chemotherapy-Induced Peripheral Neurotoxicity assessment: a critical revision of the currently available tools. Eur J Cancer. 2010;46(3):479-494.

39. Hershman DL, Lacchetti C, Dworkin RH, Lavoie Smith EM, Bleeker J, Cavaletti G, et al. Prevention and Management of Chemotherapy-Induced Peripheral Neuropathy in Survivors of Adult Cancers: American Society of Clinical Oncology Clinical Practice Guideline. J Clin Oncol. 2014;32(18):1941-1967.

40. Postma TJ, Heimans JJ, Muller MJ, Ossenkoppele GJ, Vermorken JB, Aaronson NK. Pitfalls in grading severity of chemotherapy-induced peripheral neuropathy. Ann Oncol. 1998;9(7):739-744.

41. Basch E, lasonos A, McDonough T, Barz A, Culkin A, Kris MG, et al. Patient versus clinician symptom reporting using the National Cancer Institute Common Terminology Criteria for Adverse Events: results of a questionnaire-based study. Lancet Oncol. 2006;7(11):903-909.

42. Zweegman S, Lokhorst HM, Levin MD, Waal de E, Bos GMJ, Kersten MJ, et al. Guidelines for the treatment of multiple myeloma 2012. The Dutch Journal of Hematology. 2012;8:300-320.

43. Basch E, Reeve BB, Mitchell SA, Clauser SB, Minasian LM, Dueck AC, et al. Development of the National Cancer Institute's patient-reported outcomes version of the common terminology criteria for adverse events (PRO-CTCAE). J Natl Cancer Inst. 2014;106(9).

44. Dueck AC, Mendoza TR, Mitchell SA, Reeve BB, Castro KM, Rogak LJ, et al. Validity and Reliability of the US National Cancer Institute's Patient-Reported Outcomes Version of the Common Terminology Criteria for Adverse Events (PRO-CTCAE). JAMA Oncol. 2015;1(8):1051-1059.

45. Argyriou AA, Antonacopoulou AG, Scopa CD, Kottorou A, Kominea A, Peroukides S, et al. Liability of the voltage-gated sodium channel gene SCN2A R19K polymorphism to oxaliplatin-induced peripheral neuropathy. Oncology. 2009;77(3-4):254-256.

46. Argyriou AA, Cavaletti G, Antonacopoulou A, Genazzani AA, Briani C, Bruna J, et al. Voltage-gated sodium channel polymorphisms play a pivotal role in the development of oxaliplatin-induced peripheral neurotoxicity: Results from a prospective multicenter study. Cancer. 2013;119:3570-3577.

47. Faber CG, Hoeijmakers JG, Ahn HS, Cheng X, Han C, Choi JS, et al. Gain of function Nanu1.7 mutations in idiopathic small fiber neuropathy. Ann Neurol. 2012;71(1):26-39.

48. Faber CG, Lauria G, Merkies IS, Cheng X, Han C, Ahn HS, et al. Gain-of-function Nav1.8 mutations in painful neuropathy. Proc Natl Acad Sci U S A. 2012;109(47):19444-19449.

49. Huang J, Han C, Estacion M, Vasylyev D, Hoeijmakers JG, Gerrits MM, et al. Gain-of-function mutations in sodium channel NaV1.9 in painful neuropathy. Brain. 2014;137(Pt 6):1627-1642.

50. Broyl A, Corthals SL, Jongen JL, van der Holt B, Kuiper R, de Knegt $Y$, et al. Mechanisms of peripheral neuropathy associated with bortezomib and vincristine in patients with newly diagnosed multiple myeloma: a prospective analysis of data from the HOVON-65/GMMG-HD4 trial. Lancet Oncol. 2010;11(11):1057-1065.

51. Vincenzi B, Frezza AM, Schiavon G, Spoto C, Silvestris N, Addeo R, et al. Identification of clinical predictive factors of oxaliplatin-induced chronic peripheral neuropathy in colorectal cancer patients treated with adjuvant Folfox IV. Support Care Cancer. 2013;21(5):1313-1319.

52. Velasco R, Bruna J, Briani C, Argyriou AA, Cavaletti G, Alberti P, et al. Early predictors of oxaliplatininduced cumulative neuropathy in colorectal cancer patients. J Neurol Neurosurg Psychiatry. 2014;85(4):392-398.

53. McCleary NJ, Meyerhardt JA, Green E, Yothers G, de Gramont A, Van Cutsem E, et al. Impact of age on the efficacy of newer adjuvant therapies in patients with stage II/II colon cancer: findings from the ACCENT database. J Clin Oncol. 2013;31(20):2600-2606.

54. van den Beuken-van Everdingen MH, de Graeff A, Jongen JL, Dijkstra D, Mostovaya I, Vissers KC, et al. Pharmacological treatment of pain in cancer patients: The role of adjuvant analgesics, a systematic review. Pain Pract. 2016.

55. Beijers AJ, Jongen JL, Vreugdenhil G. Chemotherapy-induced neurotoxicity: the value of neuroprotective strategies. Neth J Med. 2012;70(1):18-25. 
56. Finnerup NB, Sindrup SH, Jensen TS. The evidence for pharmacological treatment of neuropathic pain. Pain. 2010;150(3):573-581.

57. Beijers AJ, Verhulst AL, Mols F, Vreugdenhil G. Comment on: 'Subgroup effects in a randomized trial of different types and doses of exercise during breast cancer chemotherapy'. $\mathrm{Br} \mathrm{J}$ Cancer. 2015;112(11):1835.

58. Eckhoff L, Knoop AS, Jensen MB, Ejlertsen B, Ewertz M. Risk of docetaxel-induced peripheral neuropathy among 1,725 Danish patients with early stage breast cancer. Breast Cancer Res Treat. 2013;142(1):109-118.

59. Ophorst J, Beijers T, Derksen L, Houterman S, Vreugdenhil G. Handkoeling ter preventie van chemotherapie geïnduceerde perifere neuropathie: een pilotstudie en overzicht van neuroprotectieve middelen. Oncologica. 2012;2.

60. Kono T, Hata T, Morita S, Munemoto Y, Matsui T, Kojima H, et al. Goshajinkigan oxaliplatin neurotoxicity evaluation (GONE): a phase 2, multicenter, randomized, doubleblind, placebocontrolled trial of goshajinkigan to prevent oxaliplatininduced neuropathy. Cancer Chemother Pharmacol. 2013;72(6):1283-1290.

61. Nishioka M, Shimada M, Kurita N, Iwata T, Morimoto S, Yoshikawa K, et al. The Kampo medicine, Goshajinkigan, prevents neuropathy in patients treated by FOLFOX regimen. Int J Clin Oncol. 2011;16(4):322-327.

62. Kono T, Mamiya N, Chisato N, Ebisawa Y, Yamazaki H, Watari J, et al. Efficacy of goshajinkigan for peripheral neurotoxicity of oxaliplatin in patients with advanced or recurrent colorectal cancer. Evid Based Complement Alternat Med. 2011;2011:418481.

63. Bianchi G, Vitali G, Caraceni A, Ravaglia S, Capri G, Cundari S, et al. Symptomatic and neurophysiological responses of paclitaxel- or cisplatin-induced neuropathy to oral acetyl-L-carnitine. Eur J Cancer. 2005;41(12):1746-1750.

64. Maestri A, De Pasquale Ceratti A, Cundari S, Zanna C, Cortesi E, Crino L. A pilot study on the effect of acetyl-L-carnitine in paclitaxel- and cisplatin-induced peripheral neuropathy. Tumori. 2005;91(2): 135-138.

65. Hershman DL, Unger JM, Crew KD, Minasian LM, Awad D, Moinpour CM, et al. Randomized doubleblind placebo-controlled trial of acetyl-L-carnitine for the prevention of taxane-induced neuropathy in women undergoing adjuvant breast cancer therapy. J Clin Oncol. 2013;31(20):2627-2633.

66. Callander N, Markovina S, Eickhoff J, Hutson P, Campbell T, Hematti P, et al. Acetyl-L-carnitine (ALCAR) for the prevention of chemotherapy-induced peripheral neuropathy in patients with relapsed or refractory multiple myeloma treated with bortezomib, doxorubicin and low-dose dexamethasone: a study from the Wisconsin Oncology Network. Cancer Chemother Pharmacol. 2014;74(4):875-882.

67. Malaguarnera M, Risino C, Gargante MP, Oreste G, Barone G, Tomasello AV, et al. Decrease of serum carnitine levels in patients with or without gastrointestinal cancer cachexia. World J Gastroenterol. 2006;12(28):4541-4545.

68. Graziano F, Bisonni R, Catalano V, Silva R, Rovidati S, Mencarini E, et al. Potential role of levocarnitine supplementation for the treatment of chemotherapy-induced fatigue in non-anaemic cancer patients. Br J Cancer. 2002;86(12):1854-1857.

69. Pachman DR, Weisbrod BL, Seisler DK, Barton DL, Fee-Schroeder KC, Smith TJ, et al. Pilot evaluation of Scrambler therapy for the treatment of chemotherapy-induced peripheral neuropathy. Support Care Cancer. 2015;23(4):943-951.

70. Coyne PJ, Wan W, Dodson P, Swainey C, Smith TJ. A trial of Scrambler therapy in the treatment of cancer pain syndromes and chronic chemotherapy-induced peripheral neuropathy. J Pain Palliat Care Pharmacother. 2013;27(4):359-364.

71. Galvao DA, Newton RU. Review of exercise intervention studies in cancer patients. J Clin Oncol. 2005;23(4):899-909.

72. Cammisuli S, Cavazzi E, Baldissarro E, Leandri M. Rehabilitation of balance disturbances due to chemotherapy induced peripheral neuropathy: a pilot study. Eur J Phys Rehabil Med. 2016;52(4): 479-488. 
73. Verhulst AL, Savelberg HH, Vreugdenhil G, Mischi M, Schep G. Whole-Body Vibration as a Modality for the rehabilitation of peripheral neuropathies: Implications for cancer survivors suffering from chemotherapy-induced peripheral neuropathy. Oncol Rev. 2015;9(1):263.

74. Balducci S, lacobellis G, Parisi L, Di Biase N, Calandriello E, Leonetti F, et al. Exercise training can modify the natural history of diabetic peripheral neuropathy. J Diabetes Complications. 2006;20(4):216-223.

75. Mols F, Beijers AJ, Vreugdenhil G, Verhulst A, Schep G, Husson O. Chemotherapy-induced peripheral neuropathy, physical activity and health-related quality of life among colorectal cancer survivors from the PROFILES registry. J Cancer Surviv. 2015;9(3):512-522. 
Samenvatting

(Dutch summary) 


\section{Samenvatting}

In Nederland worden jaarlijks ongeveer 100.000 patiënten geconfronteerd met de diagnose kanker. Naast een operatie en bestraling is behandeling met medicijnen in de vorm van chemotherapie, een belangrijke hoeksteen in de therapie van vele vormen van kanker. De behandeling met chemotherapie wordt vaak vergezeld door vele bijwerkingen zoals misselijkheid, tekort aan bloedplaatjes en/of witte bloedcellen, en zenuwschade, ook wel chemotherapie-geïnduceerde perifere neuropathie (CIPN) genoemd. Deze zenuwschade, in het vervolg CIPN genoemd, is een veel voorkomende, potentieel ernstige en soms onomkeerbare bijwerking van bepaalde chemotherapieën die gebruikt worden in de behandeling van o.a. darm-, borst-, prostaat-, eierstok- of een vorm van witte bloedcelkanker. CIPN presenteert zich met verschillende klachten zoals tintelingen, gevoelloosheid, brandende pijn en/of een onaangenaam gevoel bij aanraking in de extremiteiten. De klachten beginnen meestal in de tenen en vingers, maar kunnen zich verder uitbreiden naar de gehele armen en benen. Bovendien, kan spierzwakte aan de armen en benen en autonome disfunctie, zoals erectiestoornissen of hartkloppingen, optreden. Deze klachten kunnen zo ernstig zijn dat ze leiden tot problemen met dagelijkse activiteiten zoals het dichtknopen van een blouse, vasthouden van een pen, of het openen van een pot of fles. Ook kunnen problemen met wandelen, traplopen of autorijden ontstaan. Soms is de uitwerking van deze bijwerking zo invaliderend dat het zelfs een reden is om helemaal te stoppen met de behandeling, waardoor mogelijk de effectiviteit van de behandeling in het geding komt.

Er komt steeds meer aandacht voor kwaliteit van leven binnen de oncologie, zowel bij patiënten waarbij kanker in een vroeg stadium van de ziekte wordt ontdekt als bij patiënten die ongeneeslijk ziek zijn verklaard. Het doel van de medische behandeling is niet alleen verlenging van het leven, maar ook het behoud van de kwaliteit ervan. Omdat steeds meer patiënten de diagnose kanker krijgen, het gebruik van chemotherapie toeneemt en de overleving van kanker verbetert, worden steeds meer patiënten geconfronteerd met de korte en lange termijn gevolgen van deze schadelijke bijwerking. Er is echter nog weinig bekend over de invloed van CIPN op de kwaliteit van leven van patiënten. Bovendien is er ondanks meerdere onderzoeken nog geen goede behandeling van de symptomen. Het is daarom van groot belang dat er meer inzicht komt in de symptomen en ernst van deze bijwerking en de invloed ervan op de kwaliteit van leven van patiënten. Bovendien is het van belang om te onderzoeken wat de invloed van chemotherapie toediening is op het ontstaan van deze klachten, zodat patiënten die kans maken op het ontwikkelen van blijvende klachten geïdentificeerd kunnen worden. Dit proefschrift is opgedeeld in twee delen. Deel één beschrijft het optreden en de ernst van de symptomen van CIPN, en de invloed van deze symptomen op de kwaliteit van leven bij patiënten met kanker, op verschillende tijdstippen na de behandeling. Deel twee beschrijft de invloed van 
toediening van chemotherapie op de ontwikkeling van permanente CIPN bij patiënten met dikke darmkanker en witte bloedcel kanker.

\section{Belangrijkste bevindingen van dit proefschrift}

\section{Deel 1- CIPN en de invloed op kwaliteit van leven}

Het doel van hoofdstuk 2 was het beschrijven van het optreden en de ernst van de symptomen van CIPN, veroorzaakt door veel gebruikte chemotherapieën in de behandeling van o.a. dikke darmkanker, borst- en eierstokkanker, 6 maanden na het beëindiging van deze behandeling. Bovendien werden de gevolgen van deze symptomen op het dagelijkse functioneren en de kwaliteit van leven onderzocht. In totaal hebben 43 patiënten met kanker van het Máxima Medisch Centrum vragenlijsten ingevuld over CIPN en kwaliteit van leven. De meerderheid van de patiënten rapporteerden CIPN symptomen in de bovenste (79\%) en onderste extremiteiten (90\%). De meest voorkomende klachten waren gevoelloosheid en tintelingen in zowel handen als voeten, koude voeten, en moeite met het onderscheiden van kleine objecten in de handen. Patiënten werden door deze klachten beperkt in het dagelijks leven. Problemen met het huishouden werden gemeld in $13 \%$ van de patiënten en $21 \%$ van de patiënten werden meer afhankelijk van anderen vanwege de CIPN symptomen. Bovendien werd de kwaliteit van leven negatief beïnvloed door de CIPN in $49 \%$ van de patiënten.

Door de stijgende leeftijd van de bevolking en de verbeterde behandelingsmogelijkheden voor dikke darmkanker is het aantal patiënten dat dikke darmkanker heeft of heeft gehad toegenomen. De introductie van het geneesmiddel oxaliplatin heeft een grote bijdrage geleverd aan deze verbeterde overleving. CIPN is echter een veel voorkomende bijwerking van oxaliplatin. Vanwege het toenemend aantal patiënten met dikke darmkanker, het toegenomen gebruik van oxaliplatin, en omdat er geen goede behandeling is voor CIPN, worden steeds meer patiënten geconfronteerd met deze bijwerking zowel op de korte als lange termijn. In hoofdstuk 3 onderzochten we het optreden en de ernst van CIPN bij patiënten met dikke darmkanker op de lange termijn. Bovendien onderzochten we de invloed van deze bijwerking op de gezondheid gerelateerde kwaliteit van leven.

De Nederlandse Kankerregistratie, regio Zuidoost Nederland, werd gebruikt om alle nog levende patiënten met dikke darm kanker die in de periode tussen 2000 en 2009 werden gediagnosticeerd te selecteren en 1643 (83\%) van hen namen deel aan het onderzoek. De dikke darmkankerpatiënten rapporteerden twee tot elf jaar na diagnose nog vaak neuropathie symptomen. Met name sensorische symptomen in de onderste ledematen werden vaak vermeld. Patiënten die zijn behandeld met oxaliplatin rapporteerden vaker symptomen als tintelingen (29\% versus $8 \%$ ), 
gevoelloosheid ( $17 \%$ versus $5 \%$ ), en pijn (13\% versus $6 \%$ ) in tenen en/of voeten in vergelijking met patiënten die niet met chemotherapie werden behandeld. Ze rapporteerden ook vaker tintelende tenen of voeten (29\% versus $14 \%$ ) vergeleken met patiënten die zijn behandeld met chemotherapie zonder oxaliplatin. Bovendien rapporteerden patiënten die veel neuropathie symptomen (top 10\%) hadden een klinisch relevante slechtere kwaliteit van leven ten opzichte van patiënten die minder neuropathie symptomen hadden. Samenvattend laat deze studie zien dat neuropathie symptomen twee tot elf jaar na diagnose nog vaak worden ervaren door dikke darmkanker patiënten. Met name patiënten die behandeld zijn met oxaliplatin blijven veel sensorische neuropathie ervaren. De neuropathie symptomen hebben een negatieve invloed op de kwaliteit van leven van deze patiënten.

Een andere populatie patiënten die vaak geconfronteerd worden met de bijwerking CIPN zijn patiënten met multiple myeloom (MM), een vorm van witte bloedcel kanker. Deze patiënten worden behandeld met andere therapie dan de dikke darmkankerpatiënten, en CIPN is voornamelijk het gevolg van behandeling met de chemotherapieën bortezomib en/of thalidomide. Momenteel is er nog geen behandeling voor CIPN en het wijzigen van de chemotherapie dosis of zelfs het staken van de behandeling in geval van matige symptomen van CIPN is de enige manier om ernstige CIPN te voorkomen. Het staken of aanpassen van de chemotherapie dosis kan echter de uitkomst van de behandeling beïnvloeden en moet daarom met de nodige voorzichtigheid worden toegepast. Tot op heden zijn er echter geen hulpmiddelen ontwikkeld die in de dagelijkse praktijk gebruikt kunnen worden om te monitoren of een dosisaanpassing noodzakelijk is. Bovendien is er nog weinig bekend over het optreden van CIPN en de invloed van CIPN op de kwaliteit van leven bij patiënten met MM. In hoofdstuk 4 bestudeerden we enerzijds de validiteit van een vragenlijst die gebruikt kan worden in de dagelijkse praktijk voor het monitoren van CIPN, en anderzijds de prevalentie van CIPN symptomen in patiënten met MM en de invloed van deze symptomen op hun kwaliteit van leven. Honderd-zesenvijftig patiënten (65\% response), gediagnosticeerd met MM in de periode van 2000 tot 2014, vulden gemiddeld 3,3 jaar na diagnose vragenlijsten in. Meer dan de helft van de patiënten rapporteerden neuropathie symptomen en $65 \%$ van de patiënten rapporteerden neuropathie graad 2 of 3 . De nieuw ontwikkelde vragenlijst ICPNQ (Indication Common Toxicity Criteria-grading Peripheral Neuropathy Questionnaire) bleek een waardevol instrument om onderscheid te maken tussen de hoogste en daarmee meest ernstige CIPN graderingen (graad 2 met neuropathische pijn of graad 3 ) en de lagere graderingen (graad 0 tot graad 2 zonder neuropathische pijn). In de dagelijkse klinische praktijk is het van groot belang om een onderscheid te maken tussen deze graderingen, omdat richtlijnen aan de hand van deze graderingen aangeven of een dosisaanpassing nodig is. Op het moment is er echter veel onderrapportage van CIPN door artsen en is het moeilijk voor de artsen om een 
onderscheid te maken tussen deze graderingen. De ICPN is daarom een waardevol instrument dat zou kunnen worden gebruikt in de dagelijkse klinische praktijk.

Verder toonden we in deze studie aan dat de patiënten met de hoogste en daarmee meest ernstige CIPN graderingen (graad 2 met neuropathische pijn of graad 3) een klinisch relevante slechtere kwaliteit van leven hadden dan patiënten met minder ernstige CIPN. Daaruit kunnen we concluderen dat de neuropathie symptomen ook in deze patiëntpopulatie een negatieve invloed hebben op de kwaliteit van leven.

\section{Deel 2- CIPN en de invloed van chemotherapie toediening}

In de literatuur wordt beschreven dat de mate van neuropathie ten gevolge van behandeling met oxaliplatin afhankelijk is van de toegediende totale cumulatieve dosis en de dosis intensiteit. Deze kennis is echter voornamelijk gebaseerd op studies die de ontwikkeling van acute neuropathie tijdens de behandeling beschrijven. De invloed van oxaliplatin toediening op de ontwikkeling van persisterende neuropathie is echter onduidelijk. In hoofdstuk 5 worden de resultaten van een systematische literatuurstudie beschreven. De literatuur met betrekking tot de invloed van oxaliplatin toediening (bijvoorbeeld de totaal ontvangen dosis ofwel cumulatieve dosis, dosis intensiteit, aantal cycli en combinatie regime) op de ontwikkeling van persisterende CIPN ten minste 12 maanden na het beëindiging van behandeling met oxaliplatin werd bestudeerd. Bovendien werd de methodologische kwaliteit van de studies beoordeeld aan de hand van vooraf opgestelde kwaliteitscriteria. Veertien artikelen (totaal 3869 patiënten) die voldeden aan de vooraf gedefinieerde inclusiecriteria werden geïncludeerd. De meerderheid van deze studies was van hoge kwaliteit. De studies toonden dat $\geq 12$ maanden na het beëindigen van de behandeling met oxaliplatin CIPN nog frequent werd ervaren door patiënten. Slechts zes studies hebben de relatie tussen oxaliplatin toediening en de ontwikkeling van persisterende neuropathie onderzocht. Daarvan vonden vijf studies een verband tussen persisterende neuropathie en het toedienen van een hoge totale cumulatieve dosis. Eén studie vond geen relatie tussen persisterende neuropathie en de totale dosis chemotherapie die de patiënt heeft ontvangen. Gezien het feit dat de studies zeer heterogeen waren in de gebruikte hulpmiddelen om CIPN vast te stellen, patiënt populaties en de tijd van CIPN bepaling konden geen duidelijke conclusies getrokken worden met betrekking tot oxaliplatin toediening en het ontwikkelen van CIPN op de lange termijn. Echter, een hogere cumulatieve dosis is waarschijnlijk een voorspellende factor voor de ontwikkeling van CIPN op de lange termijn.

Om deze bevindingen te bevestigen hebben we in hoofdstuk 6 de invloed van oxaliplatin toediening op de ontwikkeling van persisterende CIPN onderzocht in patiënten die dikke darmkanker hebben gehad. Uit de studie van hoofdstuk 3 selecteerden we de patiënten die adjuvant zijn behandeld met oxaliplatin. Vervolgens hebben we uit het medisch dossier van deze patiënten aanvullende gegevens 
verzameld over de behandeling met chemotherapie. Uit de statistische analyses bleek dat het behandelen met een hogere totale dosis oxaliplatin gerelateerd was met het rapporteren van meer persisterende CIPN symptomen. Dosis per tijdseenheid en verlenging van het tijdsinterval tussen de kuren bleek geen invloed te hebben op de mate waarin CIPN werd ervaren op de lange termijn. Tevens bleek dat patiënten die tijdens de chemotherapiekuren een aanpassing van de oxaliplatin dosis hadden gekregen vanwege acuut ontstane neuropathie (50 patiënten) nog steeds meer klachten van CIPN rapporteerden op de lange termijn dan patiënten die geen dosisaanpassing nodig hadden vanwege neuropathie (96 patiënten). Op basis van deze bevindingen hebben we geconcludeerd dat het monitoren van CIPN symptomen tijdens de behandeling zeer belangrijk is, omdat het risico op het ontwikkelen van permanente CIPN enkel kan worden verminderd door het verlagen van de totale dosis oxaliplatin. Daarentegen had het uitstellen van de chemotherapiekuren waarschijnlijk geen invloed op de mate waarin neuropathie werd ervaren op de lange termijn. Daarnaast liepen patiënten die een aanpassing van de oxaliplatin dosis hadden gekregen vanwege acuut opgetreden neuropathie nog steeds het risico om permanente CIPN te ontwikkelen. Daarmee lijkt er een verband te bestaan tussen het optreden van de acute en de chronische vorm van neuropathie ten gevolge van behandeling met oxaliplatin.

De behandeling van $\mathrm{MM}$ is in de laatste decennia zeer sterk verbeterd na de introductie van thalidomide, lenalidomide en bortezomib. Deze behandelingen gaan echter wel gepaard met CIPN. In hoofdstuk 7 hebben we onderzocht wat de invloed is van behandeling met neurotoxische therapie zoals thalidomide, bortezomib, lenalidomide en vincristine op de ontwikkeling van CIPN in patiënten met MM. In totaal hebben 156 patiënten met MM gemiddeld 3,3 jaar na diagnose de neuropathie specifieke vragenlijst, EORTC QLQ-CIPN20, ingevuld met een antwoordpercentage van $65 \%$. Klinische karakteristieken van de patiënten waren beschikbaar via de Nederlandse Kankerregistratie en gedetailleerde behandelingsgegevens werden verkregen via PHAROS. Gegevens over chemotherapie regimes, remissiestatus ten tijde van de studie, en aanpassingen van de doseringen van de therapie werden verzameld. Analyses in deze studie toonden aan dat meer dan de helft van de patiënten met MM tenminste één en gemiddeld drie CIPN symptomen ervaarden die hen behoorlijk of heel veel ergerden in de afgelopen week. Behandeling met thalidomide, en met name een hogere toegediende dosis, bleek van invloed op de ernst van de neuropathie symptomen. De ernst van de CIPN symptomen bleek niet beïnvloed te worden door leeftijd, geslacht, tijd sinds de laatste therapie, aantal ontvangen therapieën, artrose en suikerziekte. Een dosis aanpassing was vaak noodzakelijk (65\%). Patiënten die een dosis aanpassing hadden gekregen door klachten van CIPN rapporteerden desondanks een trend tot ernstigere motorische en sensorische neuropathie vergeleken met patiënten voor wie een dosis aanpassing was 
toegepast met een andere reden of geen dosis aanpassing nodig was. De dosis aanpassingen leken geen invloed te hebben op de uitkomst van de behandeling van patiënten. Daarom lijkt de beslissing tot het aanpassen van de dosis vroeger in de behandeling gerechtvaardigd indien deze is gebaseerd om een betrouwbare manier om CIPN te monitoren. Toekomstig onderzoek moet zich dan ook richten op het gebruik van zelf-gerapporteerde vragenlijsten in de beslissing tot het wijzingen van de dosis, en op het bewerkstelligen van dosis aanpassingen bij minder ernstige CIPN symptomen zonder dat de uitkomst van de behandeling in het geding komt.

Tot slot, worden in hoofdstuk 8 de belangrijkste bevindingen van dit proefschrift samengevat en bediscussieerd. Bovendien worden aanbevelingen gedaan voor toekomstig onderzoek. In dit proefschrift hebben we het optreden en de ernst van CIPN bestudeerd in verschillende patiënt populaties op verschillende tijdstippen na de diagnose kanker. We hebben kunnen concluderen dat CIPN een veel voorkomende bijwerking is van veel gebruikte cytostatica zoals platinum derivaten, taxanen, en middelen gebruikt voor de behandeling van MM. Bovendien heeft CIPN een significante en klinisch relevante negatieve invloed op de kwaliteit van leven van patiënten. In de toekomst dient nog veel onderzoek te worden gedaan naar CIPN. Met name de uitvinding van een hulpmiddel om CIPN te diagnosticeren en monitoren is van groot belang. Indien er een standaard methode is om CIPN vast te stellen kunnen ook betere, zeer noodzakelijke, studies worden gedaan naar zowel de preventie en behandeling, als naar de invloed op kwaliteit van leven. Verder is het van groot belang dat we patiënten kunnen identificeren die een verhoogd risico hebben op het ontwikkelen van CIPN. 
Valorisation 


\section{Introduction}

It is essential for society that academic knowledge is translated into potential societal and economic benefit. This process is called valorisation and is precisely defined as "The process of value creation from knowledge, by making it applicable and available for economic or societal utilization, and by translating it in the form of new business, products, services, or processes" ${ }^{1 .}$ This chapter discusses the potential societal and economic value of the presented results of this thesis.

Cancer is diagnosed in about 100,000 patients each year in the Netherlands. ${ }^{2}$ Systemic treatment like chemotherapy, is an important foundation in the management of many of these cancers. Treatment with cytostatics are accompanied with many adverse events, such as nausea, myelosuppression and peripheral neuropathy, which may have a major impact on patients' quality of life (QOL). In addition, those side effects could be the reason for the clinicians to decide to interrupt, modify or stop the treatment, which may lead to reduced chemotherapy efficacy and survival. Due to improved supportive management options for the adverse events chemotherapyinduced nausea and myelosuppression, chemotherapy-induced peripheral neuropathy (CIPN) has become one of the major dose limiting side effect of commonly used cytostatics.

Because of the increasing prevalence of cancer and the fact that there is still no proven preventive or treatment option for CIPN, more patients are confronted with the consequences of CIPN. Consequently, it is likely that CIPN will become a survivorship issue with major social and economic impact on society. Nevertheless, still many questions about CIPN remain unclear and therefore shedding more light onto the problem of CIPN and making patients and physicians more aware of this vital adverse event is of major importance for society. The results of this thesis have contributed to insight in many of those unanswered questions (Table 10.1).

\section{Part I - Chemotherapy-induced peripheral neuropathy and quality of life}

Until now the extent of CIPN remained unclear for society as the literature about the clinical manifestation and incidence of CIPN was very diverse due to the heterogeneity of studies and the use of different assessment methods. In addition, symptoms were most often reported by clinicians who are known to underreport CIPN and studies mainly focused on the short-term symptoms during treatment while the long-term consequences of reported symptoms and their impact on society remained unknown. The results in this thesis aid in increasing the knowledge about CIPN as we have 
investigated which particular symptoms are most reported by different patient populations according to patient-reported outcomes, and which symptoms are most debilitating for them at the short- and long-term.

The included population-based studies confirmed that CIPN is a severe, unpredictable, and often irreversible adverse event of frequently used cytostatics, such as taxanes, oxaliplatin and antimyeloma treatment such as thalidomide and bortezomib. Patients reported mainly symptoms of tingling, numbness, pain and loss of strength in the hands and/or legs (chapter 2, 3, 4, 7). Those symptoms resulted in problems in e.g. fine motor skills, such as managing the computer, difficulty in writing or managing money, or problems in opening a bottle or climbing stairs due to loss of strength in hands or legs. We also clarified that many CRC survivors and patients with MM keep being confronted with the consequences of the neuropathy symptoms more than 10 years after diagnosis (chapter 3, 4, 7).

Before our research little was known about the influence of these symptoms on patients' functioning and QOL. We made clear that those symptoms not only influence their daily activities, but also have a major impact on their overall QOL (chapter 2, 3, 4). Patients who experienced the most severe symptoms of CIPN reported statistically and clinically relevant worse scores on all the domains of QOL including their physical, emotional and social functioning. With these findings we have elucidated that the social impact of CIPN is larger than expected and that the importance of awareness of this potentially severe side effect is vital for both patients and physicians.

Unfortunately, little is still known about the potential economic impact of the CIPN symptoms. Up to $30 \%$ of CRC survivors treated with oxaliplatin reported to experience severe CIPN symptoms two to 11 years after diagnosis (chapter 3 ) and more than half of patients with MM reported severe CIPN up to 13 years after diagnosis (chapter 4, 7). These patients are long-term cancer survivors and supposed to be reintegrated in society. However, it could be hypothesized that those patients are not able to work or economically contribute to society because of those symptoms. Moreover, guidelines recommend treatment with duloxetine, gabapentin, or pregabalin for chemotherapyinduced neuropathic pain. ${ }^{3,4}$ Evidence concerning the effectivity of those drugs in case of neuropathic pain due to CIPN is however scarce. ${ }^{3}$ Nonetheless, many patients are using these drugs in spite of lack of proven effectiveness which results in substantial costs for society. To my knowledge only one study has been performed which investigated the influence of CIPN on patients' workability ${ }^{5}$, and none concerning healthcare usage and the resulting potential economic impact. Therefore, as our research indicates that long-term CIPN is more often reported than expected, a likely next step in research is to evaluate the potential economic impact of CIPN by evaluating healthcare usage or work disability because of CIPN. 


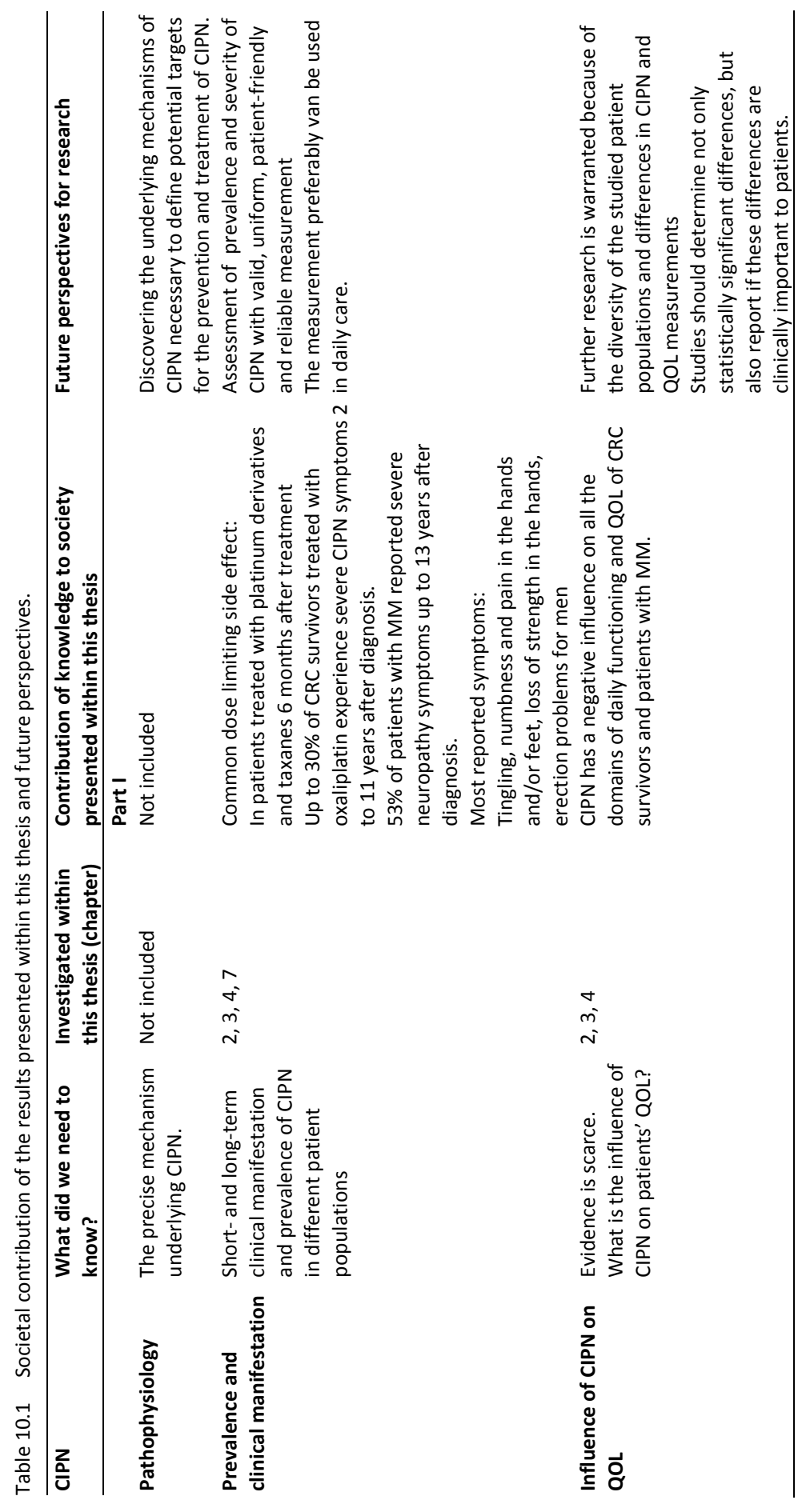




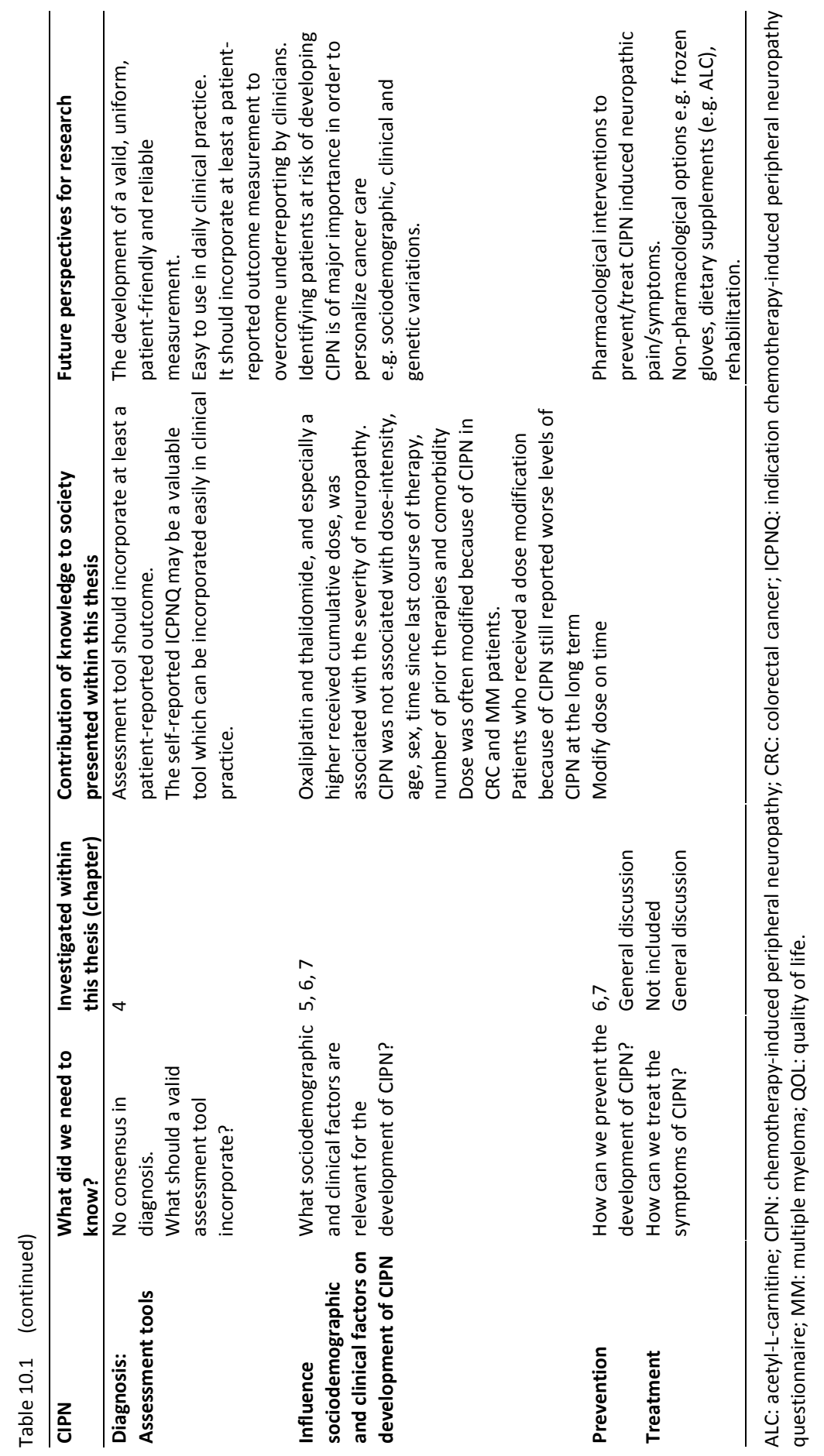


Another issue, which has been debated and impeded the knowledge of the clinical manifestation of CIPN and its influence on QOL before, is the lack of a consensus on diagnostic assessment tools to measure CIPN. ${ }^{6}$ Since clinicians tend to underestimate CIPN by using the common toxicity grading scales and objective measurements are not patient-friendly, expensive and do not correlate with subjective symptoms, the use of a self-reported questionnaire has been recommended. ${ }^{3}$ In our studies, we therefore used self-reported questionnaires to evaluate CIPN. However, although highly needed, it should be mentioned that most assessment tools including questionnaires are not developed nor used in daily clinical practice at the moment. Therefore, in chapter 4 we also validated a self-reported measure (ICPNQ) which has been made to be applicable for daily clinical practice. The ICPNQ appeared to be a valid instrument to distinguish the higher grades of CIPN from the lower grades in a uniform way and overcomes the issue of underreporting CIPN by clinicians.

This questionnaire can be used in daily clinical practice since it is based on the common toxicity criteria which are used in current guidelines to decide on dose modifications. Further prospective evaluation of this questionnaire however is needed in order to evaluate the ability to monitor the development of CIPN over time, and to compare the self-reported neuropathy with physician reported neuropathy, and its use in decisions regarding dose modifications.

\section{Part II - CIPN and the influence of chemotherapy administration}

Currently, dose modification schemes are the only way to prevent moderate CIPN symptoms to become more severe. As CIPN potentially has a major societal and economic impact, it is of major importance for society to be able to identify patients at risk of developing CIPN on time, or preferably before the start of treatment. Accordingly, patients who are prone to develop CIPN can benefit from personalized medicine when deciding on the best therapy with the least toxicity. At the moment, patients with preexisting neuropathy who are more likely to develop CIPN are identified before treatment, but otherwise it is difficult to completely recognize patients who are prone to develop CIPN. There are signs that encoding genes are involved in the development of CIPN. However, since genomic studies are still in its infancy and not routinely used in usual care, for the time being it is important to identify sociodemographic and clinical characteristics involved in the development of CIPN. Within part II of this thesis, we investigated the influence of chemotherapy administration and dose modifications on the development of CIPN (chapter 5, 6, 7). These studies contribute to the knowledge about the development of persistent CIPN. Within these three chapters we reported that the dose was often modified because of 
CIPN and that especially a higher received cumulative dose of oxaliplatin in CRC patients and thalidomide in patients with MM was an important risk factor for the development of long-term CIPN (chapter 5, 6, 7). CIPN was not associated with doseintensity, age, sex, time since last course of therapy, number of prior therapies and comorbidity. The societal value lies in the fact that this emphasized that monitoring of CIPN during treatment is of major importance as dose might be modified on time. To do so, as mentioned earlier, a proper assessment tool which can be used in daily care is essential. The questionnaire presented in chapter 4 might be a valuable tool for this purpose.

Furthermore, cancer response rates were described to be the same between patients who received or did not receive a dose modification because of CIPN (chapter 6,7). Nonetheless, study design and sample sizes of our studies were not intended to investigate this question. However, it would be of great interest to investigate if dose modifications could be safely applied at lower levels of CIPN without compromising response rates. If this is possible, patients will experience lower levels of CIPN at the long-term with societal benefit. In addition, also economically this would be of value as the costs of chemotherapy and symptom-alleviating drugs could be lowered. Moreover, as patients have lower levels of CIPN they might be able to live longer independently without extra care.

In conclusion, the main value for society of the presented studies in this thesis are numerous as still many questions about this common, frequently dose limiting and debilitating side effect are unanswered. This thesis sheds more light on the problem of CIPN and emphasizes that CIPN has a major societal impact as many patients suffer from difficulties in daily activities and have a worse QOL because of CIPN. Furthermore, CIPN has a major hypothesized economic impact on the society which should be further investigated. As some patients are likely to be more prone to develop CIPN, personalized medicine should be more integrated to prevent patients and society from the burden of this devastating side effect. For the near future especially the usage of a valid assessment tool to measure CIPN in a reliable way is important. This is not only important for proper monitoring CIPN during treatment, but also highly needed for suitable research concerning al the aspects of CIPN which are still unanswered. 


\section{References}

1. Regulations governing the attainment of doctoral degrees. Maastricht University 2013:51.

2. http://www.cijfersoverkanker.nl/incidentie-sterfte-50.html (Accessed on 15 July, 2016).

3. Hershman DL, Lacchetti C, Dworkin RH, Lavoie Smith EM, Bleeker J, Cavaletti G et al. Prevention and Management of Chemotherapy-Induced Peripheral Neuropathy in Survivors of Adult Cancers: American Society of Clinical Oncology Clinical Practice Guideline. J Clin Oncol 2014;32:1941-1967.

4. van den Beuken-van Everdingen MH, de Graeff A, Jongen JL, Dijkstra D, Mostovaya I, Vissers KC, et al. Pharmacological Treatment of Pain in Cancer Patients: The Role of Adjuvant Analgesics, a Systematic Review. Pain Pract. 2016.

5. Zanville NR, Nudelman KN, Smith DJ, Von Ah D, McDonald BC, Champion VL, et al. Evaluating the impact of chemotherapy-induced peripheral neuropathy symptoms (CIPN-sx) on perceived ability to work in breast cancer survivors during the first year post-treatment. Support Care Cancer. 2016 Jul 28.

6. Cavaletti G, Frigeni B, Lanzani F, Mattavelli L, Susani E, Alberti P, et al. Chemotherapy-Induced Peripheral Neurotoxicity assessment: a critical revision of the currently available tools. Eur J Cancer. 2010 Feb;46(3):479-494. 
Dankwoord 


\section{Dankwoord}

Afgelopen jaren heb ik met veel plezier en inzet gewerkt aan de totstandkoming van dit proefschrift. Het was niet altijd makkelijk en ik had natuurlijk zo mijn ups-anddowns, maar met behulp van vele mensen ben ik deze te boven gekomen en heb dit mooie resultaat bereikt. Ik ben dan ook veel mensen dankbaar die hebben bijgedragen aan de realisatie van dit proefschrift, want zonder hun hulp had ik dit resultaat nooit bereikt.

Allereerst wil ik de patiënten die hebben deelgenomen aan de studies van harte bedanken. Ik heb grote bewondering voor hen die gedurende de zware periode van hun ziekte toch willen bijdragen aan wetenschappelijk onderzoek om zo patiënten in de toekomst te helpen. Hartelijk dank!

Verder wil ik natuurlijk mijn promotieteam van harte bedanken.

Dr. Vreugdenhil, beste Art, zonder jou had ik dit resultaat nooit behaald. Ik ben als semi-arts onder begeleiding van jou begonnen aan mijn onderzoeksstage en deze stage is uitgemond in een mooie publicatie. Om tot deze publicatie te komen moest ik tijdens mijn reis door China een major revision indienen met goed resultaat. Dit wekte bij jou het vertrouwen om samen met mij de onderzoekslijn naar CIPN uit te bereiden en met behulp van het overige promotieteam hebben we het onderzoek zelfs kunnen omvormen tot een volwaardig promotietraject. Ondanks de drukte van de praktijk als AIOS heeft jouw enthousiasme er mede voor gezorgd dat ik het onderzoek met veel plezier deed. Jouw kennis, innovatieve ideeën en aandacht voor de onderliggende boodschap van de artikelen hebben een grote bijdrage geleverd aan dit proefschrift. Ik hoop dat we ook in de toekomst nog meer onderzoek samen kunnen doen. Verder hoop ik in de toekomst nog veel van je leren in de medische praktijk van het ziekenhuis.

Dr. Mols, beste Floor, ook zonder jou had ik dit resultaat nooit bereikt. Hartelijke dank voor je altijd zeer snelle en kritische commentaar. Ik heb mede door jouw inzet in een korte tijd veel kunnen bereiken. Ik waardeer dit heel erg. Tevens wil ik je bedanken voor je aanstekelijke enthousiasme voor het onderzoek. Jij hebt me laten zien hoe leuk onderzoek doen is en me de fijne kneepjes van het vak geleerd; hoe zet ik een onderzoek op, hoe analyseer ik de data en hoe schrijf ik een goed artikel. Ik hoop dat we in de toekomst nog veel onderzoek samen zullen doen en de kennis mbt. CIPN kunnen vergroten. 
Prof. Dr van de Poll-Franse, beste Lonneke, je bood mij de kans om mijn onderzoek bij het IKNL uit te bereiden. Verder vond je ondanks je drukke schema, altijd de tijd om mijn manuscripten te voorzien van zeer waardevolle (epidemiologische) aanvullingen. Hartelijk dank.

Prof. Dr. Tjan-Heijnen, beste Vivianne, hartelijk dank voor het vertrouwen in mijn onderzoek en de snelle en soms confronterende, maar zeer waardevolle aanvullingen die ik kon ontvangen op mijn stukken.

De leden van de beoordelingscommissie, prof. dr. Koopmans, dr. Lalisang, prof. dr. Schouten, dr. Jongen en prof. dr. van der Rijt, wil ik hartelijk danken voor de moeite en tijd die jullie hebben besteed aan het grondig lezen en beoordelen van mijn proefschrift.

Overige medeauteurs, dr. Dercksen, Chantal Driessen, prof. dr. Lemmens, Corina van den Hurk, Simone Oerlemans, dr. Eurelings, dr. Minnema, Corien Eeltink en prof. dr. Faber, hartelijk dank voor jullie tijd, medewerking en kritische commentaar.

Paranimfen Hanneke en Steffie. Hanneke, grote zus, hartelijk dank voor je steun en advies de afgelopen jaren. Jij weet als geen ander hoe zwaar een promotietraject soms kan zijn en hebt mij hier dan ook goed in kunnen ondersteunen. Jij hebt afgelopen jaar je opleiding tot internist al afgerond en een mooie baan bemachtigd in Boxmeer als internist-endocrinoloog. Ik hoop dat ik me over een paar jaar collegainternist kan noemen!

Steffie wij hebben een vergelijkbaar pad belopen in het MMC. Samen gestart als semiarts en ons onderzoek uitgebreid tot een promotietraject. Het onderzoek hebben we beide gecombineerd met het werk als escalatiearts en nu zijn we beide AIOS interne geneeskunde in het MMC. Hier bovenop hebben we ook allebei afgelopen jaar een prachtige zoon gekregen. Het onderzoek en de combinatie met deze andere bezigheden is en was niet altijd makkelijk en soms heel frustrerend. Een keer bij elkaar klagen en grappen is dan ook heel fijn $:-$. Dank dat je zo'n fijne collega bent en ik heb er vertrouwen in dat jouw proefschrift ook snel tot een goed resultaat komt.

Mede-onderzoekers van de interne geneeskunde en escalatie collega's Steffie, Hester, Roosmarijn, Dorien, Joyce en Thomas, ik wil jullie hartelijk danken voor de gezelligheid, jullie flexibiliteit bij de escalatie en luisterende oor als ik/we het even niet meer zagen zitten. Helaas kon ik niet elke dag bij jullie in MMC-Eindhoven zitten, maar ik was in overleg natuurlijk altijd welkom indien er wel plek was. Onderzoek doen in het perifere MMC is, zoals we allen hebben ervaren, niet altijd makkelijk. Toch slagen we er allen in om een mooie onderzoekslijn op te zetten en ik kijk uit naar jullie ongetwijfeld mooie proefschriften. 
Internisten en MDL-artsen van het Máxima Medisch Centrum, ik wil jullie danken voor jullie interesse in mijn onderzoek en de altijd prettige samenwerking en opleidingscultuur. Ik leer heel veel van jullie en hoop in de toekomst nog veel meer van jullie te leren.

Collega (ex-)A(N)IOS van de interne geneeskunde dank jullie wel voor de prettige samenwerking, flexibiliteit en gezelligheid binnen de groep. Ik ben zeer dankbaar voor de prettige atmosfeer in het MMC waarin wij samen ons werk doen. Zonder jullie had ik nooit het doorzettingsvermogen gehad om het onderzoek te blijven combineren naast mijn werk in het ziekenhuis.

Verpleegkundig specialisten, en in het bijzonder Joyce Ophorst, en verpleging van de dagbehandeling, hartelijk dank voor jullie hulp bij de Frozen glove studie en interesse in mijn studies. Jullie verrichten heel mooi en belangrijk werk en hopelijk kunnen we nog lang samenwerken.

Overige leden van het escalatieteam bedankt voor jullie interesse en prettige samenwerking in het MMC-Eindhoven. Ik heb zeer dankbaar dat ik de mogelijkheid heb gekregen om in de avond/nacht bij het escalatieteam te werken zodat ik overdag aan mijn onderzoek kon werken. Ik heb een leuke tijd gehad bij de escalatie.

Collega's van het IKNL en datamanagers hartelijk dank voor jullie gastvrije en gezellige ontvangst bij het IKNL en jullie hulp bij mijn studies. Jullie hebben echt een heel leuk team en ik voelde me meteen welkom bij jullie. Mede dankzij jullie kijk ik met heel veel plezier terug op mijn promotietraject. Bedankt!

Mijn vriendinnengroep, Mirjam, Loes, Aukelien, Marianne, Lia, Irma, Jenny, Andrea. Hartelijk dank voor de gezellige avonden, etentjes en dagjes uit. Ik ben heel dankbaar dat wij na al die jaren nog zo'n goede vriendinnen zijn. Jammer genoeg zijn we het afgelopen jaar iets te dicht geconfronteerd met een van de vreselijke ziektes waar mijn proefschrift over gaat. Lia, ik heb hele grote bewondering voor de manier waarop jij met je ziekte omgaat en voor je doorzettingsvermogen! Je hebt mij doen inzien dat het leven niet altijd loopt zoals je het plant, en dat je echt nu moet genieten van het leven en de kleine dingen. Gelukkig heb je alles tot dusver goed doorstaan en gaat het nu naar omstandigheden weer goed met je. Dikke knuffel.

Mijn schoonfamilie, Wim, Wies, Bart, Eva, Rink, Hans en Suze. Ik ben echt heel blij dat ik jullie mijn familie mag noemen. Ik heb me altijd meer dan welkom gevoeld. Hans bedankt voor het maken van de kaft. Wim en Wies heel erg bedankt voor alles wat jullie voor ons doen. Jullie staan altijd voor ons klaar en mede dankzij jullie oneindige 
wil om ons te helpen in en rondom ons huis heb ik de tijd gevonden om mijn proefschrift af te ronden.

Pap en mam bedankt voor jullie oneindige vertrouwen in ons. Jullie hebben altijd alles opzij gezet om ons de mogelijkheid te bieden om ons te ontwikkelen en te gaan studeren. Ik hou van jullie!

Mijn zussen en broer, Fien, Hanneke en Bert, en schoonbroers/zus Martijn, Marten en Amy en nichtje Janne. Ik ben heel trots op onze familie en het feit dat we het zo goed met elkaar kunnen vinden en dat we altijd bij elkaar terecht kunnen.

Tot slot wil ik bedanken mijn allerliefste man Rudy. Lieve Rudy hartelijk dank voor je steun en vele relativerende woorden tijdens de niet altijd makkelijke momenten gedurende dit lange traject. Jij zorgde ervoor dat ik, ondanks mijn drukke schema, ook mijn ontspanning pakte en de computer eens uit durfde te zetten. Zonder deze momenten en de stabiele basis bij jou had ik het nooit tot een goed resultaat kunnen brengen. Het proefschrift was ook precies op tijd af, want de dag waarop ik het heb ingeleverd hebben we het allermooiste geschenk gekregen dat we ons konden wensen, onze zoon Jens. Ik hou van jullie! 
About the author 


\section{About the author}

Tonneke (Antoinetta Josephina Maria) Beijers was born on the $4^{\text {th }}$ of February 1987 in Nijmegen, the Netherlands. She grew up in a small town called Asten. She finished secondary education at the Varendonck College in Asten in 2005 and subsequently started medical school at Maastricht University. In 2008 she received her Bachelor's degree in Medicine. Subsequently, she started her regular internships, and conducted her scientific internship at the Máxima Medical Center in the last year of the master program. This internship focused on the prevention of chemotherapy-induced peripheral neuropathy and was conducted under supervision of Dr. Vreugdenhil (Hematologist-Oncologist, Máxima Medical Center). After obtaining her master degree in medicine in August 2011 she traveled for 4 months in Asia and Australia. Afterwards in the beginning of 2012, she started working as a resident (ANIOS) at the internal medicine department of the Máxima Medical Center in Veldhoven. Simultaneously during this year she expanded her research focusing on chemotherapy-induced peripheral neuropathy under supervision of Dr. Vreugdenhil and Dr. Mols, assistant professor in medical psychology at Tilburg University. In the beginning of 2013 the research was extended to a PhD program under supervision of prof. dr. V.C.G. Tjan-Heijnen (Medical Oncology, Maastricht University Medical Centre+) and prof. dr. L.V. van de Poll-Franse (Research, Netherlands Comprehensive Cancer Organisation and Tilburg University). Her research focused on the prevalence of chemotherapy-induced peripheral neuropathy, its impact on patients' quality of life, and the influence of chemotherapy administration on the development of chemotherapy-induced peripheral neuropathy. From 2013 to 2016 the PhD program was combined with working as a general hospital doctor in the Máxima Medical Center. She currently works as a resident in internal medicine at the Máxima Medical Center in Eindhoven/Veldhoven (head: dr. A.G. Lieverse) as part of her specialty training at Maastricht University Medical Centre+ (head: prof. dr. C.D.A. Stehouwer). 
List of publications 


\section{List of publications}

F. van Erning, M. Janssen-Heijnen, J. Wegdam, G. Slooter, J. Wijsman, A Vreugdenhil, T. Beijers, L. van de Poll-Franse, V. Lemmens. The course of neuropathic symptoms in relation to adjuvant chemotherapy among elderly stage III colon cancer patients: a longitudinal study. Clin Colorectal Cancer. 2016 Sep.

A. Beijers, S. Oerlemans, F. Mols, M. Eurelings, M.C. Minnema, G. Vreugdenhil, L.V. van de Poll Franse. The magnitude of neurotoxicity in patients with multiple myeloma and the impact of dose modifications: Results from the population-based PROFILES study. Submitted Ann Hematol. Major Revision.

D. van Dam, A. Beijers, G. Vreugdenhil. Acetyl-L-Carnitine undervalued in the treatment of Chemotherapy-Induced Peripheral Neuropathy? Acta Oncol. 2016 Sep 8:1-3

F. Mols, L. van de Poll-Franse, G. Vreugdenhil, A. Beijers, J. Kieffer, N. Aaronson, O. Husson. Reference data of the EORTC QLQ-CIPN20 questionnaire in the general Dutch population. Eur J Cancer. 2016. In press.

A. Beijers, G. Vreugdenhil, S. Oerlemans, M. Eurelings, M.C. Minnema, C. Eeltink, L.V. van de Poll-Franse, F. Mols. Chemotherapy-induced neuropathy in multiple myeloma: influence on quality of life and development of a questionnaire to compose Common Toxicity Criteria-grading for use in daily clinical practice. Support Care Cancer. 2016 Jun;24(6):2411-20.

A. Beijers, F. Mols, C.J. van den Hurk, G. Vreugdenhil. Are chemotherapy-associated symptoms underestimated? A view beyond Common Toxicity Criteria. Acta Oncol. 2016 Apr;55(4):516-8.

F. Mols, A. Beijers, G. Vreugdenhil, A. Verhulst, G. Schep, O. Husson. Chemotherapyinduced peripheral neuropathy, physical activity and health-related quality of life among colorectal cancer survivors from the PROFILES registry. J Cancer Surviv. 2015 Sep;9(3):512-22.

A. Beijers, A.L.J. Verhulst, F. Mols, G. Vreugdenhil. Comment on: 'Subgroup effects in a randomized trial of different types and doses of exercise during breast cancer chemotherapy'. Br J Cancer. 2015 May 26;112(11):1835. 
A. Beijers, F. Mols, V.C.G. Tjan-Heijnen, C. Faber, L.V. van de Poll-Franse, G. Vreugdenhil. Peripheral neuropathy in colorectal cancer survivors: The influence of oxaliplatin administration. Results from the population-based PROFILES registry. Acta Oncol. 2015 Apr;54(4):463-9.

A. Scheel, A. Beijers, F. Mols, C.G. Faber, G. Vreugdenhil. Chemotherapiegeïnduceerde perifere neuropathie en invloed op kwaliteit van leven. Ned Tijdschr Geneeskd. 2014;158:A7455.

F. Mols, T. Beijers, G. Vreugdenhil, L.V. van de Poll-Franse. Chemotherapy-induced peripheral neuropathy and its influence on quality of life: A systematic review. Support Care Cancer. 2014 Aug;22(8):2261-9.

A. Beijers, F. Mols, C.M.L. Driessen, M. Dercksen, G. Vreugdenhil. Chemotherapyinduced peripheral neuropathy and impact on quality of life six months after treatment with taxanes and platinum derivatives. J Community Support Oncol. 2014 Nov;12(11):401-6.

A. Beijers, F. Mols, G. Vreugdenhil. A systematic review on chronic oxaliplatin-induced peripheral neuropathy and the relation with oxaliplatin administration. Support Care Cancer. 2014 Jul;22(7):1999-2007.

F. Mols, T. Beijers, V. Lemmens, C. Hurk, G. Vreugdenhil, L.V. van de Poll-Franse. Chemotherapy-induced neuropathy and its association with quality of life among 2-11 year colorectal cancer survivors; results from the population-based PROFILES registry. Journal of Clinical Oncology. 2013 Jul ;31(21):2699-707.

A. Beijers, J.L.M. Jongen, G. Vreugdenhil; Chemotherapy-induced neurotoxicity: the value of neuroprotective strategies. Neth J Med. 2012 Jan;70(1):18-25.

Ophorst J, T. Beijers, L. Derksen, S. Houterman, G. Vreugdenhil. Handkoeling ter preventie van chemotherapie geïnduceerde perifere neuropathie: een pilotstudie en overzicht van neuroprotectieve middelen. Oncologica. 2012; 2.

\section{Abstract presentations}

Adjuvant oxaliplatin dose and dose reductions are associated with severity of neuropathy symptoms among colorectal cancer survivors; results from the population-based PROFILES registry.

Poster Display presentation ESMO conference Madrid, 25 till 30 September 2014. 
Neuropathie bij patiënten met Multiple Myeloom (MM) en de invloed ervan op kwaliteit van leven.

Oral presentation, Symposium Netherlands Cancer Registry, Utrecht, February 2015.

Chemotherapy-induced peripheral neuropathy in multiple myeloma patients. Results from the PROFILES registry.

Oral presentation, Congress of the Dutch society of internal medicine, Maastricht, 22 till 24 April 2015.

Chemotherapy-induced neuropathy among Multiple Myeloma patients and the influence of chemotherapeutic agents: Results from the population-based PROFILES Registry.

Poster Display presentation European Hematology Association, Vienna, 11 till 14 June 2015.

Chemotherapy-induced peripheral neuropathy in multiple myeloma: influence on quality of life and validation of a questionnaire to compose Common Toxicity Criteriagrading for use in daily clinical practice.

Oral presentation, Multinational Association of Supportive Care in Cancer Annual meeting, Copenhagen, 25 till 27 of June 2015.

Pijn / CIPN: Chemotherapie geïnduceerde perifere neuropathie.

Oral presentation, Nederlandse Vereniging voor Fysiotherapie binnen de Lymfologie, Harderwijk, April 2016. 
